Dinitrogen fixation, partial reduction and formation of coordinated imide promoted by a chromium bis-iminopyridine complex.

Indu Vidyaratne, Jennifer Scott, Sandro Gambarotta,* Peter H.M. Budzelaar*

Department of Chemistry, University of Ottawa, Ottawa, Ontario K1N 6N5, Canada

Department of Chemistry, University of Manitoba, Winnipeg, Man. R3T 2N2 (Canada) 
IR Spectrum of complex 7- $\left({ }^{14} \mathrm{~N}\right)$

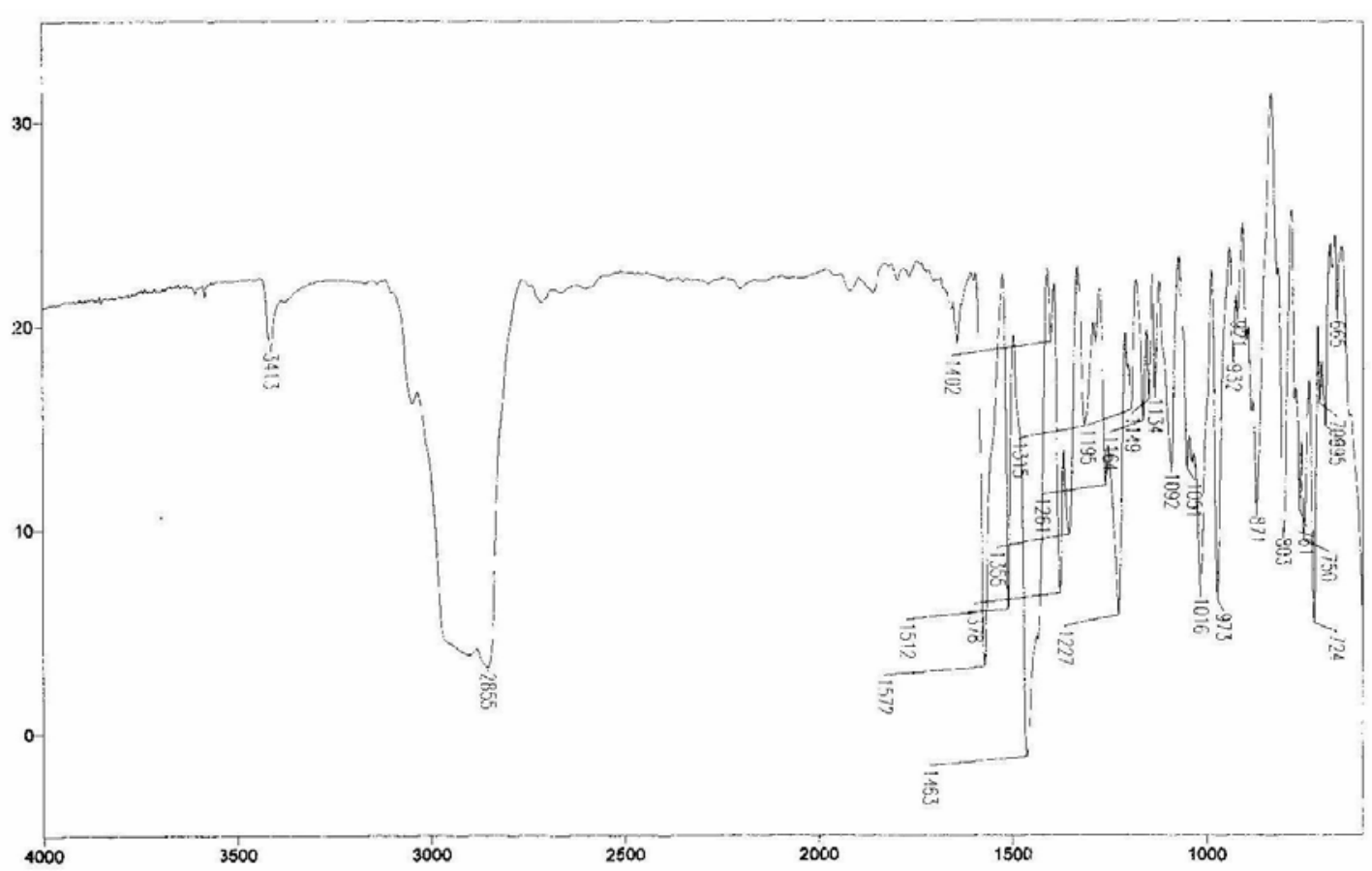

Transmissicn / Waventmber ( $\mathrm{cm}-1)$

Paged $Y$-Zoorn CURSOF

File \# 1 : CRBH(TOL)N14

8/16/2006 9:40 AM Rek=4

IR Spectrum of complex 7- $\left({ }^{15} \mathrm{~N}\right)$

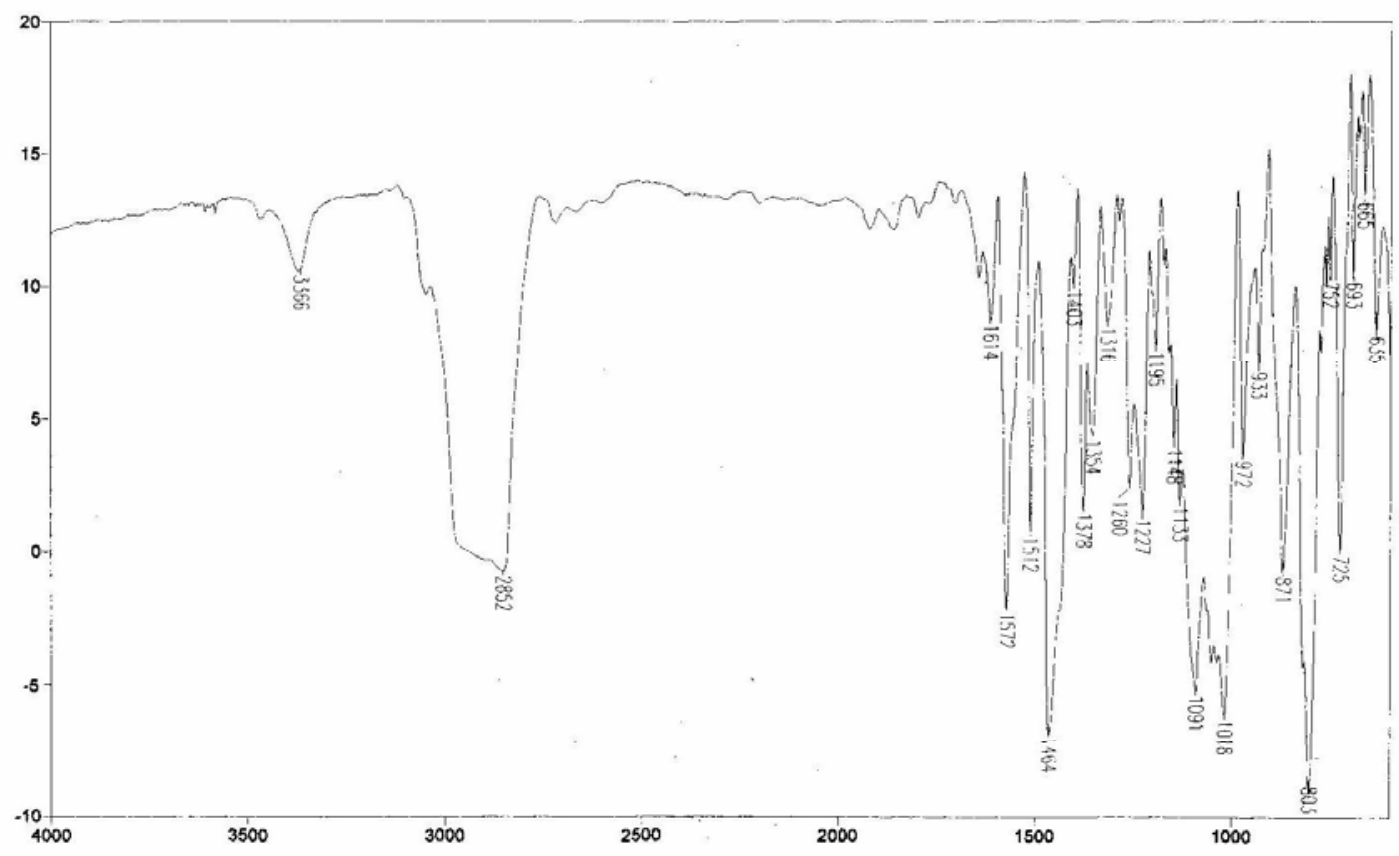

Transmission / Wavenumber (cm-1)

File \# 1 : CRBH(TOL)N15-2

Paged Y-Zoom CURSDF 8/16/2006 10:42 AM Reb=4 


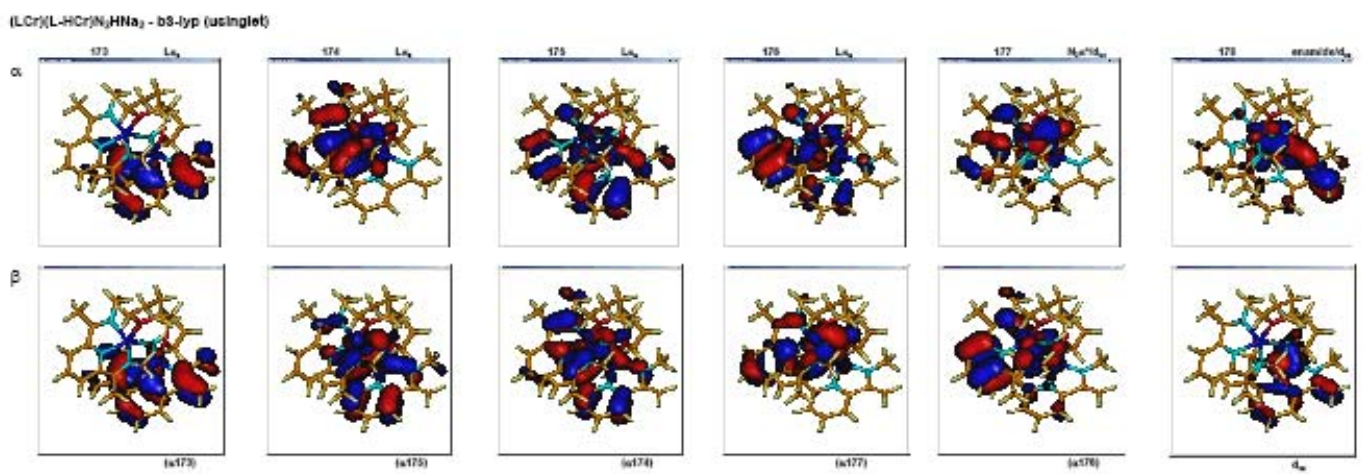

To open the above .pdf file please double click on the picture 
Complex 4 
Table S1. Crystal data and structure refinement for 4 .

\begin{tabular}{|c|c|}
\hline Identification code & sg3023a \\
\hline Empirical formula & C37 H50 Cr N3 O \\
\hline Formula weight & 604.80 \\
\hline Temperature & $200(2) \mathrm{K}$ \\
\hline Wavelength & $0.71073 \mathrm{~A}$ \\
\hline Crystal system, space group & Orthorhombic, $\quad$ P2(1)2(1)2(1) \\
\hline Unit cell dimensions & $\begin{array}{l}a=20.730(4) A \quad \text { alpha }=90 \text { deg. } \\
b=8.6418(16) A \quad \text { beta }=90 \mathrm{deg} . \\
c=19.063(4) A \quad \text { gamma }=90 \mathrm{deg} .\end{array}$ \\
\hline Volume & $3415 . \odot(11) A^{\wedge} 3$ \\
\hline Z, Calculated density & 4, $1.176 \mathrm{Mg} / \mathrm{m} \wedge 3$ \\
\hline Absorption coefficient & $0.366 \mathrm{~mm}^{\wedge}-1$ \\
\hline$F(\odot \odot \odot)$ & 1300 \\
\hline Crystal size & $0.20 \times 0.15 \times 0.10 \mathrm{~mm}$ \\
\hline Theta range for data collection & 1.45 to $23.26 \mathrm{deg}$ \\
\hline Limiting indices & $-23<=h<=23, \quad-9<=k<=9, \quad-21<=1<=21$ \\
\hline Reflections collected / unique & $22260 / 4842[R($ int $)=0.0338]$ \\
\hline Completeness to theta $=23.26$ & $97.9 \%$ \\
\hline Absorption correction & Semi-empirical from equivalents \\
\hline Max. and min. transmission & $\odot .9643$ and $\odot .9303$ \\
\hline Refinement method & Full-matrix least-squares on $F^{\wedge} 2$ \\
\hline Data / restraints / parameters & $4842 / \odot / 379$ \\
\hline Goodness-of-fit on $F^{\wedge} 2$ & 1.049 \\
\hline Final $R$ indices $[I>2 \operatorname{sigma}(I)]$ & $R 1=0.0425, W R 2=0.1102$ \\
\hline $\mathrm{R}$ indices (all data) & $R 1=0.0499, \quad W R 2=0.1159$ \\
\hline Absolute structure parameter & $\odot . \odot \odot(2)$ \\
\hline Largest diff. peak and hole & 0.381 and -0.198 e. $A^{\wedge}-3$ \\
\hline
\end{tabular}


Table S2. Atomic coordinates $\left(\times 10^{\wedge} 4\right)$ and equivalent isotropic displacement parameters $\left(A^{\wedge} 2 \times 10 \wedge 3\right)$ for 4. $\mathrm{U}(\mathrm{eq})$ is defined as one third of the trace of the orthogonalized Uij tensor.

\begin{tabular}{|c|c|c|c|c|}
\hline & $x$ & $y$ & z & $\mathrm{U}(\mathrm{eq})$ \\
\hline $\operatorname{cr}(1)$ & $1349(1)$ & $6046(1)$ & $8988(1)$ & $37(1)$ \\
\hline$N(1)$ & $549(1)$ & $6176(3)$ & $9591(1)$ & $39(1)$ \\
\hline$N(2)$ & $752(1)$ & $4774(3)$ & $8451(1)$ & $36(1)$ \\
\hline$N(3)$ & $1904(1)$ & $5455(3)$ & $8160(2)$ & $39(1)$ \\
\hline $0(1)$ & $1997(1)$ & $7356(3)$ & $9575(1)$ & $56(1)$ \\
\hline$C(1)$ & $496(2)$ & $7076(4)$ & $10225(2)$ & $41(1)$ \\
\hline$C(2)$ & $485(2)$ & $8692(4)$ & $10174(2)$ & $54(1)$ \\
\hline$C(3)$ & $503(2)$ & $9557(5)$ & $10790(2)$ & $65(1)$ \\
\hline$C(4)$ & $542(2)$ & $8874(6)$ & $11431(2)$ & $67(1)$ \\
\hline$C(5)$ & $544(2)$ & $7259(5)$ & $11479(2)$ & $58(1)$ \\
\hline$C(6)$ & $516(2)$ & $6352(4)$ & $10882(2)$ & $43(1)$ \\
\hline$C(7)$ & $788(3)$ & $11017(6)$ & $9416(3)$ & $9 \odot(2)$ \\
\hline$C(8)$ & $431(2)$ & $9504(5)$ & $9467(2)$ & $68(1)$ \\
\hline$C(9)$ & $-270(4)$ & $9782(8)$ & $9277(3)$ & $115(2)$ \\
\hline$c(10)$ & $7 \odot(2)$ & $3998(6)$ & $11512(3)$ & $78(1)$ \\
\hline$c(11)$ & $527(2)$ & $4587(4)$ & $10950(2)$ & $48(1)$ \\
\hline$C(12)$ & $1206(2)$ & $3982(5)$ & $11068(3)$ & $72(1)$ \\
\hline$c(13)$ & $-603(2)$ & $5421(5)$ & $9661(2)$ & $62(1)$ \\
\hline
\end{tabular}




\begin{tabular}{|c|c|c|c|c|}
\hline$c(14)$ & $17(2)$ & $5405(4)$ & $9340(2)$ & $43(1)$ \\
\hline$C(15)$ & $142(2)$ & $4581(4)$ & $8698(2)$ & $39(1)$ \\
\hline$C(16)$ & $-295(2)$ & $3637(4)$ & $8326(2)$ & $49(1)$ \\
\hline$c(17)$ & $-80(2)$ & $2929(5)$ & 7705 (2) & $63(1)$ \\
\hline$C(18)$ & $540(2)$ & $3130(4)$ & $7465(2)$ & $50(1)$ \\
\hline$c(19)$ & $962(2)$ & $4093(4)$ & $7836(2)$ & $39(1)$ \\
\hline$c(20)$ & $1609(2)$ & $4510(4)$ & $7670(2)$ & $42(1)$ \\
\hline$C(21)$ & $1923(2)$ & $3995(5)$ & $7034(2)$ & $55(1)$ \\
\hline$C(22)$ & $2538(2)$ & $6039(4)$ & $8038(2)$ & $38(1)$ \\
\hline$C(23)$ & $3065(2)$ & $5366(5)$ & $8379(2)$ & $48(1)$ \\
\hline$C(24)$ & $3658(2)$ & $6106(5)$ & $8355(2)$ & $59(1)$ \\
\hline$c(25)$ & $3737(2)$ & $7468(5)$ & $7982(2)$ & $58(1)$ \\
\hline$C(26)$ & $3226(2)$ & $8075(5)$ & $7614(2)$ & $52(1)$ \\
\hline$c(27)$ & 2618 (2) & $7378(4)$ & $7624(2)$ & $46(1)$ \\
\hline$C(28)$ & $3281(7)$ & $3881(9)$ & $9492(3)$ & $205(6)$ \\
\hline$C(29)$ & $2983(2)$ & $3858(6)$ & $8781(2)$ & $72(1)$ \\
\hline$c(30)$ & $3259(5)$ & $2523(6)$ & $8384(4)$ & $139(3)$ \\
\hline$c(31)$ & $2228(3)$ & $8041(7)$ & $6418(3)$ & $83(2)$ \\
\hline$c(32)$ & $2065(2)$ & $8082(5)$ & $7202(2)$ & $52(1)$ \\
\hline$C(33)$ & $1890(3)$ & $9671(6)$ & $7442(3)$ & $92(2)$ \\
\hline$C(34)$ & $2370(2)$ & $8651(5)$ & $9307(2)$ & $64(1)$ \\
\hline$C(35)$ & $2641(3)$ & $9422(6)$ & $9928(3)$ & $82(2)$ \\
\hline$C(36)$ & $2731(3)$ & $8115(6)$ & $10432(3)$ & $86(2)$ \\
\hline$c(37)$ & $2142(3)$ & $7120(7)$ & $10307(2)$ & $79(2)$ \\
\hline
\end{tabular}


Table S3. Bond lengths [A] and angles [deg] for 4.

\begin{tabular}{|c|c|}
\hline $\operatorname{Cr}(1)-N(2)$ & $1.946(3)$ \\
\hline $\operatorname{Cr}(1)-N(1)$ & $2.020(3)$ \\
\hline $\operatorname{Cr}(1)-N(3)$ & $2.020(3)$ \\
\hline $\operatorname{cr}(1)-0(1)$ & $2.083(2)$ \\
\hline$N(1)-C(14)$ & $1.375(4)$ \\
\hline$N(1)-C(1)$ & $1.442(5)$ \\
\hline$N(2)-C(15)$ & $1.361(4)$ \\
\hline$N(2)-C(19)$ & $1.382(4)$ \\
\hline$N(3)-C(20)$ & $1.382(4)$ \\
\hline$N(3)-C(22)$ & $1.427(4)$ \\
\hline $0(1)-C(37)$ & $1.442(5)$ \\
\hline $0(1)-C(34)$ & $1.453(5)$ \\
\hline$C(1)-C(6)$ & $1.400(5)$ \\
\hline$C(1)-C(2)$ & $1.400(5)$ \\
\hline$C(2)-C(3)$ & $1.392(6)$ \\
\hline$C(2)-C(8)$ & $1.524(6)$ \\
\hline$c(3)-c(4)$ & $1.361(6)$ \\
\hline$C(4)-C(5)$ & $1.399(7)$ \\
\hline$c(5)-c(6)$ & $1.382(5)$ \\
\hline$C(6)-C(11)$ & $1.531(5)$ \\
\hline$C(7)-C(8)$ & $1.507(7)$ \\
\hline$C(8)-C(9)$ & $1.515(8)$ \\
\hline$C(10)-C(11)$ & $1.518(6)$ \\
\hline$C(11)-C(12)$ & $1.517(5)$ \\
\hline$C(13)-C(14)$ & $1.421(5)$ \\
\hline
\end{tabular}




\begin{tabular}{|c|c|}
\hline$C(14)-C(15)$ & $1.440(5)$ \\
\hline$C(15)-C(16)$ & $1.410(5)$ \\
\hline$C(16)-C(17)$ & $1.405(6)$ \\
\hline$C(17)-C(18)$ & $1.376(6)$ \\
\hline$C(18)-C(19)$ & $1.400(5)$ \\
\hline$C(19)-C(20)$ & $1.424(5)$ \\
\hline$C(20)-C(21)$ & $1.447(5)$ \\
\hline$C(22)-C(23)$ & $1.397(5)$ \\
\hline$C(22)-C(27)$ & $1.410(5)$ \\
\hline$C(23)-C(24)$ & $1.387(6)$ \\
\hline$C(23)-C(29)$ & $1.522(6)$ \\
\hline$C(24)-C(25)$ & $1.384(6)$ \\
\hline$C(25)-C(26)$ & $1.373(6)$ \\
\hline$C(26)-C(27)$ & $1.399(5)$ \\
\hline$C(27)-C(32)$ & $1.527(5)$ \\
\hline$C(28)-C(29)$ & $1.490(9)$ \\
\hline$C(29)-C(30)$ & $1.493(8)$ \\
\hline$c(31)-c(32)$ & $1.532(6)$ \\
\hline$C(32)-C(33)$ & $1.492(6)$ \\
\hline$C(34)-C(35)$ & $1.469(6)$ \\
\hline$C(35)-C(36)$ & $1.495(7)$ \\
\hline$C(36)-C(37)$ & $1.511(7)$ \\
\hline$N(2)-C r(1)-N(1)$ & $79.00(11)$ \\
\hline$N(2)-C r(1)-N(3)$ & $78.90(11)$ \\
\hline$N(1)-C r(1)-N(3)$ & $157.86(11)$ \\
\hline$N(2)-\operatorname{Cr}(1)-O(1)$ & $178.49(12)$ \\
\hline$N(1)-\operatorname{Cr}(1)-0(1)$ & $101.19(11)$ \\
\hline
\end{tabular}




\begin{tabular}{|c|c|}
\hline$N(3)-\operatorname{Cr}(1)-0(1)$ & $100.93(11)$ \\
\hline$C(14)-N(1)-C(1)$ & $119.4(3)$ \\
\hline$C(14)-N(1)-C r(1)$ & $115.7(2)$ \\
\hline $\mathrm{C}(1)-\mathrm{N}(1)-\mathrm{Cr}(1)$ & $124.7(2)$ \\
\hline$C(15)-N(2)-C(19)$ & $122.3(3)$ \\
\hline$C(15)-N(2)-C r(1)$ & $118.6(2)$ \\
\hline$C(19)-N(2)-C r(1)$ & $119.2(2)$ \\
\hline$C(20)-N(3)-C(22)$ & $120.4(3)$ \\
\hline$C(20)-N(3)-C r(1)$ & $115.2(2)$ \\
\hline$C(22)-N(3)-C r(1)$ & $124.2(2)$ \\
\hline$C(37)-0(1)-C(34)$ & $109.8(3)$ \\
\hline$C(37)-0(1)-C r(1)$ & $125.3(3)$ \\
\hline$C(34)-0(1)-C r(1)$ & $124.9(2)$ \\
\hline$C(6)-C(1)-C(2)$ & $120.6(3)$ \\
\hline$C(6)-C(1)-N(1)$ & $120.4(3)$ \\
\hline$C(2)-C(1)-N(1)$ & $118.7(3)$ \\
\hline$C(3)-C(2)-C(1)$ & $118.4(4)$ \\
\hline$C(3)-C(2)-C(8)$ & $120.0(4)$ \\
\hline$C(1)-C(2)-C(8)$ & $121.5(4)$ \\
\hline$C(4)-C(3)-C(2)$ & $121.8(4)$ \\
\hline$C(3)-C(4)-C(5)$ & $119.4(4)$ \\
\hline$C(6)-C(5)-C(4)$ & $120.9(4)$ \\
\hline$C(5)-C(6)-C(1)$ & $118.9(3)$ \\
\hline$C(5)-C(6)-C(11)$ & $119.6(3)$ \\
\hline$C(1)-C(6)-C(11)$ & $121.4(3)$ \\
\hline$C(7)-C(8)-C(2)$ & $114.8(4)$ \\
\hline$C(7)-C(8)-C(9)$ & $108.6(4)$ \\
\hline$C(2)-C(8)-C(9)$ & $110.8(4)$ \\
\hline$C(10)-C(11)-C(6)$ & $112.6(3)$ \\
\hline
\end{tabular}




$$
\begin{aligned}
& C(10)-C(11)-C(12) \quad 111.1(4) \\
& C(6)-C(11)-C(12) \quad 111.7(3) \\
& \mathrm{N}(1)-\mathrm{C}(14)-\mathrm{C}(13) \quad 124.8(3) \\
& \mathrm{N}(1)-\mathrm{C}(14)-\mathrm{C}(15) \quad 113.0(3) \\
& C(13)-C(14)-C(15) \quad 122.2(3) \\
& \mathrm{N}(2)-\mathrm{C}(15)-\mathrm{C}(16) \quad 119.6(3) \\
& \mathrm{N}(2)-\mathrm{C}(15)-\mathrm{C}(14) \quad 113.6(3) \\
& C(16)-C(15)-C(14) \quad 126.8(3) \\
& \mathrm{C}(15)-\mathrm{C}(16)-\mathrm{C}(17) \quad 118.2(4) \\
& C(18)-C(17)-C(16) \quad 121.5(4) \\
& C(17)-C(18)-C(19) \quad 119.4(3) \\
& \mathrm{N}(2)-\mathrm{C}(19)-\mathrm{C}(18) \quad 119.1(3) \\
& \mathrm{N}(2)-\mathrm{C}(19)-\mathrm{C}(20) \quad 112.1(3) \\
& C(18)-C(19)-C(20) \quad 128.8(3) \\
& \mathrm{N}(3)-\mathrm{C}(20)-\mathrm{C}(19) \quad 114.6(3) \\
& \mathrm{N}(3)-\mathrm{C}(20)-\mathrm{C}(21) \quad 123.3(3) \\
& C(19)-C(20)-C(21) \quad 122.1(3) \\
& C(23)-C(22)-C(27) \quad 120.7(3) \\
& \mathrm{C}(23)-\mathrm{C}(22)-\mathrm{N}(3) \quad 119.8(3) \\
& \mathrm{C}(27)-\mathrm{C}(22)-\mathrm{N}(3) \quad 119.3(3) \\
& C(24)-C(23)-C(22) \quad 119.1(4) \\
& C(24)-C(23)-C(29) \quad 120.7(4) \\
& C(22)-C(23)-C(29) \quad 120.2(4) \\
& C(25)-C(24)-C(23) \quad 120.9(4) \\
& C(26)-C(25)-C(24) \quad 119.7(4) \\
& \mathrm{C}(25)-\mathrm{C}(26)-\mathrm{C}(27) \quad 121.6(4) \\
& \mathrm{C}(22)-\mathrm{C}(27)-\mathrm{C}(26) \quad 117.8(3) \\
& C(22)-C(27)-C(32) \quad 122.3(3)
\end{aligned}
$$




$$
\begin{array}{lr}
C(26)-C(27)-C(32) & 119.9(3) \\
C(28)-C(29)-C(30) & 108.3(6) \\
C(28)-C(29)-C(23) & 113.7(5) \\
C(30)-C(29)-C(23) & 111.3(4) \\
C(33)-C(32)-C(27) & 112.8(4) \\
C(33)-C(32)-C(31) & 112.0(4) \\
C(27)-C(32)-C(31) & 109.8(3) \\
0(1)-C(34)-C(35) & 105.6(4) \\
C(34)-C(35)-C(36) & 102.9(4) \\
C(35)-C(36)-C(37) & 103.2(4) \\
0(1)-C(37)-C(36) & 103.9(4)
\end{array}
$$

Symmetry transformations used to generate equivalent atoms: 
Table S4. Anisotropic displacement parameters $\left(A^{\wedge} 2 \times 10^{\wedge} 3\right)$ for 4 . The anisotropic displacement factor exponent takes the form:

$-2 \operatorname{pi} 2\left[h^{\wedge} 2 a^{* \wedge} \mathrm{U} 11+\ldots+2 h k a^{*} b^{*} \mathrm{U} 12\right]$

$\begin{array}{llllll}\text { U11 } & \text { U22 } & \text { U33 } & \text { U23 } & \text { U13 } & \text { U12 }\end{array}$

$\begin{array}{lrrrrrr}\mathrm{Cr}(1) & 34(1) & 40(1) & 36(1) & -6(1) & 0(1) & -6(1) \\ \mathrm{N}(1) & 35(2) & 42(2) & 39(2) & -7(1) & -1(1) & -2(1) \\ \mathrm{N}(2) & 39(2) & 33(1) & 35(2) & -1(1) & -4(1) & 5(1) \\ \mathrm{N}(3) & 37(2) & 40(2) & 40(2) & -2(1) & 2(1) & -3(1) \\ \mathrm{O}(1) & 5 \odot(2) & 71(2) & 47(2) & -12(1) & 0(1) & -25(1) \\ \mathrm{C}(1) & 32(2) & 48(2) & 44(2) & -5(2) & 6(2) & -2(2) \\ \mathrm{C}(2) & 6 \odot(2) & 44(2) & 58(2) & -8(2) & 8(2) & -4(2) \\ \mathrm{C}(3) & 84(3) & 51(2) & 62(3) & -16(2) & 17(2) & -10(2) \\ \mathrm{C}(4) & 73(3) & 75(3) & 53(3) & -22(2) & 15(2) & -21(3) \\ \mathrm{C}(5) & 59(3) & 75(3) & 40(2) & -8(2) & 8(2) & -14(2) \\ \mathrm{C}(6) & 32(2) & 55(2) & 43(2) & -5(2) & 6(2) & -1(2) \\ \mathrm{C}(7) & 84(3) & 69(3) & 117(4) & 30(3) & -5(3) & 3(3) \\ \mathrm{C}(8) & 94(4) & 48(2) & 60(3) & 0(2) & 11(2) & 7(2) \\ \mathrm{C}(9) & 132(6) & 116(5) & 95(4) & 29(4) & -48(4) & -44(4) \\ \mathrm{C}(10) & 78(3) & 70(3) & 87(3) & 4(3) & 30(3) & -11(3) \\ \mathrm{C}(11) & 46(2) & 54(2) & 44(2) & 2(2) & 4(2) & -2(2) \\ \mathrm{C}(12) & 61(3) & 57(2) & 97(3) & 12(3) & -2(2) & 2(2) \\ \mathrm{C}(13) & 38(2) & 82(3) & 64(3) & -20(2) & -1(2) & -5(2) \\ \mathrm{C}(14) & 34(2) & 45(2) & 50(2) & 2(2) & -4(2) & -1(2)\end{array}$




\begin{tabular}{|c|c|c|c|c|c|c|}
\hline$c(15)$ & $33(2)$ & $35(2)$ & $48(2)$ & $1(2)$ & $-7(2)$ & $-2(2)$ \\
\hline$C(16)$ & $35(2)$ & $49(2)$ & $63(3)$ & $-3(2)$ & $-12(2)$ & $-5(2)$ \\
\hline$c(17)$ & $54(3)$ & $66(3)$ & $68(3)$ & $-20(2)$ & $-15(2)$ & $-10(2)$ \\
\hline$c(18)$ & $61(3)$ & $42(2)$ & $46(2)$ & $-14(2)$ & $-3(2)$ & $\Theta(2)$ \\
\hline$c(19)$ & $46(2)$ & $32(2)$ & $39(2)$ & $\odot(2)$ & $-6(2)$ & $5(2)$ \\
\hline$c(20)$ & $55(2)$ & $32(2)$ & $39(2)$ & $-3(2)$ & $-2(2)$ & $1(2)$ \\
\hline$C(21)$ & $57(2)$ & $56(2)$ & $51(2)$ & $-14(2)$ & $\odot(2)$ & $2(2)$ \\
\hline$c(22)$ & $34(2)$ & $40(2)$ & $40(2)$ & $-6(2)$ & $4(1)$ & $-1(2)$ \\
\hline$c(23)$ & $45(2)$ & $55(2)$ & $44(2)$ & $-1(2)$ & $3(2)$ & $9(2)$ \\
\hline$c(24)$ & $4 \odot(2)$ & $75(3)$ & $61(2)$ & $2(2)$ & $-4(2)$ & $13(2)$ \\
\hline$C(25)$ & $42(2)$ & $65(3)$ & $66(3)$ & $-5(2)$ & $2(2)$ & $-9(2)$ \\
\hline$C(26)$ & $47(2)$ & $51(2)$ & $57(2)$ & $4(2)$ & $10(2)$ & $-5(2)$ \\
\hline$C(27)$ & $41(2)$ & $47(2)$ & $50(2)$ & $-5(2)$ & $5(2)$ & $4(2)$ \\
\hline$C(28)$ & $470(20)$ & $78(4)$ & $71(4)$ & $18(4)$ & $-45(7)$ & $-11(8)$ \\
\hline$C(29)$ & $70(3)$ & $66(3)$ & $81(3)$ & $25(3)$ & $2(2)$ & $13(2)$ \\
\hline$c(30)$ & $243(10)$ & $52(3)$ & $121(6)$ & $6(3)$ & $-1(6)$ & $10(4)$ \\
\hline$c(31)$ & $73(3)$ & $111(4)$ & $64(3)$ & $21(3)$ & $-1(2)$ & $16(3)$ \\
\hline$c(32)$ & $45(2)$ & $54(2)$ & $56(2)$ & $12(2)$ & $2(2)$ & $6(2)$ \\
\hline$c(33)$ & $99(4)$ & $62(3)$ & $113(5)$ & $-14(3)$ & $-40(4)$ & $27(3)$ \\
\hline$c(34)$ & $7 \odot(3)$ & $63(3)$ & $58(2)$ & $-7(2)$ & $-2(2)$ & $-23(2)$ \\
\hline$c(35)$ & $95(4)$ & $76(3)$ & $76(3)$ & $1(2)$ & $-26(3)$ & $-30(3)$ \\
\hline$c(36)$ & $94(4)$ & $91(4)$ & $73(3)$ & $10(3)$ & $-35(3)$ & $-35(3)$ \\
\hline$c(37)$ & $86(3)$ & $105(4)$ & $46(3)$ & $\odot(2)$ & $-7(2)$ & $-44(3)$ \\
\hline
\end{tabular}


Table S5. Hydrogen coordinates $(x 10 \wedge 4)$ and isotropic displacement parameters $\left(A^{\wedge} 2 \times 10^{\wedge} 3\right)$ for 4 .

$\begin{array}{llll}x & y & z & U(\text { eq })\end{array}$

\begin{tabular}{|c|c|c|c|c|}
\hline$H(3 A)$ & 489 & 10654 & 10761 & 79 \\
\hline$H(4 A)$ & 567 & 9488 & 11844 & 81 \\
\hline$H(5 A)$ & 565 & 6779 & 11926 & 70 \\
\hline$H(7 A)$ & 1244 & 10852 & 9532 & 135 \\
\hline$H(7 B)$ & 601 & 11762 & 9746 & 135 \\
\hline$H(7 C)$ & 753 & 11423 & 8938 & 135 \\
\hline$H(8 A)$ & 616 & 8794 & 9105 & 81 \\
\hline$H(9 A)$ & -294 & 10296 & 8820 & 172 \\
\hline$H(9 B)$ & -471 & 10440 & 9634 & 172 \\
\hline$H(9 C)$ & -497 & 8789 & 9255 & 172 \\
\hline$H(10 A)$ & -364 & 4408 & 11425 & 118 \\
\hline$H(10 B)$ & 220 & 4340 & 11974 & 118 \\
\hline$H(10 C)$ & 57 & 2864 & 11498 & 118 \\
\hline$H(11 A)$ & 375 & 4155 & 10492 & 58 \\
\hline$H(12 A)$ & 1194 & 2853 & 11114 & 107 \\
\hline$H(12 B)$ & 1383 & 4436 & 11498 & 107 \\
\hline$H(12 C)$ & 1478 & 4266 & 10668 & 107 \\
\hline$H(13 A)$ & -671 & 5994 & 10079 & 92 \\
\hline$H(13 B)$ & -948 & 4857 & 9456 & 92 \\
\hline$H(16 A)$ & -723 & 3483 & 8491 & 59 \\
\hline
\end{tabular}




\begin{tabular}{|c|c|c|c|c|}
\hline$H(17 A)$ & -370 & 2299 & 7445 & 75 \\
\hline$H(18 A)$ & 680 & 2620 & 7050 & 60 \\
\hline$H(21 A)$ & 2364 & 4403 & 7019 & 82 \\
\hline$H(21 B)$ & 1682 & 4370 & 6626 & 82 \\
\hline$H(21 C)$ & 1937 & 2861 & 7027 & 82 \\
\hline$H(24 A)$ & 4015 & 5673 & 8597 & 70 \\
\hline$H(25 A)$ & 4142 & 7980 & 7980 & 69 \\
\hline$H(26 A)$ & 3288 & 8991 & 7347 & 62 \\
\hline$H(28 A)$ & 3206 & 2885 & 9724 & 308 \\
\hline $\mathrm{H}(28 \mathrm{~B})$ & 3087 & 4711 & 9771 & 308 \\
\hline$H(28 C)$ & 3746 & 4061 & 9449 & 308 \\
\hline$H(29 A)$ & 2510 & 3669 & 8840 & 87 \\
\hline$H(30 A)$ & 3193 & 1568 & 8652 & 208 \\
\hline$H(3 \odot B)$ & 3721 & 2689 & 8311 & 208 \\
\hline$H(30 \mathrm{C})$ & 3042 & 2436 & 7929 & 208 \\
\hline$H(31 A)$ & 1873 & 8502 & 6150 & 124 \\
\hline$H(31 B)$ & 2290 & 6965 & 6269 & 124 \\
\hline$H(31 C)$ & 2625 & 8628 & 6334 & 124 \\
\hline$H(32 A)$ & 1678 & 7410 & 7274 & 62 \\
\hline$H(33 A)$ & 1794 & 9651 & 7945 & 137 \\
\hline$H(33 B)$ & 1510 & 10031 & 7184 & 137 \\
\hline$H(33 C)$ & 2252 & 10375 & 7355 & 137 \\
\hline$H(34 A)$ & 2090 & 9370 & 9041 & 76 \\
\hline$H(34 B)$ & 2719 & 8282 & 8994 & 76 \\
\hline$H(35 A)$ & 2338 & 10205 & 10117 & 98 \\
\hline$H(35 B)$ & 3057 & 9928 & 9817 & 98 \\
\hline$H(36 A)$ & 2745 & 8494 & 10922 & 103 \\
\hline$H(36 B)$ & 3132 & 7537 & 10330 & 103 \\
\hline
\end{tabular}




\begin{tabular}{|c|c|c|c|c|}
\hline $\mathrm{H}(37 \mathrm{~A})$ & 2237 & 6017 & 10404 & 95 \\
\hline H( 37B ) & 1778 & 7455 & 10607 & 95 \\
\hline
\end{tabular}


Table s6. Torsion angles [deg] for 4.

$$
\begin{aligned}
& N(2)-C r(1)-N(1)-C(14) \\
& -1.5(2) \\
& \mathrm{N}(3)-\mathrm{Cr}(1)-\mathrm{N}(1)-\mathrm{C}(14) \\
& \text { 2.1(5) } \\
& O(1)-\operatorname{Cr}(1)-N(1)-C(14) \\
& -180 . \odot(2) \\
& N(2)-C r(1)-N(1)-C(1) \\
& -177.8(3) \\
& \mathrm{N}(3)-\operatorname{Cr}(1)-\mathrm{N}(1)-\mathrm{C}(1) \\
& -174.1(3) \\
& \mathrm{O}(1)-\mathrm{Cr}(1)-\mathrm{N}(1)-\mathrm{C}(1) \\
& 3.8(3) \\
& \mathrm{N}(1)-\mathrm{Cr}(1)-\mathrm{N}(2)-\mathrm{C}(15) \\
& 0.1(2) \\
& \mathrm{N}(3)-\mathrm{Cr}(1)-\mathrm{N}(2)-\mathrm{C}(15) \\
& O(1)-\operatorname{Cr}(1)-N(2)-C(15) \\
& \mathrm{N}(1)-\mathrm{Cr}(1)-\mathrm{N}(2)-\mathrm{C}(19) \\
& \mathrm{N}(3)-\mathrm{Cr}(1)-\mathrm{N}(2)-\mathrm{C}(19) \\
& O(1)-\operatorname{Cr}(1)-N(2)-C(19) \\
& \mathrm{N}(2)-\mathrm{Cr}(1)-\mathrm{N}(3)-\mathrm{C}(20) \\
& N(1)-\operatorname{Cr}(1)-N(3)-C(20) \\
& O(1)-C r(1)-N(3)-C(20) \\
& \mathrm{N}(2)-\mathrm{Cr}(1)-\mathrm{N}(3)-\mathrm{C}(22) \\
& \mathrm{N}(1)-\mathrm{Cr}(1)-\mathrm{N}(3)-\mathrm{C}(22) \\
& O(1)-\operatorname{Cr}(1)-N(3)-C(22) \\
& \mathrm{N}(2)-\operatorname{Cr}(1)-0(1)-\mathrm{C}(37) \\
& \mathrm{N}(1)-\operatorname{Cr}(1)-0(1)-\mathrm{C}(37) \\
& 52.9(4) \\
& \mathrm{N}(3)-\mathrm{Cr}(1)-0(1)-\mathrm{C}(37) \\
& -127.9(4) \\
& N(2)-\operatorname{Cr}(1)-0(1)-C(34) \\
& \text { 136(4) } \\
& N(1)-\operatorname{Cr}(1)-0(1)-C(34) \\
& -126.9(3) \\
& N(3)-\operatorname{Cr}(1)-O(1)-C(34) \\
& 52.3(3) \\
& C(14)-N(1)-C(1)-C(6) \\
& 80 . \odot(4)
\end{aligned}
$$




$$
\begin{aligned}
& \operatorname{Cr}(1)-N(1)-C(1)-C(6) \quad-103.9(3) \\
& C(14)-N(1)-C(1)-C(2) \quad-105.9(4) \\
& \begin{array}{ll}
\operatorname{Cr}(1)-N(1)-C(1)-C(2) & 70.2(4)
\end{array} \\
& C(6)-C(1)-C(2)-C(3) \quad 0.9(6) \\
& N(1)-C(1)-C(2)-C(3) \quad-173.2(4) \\
& C(6)-C(1)-C(2)-C(8) \quad-177.1(4) \\
& N(1)-C(1)-C(2)-C(8) \quad 8.8(5) \\
& C(1)-C(2)-C(3)-C(4) \quad 1.0(7) \\
& C(8)-C(2)-C(3)-C(4) \quad 179.0(4) \\
& C(2)-C(3)-C(4)-C(5) \quad-1.9(7) \\
& C(3)-C(4)-C(5)-C(6) \quad 0.9(7) \\
& C(4)-C(5)-C(6)-C(1) \quad 1.0(6) \\
& C(4)-C(5)-C(6)-C(11) \quad 179.3(4) \\
& C(2)-C(1)-C(6)-C(5) \quad-1.9(5) \\
& \begin{array}{ll}
N(1)-C(1)-C(6)-C(5) & 172.1(3)
\end{array} \\
& C(2)-C(1)-C(6)-C(11) \quad 179.9 \text { (3) } \\
& N(1)-C(1)-C(6)-C(11) \quad-6.1(5) \\
& C(3)-C(2)-C(8)-C(7) \quad 35.9(6) \\
& \mathrm{C}(1)-\mathrm{C}(2)-\mathrm{C}(8)-\mathrm{C}(7) \quad-146.1(4) \\
& C(3)-C(2)-C(8)-C(9) \quad-87.5(6) \\
& C(1)-C(2)-C(8)-C(9) \quad 90.4(5) \\
& \mathrm{C}(5)-\mathrm{C}(6)-\mathrm{C}(11)-\mathrm{C}(10) \quad 46.3(5) \\
& C(1)-C(6)-C(11)-C(10) \quad-135.4(4) \\
& C(5)-C(6)-C(11)-C(12) \quad-79.5(5) \\
& C(1)-C(6)-C(11)-C(12) \quad 98.8(4) \\
& \mathrm{C}(1)-\mathrm{N}(1)-\mathrm{C}(14)-\mathrm{C}(13) \quad 0.2(6) \\
& \mathrm{Cr}(1)-\mathrm{N}(1)-\mathrm{C}(14)-\mathrm{C}(13) \quad-176.2(3) \\
& C(1)-N(1)-C(14)-C(15) \quad 179 . \odot(3)
\end{aligned}
$$




$$
\begin{aligned}
& \operatorname{Cr}(1)-N(1)-C(14)-C(15) \quad 2.5(4) \\
& \mathrm{C}(19)-\mathrm{N}(2)-\mathrm{C}(15)-\mathrm{C}(16) \quad 1.1(5) \\
& \operatorname{Cr}(1)-\mathrm{N}(2)-\mathrm{C}(15)-\mathrm{C}(16) \quad-178.6(3) \\
& \mathrm{C}(19)-\mathrm{N}(2)-\mathrm{C}(15)-\mathrm{C}(14) \quad-179.1 \text { (3) } \\
& \operatorname{Cr}(1)-N(2)-C(15)-C(14) \quad 1.2(4) \\
& \mathrm{N}(1)-\mathrm{C}(14)-\mathrm{C}(15)-\mathrm{N}(2) \quad-2.4(4) \\
& \mathrm{C}(13)-\mathrm{C}(14)-\mathrm{C}(15)-\mathrm{N}(2) \quad 176.4(3) \\
& N(1)-C(14)-C(15)-C(16) \quad 177.4(3) \\
& \mathrm{C}(13)-\mathrm{C}(14)-\mathrm{C}(15)-\mathrm{C}(16) \quad-3.8(6) \\
& N(2)-C(15)-C(16)-C(17) \quad-0.3(5) \\
& C(14)-C(15)-C(16)-C(17) \quad 179.9(4) \\
& C(15)-C(16)-C(17)-C(18) \quad 0.7(6) \\
& C(16)-C(17)-C(18)-C(19) \quad-1.8(6) \\
& \mathrm{C}(15)-\mathrm{N}(2)-\mathrm{C}(19)-\mathrm{C}(18) \quad-2.2(5) \\
& \operatorname{Cr}(1)-\mathrm{N}(2)-\mathrm{C}(19)-\mathrm{C}(18) \quad 177.6(2) \\
& C(15)-N(2)-C(19)-C(20) \quad 177.6(3) \\
& \mathrm{Cr}(1)-\mathrm{N}(2)-\mathrm{C}(19)-\mathrm{C}(20) \quad-2.6(4) \\
& \mathrm{C}(17)-\mathrm{C}(18)-\mathrm{C}(19)-\mathrm{N}(2) \quad 2.4(5) \\
& C(17)-C(18)-C(19)-C(20) \quad-177.3(4) \\
& \mathrm{C}(22)-\mathrm{N}(3)-\mathrm{C}(20)-\mathrm{C}(19) \quad-176.1 \text { (3) } \\
& \operatorname{Cr}(1)-\mathrm{N}(3)-\mathrm{C}(20)-\mathrm{C}(19) \quad-0.7(4) \\
& \mathrm{C}(22)-\mathrm{N}(3)-\mathrm{C}(20)-\mathrm{C}(21) \quad 3.1(5) \\
& \mathrm{Cr}(1)-\mathrm{N}(3)-\mathrm{C}(20)-\mathrm{C}(21) \quad 178.5(3) \\
& \mathrm{N}(2)-\mathrm{C}(19)-\mathrm{C}(20)-\mathrm{N}(3) \quad 2.1(4) \\
& \mathrm{C}(18)-\mathrm{C}(19)-\mathrm{C}(20)-\mathrm{N}(3) \quad-178.2(3) \\
& N(2)-C(19)-C(20)-C(21) \quad-177.2(3) \\
& C(18)-C(19)-C(20)-C(21) \quad 2.6(6) \\
& \mathrm{C}(20)-\mathrm{N}(3)-\mathrm{C}(22)-\mathrm{C}(23) \quad-102.5(4) \\
& \mathrm{Cr}(1)-\mathrm{N}(3)-\mathrm{C}(22)-\mathrm{C}(23) \quad 82.6(4)
\end{aligned}
$$




$$
\begin{aligned}
& C(20)-N(3)-C(22)-C(27) \quad 83.2(4) \\
& \operatorname{Cr}(1)-\mathrm{N}(3)-\mathrm{C}(22)-\mathrm{C}(27) \quad-91.8(4) \\
& C(27)-C(22)-C(23)-C(24) \quad 5.5(5) \\
& \mathrm{N}(3)-\mathrm{C}(22)-\mathrm{C}(23)-\mathrm{C}(24) \quad-168.7 \text { (3) } \\
& C(27)-C(22)-C(23)-C(29) \quad-175.6(3) \\
& N(3)-C(22)-C(23)-C(29) \quad 10.2(5) \\
& C(22)-C(23)-C(24)-C(25) \quad-2.0(6) \\
& C(29)-C(23)-C(24)-C(25) \quad 179.1(4) \\
& C(23)-C(24)-C(25)-C(26) \quad-1.8(6) \\
& C(24)-C(25)-C(26)-C(27) \quad 2.1(6) \\
& C(23)-C(22)-C(27)-C(26) \quad-5.2(5) \\
& \mathrm{N}(3)-\mathrm{C}(22)-\mathrm{C}(27)-\mathrm{C}(26) \quad 169.1(3) \\
& C(23)-C(22)-C(27)-C(32) \quad 175.6(3) \\
& \mathrm{N}(3)-\mathrm{C}(22)-\mathrm{C}(27)-\mathrm{C}(32) \quad-10.2(5) \\
& \mathrm{C}(25)-\mathrm{C}(26)-\mathrm{C}(27)-\mathrm{C}(22) \quad 1.4(6) \\
& C(25)-C(26)-C(27)-C(32) \quad-179.4(4) \\
& C(24)-C(23)-C(29)-C(28) \quad 45.9(8) \\
& C(22)-C(23)-C(29)-C(28) \quad-132.9(6) \\
& C(24)-C(23)-C(29)-C(30) \quad-76.6(6) \\
& C(22)-C(23)-C(29)-C(30) \quad 104.5(6) \\
& C(22)-C(27)-C(32)-C(33) \quad 116.5(5) \\
& C(26)-C(27)-C(32)-C(33) \quad-62.7(5) \\
& C(22)-C(27)-C(32)-C(31) \quad-117.8(4) \\
& C(26)-C(27)-C(32)-C(31) \quad 63.0(5) \\
& \mathrm{C}(37)-0(1)-\mathrm{C}(34)-\mathrm{C}(35) \quad-12.2(5) \\
& \begin{array}{ll}
\operatorname{Cr}(1)-0(1)-C(34)-C(35) & 167.6(3)
\end{array} \\
& 0(1)-C(34)-C(35)-C(36) \quad 31.3(5) \\
& C(34)-C(35)-C(36)-C(37) \quad-38.4(6)
\end{aligned}
$$



$C(34)-0(1)-C(37)-C(36)$
$-12 . \odot(6)$
$\operatorname{cr}(1)-0(1)-C(37)-C(36)$
$168.2(3)$
$C(35)-C(36)-C(37)-0(1)$
$31 . \odot(6)$

Symmetry transformations used to generate equivalent atoms: 


\section{Complex 5}


Table S7. Crystal data and structure refinement for $\mathbf{5}$.

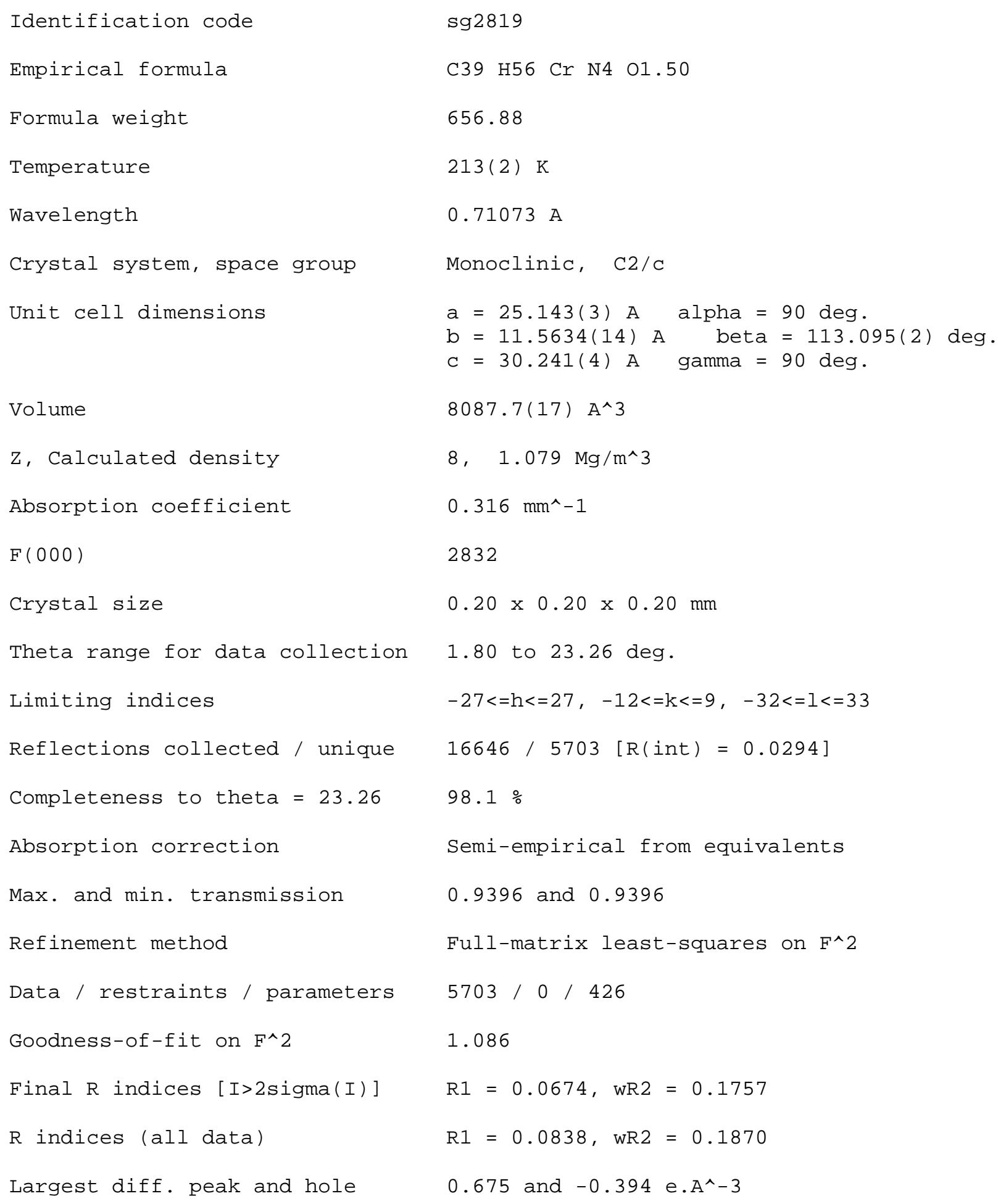


Table s8. Atomic coordinates $(x 10 \wedge 4)$ and equivalent isotropic displacement parameters $\left(A^{\wedge} 2 \times 10 \wedge 3\right)$ for 5 .

$U(e q)$ is defined as one third of the trace of the orthogonalized Uij tensor.

\begin{tabular}{|c|c|c|c|c|}
\hline & $x$ & $y$ & z & $\mathrm{U}(\mathrm{eq})$ \\
\hline $\operatorname{cr}(1)$ & $5893(1)$ & $6646(1)$ & $8143(1)$ & $31(1)$ \\
\hline$N(1)$ & $6599(1)$ & $7315(2)$ & $7994(1)$ & $30(1)$ \\
\hline$N(2)$ & $6135(1)$ & $5364(2)$ & $7853(1)$ & $31(1)$ \\
\hline$N(3)$ & $5716(1)$ & $5201(3)$ & $8475(1)$ & $32(1)$ \\
\hline$N(4)$ & $5227(1)$ & $7090(2)$ & $7684(1)$ & $28(1)$ \\
\hline$c(1)$ & $7176(2)$ & $7024(4)$ & $7487(2)$ & $49(1)$ \\
\hline$c(2)$ & $6766(2)$ & $6635(3)$ & $7714(1)$ & $34(1)$ \\
\hline$C(3)$ & $6524(2)$ & $5504(3)$ & $7641(1)$ & $32(1)$ \\
\hline$C(4)$ & $6666(2)$ & $4592(4)$ & $7412(2)$ & $42(1)$ \\
\hline$C(5)$ & $6409(2)$ & $3516(3)$ & $7396(2)$ & $46(1)$ \\
\hline$C(6)$ & $6043(2)$ & $3360(3)$ & $7633(2)$ & $45(1)$ \\
\hline$c(7)$ & $5924(2)$ & $4255(3)$ & $7882(1)$ & $33(1)$ \\
\hline$C(8)$ & $5676(2)$ & $4198(3)$ & $8225(1)$ & $35(1)$ \\
\hline$C(9)$ & $5384(2)$ & $3139(4)$ & $8313(2)$ & $49(1)$ \\
\hline$c(10)$ & $6651(2)$ & $9392(3)$ & $7835(2)$ & $37(1)$ \\
\hline$C(11)$ & $6899(2)$ & $10473(4)$ & $8002(2)$ & $46(1)$ \\
\hline$C(12)$ & $7347(2)$ & $10596(4)$ & $8442(2)$ & $53(1)$ \\
\hline$c(13)$ & $7563(2)$ & $9633(4)$ & $8723(2)$ & $53(1)$ \\
\hline$c(14)$ & $7330(2)$ & $8539(4)$ & $8580(2)$ & $41(1)$ \\
\hline$C(15)$ & $6865(2)$ & $8429(3)$ & $8130(1)$ & $34(1)$ \\
\hline$C(16)$ & $6172(2)$ & $9305(4)$ & $7338(2)$ & $46(1)$ \\
\hline$C(17)$ & $6378(3)$ & $9739(6)$ & $6948(2)$ & $79(2)$ \\
\hline$C(18)$ & $5633(2)$ & $9954(5)$ & $7300(2)$ & $71(2)$ \\
\hline$C(19)$ & $7579(2)$ & $7495(4)$ & $8898(2)$ & $55(1)$ \\
\hline$C(20)$ & $8011(3)$ & $6860(6)$ & $8748(3)$ & $97(2)$ \\
\hline$C(21)$ & $7867(3)$ & $7801(6)$ & $9435(2)$ & $82(2)$ \\
\hline$C(22)$ & $5242(2)$ & $5457(4)$ & $9048(2)$ & $40(1)$ \\
\hline$C(23)$ & $5301(2)$ & $5493(4)$ & $9521(2)$ & $52(1)$ \\
\hline$c(24)$ & $5804(2)$ & $5155(5)$ & $9891(2)$ & $58(1)$ \\
\hline$C(25)$ & $6253(2)$ & $4745(4)$ & $9789(2)$ & $53(1)$ \\
\hline$C(26)$ & $6226(2)$ & $4706(3)$ & $9317(2)$ & $42(1)$ \\
\hline$C(27)$ & $5717(2)$ & $5107(3)$ & 8948(1) & $35(1)$ \\
\hline$C(28)$ & $4665(2)$ & $5774(4)$ & $8649(2)$ & $49(1)$ \\
\hline$C(29)$ & $4594(2)$ & $7070(5)$ & $8591(2)$ & $75(2)$ \\
\hline$C(30)$ & $4157(2)$ & $5265(5)$ & $8742(2)$ & $66(1)$ \\
\hline$C(31)$ & $6747(2)$ & $4282(4)$ & $9230(2)$ & $58(1)$ \\
\hline$C(32)$ & $7306(2)$ & $4868(5)$ & $9563(2)$ & $73(2)$ \\
\hline$c(33)$ & $6817(3)$ & $3007(5)$ & $9279(3)$ & $98(2)$ \\
\hline $0(1)$ & $6043(1)$ & $7895(2)$ & $8721(1)$ & $39(1)$ \\
\hline$C(34)$ & $5870(2)$ & $9084(4)$ & $8590(2)$ & $52(1)$ \\
\hline$c(36)$ & $6217(8)$ & $8984(13)$ & $9442(7)$ & $63(5)$ \\
\hline$C(35)$ & $5794(4)$ & $9596(7)$ & $9010(3)$ & $66(2)$ \\
\hline$C\left(35^{\prime}\right)$ & $6158(11)$ & $9796(18)$ & $9039(9)$ & $72(7)$ \\
\hline$c\left(36^{\prime}\right)$ & $6193(19)$ & $8840(30)$ & $9405(10)$ & $190(20)$ \\
\hline$C(37)$ & $6256(2)$ & $7767(4)$ & $9238(2)$ & $52(1)$ \\
\hline$c(38)$ & $6516(7)$ & $1837(14)$ & $827(6)$ & $110(5)$ \\
\hline
\end{tabular}




$\begin{array}{lllrr}C(39) & 6162(7) & 1492(15) & 442(6) & 115(5) \\ O(2) & 5643(4) & 1890(7) & 207(3) & 84(2) \\ C(40) & 5371(6) & 1514(12) & -194(5) & 90(4) \\ C(41) & 4836(9) & 2084(18) & -510(8) & 148(7)\end{array}$


Table s9. Bond lengths [A] and angles [deg] for 5 .

\begin{tabular}{|c|c|}
\hline $\operatorname{Cr}(1)-N(4)$ & $1.781(3)$ \\
\hline $\operatorname{Cr}(1)-N(2)$ & $1.940(3)$ \\
\hline $\operatorname{Cr}(1)-N(3)$ & $2.083(3)$ \\
\hline $\operatorname{Cr}(1)-N(1)$ & $2.141(3)$ \\
\hline $\operatorname{cr}(1)-0(1)$ & $2.182(3)$ \\
\hline$N(1)-C(2)$ & $1.338(5)$ \\
\hline$N(1)-C(15)$ & $1.436(5)$ \\
\hline$N(2)-C(3)$ & $1.372(5)$ \\
\hline$N(2)-C(7)$ & $1.404(5)$ \\
\hline$N(3)-C(8)$ & $1.366(5)$ \\
\hline$N(3)-C(27)$ & $1.434(5)$ \\
\hline$N(4)-N(4) \# 1$ & $1.241(6)$ \\
\hline$C(1)-C(2)$ & $1.512(5)$ \\
\hline$c(2)-c(3)$ & $1.424(5)$ \\
\hline$C(3)-c(4)$ & $1.382(5)$ \\
\hline$C(4)-C(5)$ & $1.394(6)$ \\
\hline$C(5)-C(6)$ & $1.383(6)$ \\
\hline$C(6)-C(7)$ & $1.380(6)$ \\
\hline$C(7)-C(8)$ & $1.406(5)$ \\
\hline$C(8)-C(9)$ & $1.505(6)$ \\
\hline$C(10)-C(15)$ & $1.396(6)$ \\
\hline$C(10)-C(11)$ & $1.400(6)$ \\
\hline$C(10)-C(16)$ & $1.517(6)$ \\
\hline$C(11)-C(12)$ & $1.372(6)$ \\
\hline$C(12)-C(13)$ & $1.378(7)$ \\
\hline$C(13)-C(14)$ & $1.390(6)$ \\
\hline$C(14)-C(15)$ & $1.409(6)$ \\
\hline$C(14)-C(19)$ & $1.517(6)$ \\
\hline$C(16)-C(18)$ & $1.515(7)$ \\
\hline$C(16)-C(17)$ & $1.545(7)$ \\
\hline$C(19)-C(20)$ & $1.519(8)$ \\
\hline$C(19)-C(21)$ & $1.539(7)$ \\
\hline$C(22)-C(23)$ & $1.381(6)$ \\
\hline$C(22)-C(27)$ & $1.400(6)$ \\
\hline$C(22)-C(28)$ & $1.524(6)$ \\
\hline$C(23)-C(24)$ & $1.377(7)$ \\
\hline$C(24)-C(25)$ & $1.366(7)$ \\
\hline$C(25)-C(26)$ & $1.401(6)$ \\
\hline$C(26)-C(27)$ & $1.408(6)$ \\
\hline$C(26)-C(31)$ & $1.517(6)$ \\
\hline$C(28)-C(29)$ & $1.512(8)$ \\
\hline$C(28)-C(30)$ & $1.527(6)$ \\
\hline$c(31)-c(33)$ & $1.485(8)$ \\
\hline$C(31)-C(32)$ & $1.529(7)$ \\
\hline $0(1)-C(34)$ & $1.450(5)$ \\
\hline$O(1)-C(37)$ & $1.448(5)$ \\
\hline$C(34)-C\left(35^{\prime}\right)$ & $1.51(2)$ \\
\hline$C(34)-C(35)$ & $1.480(9)$ \\
\hline$C(36)-C(37)$ & $1.555(16)$ \\
\hline$C(36)-C(35)$ & $1.50(2)$ \\
\hline$C\left(35^{\prime}\right)-C\left(36^{\prime}\right)$ & $1.54(4)$ \\
\hline$C\left(36^{\prime}\right)-C(37)$ & $1.38(3)$ \\
\hline$C(38)-C(39)$ & $1.22(2)$ \\
\hline
\end{tabular}




\begin{tabular}{|c|c|}
\hline$C(39)-0(2)$ & $1.301(17)$ \\
\hline $0(2)-C(40)$ & $1.215(15)$ \\
\hline$c(40)-C(41)$ & $1.46(2)$ \\
\hline$N(4)-C r(1)-N(2)$ & $103.46(13)$ \\
\hline$N(4)-C r(1)-N(3)$ & $107.13(13)$ \\
\hline$N(2)-C r(1)-N(3)$ & $76.61(12)$ \\
\hline$N(4)-C r(1)-N(1)$ & $109.51(12)$ \\
\hline$N(2)-C r(1)-N(1)$ & $75.33(12)$ \\
\hline$N(3)-C r(1)-N(1)$ & $137.88(12)$ \\
\hline$N(4)-\operatorname{Cr}(1)-0(1)$ & $104.41(12)$ \\
\hline$N(2)-C r(1)-0(1)$ & $151.88(12)$ \\
\hline$N(3)-C r(1)-0(1)$ & $98.56(11)$ \\
\hline$N(1)-C r(1)-0(1)$ & $91.84(11)$ \\
\hline$C(2)-N(1)-C(15)$ & $118.8(3)$ \\
\hline$C(2)-N(1)-C r(1)$ & $114.5(2)$ \\
\hline $\mathrm{C}(15)-\mathrm{N}(1)-\mathrm{Cr}(1)$ & $126.5(2)$ \\
\hline$C(3)-N(2)-C(7)$ & $119.5(3)$ \\
\hline $\mathrm{C}(3)-\mathrm{N}(2)-\mathrm{Cr}(1)$ & $121.9(2)$ \\
\hline $\mathrm{C}(7)-\mathrm{N}(2)-\mathrm{Cr}(1)$ & $118.5(2)$ \\
\hline$C(8)-N(3)-C(27)$ & $117.3(3)$ \\
\hline $\mathrm{C}(8)-\mathrm{N}(3)-\mathrm{Cr}(1)$ & $113.5(2)$ \\
\hline $\mathrm{C}(27)-\mathrm{N}(3)-\mathrm{Cr}(1)$ & $128.5(2)$ \\
\hline$N(4) \# 1-N(4)-\operatorname{Cr}(1)$ & $161.95(15)$ \\
\hline$N(1)-C(2)-C(3)$ & $114.6(3)$ \\
\hline$N(1)-C(2)-C(1)$ & $123.5(3)$ \\
\hline$c(3)-c(2)-c(1)$ & $121.9(3)$ \\
\hline$N(2)-C(3)-C(4)$ & $121.0(3)$ \\
\hline$N(2)-C(3)-C(2)$ & $112.1(3)$ \\
\hline$C(4)-C(3)-C(2)$ & $126.8(3)$ \\
\hline$C(3)-c(4)-C(5)$ & $119.4(4)$ \\
\hline$C(6)-C(5)-C(4)$ & $119.5(4)$ \\
\hline$C(5)-C(6)-C(7)$ & $121.0(4)$ \\
\hline$C(6)-C(7)-N(2)$ & $118.9(3)$ \\
\hline$C(6)-C(7)-C(8)$ & $128.4(4)$ \\
\hline$N(2)-C(7)-C(8)$ & $112.1(3)$ \\
\hline$N(3)-C(8)-C(7)$ & $114.2(3)$ \\
\hline$N(3)-C(8)-C(9)$ & $121.9(3)$ \\
\hline$C(7)-C(8)-C(9)$ & $123.9(4)$ \\
\hline$C(15)-C(10)-C(11)$ & $118.4(4)$ \\
\hline$C(15)-C(10)-C(16)$ & $122.5(3)$ \\
\hline$C(11)-C(10)-C(16)$ & $119.0(4)$ \\
\hline$C(12)-C(11)-C(10)$ & $121.5(4)$ \\
\hline$C(11)-C(12)-C(13)$ & $119.3(4)$ \\
\hline$C(12)-C(13)-C(14)$ & $121.9(4)$ \\
\hline$C(13)-C(14)-C(15)$ & $118.1(4)$ \\
\hline$C(13)-C(14)-C(19)$ & $120.5(4)$ \\
\hline$C(15)-C(14)-C(19)$ & $121.3(4)$ \\
\hline$C(10)-C(15)-C(14)$ & $120.7(3)$ \\
\hline$C(10)-C(15)-N(1)$ & $120.8(3)$ \\
\hline $\mathrm{C}(14)-\mathrm{C}(15)-\mathrm{N}(1)$ & $118.4(3)$ \\
\hline$C(18)-C(16)-C(10)$ & $112.4(4)$ \\
\hline$C(18)-C(16)-C(17)$ & $109.8(4)$ \\
\hline$C(10)-C(16)-C(17)$ & $111.1(4)$ \\
\hline$C(14)-C(19)-C(20)$ & $111.2(4)$ \\
\hline$C(14)-C(19)-C(21)$ & $113.1(4)$ \\
\hline$C(20)-C(19)-C(21)$ & $109.4(5)$ \\
\hline
\end{tabular}




$\begin{array}{lc}C(23)-C(22)-C(27) & 118.6(4) \\ C(23)-C(22)-C(28) & 119.6(4) \\ C(27)-C(22)-C(28) & 121.8(4) \\ C(24)-C(23)-C(22) & 121.6(4) \\ C(25)-C(24)-C(23) & 119.5(4) \\ C(24)-C(25)-C(26) & 121.7(4) \\ C(25)-C(26)-C(27) & 117.7(4) \\ C(25)-C(26)-C(31) & 119.1(4) \\ C(27)-C(26)-C(31) & 123.1(4) \\ C(22)-C(27)-C(26) & 120.6(4) \\ C(22)-C(27)-N(3) & 121.8(3) \\ C(26)-C(27)-C(3) & 117.5(3) \\ C(29)-C(28)-C(30) & 109.6(4) \\ C(29)-C(28)-C(22) & 111.3(4) \\ C(30)-C(28)-C(22) & 111.6(4) \\ C(33)-C(31)-C(26) & 112.3(5) \\ C(33)-C(31)-C(32) & 109.4(4) \\ C(26)-C(31)-C(32) & 112.1(4) \\ C(34)-O(1)-C(37) & 109.6(3) \\ C(34)-O(1)-C r(1) & 118.1(2) \\ C(37)-O(1)-C r(1) & 132.2(2) \\ O(1)-C(34)-C\left(35^{\prime}\right) & 106.6(9) \\ O(1)-C(34)-C(35) & 105.8(4) \\ C\left(35^{\prime}\right)-C(34)-C(35) & 35.6(9) \\ C(37)-C(36)-C(35) & 102.4(11) \\ C(34)-C(35)-C(36) & 105.8(9) \\ C(34)-C\left(35^{\prime}\right)-C\left(36^{\prime}\right) & 97.3(17) \\ C\left(35^{\prime}\right)-C\left(36^{\prime}\right)-C(37) & 111(2) \\ C(36)-C(37)-O(1) & 106.4(8) \\ C(36)-C(37)-C\left(36^{\prime}\right) & 3(2) \\ O(1)-C(37)-C\left(36^{\prime}\right) & 105.0(14) \\ C(38)-C(39)-O(2) & 127.2(17) \\ C(40)-0(2)-C(39) & 118.1(13) \\ O(2)-C(40)-C(41) & 120.2(14) \\ & \end{array}$

Symmetry transformations used to generate equivalent atoms: $\# 1-x+1, y,-z+3 / 2$ 
Table S10. Anisotropic displacement parameters $\left(A^{\wedge} 2 \times 10 \wedge 3\right)$ for 5. The anisotropic displacement factor exponent takes the form: $-2 p^{\wedge} 2\left[h^{\wedge} 2 a^{*} \wedge 2 \mathrm{U} 11+\ldots+2 h k a^{*} b^{*} \mathrm{U} 12\right]$

\begin{tabular}{|c|c|c|c|c|c|c|}
\hline & U11 & U22 & U33 & U23 & U13 & U12 \\
\hline $\operatorname{cr}(1)$ & $30(1)$ & $32(1)$ & $31(1)$ & $-1(1)$ & $12(1)$ & $\odot(1)$ \\
\hline$N(1)$ & $26(2)$ & $29(2)$ & $34(2)$ & $-1(1)$ & $12(1)$ & $\Theta(1)$ \\
\hline$N(2)$ & $32(2)$ & $26(2)$ & $36(2)$ & $-1(1)$ & $14(2)$ & $-1(1)$ \\
\hline$N(3)$ & $34(2)$ & $32(2)$ & $29(2)$ & $5(1)$ & $13(1)$ & $1(1)$ \\
\hline$N(4)$ & $30(2)$ & $27(2)$ & $28(2)$ & $-1(1)$ & $13(1)$ & $-1(1)$ \\
\hline$c(1)$ & $46(2)$ & $48(3)$ & $64(3)$ & $-6(2)$ & $35(2)$ & $-4(2)$ \\
\hline$C(2)$ & $31(2)$ & $38(2)$ & $35(2)$ & $2(2)$ & $17(2)$ & $3(2)$ \\
\hline$C(3)$ & $32(2)$ & $34(2)$ & $32(2)$ & $-2(2)$ & $14(2)$ & $5(2)$ \\
\hline$c(4)$ & $43(2)$ & $42(2)$ & $44(3)$ & $1(2)$ & $20(2)$ & $10(2)$ \\
\hline$C(5)$ & $56(3)$ & $33(2)$ & $54(3)$ & $-6(2)$ & $27(2)$ & $10(2)$ \\
\hline$c(6)$ & $56(3)$ & $27(2)$ & $50(3)$ & $-2(2)$ & $18(2)$ & $-1(2)$ \\
\hline$c(7)$ & $38(2)$ & $24(2)$ & $33(2)$ & $-2(2)$ & $9(2)$ & $2(2)$ \\
\hline$C(8)$ & $35(2)$ & $30(2)$ & $36(2)$ & $3(2)$ & $10(2)$ & $\odot(2)$ \\
\hline$C(9)$ & $55(3)$ & $41(2)$ & $50(3)$ & $4(2)$ & $20(2)$ & $-7(2)$ \\
\hline$C(10)$ & $37(2)$ & $33(2)$ & $44(2)$ & $-3(2)$ & $22(2)$ & $-7(2)$ \\
\hline$C(11)$ & $50(3)$ & $37(2)$ & $56(3)$ & $-2(2)$ & $26(2)$ & $-7(2)$ \\
\hline$C(12)$ & $56(3)$ & $45(3)$ & $59(3)$ & $-12(2)$ & $23(3)$ & $-21(2)$ \\
\hline$C(13)$ & $46(3)$ & $59(3)$ & $47(3)$ & $-10(2)$ & $11(2)$ & $-15(2)$ \\
\hline$C(14)$ & $30(2)$ & $46(2)$ & $45(3)$ & $-6(2)$ & $13(2)$ & $-7(2)$ \\
\hline$C(15)$ & $31(2)$ & $35(2)$ & $42(2)$ & $-7(2)$ & $21(2)$ & $-8(2)$ \\
\hline$C(16)$ & $54(3)$ & $33(2)$ & $46(3)$ & $5(2)$ & $13(2)$ & $-9(2)$ \\
\hline$c(17)$ & $92(4)$ & $95(4)$ & $53(3)$ & $18(3)$ & $33(3)$ & $-1(3)$ \\
\hline$c(18)$ & $47(3)$ & $78(4)$ & $76(4)$ & $5(3)$ & $10(3)$ & $-5(3)$ \\
\hline$C(19)$ & $41(2)$ & $54(3)$ & $54(3)$ & $6(2)$ & $2(2)$ & $-7(2)$ \\
\hline$C(20)$ & $68(4)$ & $110(5)$ & $96(5)$ & $17(4)$ & $16(4)$ & $44(4)$ \\
\hline$c(21)$ & $76(4)$ & $85(4)$ & $57(4)$ & $11(3)$ & $-4(3)$ & $-15(3)$ \\
\hline$C(22)$ & $41(2)$ & $46(2)$ & $37(2)$ & $3(2)$ & $18(2)$ & $-4(2)$ \\
\hline$C(23)$ & $46(3)$ & $74(3)$ & $39(3)$ & $1(2)$ & $21(2)$ & $-6(2)$ \\
\hline$C(24)$ & $61(3)$ & $81(4)$ & $34(3)$ & $3(2)$ & $19(2)$ & $-14(3)$ \\
\hline$C(25)$ & $48(3)$ & $63(3)$ & $38(3)$ & $14(2)$ & $8(2)$ & $-2(2)$ \\
\hline$C(26)$ & $43(2)$ & $39(2)$ & $41(3)$ & $6(2)$ & $14(2)$ & $-2(2)$ \\
\hline$C(27)$ & $37(2)$ & $32(2)$ & $33(2)$ & $3(2)$ & $13(2)$ & $-5(2)$ \\
\hline$C(28)$ & $41(2)$ & $75(3)$ & $35(2)$ & $5(2)$ & $19(2)$ & $6(2)$ \\
\hline$C(29)$ & $60(3)$ & $87(4)$ & $85(4)$ & $35(3)$ & $35(3)$ & 21(3) \\
\hline$C(30)$ & $44(3)$ & $98(4)$ & $51(3)$ & $7(3)$ & $15(2)$ & $-10(3)$ \\
\hline$C(31)$ & $49(3)$ & $70(3)$ & $45(3)$ & $6(2)$ & $9(2)$ & 18(2) \\
\hline$C(32)$ & $46(3)$ & $76(4)$ & $94(4)$ & $\odot(3)$ & $25(3)$ & $3(3)$ \\
\hline$C(33)$ & $64(4)$ & $68(4)$ & $142(7)$ & $-23(4)$ & $19(4)$ & $15(3)$ \\
\hline $0(1)$ & $46(2)$ & $37(2)$ & $33(2)$ & $-5(1)$ & $15(1)$ & $4(1)$ \\
\hline$c(34)$ & $68(3)$ & $39(2)$ & $53(3)$ & $2(2)$ & $27(2)$ & $12(2)$ \\
\hline$C(36)$ & $99(10)$ & $26(6)$ & $56(13)$ & $-13(6)$ & $23(9)$ & $11(6)$ \\
\hline$C(35)$ & $76(6)$ & $56(5)$ & $67(5)$ & $-14(4)$ & $30(5)$ & $9(5)$ \\
\hline$C\left(35^{\prime}\right)$ & $56(13)$ & $47(12)$ & $96(19)$ & $-24(11)$ & $10(14)$ & $7(11)$ \\
\hline$C\left(36^{\prime}\right)$ & $290(40)$ & $190(30)$ & $17(12)$ & $-44(16)$ & $-13(15)$ & $9 \odot(30)$ \\
\hline$C(37)$ & $67(3)$ & $50(3)$ & $29(3)$ & $-5(2)$ & $9(2)$ & $0(2)$ \\
\hline
\end{tabular}


Table S11. Hydrogen coordinates $(x 10 \wedge 4)$ and isotropic displacement parameters $\left(A^{\wedge} 2 \times 10^{\wedge} 3\right)$ for 5 .

\begin{tabular}{|c|c|c|c|c|}
\hline & $x$ & $y$ & $z$ & $U(e q)$ \\
\hline$H(1 A)$ & 7291 & 7819 & 7578 & 73 \\
\hline$H(1 B)$ & 6984 & 6971 & 7140 & 73 \\
\hline$H(1 C)$ & 7515 & 6532 & 7598 & 73 \\
\hline$H(4 A)$ & 6934 & 4697 & 7270 & 51 \\
\hline$H(5 A)$ & 6484 & $29 \odot 2$ & 7225 & 55 \\
\hline$H(6 A)$ & 5872 & 2632 & 7624 & 54 \\
\hline$H(9 A)$ & 5243 & 3301 & 8563 & 73 \\
\hline$H(9 B)$ & 5658 & 2506 & 8414 & 73 \\
\hline$H(9 C)$ & 5061 & 2927 & 8019 & 73 \\
\hline$H(11 A)$ & 6756 & 11129 & 7808 & 55 \\
\hline$H(12 A)$ & 7506 & 11330 & 8550 & 64 \\
\hline$H(13 A)$ & 7876 & 9718 & 9021 & 63 \\
\hline$H(16 A)$ & 6068 & 8478 & 7273 & 55 \\
\hline$H(17 A)$ & 6721 & 9314 & 6972 & 118 \\
\hline$H(17 B)$ & 6469 & 10557 & 6996 & 118 \\
\hline $\mathrm{H}(17 \mathrm{C})$ & 6075 & 9620 & 6633 & 118 \\
\hline$H(18 A)$ & 5501 & 9669 & 7542 & 107 \\
\hline$H(18 B)$ & 5332 & 9837 & 6983 & 107 \\
\hline$H(18 C)$ & 5719 & 10773 & 7351 & 107 \\
\hline$H(19 A)$ & 7256 & 6957 & 8855 & 66 \\
\hline$H(2 \odot A)$ & 7830 & 6670 & 8408 & 145 \\
\hline$H(20 B)$ & 8132 & 6154 & 8933 & 145 \\
\hline$H(20 C)$ & 8344 & 7349 & 8805 & 145 \\
\hline$H(21 A)$ & 7592 & 8207 & 9531 & 123 \\
\hline$H(21 B)$ & 8200 & 8293 & 9490 & 123 \\
\hline$H(21 C)$ & 7991 & 7098 & 9622 & 123 \\
\hline$H(23 A)$ & 4989 & 5754 & 9592 & 62 \\
\hline$H(24 A)$ & 5839 & 5206 & 10211 & 70 \\
\hline$H(25 A)$ & 6589 & 4483 & 10042 & 63 \\
\hline$H(28 A)$ & 4656 & 5448 & 8344 & 58 \\
\hline$H(29 A)$ & 4916 & 7391 & 8530 & 113 \\
\hline$H(29 B)$ & 4586 & 7406 & 8882 & 113 \\
\hline$H(29 C)$ & 4235 & 7246 & 8322 & 113 \\
\hline$H(3 \odot A)$ & 3797 & 5481 & 8482 & 98 \\
\hline$H(30 B)$ & 4162 & 5562 & 9043 & 98 \\
\hline$H(30 \mathrm{C})$ & 4191 & 4429 & 8759 & 98 \\
\hline$H(31 A)$ & 6686 & 4486 & 8895 & 69 \\
\hline$H(32 A)$ & 7262 & 5701 & 9529 & 109 \\
\hline$H(32 B)$ & 7622 & 4625 & 9476 & 109 \\
\hline$H(32 C)$ & 7389 & 4651 & 9893 & 109 \\
\hline$H(33 A)$ & 6464 & 2635 & 9065 & 147 \\
\hline$H(33 B)$ & 6898 & 2785 & 9608 & 147 \\
\hline$H(33 C)$ & 7135 & 2769 & 9194 & 147 \\
\hline$H(34 A)$ & 5507 & 9116 & 8305 & 63 \\
\hline$H(34 B)$ & 6168 & 9502 & 8522 & 63 \\
\hline $\mathrm{H}(34 \mathrm{C})$ & 5449 & 9159 & 8473 & 63 \\
\hline$H(34 D)$ & 5994 & 9343 & 8337 & 63 \\
\hline$H(36 A)$ & 6594 & 9372 & 9564 & 76 \\
\hline
\end{tabular}




\begin{tabular}{lrrrr}
$H(36 B)$ & 6074 & 8934 & $970 \odot$ & 76 \\
$H(35 A)$ & 5398 & 9479 & 8987 & 79 \\
$H(35 B)$ & 5875 & 10428 & 9030 & 79 \\
$H(35 C)$ & 5919 & 10451 & 9056 & 87 \\
$H(35 D)$ & 6541 & 10072 & 9074 & 87 \\
$H(36 C)$ & 5840 & 8855 & 9470 & 228 \\
$H(36 D)$ & 6522 & 8995 & 9708 & 228 \\
$H(37 A)$ & 6019 & 7210 & 9324 & 62 \\
$H(37 B)$ & 6657 & 7493 & 9367 & 62 \\
$H(37 C)$ & 6663 & 7530 & 9370 & 62 \\
$H(37 D)$ & 6030 & 7188 & 9325 & 62 \\
$H(38 A)$ & 6870 & 1394 & 914 & 166 \\
$H(38 B)$ & 6362 & 1744 & 1073 & 166 \\
$H(38 C)$ & 6597 & 2648 & 799 & 166 \\
$H(39 A)$ & 6113 & 666 & 488 & 138 \\
$H(39 B)$ & 6356 & 1538 & 217 & 138 \\
$H(40 A)$ & 5275 & 707 & -159 & 108 \\
$H(40 B)$ & 5632 & 1505 & -364 & 108 \\
$H(41 A)$ & 4674 & 1672 & -813 & 222 \\
$H(41 B)$ & 4919 & 2875 & -567 & 222 \\
$H(41 C)$ & 4561 & 2081 & -357 & 222 \\
\hline
\end{tabular}


Table S12. Torsion angles [deg] for 5.

\begin{tabular}{|c|c|}
\hline$N(4)-C r(1)-N(1)-C(2)$ & $-88.0(3)$ \\
\hline$N(2)-C r(1)-N(1)-C(2)$ & $11.3(3)$ \\
\hline$N(3)-C r(1)-N(1)-C(2)$ & $61.1(3)$ \\
\hline$O(1)-C r(1)-N(1)-C(2)$ & $166.0(3)$ \\
\hline$N(4)-C r(1)-N(1)-C(15)$ & $86.7(3)$ \\
\hline$N(2)-C r(1)-N(1)-C(15)$ & $-173.9(3)$ \\
\hline$N(3)-C r(1)-N(1)-C(15)$ & $-124.2(3)$ \\
\hline $\mathrm{O}(1)-\mathrm{Cr}(1)-\mathrm{N}(1)-\mathrm{C}(15)$ & $-19.3(3)$ \\
\hline$N(4)-C r(1)-N(2)-C(3)$ & $97.1(3)$ \\
\hline$N(3)-C r(1)-N(2)-C(3)$ & $-158.2(3)$ \\
\hline$N(1)-C r(1)-N(2)-C(3)$ & $-9.9(3)$ \\
\hline $\mathrm{O}(1)-\mathrm{Cr}(1)-\mathrm{N}(2)-\mathrm{C}(3)$ & $-75.1(4)$ \\
\hline$N(4)-C r(1)-N(2)-C(7)$ & $-85.9(3)$ \\
\hline$N(3)-C r(1)-N(2)-C(7)$ & $18.8(3)$ \\
\hline$N(1)-C r(1)-N(2)-C(7)$ & $167.1(3)$ \\
\hline$O(1)-\operatorname{Cr}(1)-N(2)-C(7)$ & $101.9(3)$ \\
\hline$N(4)-C r(1)-N(3)-C(8)$ & $80.3(3)$ \\
\hline$N(2)-C r(1)-N(3)-C(8)$ & $-19.9(2)$ \\
\hline$N(1)-C r(1)-N(3)-C(8)$ & $-69.3(3)$ \\
\hline $\mathrm{O}(1)-\mathrm{Cr}(1)-\mathrm{N}(3)-\mathrm{C}(8)$ & $-171.7(2)$ \\
\hline$N(4)-C r(1)-N(3)-C(27)$ & $-109.5(3)$ \\
\hline$N(2)-C r(1)-N(3)-C(27)$ & $150.3(3)$ \\
\hline$N(1)-C r(1)-N(3)-C(27)$ & $101.0(3)$ \\
\hline$O(1)-\operatorname{Cr}(1)-N(3)-C(27)$ & $-1.5(3)$ \\
\hline$N(2)-C r(1)-N(4)-N(4) \# 1$ & $19.1(10)$ \\
\hline$N(3)-\operatorname{Cr}(1)-N(4)-N(4) \# 1$ & $-60.8(10)$ \\
\hline$N(1)-\operatorname{Cr}(1)-N(4)-N(4) \# 1$ & $98.0(10)$ \\
\hline $\mathrm{O}(1)-\mathrm{Cr}(1)-\mathrm{N}(4)-\mathrm{N}(4) \# 1$ & $-164.7(10)$ \\
\hline$C(15)-N(1)-C(2)-C(3)$ & $173.6(3)$ \\
\hline$C r(1)-N(1)-C(2)-C(3)$ & $-11.3(4)$ \\
\hline$C(15)-N(1)-C(2)-C(1)$ & $-6.9(6)$ \\
\hline$C r(1)-N(1)-C(2)-C(1)$ & $168.2(3)$ \\
\hline$C(7)-N(2)-C(3)-C(4)$ & $6.7(5)$ \\
\hline$C r(1)-N(2)-C(3)-C(4)$ & $-176.4(3)$ \\
\hline$C(7)-N(2)-C(3)-C(2)$ & $-169.9(3)$ \\
\hline$C r(1)-N(2)-C(3)-C(2)$ & $7.0(4)$ \\
\hline$N(1)-C(2)-C(3)-N(2)$ & $3.6(5)$ \\
\hline$C(1)-C(2)-C(3)-N(2)$ & $-175.9(4)$ \\
\hline$N(1)-C(2)-C(3)-C(4)$ & $-172.7(4)$ \\
\hline$C(1)-C(2)-C(3)-C(4)$ & $7.8(6)$ \\
\hline$N(2)-C(3)-C(4)-C(5)$ & $0.3(6)$ \\
\hline$C(2)-c(3)-c(4)-C(5)$ & $176.3(4)$ \\
\hline$C(3)-C(4)-C(5)-C(6)$ & $-3.9(7)$ \\
\hline$C(4)-C(5)-C(6)-C(7)$ & $0.6(7)$ \\
\hline$C(5)-C(6)-C(7)-N(2)$ & $6.3(6)$ \\
\hline$C(5)-C(6)-C(7)-C(8)$ & $-164.4(4)$ \\
\hline$C(3)-N(2)-C(7)-C(6)$ & $-9.9(5)$ \\
\hline $\operatorname{Cr}(1)-N(2)-C(7)-C(6)$ & $173.1(3)$ \\
\hline$C(3)-N(2)-C(7)-C(8)$ & $162.3(3)$ \\
\hline $\operatorname{Cr}(1)-N(2)-C(7)-C(8)$ & $-14.8(4)$ \\
\hline $\mathrm{C}(27)-\mathrm{N}(3)-\mathrm{C}(8)-\mathrm{C}(7)$ & $-153.3(3)$ \\
\hline$C r(1)-N(3)-C(8)-C(7)$ & $18.1(4)$ \\
\hline$C(27)-N(3)-C(8)-C(9)$ & $27.1(5)$ \\
\hline
\end{tabular}




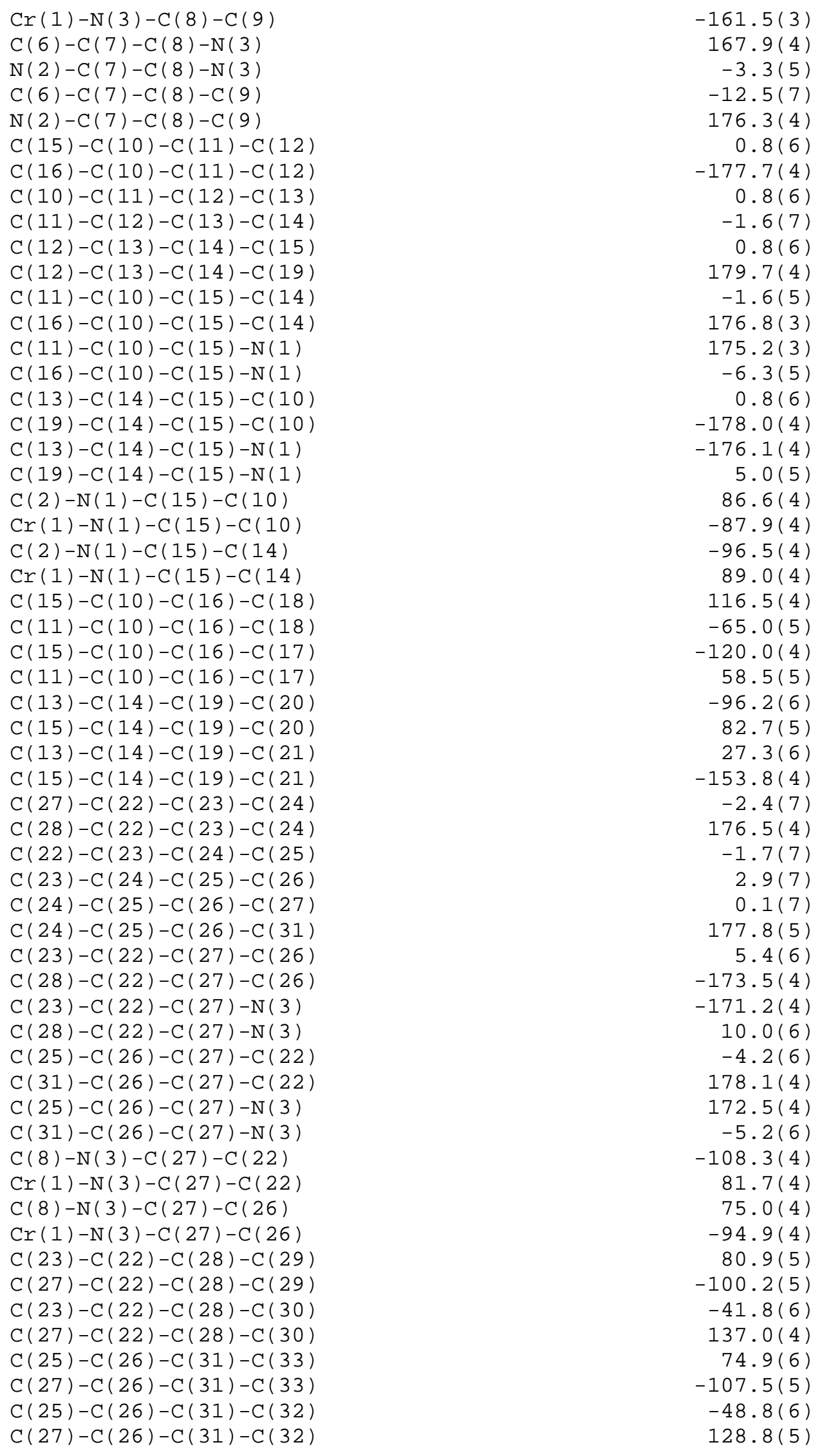




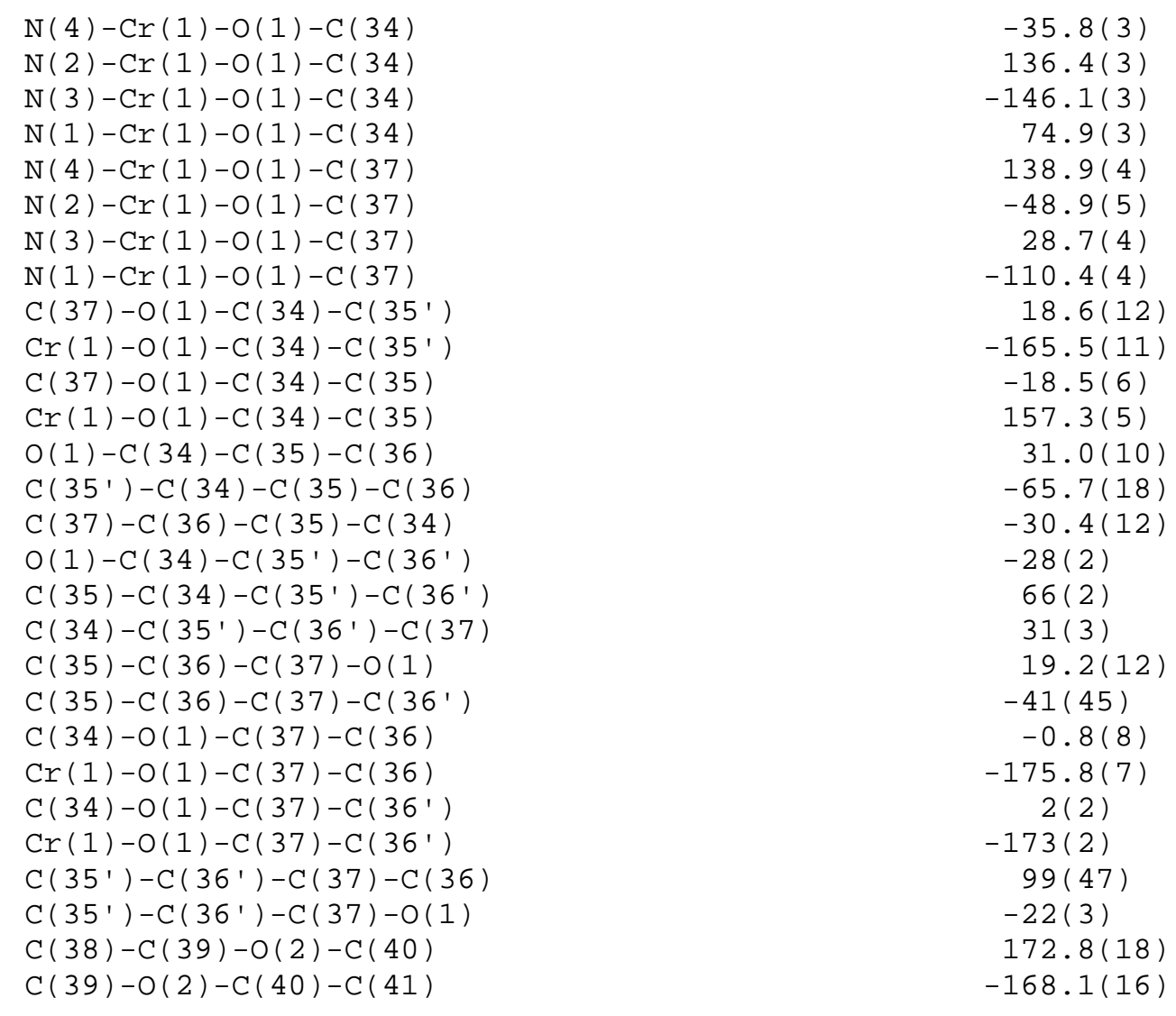

Symmetry transformations used to generate equivalent atoms: $\# 1-x+1, y,-z+3 / 2$ 
Complex 6 
Table S13. Crystal data and structure refinement for 6 .

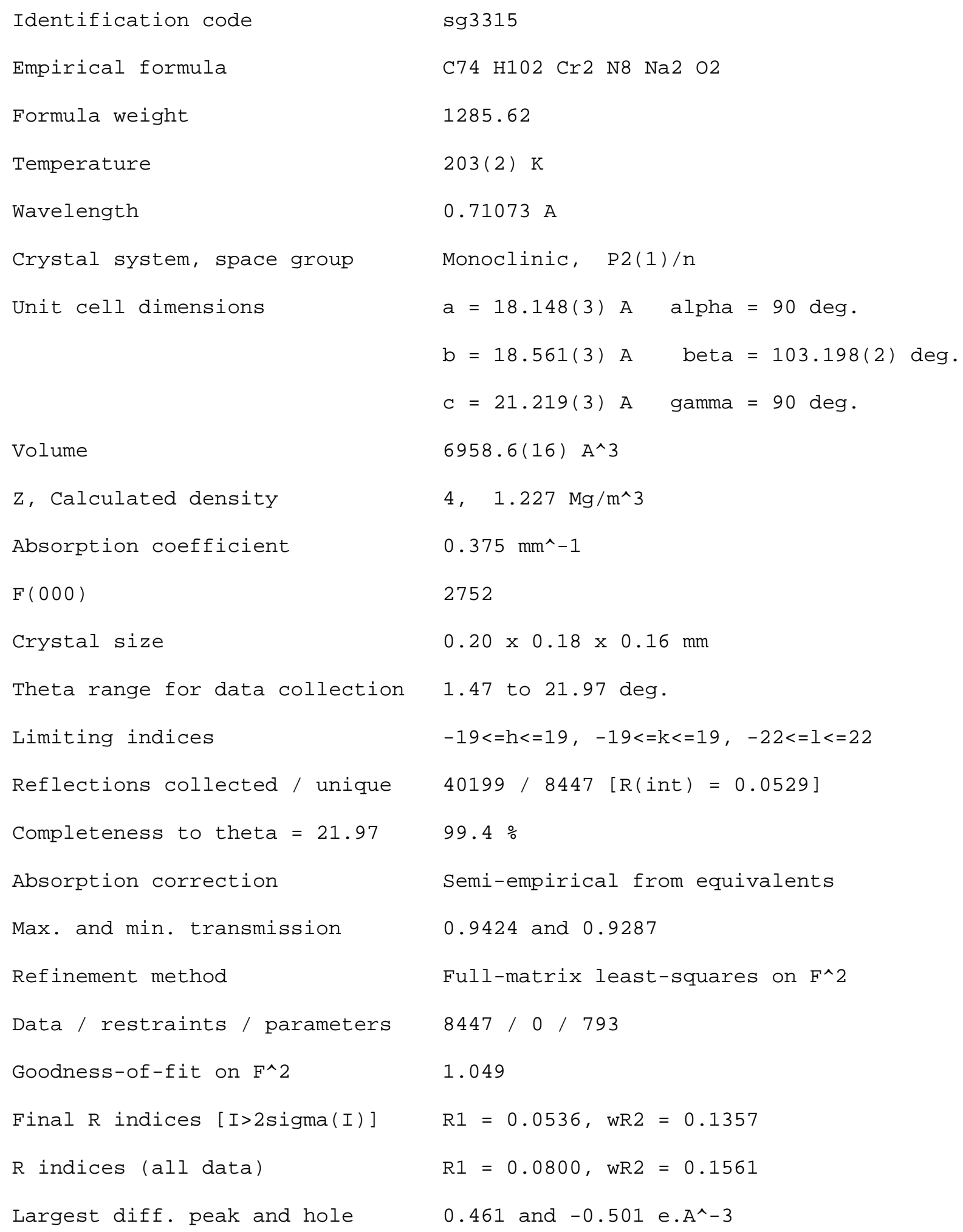


Table 14. Atomic coordinates $(x 10 \wedge 4)$ and equivalent isotropic displacement parameters $\left(A^{\wedge} 2 \times 10 \wedge 3\right)$ for 6 .

$\mathrm{U}(\mathrm{eq})$ is defined as one third of the trace of the orthogonalized Uij tensor.

\begin{tabular}{|c|c|c|c|c|}
\hline & $x$ & $y$ & $z$ & $\mathrm{U}(\mathrm{eq})$ \\
\hline $\operatorname{cr}(1)$ & $5546(1)$ & $8178(1)$ & $2095(1)$ & $30(1)$ \\
\hline $\operatorname{cr}(2)$ & $4182(1)$ & $7927(1)$ & $3711(1)$ & $28(1)$ \\
\hline $\mathrm{Na}(1)$ & $3872(1)$ & $8592(1)$ & $2310(1)$ & $47(1)$ \\
\hline $\mathrm{Na}(2)$ & $5747(1)$ & $8826(1)$ & $3465(1)$ & $49(1)$ \\
\hline$N(1)$ & $4895(2)$ & $7877(2)$ & $1210(2)$ & $31(1)$ \\
\hline$N(2)$ & $4953(2)$ & $9 \odot 47(2)$ & $1815(2)$ & $29(1)$ \\
\hline$N(3)$ & $6308(2)$ & $8993(2)$ & $2480(2)$ & $30(1)$ \\
\hline$N(4)$ & $3290(2)$ & $8612(2)$ & $3315(2)$ & $27(1)$ \\
\hline$N(5)$ & $4601(2)$ & $8868(2)$ & $3993(2)$ & $27(1)$ \\
\hline$N(6)$ & $4884(2)$ & $7703(2)$ & $4597(2)$ & $27(1)$ \\
\hline$N(7)$ & $5123(2)$ & $7860(2)$ & $2716(2)$ & $27(1)$ \\
\hline$N(8)$ & $4668(2)$ & $7756(2)$ & $3098(2)$ & $26(1)$ \\
\hline $0(1)$ & $6416(2)$ & $7414(2)$ & $2092(1)$ & $34(1)$ \\
\hline $0(2)$ & $3456(2)$ & $7011(2)$ & $37 \odot \odot(2)$ & $34(1)$ \\
\hline$C(1)$ & $3653(3)$ & $8156(3)$ & $439(2)$ & $45(1)$ \\
\hline$C(2)$ & $4295(2)$ & $8347(3)$ & $968(2)$ & $33(1)$ \\
\hline$C(3)$ & $4340(3)$ & $9014(3)$ & $1289(2)$ & $33(1)$ \\
\hline$C(4)$ & $3866(3)$ & $9626(3)$ & $1146(2)$ & $44(1)$ \\
\hline$C(5)$ & $4029(3)$ & $10241(3)$ & $1489(3)$ & $48(1)$ \\
\hline
\end{tabular}




\begin{tabular}{|c|c|c|c|c|}
\hline$C(6)$ & $4698(3)$ & $10325(3)$ & $1999(3)$ & $44(1)$ \\
\hline$c(7)$ & $5204(3)$ & $9696(2)$ & $2121(2)$ & $31(1)$ \\
\hline$C(8)$ & $5938(3)$ & $9670(3)$ & $2487(2)$ & $34(1)$ \\
\hline$C(9)$ & $6315(3)$ & $10272(3)$ & $2882(3)$ & $48(1)$ \\
\hline$c(10)$ & $4933(2)$ & 7246 (3) & $819(2)$ & $32(1)$ \\
\hline$c(11)$ & $5183(3)$ & $7318(3)$ & $236(2)$ & $38(1)$ \\
\hline$c(12)$ & $5140(3)$ & $6713(3)$ & $-157(2)$ & $48(1)$ \\
\hline$c(13)$ & $49 \odot 4(3)$ & $6063(3)$ & $8(3)$ & $57(2)$ \\
\hline$C(14)$ & $47 \odot 2(3)$ & $5989(3)$ & $597(3)$ & $50(1)$ \\
\hline$c(15)$ & $4720(3)$ & $6571(3)$ & $1013(2)$ & $36(1)$ \\
\hline$C(16)$ & $5503(3)$ & $8017(3)$ & $31(3)$ & $46(1)$ \\
\hline$c(17)$ & $4952(4)$ & $8406(5)$ & $-5 \odot 2(4)$ & $107(3)$ \\
\hline$c(18)$ & $6230(3)$ & $7890(3)$ & $-196(3)$ & $54(2)$ \\
\hline$c(19)$ & $4474(3)$ & $6463(3)$ & $1645(2)$ & $39(1)$ \\
\hline$c(20)$ & $3659(3)$ & $6689(3)$ & $1574(3)$ & $58(2)$ \\
\hline$C(21)$ & $4596(3)$ & $5708(3)$ & $1924(3)$ & $54(2)$ \\
\hline$c(22)$ & $7095(2)$ & $9 \odot 42(2)$ & $2470(2)$ & $28(1)$ \\
\hline$c(23)$ & $7313(3)$ & $9406(2)$ & $1955(2)$ & $34(1)$ \\
\hline$c(24)$ & $8081(3)$ & $9473(3)$ & $1973(2)$ & $41(1)$ \\
\hline$c(25)$ & $8627(3)$ & $9195(3)$ & 2466 (3) & $48(1)$ \\
\hline$C(26)$ & $8411(3)$ & $8816(3)$ & 2948 (3) & $46(1)$ \\
\hline$c(27)$ & $7654(3)$ & $8729(3)$ & $2961(2)$ & $35(1)$ \\
\hline$c(28)$ & $6753(3)$ & $9698(3)$ & $1371(2)$ & $43(1)$ \\
\hline$C(29)$ & $6728(4)$ & $10520(3)$ & $1349(3)$ & $69(2)$ \\
\hline$c(30)$ & $6915(3)$ & $9419(3)$ & $74 \odot(3)$ & $59(2)$ \\
\hline$c(31)$ & $7460(3)$ & $8312(3)$ & $3520(2)$ & $45(1)$ \\
\hline$c(32)$ & $7428(4)$ & $8821(4)$ & $4082(3)$ & $75(2)$ \\
\hline$c(33)$ & $80 \odot 9(3)$ & $7693(4)$ & $3758(3)$ & $72(2)$ \\
\hline
\end{tabular}




\begin{tabular}{|c|c|c|c|c|}
\hline$c(34)$ & $6896(3)$ & $7394(3)$ & $1632(2)$ & $39(1)$ \\
\hline$c(35)$ & $7171(5)$ & $6649(3)$ & $1630(4)$ & $97(3)$ \\
\hline$C(36)$ & $6760(4)$ & 6218 (3) & $1995(4)$ & $88(2)$ \\
\hline$c(37)$ & $6475(3)$ & $6732(3)$ & $2425(3)$ & $46(1)$ \\
\hline$c(38)$ & $3052(3)$ & $9881(3)$ & $2882(3)$ & $45(1)$ \\
\hline$C(39)$ & $3532(2)$ & $9333(2)$ & $3293(2)$ & $30(1)$ \\
\hline$c(4 \odot)$ & $4253(2)$ & $9479(2)$ & $3662(2)$ & $29(1)$ \\
\hline$C(41)$ & $4658(3)$ & $10160(3)$ & $3759(2)$ & $37(1)$ \\
\hline$C(42)$ & $5341(3)$ & $10174(3)$ & $4249(2)$ & $38(1)$ \\
\hline$C(43)$ & $5603(3)$ & $9583(2)$ & $4621(2)$ & $33(1)$ \\
\hline$C(44)$ & $5225(2)$ & $8920(2)$ & $4504(2)$ & $28(1)$ \\
\hline$c(45)$ & $5398(2)$ & $8249(2)$ & $4836(2)$ & $29(1)$ \\
\hline$C(46)$ & $6 \odot 41(3)$ & $8167(3)$ & $5365(2)$ & $45(1)$ \\
\hline$C(47)$ & $2495(2)$ & $8535(2)$ & $3292(2)$ & $29(1)$ \\
\hline$C(48)$ & $2018(2)$ & $8140(3)$ & $2797(2)$ & $34(1)$ \\
\hline$C(49)$ & $1249(3)$ & $8112(3)$ & $2769(2)$ & $46(1)$ \\
\hline$C(50)$ & $936(3)$ & $8459(3)$ & $3217(2)$ & $46(1)$ \\
\hline$C(51)$ & $1402(3)$ & $8826(3)$ & $3714(2)$ & $38(1)$ \\
\hline$C(52)$ & $2182(2)$ & $8862(2)$ & $3774(2)$ & $30(1)$ \\
\hline$c(53)$ & $2320(3)$ & $7761(3)$ & $2268(2)$ & $41(1)$ \\
\hline$C(54)$ & $2211(3)$ & $8241(3)$ & $1669(3)$ & $6 \odot(2)$ \\
\hline$C(55)$ & $1963(3)$ & $7024(3)$ & $2100(3)$ & $61(2)$ \\
\hline$C(56)$ & $2651(3)$ & $9247(3)$ & $4359(2)$ & $35(1)$ \\
\hline$C(57)$ & $2437(3)$ & $10 \odot 45(3)$ & $4372(3)$ & $57(2)$ \\
\hline$C(58)$ & $2568(3)$ & $8887(3)$ & $4987(2)$ & $53(2)$ \\
\hline$C(59)$ & $4990(2)$ & $7040(2)$ & $4961(2)$ & $27(1)$ \\
\hline$C(60)$ & $4664(2)$ & $6962(2)$ & $5501(2)$ & $28(1)$ \\
\hline$C(61)$ & $4799(3)$ & $6330(3)$ & $5861(2)$ & $41(1)$ \\
\hline$c(62)$ & $5235(3)$ & $5785(3)$ & $5704(2)$ & $46(1)$ \\
\hline
\end{tabular}




\begin{tabular}{lllll}
$C(63)$ & $5536(3)$ & $5860(3)$ & $5169(2)$ & $42(1)$ \\
$C(64)$ & $5431(3)$ & $6487(2)$ & $4792(2)$ & $32(1)$ \\
$C(65)$ & $4186(3)$ & $7542(3)$ & $5716(2)$ & $36(1)$ \\
$C(66)$ & $3462(3)$ & $7237(3)$ & $5871(3)$ & $47(1)$ \\
$C(67)$ & $4625(3)$ & $7944(3)$ & $6313(3)$ & $51(1)$ \\
$C(68)$ & $5838(3)$ & $6547(3)$ & $4248(3)$ & $50(2)$ \\
$C(69)$ & $5744(3)$ & $5896(3)$ & $3803(3)$ & $64(2)$ \\
$C(70)$ & $6678(4)$ & $6648(5)$ & $4516(4)$ & $129(4)$ \\
$C(71)$ & $2926(3)$ & $6925(3)$ & $4115(3)$ & $42(1)$ \\
$C(72)$ & $2976(3)$ & $6139(3)$ & $4306(3)$ & $54(2)$ \\
$C(73)$ & $3164(4)$ & $5774(3)$ & $3728(3)$ & $64(2)$ \\
$C(74)$ & $3640(3)$ & $6305(3)$ & $3493(3)$ & $55(2)$ \\
\hline
\end{tabular}


Table S15. Bond lengths [A] and angles [deg] for 6 .

\begin{tabular}{|c|c|}
\hline $\operatorname{Cr}(1)-\mathrm{N}(7)$ & $1.771(4)$ \\
\hline $\operatorname{Cr}(1)-N(2)$ & $1.953(4)$ \\
\hline $\operatorname{Cr}(1)-\mathrm{N}(1)$ & $2.054(4)$ \\
\hline $\operatorname{Cr}(1)-\mathrm{N}(3)$ & $2.086(4)$ \\
\hline $\operatorname{Cr}(1)-0(1)$ & $2.125(3)$ \\
\hline $\mathrm{Cr}(1)-\mathrm{Na}(2)$ & $3.089(2)$ \\
\hline $\mathrm{Cr}(1)-\mathrm{Na}(1)$ & $3.264(2)$ \\
\hline $\operatorname{Cr}(2)-\mathrm{N}(8)$ & $1.760(3)$ \\
\hline $\operatorname{Cr}(2)-N(5)$ & $1.945(4)$ \\
\hline $\operatorname{Cr}(2)-N(6)$ & $2.059(3)$ \\
\hline $\operatorname{Cr}(2)-N(4)$ & $2.079(3)$ \\
\hline $\operatorname{cr}(2)-0(2)$ & $2.147(3)$ \\
\hline $\mathrm{Cr}(2)-\mathrm{Na}(1)$ & $3.1472(19)$ \\
\hline $\mathrm{Cr}(2)-\mathrm{Na}(2)$ & $3.435(2)$ \\
\hline $\mathrm{Na}(1)-\mathrm{N}(8)$ & $2.486(4)$ \\
\hline $\mathrm{Na}(1)-\mathrm{N}(2)$ & $2.571(4)$ \\
\hline $\mathrm{Na}(1)-\mathrm{N}(4)$ & $2.587(4)$ \\
\hline $\mathrm{Na}(1)-\mathrm{N}(7)$ & $2.617(4)$ \\
\hline $\mathrm{Na}(1)-\mathrm{C}(3)$ & $2.621(5)$ \\
\hline $\mathrm{Na}(1)-\mathrm{C}(39)$ & $2.685(5)$ \\
\hline $\mathrm{Na}(1)-\mathrm{C}(54)$ & $3.080(6)$ \\
\hline $\mathrm{Na}(1)-\mathrm{C}(4)$ & $3.127(5)$ \\
\hline $\mathrm{Na}(1)-\mathrm{Na}(2)$ & $3.736(3)$ \\
\hline $\mathrm{Na}(2)-\mathrm{N}(7)$ & $2.488(4)$ \\
\hline $\mathrm{Na}(2)-\mathrm{N}(3)$ & $2.544(4)$ \\
\hline
\end{tabular}




$$
\begin{aligned}
& \mathrm{Na}(2)-\mathrm{N}(5) \quad 2.578(4) \\
& \mathrm{Na}(2)-\mathrm{C}(44) \quad 2.597(5) \\
& \mathrm{Na}(2)-\mathrm{C}(8) \quad 2.686(5) \\
& \mathrm{Na}(2)-\mathrm{N}(8) \quad 2.772(4) \\
& \mathrm{Na}(2)-\mathrm{C}(43) \quad 2.891(5) \\
& \mathrm{Na}(2)-\mathrm{C}(32) \quad 3.033(7) \\
& \mathrm{Na}(2)-\mathrm{C}(40) \quad 3.086(5) \\
& N(1)-C(2) \quad 1.399(6) \\
& N(1)-C(10) \quad 1.445(6) \\
& N(2)-C(3) \quad 1.386(6) \\
& \mathrm{N}(2)-\mathrm{C}(7) \quad 1.395(6) \\
& \mathrm{N}(3)-\mathrm{C}(8) \quad 1.426(6) \\
& \mathrm{N}(3)-\mathrm{C}(22) \quad 1.436(5) \\
& \mathrm{N}(4)-\mathrm{C}(39) \quad 1.413(6) \\
& N(4)-C(47) \quad 1.438(5) \\
& N(5)-C(44) \quad 1.381(5) \\
& N(5)-C(40) \quad 1.407(6) \\
& N(6)-C(45) \quad 1.393(6) \\
& N(6)-C(59) \quad 1.442(5) \\
& \mathrm{N}(7)-\mathrm{N}(8) \quad 1.296(5) \\
& 0(1)-C(37) \quad 1.440(6) \\
& 0(1)-C(34) \quad 1.450(5) \\
& O(2)-C(74) \quad 1.444(6) \\
& 0(2)-C(71) \quad 1.454(5) \\
& \mathrm{C}(1)-\mathrm{C}(2) \quad 1.466(6) \\
& \mathrm{C}(2)-\mathrm{C}(3) \quad 1.405(7) \\
& \mathrm{C}(3)-\mathrm{C}(4) \quad 1.416(7) \\
& \mathrm{C}(4)-\mathrm{C}(5) \quad 1.350(7)
\end{aligned}
$$




\begin{tabular}{|c|c|}
\hline$C(5)-C(6)$ & $1.438(7)$ \\
\hline$C(6)-C(7)$ & $1.471(7)$ \\
\hline$C(7)-C(8)$ & $1.382(6)$ \\
\hline$C(8)-C(9)$ & $1.470(7)$ \\
\hline$C(10)-C(15)$ & $1.400(7)$ \\
\hline$C(10)-C(11)$ & $1.417(6)$ \\
\hline$C(11)-C(12)$ & $1.391(7)$ \\
\hline$C(11)-C(16)$ & $1.524(7)$ \\
\hline$c(12)-c(13)$ & $1.352(8)$ \\
\hline$C(13)-C(14)$ & $1.388(7)$ \\
\hline$C(14)-C(15)$ & $1.392(7)$ \\
\hline$C(15)-C(19)$ & $1.521(7)$ \\
\hline$C(16)-C(17)$ & $1.512(8)$ \\
\hline$C(16)-C(18)$ & $1.524(7)$ \\
\hline$C(19)-C(21)$ & $1.518(7)$ \\
\hline$C(19)-C(20)$ & $1.512(7)$ \\
\hline$C(22)-C(27)$ & $1.405(6)$ \\
\hline$C(22)-C(23)$ & $1.416(6)$ \\
\hline$C(23)-C(24)$ & $1.390(6)$ \\
\hline$C(23)-C(28)$ & $1.513(7)$ \\
\hline$C(24)-C(25)$ & $1.368(7)$ \\
\hline$C(25)-C(26)$ & $1.371(7)$ \\
\hline$C(26)-C(27)$ & $1.389(7)$ \\
\hline$C(27)-C(31)$ & $1.523(7)$ \\
\hline$C(28)-C(30)$ & $1.525(7)$ \\
\hline$C(28)-C(29)$ & $1.525(8)$ \\
\hline$C(31)-C(33)$ & $1.529(8)$ \\
\hline$C(31)-C(32)$ & $1.532(8)$ \\
\hline$c(34)-c(35)$ & $1.470(8)$ \\
\hline
\end{tabular}




$$
\begin{aligned}
& C(35)-C(36) \quad 1.434(8) \\
& \mathrm{C}(36)-\mathrm{C}(37) \quad 1.491(8) \\
& \mathrm{C}(38)-\mathrm{C}(39) \quad 1.486(6) \\
& \mathrm{C}(39)-\mathrm{C}(40) \quad 1.389(6) \\
& \mathrm{C}(40)-\mathrm{C}(41) \quad 1.453(6) \\
& C(41)-C(42) \quad 1.425(7) \\
& \mathrm{C}(42)-\mathrm{C}(43) \quad 1.372(7) \\
& C(43)-C(44) \quad 1.402(6) \\
& \mathrm{C}(44)-\mathrm{C}(45) \quad 1.431(6) \\
& C(45)-C(46) \quad 1.430(6) \\
& \mathrm{C}(47)-\mathrm{C}(48) \quad 1.406(6) \\
& C(47)-C(52) \quad 1.413(6) \\
& \mathrm{C}(48)-\mathrm{C}(49) \quad 1.384(7) \\
& \mathrm{C}(48)-\mathrm{C}(53) \quad 1.528(7) \\
& \mathrm{C}(49)-\mathrm{C}(50) \quad 1.374(7) \\
& \mathrm{C}(50)-\mathrm{C}(51) \quad 1.373(7) \\
& C(51)-C(52) \quad 1.395(6) \\
& \mathrm{C}(52)-\mathrm{C}(56) \quad 1.515(6) \\
& C(53)-C(55) \quad 1.520(7) \\
& C(53)-C(54) \quad 1.527(7) \\
& \mathrm{C}(56)-\mathrm{C}(58) \quad 1.528(7) \\
& \mathrm{C}(56)-\mathrm{C}(57) \quad 1.533(7) \\
& \mathrm{C}(59)-\mathrm{C}(64) \quad 1.397(6) \\
& C(59)-C(60) \quad 1.412(6) \\
& C(60)-C(61) \quad 1.391(6) \\
& C(60)-C(65) \quad 1.517(6) \\
& \mathrm{C}(61)-\mathrm{C}(62) \quad 1.371(7) \\
& C(62)-C(63) \quad 1.374(7)
\end{aligned}
$$




$$
\begin{aligned}
& C(63)-C(64) \quad 1.400(7) \\
& \mathrm{C}(64)-\mathrm{C}(68) \quad 1.512(7) \\
& C(65)-C(67) \quad 1.529(7) \\
& C(65)-C(66) \quad 1.534(6) \\
& \mathrm{C}(68)-\mathrm{C}(70) \quad 1.513(9) \\
& \mathrm{C}(68)-\mathrm{C}(69) \quad 1.519(8) \\
& \mathrm{C}(71)-\mathrm{C}(72) \quad 1.511(7) \\
& \mathrm{C}(72)-\mathrm{C}(73) \quad 1.507(8) \\
& C(73)-C(74) \quad 1.470(7) \\
& N(7)-\operatorname{Cr}(1)-N(2) \quad 101.51(15) \\
& N(7)-\operatorname{Cr}(1)-N(1) \quad 109.51(15) \\
& \mathrm{N}(2)-\operatorname{Cr}(1)-\mathrm{N}(1) \quad 77.63(15) \\
& \mathrm{N}(7)-\operatorname{Cr}(1)-\mathrm{N}(3) \quad 108.09(15) \\
& \mathrm{N}(2)-\operatorname{Cr}(1)-\mathrm{N}(3) \quad 77.94(15) \\
& N(1)-\operatorname{Cr}(1)-N(3) \quad 138.34(15) \\
& N(7)-\operatorname{Cr}(1)-0(1) \quad 103.16(14) \\
& \mathrm{N}(2)-\operatorname{Cr}(1)-0(1) \quad 155.30(13) \\
& N(1)-\operatorname{Cr}(1)-0(1) \quad 95.06(13) \\
& N(3)-\operatorname{Cr}(1)-0(1) \quad 93.30(13) \\
& \mathrm{N}(7)-\mathrm{Cr}(1)-\mathrm{Na}(2) \quad 53.62(12) \\
& \mathrm{N}(2)-\mathrm{Cr}(1)-\mathrm{Na}(2) \quad 84.48(11) \\
& \mathrm{N}(1)-\mathrm{Cr}(1)-\mathrm{Na}(2) \quad 152.38(11) \\
& \mathrm{N}(3)-\mathrm{Cr}(1)-\mathrm{Na}(2) \quad 54.83(10) \\
& \mathrm{O}(1)-\mathrm{Cr}(1)-\mathrm{Na}(2) \quad 109.49(9) \\
& \mathrm{N}(7)-\mathrm{Cr}(1)-\mathrm{Na}(1) \quad 53.09(11) \\
& \mathrm{N}(2)-\mathrm{Cr}(1)-\mathrm{Na}(1) \quad 51.94(11) \\
& \mathrm{N}(1)-\mathrm{Cr}(1)-\mathrm{Na}(1) \quad 80.41(11) \\
& \mathrm{N}(3)-\mathrm{Cr}(1)-\mathrm{Na}(1) \quad 109.27(11)
\end{aligned}
$$




$$
\begin{aligned}
& 0(1)-\mathrm{Cr}(1)-\mathrm{Na}(1) \quad 150.87(9) \\
& \mathrm{Na}(2)-\mathrm{Cr}(1)-\mathrm{Na}(1) \quad 71.98(5) \\
& N(8)-\operatorname{Cr}(2)-N(5) \quad 99.32(15) \\
& N(8)-\operatorname{Cr}(2)-N(6) \quad 109.22(15) \\
& N(5)-\operatorname{Cr}(2)-N(6) \quad 77.44(14) \\
& \mathrm{N}(8)-\mathrm{Cr}(2)-\mathrm{N}(4) \quad 106.73(15) \\
& N(5)-\operatorname{Cr}(2)-N(4) \quad 77.81(14) \\
& N(6)-\operatorname{Cr}(2)-N(4) \quad 138.94(14) \\
& N(8)-C r(2)-0(2) \quad 105.06(14) \\
& N(5)-\operatorname{Cr}(2)-0(2) \quad 155.57(13) \\
& N(6)-\operatorname{Cr}(2)-0(2) \quad 95.74(13) \\
& N(4)-\operatorname{Cr}(2)-0(2) \quad 93.48(13) \\
& \mathrm{N}(8)-\mathrm{Cr}(2)-\mathrm{Na}(1) \quad 52.00(12) \\
& \mathrm{N}(5)-\mathrm{Cr}(2)-\mathrm{Na}(1) \quad 84.91(11) \\
& \mathrm{N}(6)-\mathrm{Cr}(2)-\mathrm{Na}(1) \quad 151.83(11) \\
& \mathrm{N}(4)-\mathrm{Cr}(2)-\mathrm{Na}(1) \quad 54.86(10) \\
& \mathrm{O}(2)-\mathrm{Cr}(2)-\mathrm{Na}(1) \quad 108.88(9) \\
& \mathrm{N}(8)-\mathrm{Cr}(2)-\mathrm{Na}(2) \quad 53.37(12) \\
& \mathrm{N}(5)-\mathrm{Cr}(2)-\mathrm{Na}(2) \quad 48.02(11) \\
& \mathrm{N}(6)-\mathrm{Cr}(2)-\mathrm{Na}(2) \quad 82.88(10) \\
& \mathrm{N}(4)-\mathrm{Cr}(2)-\mathrm{Na}(2) \quad 103.68(10) \\
& \mathrm{O}(2)-\mathrm{Cr}(2)-\mathrm{Na}(2) \quad 155.41(9) \\
& \mathrm{Na}(1)-\mathrm{Cr}(2)-\mathrm{Na}(2) \quad 69.02(5) \\
& \mathrm{N}(8)-\mathrm{Na}(1)-\mathrm{N}(2) \quad 95.67(13) \\
& \mathrm{N}(8)-\mathrm{Na}(1)-\mathrm{N}(4) \quad 74.90(12) \\
& \mathrm{N}(2)-\mathrm{Na}(1)-\mathrm{N}(4) \quad 145.66(14) \\
& \mathrm{N}(8)-\mathrm{Na}(1)-\mathrm{N}(7) \quad 29.27(11) \\
& \mathrm{N}(2)-\mathrm{Na}(1)-\mathrm{N}(7) \quad 67.61(12)
\end{aligned}
$$




$$
\begin{aligned}
& \mathrm{N}(4)-\mathrm{Na}(1)-\mathrm{N}(7) \quad 102.90(12) \\
& \mathrm{N}(8)-\mathrm{Na}(1)-\mathrm{C}(3) \quad 119.58(15) \\
& \mathrm{N}(2)-\mathrm{Na}(1)-\mathrm{C}(3) \quad 30.94(13) \\
& \mathrm{N}(4)-\mathrm{Na}(1)-\mathrm{C}(3) \quad 161.32(16) \\
& \mathrm{N}(7)-\mathrm{Na}(1)-\mathrm{C}(3) \quad 90.39(14) \\
& \mathrm{N}(8)-\mathrm{Na}(1)-\mathrm{C}(39) \quad 89.95(13) \\
& \mathrm{N}(2)-\mathrm{Na}(1)-\mathrm{C}(39) \quad 118.77(15) \\
& \mathrm{N}(4)-\mathrm{Na}(1)-\mathrm{C}(39) \quad 31.02(12) \\
& \mathrm{N}(7)-\mathrm{Na}(1)-\mathrm{C}(39) \quad 110.24(14) \\
& \mathrm{C}(3)-\mathrm{Na}(1)-\mathrm{C}(39) \quad 131.60(17) \\
& \mathrm{N}(8)-\mathrm{Na}(1)-\mathrm{C}(54) \quad 121.66(16) \\
& \mathrm{N}(2)-\mathrm{Na}(1)-\mathrm{C}(54) \quad 130.55(14) \\
& \mathrm{N}(4)-\mathrm{Na}(1)-\mathrm{C}(54) \quad 79.88(13) \\
& \mathrm{N}(7)-\mathrm{Na}(1)-\mathrm{C}(54) \quad 136.37(16) \\
& \mathrm{C}(3)-\mathrm{Na}(1)-\mathrm{C}(54) \quad 99.63(15) \\
& \mathrm{C}(39)-\mathrm{Na}(1)-\mathrm{C}(54) \quad 94.37(15) \\
& \mathrm{N}(8)-\mathrm{Na}(1)-\mathrm{C}(4) \quad 144.15(14) \\
& \mathrm{N}(2)-\mathrm{Na}(1)-\mathrm{C}(4) \quad 48.75(13) \\
& \mathrm{N}(4)-\mathrm{Na}(1)-\mathrm{C}(4) \quad 135.54(14) \\
& \mathrm{N}(7)-\mathrm{Na}(1)-\mathrm{C}(4) \quad 115.15(13) \\
& \mathrm{C}(3)-\mathrm{Na}(1)-\mathrm{C}(4) \quad 26.70(14) \\
& \mathrm{C}(39)-\mathrm{Na}(1)-\mathrm{C}(4) \quad 109.81(15) \\
& \mathrm{C}(54)-\mathrm{Na}(1)-\mathrm{C}(4) \quad 87.38(15) \\
& \mathrm{N}(8)-\mathrm{Na}(1)-\mathrm{Cr}(2) \quad 33.89(8) \\
& \mathrm{N}(2)-\mathrm{Na}(1)-\mathrm{Cr}(2) \quad 121.76(10) \\
& \mathrm{N}(4)-\mathrm{Na}(1)-\mathrm{Cr}(2) \quad 41.08(8) \\
& \mathrm{N}(7)-\mathrm{Na}(1)-\mathrm{Cr}(2) \quad 62.04(9) \\
& \mathrm{C}(3)-\mathrm{Na}(1)-\mathrm{Cr}(2) \quad 151.16(12) \\
& \mathrm{C}(39)-\mathrm{Na}(1)-\mathrm{Cr}(2) \quad 59.40(10)
\end{aligned}
$$




$$
\begin{aligned}
& \mathrm{C}(54)-\mathrm{Na}(1)-\mathrm{Cr}(2) \quad 106.25(11) \\
& \mathrm{C}(4)-\mathrm{Na}(1)-\mathrm{Cr}(2) \quad 162.62(13) \\
& \mathrm{N}(8)-\mathrm{Na}(1)-\mathrm{Cr}(1) \quad 62.03(9) \\
& \mathrm{N}(2)-\mathrm{Na}(1)-\mathrm{Cr}(1) \quad 36.73(8) \\
& \mathrm{N}(4)-\mathrm{Na}(1)-\mathrm{Cr}(1) \quad 133.40(10) \\
& \mathrm{N}(7)-\mathrm{Na}(1)-\mathrm{Cr}(1) \quad 32.76(8) \\
& \mathrm{C}(3)-\mathrm{Na}(1)-\mathrm{Cr}(1) \quad 57.80(11) \\
& \mathrm{C}(39)-\mathrm{Na}(1)-\mathrm{Cr}(1) \quad 127.98(12) \\
& \mathrm{C}(54)-\mathrm{Na}(1)-\mathrm{Cr}(1) \quad 137.41(13) \\
& \mathrm{C}(4)-\mathrm{Na}(1)-\mathrm{Cr}(1) \quad 82.49(10) \\
& \mathrm{Cr}(2)-\mathrm{Na}(1)-\mathrm{Cr}(1) \quad 93.94(5) \\
& \mathrm{N}(8)-\mathrm{Na}(1)-\mathrm{Na}(2) \quad 47.88(9) \\
& \mathrm{N}(2)-\mathrm{Na}(1)-\mathrm{Na}(2) \quad 64.16 \text { (9) } \\
& \mathrm{N}(4)-\mathrm{Na}(1)-\mathrm{Na}(2) \quad 86.57(9) \\
& \mathrm{N}(7)-\mathrm{Na}(1)-\mathrm{Na}(2) \quad 41.63(9) \\
& \mathrm{C}(3)-\mathrm{Na}(1)-\mathrm{Na}(2) \quad 95.10(12) \\
& \mathrm{C}(39)-\mathrm{Na}(1)-\mathrm{Na}(2) \quad 76.56(11) \\
& \mathrm{C}(54)-\mathrm{Na}(1)-\mathrm{Na}(2) \quad 165.22(12) \\
& \mathrm{C}(4)-\mathrm{Na}(1)-\mathrm{Na}(2) \quad 106.57(11) \\
& \mathrm{Cr}(2)-\mathrm{Na}(1)-\mathrm{Na}(2) \quad 59.13(5) \\
& \mathrm{Cr}(1)-\mathrm{Na}(1)-\mathrm{Na}(2) \quad 51.84(4) \\
& \mathrm{N}(7)-\mathrm{Na}(2)-\mathrm{N}(3) \quad 76.84(13) \\
& \mathrm{N}(7)-\mathrm{Na}(2)-\mathrm{N}(5) \quad 90.28(13) \\
& \mathrm{N}(3)-\mathrm{Na}(2)-\mathrm{N}(5) \quad 150.10(14) \\
& \mathrm{N}(7)-\mathrm{Na}(2)-\mathrm{C}(44) \quad 112.95(15) \\
& \mathrm{N}(3)-\mathrm{Na}(2)-\mathrm{C}(44) \quad 168.88(16) \\
& \mathrm{N}(5)-\mathrm{Na}(2)-\mathrm{C}(44) \quad 30.95(12) \\
& \mathrm{N}(7)-\mathrm{Na}(2)-\mathrm{C}(8) \quad 92.68(14)
\end{aligned}
$$




$$
\begin{aligned}
& \mathrm{N}(3)-\mathrm{Na}(2)-\mathrm{C}(8) \quad 31.50(13) \\
& \mathrm{N}(5)-\mathrm{Na}(2)-\mathrm{C}(8) \quad 125.27(15) \\
& \mathrm{C}(44)-\mathrm{Na}(2)-\mathrm{C}(8) \quad 138.88(17) \\
& \mathrm{N}(7)-\mathrm{Na}(2)-\mathrm{N}(8) \quad 27.86(11) \\
& \mathrm{N}(3)-\mathrm{Na}(2)-\mathrm{N}(8) \quad 104.22(12) \\
& \mathrm{N}(5)-\mathrm{Na}(2)-\mathrm{N}(8) \quad 63.65(11) \\
& \mathrm{C}(44)-\mathrm{Na}(2)-\mathrm{N}(8) \quad 85.20(13) \\
& \mathrm{C}(8)-\mathrm{Na}(2)-\mathrm{N}(8) \quad 114.08(14) \\
& \mathrm{N}(7)-\mathrm{Na}(2)-\mathrm{C}(43) \quad 140.86(14) \\
& \mathrm{N}(3)-\mathrm{Na}(2)-\mathrm{C}(43) \quad 140.25(15) \\
& \mathrm{N}(5)-\mathrm{Na}(2)-\mathrm{C}(43) \quad 51.54(12) \\
& \mathrm{C}(44)-\mathrm{Na}(2)-\mathrm{C}(43) \quad 28.97(13) \\
& \mathrm{C}(8)-\mathrm{Na}(2)-\mathrm{C}(43) \quad 115.16(15) \\
& \mathrm{N}(8)-\mathrm{Na}(2)-\mathrm{C}(43) \quad 113.06(13) \\
& \mathrm{N}(7)-\mathrm{Na}(2)-\mathrm{C}(32) \quad 122.65(17) \\
& \mathrm{N}(3)-\mathrm{Na}(2)-\mathrm{C}(32) \quad 78.63(14) \\
& \mathrm{N}(5)-\mathrm{Na}(2)-\mathrm{C}(32) \quad 130.11(15) \\
& \mathrm{C}(44)-\mathrm{Na}(2)-\mathrm{C}(32) \quad 99.20(16) \\
& \mathrm{C}(8)-\mathrm{Na}(2)-\mathrm{C}(32) \quad 92.31(17) \\
& \mathrm{N}(8)-\mathrm{Na}(2)-\mathrm{C}(32) \quad 134.07(17) \\
& \mathrm{C}(43)-\mathrm{Na}(2)-\mathrm{C}(32) \quad 84.97(16) \\
& \mathrm{N}(7)-\mathrm{Na}(2)-\mathrm{Cr}(1) \quad 34.97(9) \\
& \mathrm{N}(3)-\mathrm{Na}(2)-\mathrm{Cr}(1) \quad 42.09 \text { (9) } \\
& \mathrm{N}(5)-\mathrm{Na}(2)-\mathrm{Cr}(1) \quad 119.13(10) \\
& \mathrm{C}(44)-\mathrm{Na}(2)-\mathrm{Cr}(1) \quad 146.90(12) \\
& \mathrm{C}(8)-\mathrm{Na}(2)-\mathrm{Cr}(1) \quad 60.52(11) \\
& \mathrm{N}(8)-\mathrm{Na}(2)-\mathrm{Cr}(1) \quad 62.14 \text { (8) } \\
& \mathrm{C}(43)-\mathrm{Na}(2)-\mathrm{Cr}(1) \quad 166.80(13) \\
& \mathrm{C}(32)-\mathrm{Na}(2)-\mathrm{Cr}(1) \quad 107.23(13)
\end{aligned}
$$




$$
\begin{aligned}
& \mathrm{N}(7)-\mathrm{Na}(2)-\mathrm{C}(40) \quad 94.83(13) \\
& \mathrm{N}(3)-\mathrm{Na}(2)-\mathrm{C}(40) \quad 126.34(14) \\
& \mathrm{N}(5)-\mathrm{Na}(2)-\mathrm{C}(40) \quad 26.89(11) \\
& \mathrm{C}(44)-\mathrm{Na}(2)-\mathrm{C}(40) \quad 49.90(13) \\
& \mathrm{C}(8)-\mathrm{Na}(2)-\mathrm{C}(40) \quad 98.53(15) \\
& \mathrm{N}(8)-\mathrm{Na}(2)-\mathrm{C}(40) \quad 74.56(12) \\
& \mathrm{C}(43)-\mathrm{Na}(2)-\mathrm{C}(40) \quad 56.00(13) \\
& \mathrm{C}(32)-\mathrm{Na}(2)-\mathrm{C}(40) \quad 140.47(16) \\
& \mathrm{Cr}(1)-\mathrm{Na}(2)-\mathrm{C}(40) \quad 111.22(10) \\
& \mathrm{N}(7)-\mathrm{Na}(2)-\mathrm{Cr}(2) \quad 58.39(9) \\
& \mathrm{N}(3)-\mathrm{Na}(2)-\mathrm{Cr}(2) \quad 133.14(10) \\
& \mathrm{N}(5)-\mathrm{Na}(2)-\mathrm{Cr}(2) \quad 34.11(8) \\
& \mathrm{C}(44)-\mathrm{Na}(2)-\mathrm{Cr}(2) \quad 55.22(10) \\
& \mathrm{C}(8)-\mathrm{Na}(2)-\mathrm{Cr}(2) \quad 131.15(12) \\
& \mathrm{N}(8)-\mathrm{Na}(2)-\mathrm{Cr}(2) \quad 30.63(7) \\
& \mathrm{C}(43)-\mathrm{Na}(2)-\mathrm{Cr}(2) \quad 82.47(10) \\
& \mathrm{C}(32)-\mathrm{Na}(2)-\mathrm{Cr}(2) \quad 135.92(14) \\
& \mathrm{Cr}(1)-\mathrm{Na}(2)-\mathrm{Cr}(2) \quad 91.70(5) \\
& \mathrm{C}(40)-\mathrm{Na}(2)-\mathrm{Cr}(2) \quad 52.24(9) \\
& \mathrm{N}(7)-\mathrm{Na}(2)-\mathrm{Na}(1) \quad 44.31(9) \\
& \mathrm{N}(3)-\mathrm{Na}(2)-\mathrm{Na}(1) \quad 87.18(10) \\
& \mathrm{N}(5)-\mathrm{Na}(2)-\mathrm{Na}(1) \quad 65.45(9) \\
& \mathrm{C}(44)-\mathrm{Na}(2)-\mathrm{Na}(1) \quad 96.35(11) \\
& \mathrm{C}(8)-\mathrm{Na}(2)-\mathrm{Na}(1) \quad 79.64(12) \\
& \mathrm{N}(8)-\mathrm{Na}(2)-\mathrm{Na}(1) \quad 41.71(8) \\
& \mathrm{C}(43)-\mathrm{Na}(2)-\mathrm{Na}(1) \quad 111.56(11) \\
& \mathrm{C}(32)-\mathrm{Na}(2)-\mathrm{Na}(1) \quad 163.41(13) \\
& \mathrm{Cr}(1)-\mathrm{Na}(2)-\mathrm{Na}(1) \quad 56.18(4)
\end{aligned}
$$




\begin{tabular}{|c|c|}
\hline $\mathrm{C}(40)-\mathrm{Na}(2)-\mathrm{Na}(1)$ & $55.79(9)$ \\
\hline $\mathrm{Cr}(2)-\mathrm{Na}(2)-\mathrm{Na}(1)$ & $51.85(4)$ \\
\hline$C(2)-N(1)-C(10)$ & $115.5(4)$ \\
\hline$C(2)-N(1)-C r(1)$ & $113.6(3)$ \\
\hline$C(10)-N(1)-C r(1)$ & $130.8(3)$ \\
\hline$C(3)-N(2)-C(7)$ & $121.9(4)$ \\
\hline$C(3)-N(2)-C r(1)$ & $119.6(3)$ \\
\hline$C(7)-N(2)-C r(1)$ & $118.3(3)$ \\
\hline$C(3)-N(2)-N a(1)$ & $76.6(2)$ \\
\hline$C(7)-N(2)-N a(1)$ & $107.0(3)$ \\
\hline $\mathrm{Cr}(1)-\mathrm{N}(2)-\mathrm{Na}(1)$ & $91.33(14)$ \\
\hline$C(8)-N(3)-C(22)$ & $114.5(3)$ \\
\hline$C(8)-N(3)-C r(1)$ & $111.8(3)$ \\
\hline$C(22)-N(3)-C r(1)$ & $127.9(3)$ \\
\hline$C(8)-N(3)-N a(2)$ & $79.7(2)$ \\
\hline $\mathrm{C}(22)-\mathrm{N}(3)-\mathrm{Na}(2)$ & $127.3(3)$ \\
\hline $\mathrm{Cr}(1)-\mathrm{N}(3)-\mathrm{Na}(2)$ & $83.08(13)$ \\
\hline$C(39)-N(4)-C(47)$ & $114.2(3)$ \\
\hline$C(39)-N(4)-C r(2)$ & $111.9(3)$ \\
\hline$C(47)-N(4)-C r(2)$ & $129.5(3)$ \\
\hline $\mathrm{C}(39)-\mathrm{N}(4)-\mathrm{Na}(1)$ & $78.3(2)$ \\
\hline $\mathrm{C}(47)-\mathrm{N}(4)-\mathrm{Na}(1)$ & $124.5(3)$ \\
\hline $\mathrm{Cr}(2)-\mathrm{N}(4)-\mathrm{Na}(1)$ & $84.06(12)$ \\
\hline$C(44)-N(5)-C(40)$ & $122 . \odot(4)$ \\
\hline$C(44)-N(5)-C r(2)$ & $119.9(3)$ \\
\hline$C(40)-N(5)-C r(2)$ & $118.1(3)$ \\
\hline $\mathrm{C}(44)-\mathrm{N}(5)-\mathrm{Na}(2)$ & $75.3(2)$ \\
\hline $\mathrm{C}(40)-\mathrm{N}(5)-\mathrm{Na}(2)$ & $97.1(2)$ \\
\hline $\mathrm{Cr}(2)-\mathrm{N}(5)-\mathrm{Na}(2)$ & $97.86(14)$ \\
\hline
\end{tabular}




\begin{tabular}{|c|c|}
\hline$C(45)-N(6)-C(59)$ & $115.5(3)$ \\
\hline$C(45)-N(6)-C r(2)$ & $114.2(3)$ \\
\hline$C(59)-N(6)-C r(2)$ & $129.8(3)$ \\
\hline$N(8)-N(7)-C r(1)$ & $163.8(3)$ \\
\hline$N(8)-N(7)-N a(2)$ & $88.3(2)$ \\
\hline $\mathrm{Cr}(1)-\mathrm{N}(7)-\mathrm{Na}(2)$ & $91.41(15)$ \\
\hline$N(8)-N(7)-N a(1)$ & $69.8(2)$ \\
\hline $\mathrm{Cr}(1)-\mathrm{N}(7)-\mathrm{Na}(1)$ & $94.15(14)$ \\
\hline $\mathrm{Na}(2)-\mathrm{N}(7)-\mathrm{Na}(1)$ & $94.06(13)$ \\
\hline$N(7)-N(8)-C r(2)$ & $159.0(3)$ \\
\hline$N(7)-N(8)-N a(1)$ & $81.0(2)$ \\
\hline $\mathrm{Cr}(2)-\mathrm{N}(8)-\mathrm{Na}(1)$ & $94.11(15)$ \\
\hline$N(7)-N(8)-N a(2)$ & $63.8(2)$ \\
\hline $\mathrm{Cr}(2)-\mathrm{N}(8)-\mathrm{Na}(2)$ & $96.0 \odot(14)$ \\
\hline $\mathrm{Na}(1)-\mathrm{N}(8)-\mathrm{Na}(2)$ & $90.41(13)$ \\
\hline$C(37)-0(1)-C(34)$ & $108.6(3)$ \\
\hline$C(37)-0(1)-C r(1)$ & $123.9(3)$ \\
\hline$C(34)-0(1)-C r(1)$ & $125.4(3)$ \\
\hline$C(74)-0(2)-C(71)$ & $108.4(3)$ \\
\hline$C(74)-0(2)-C r(2)$ & $122.5(3)$ \\
\hline$C(71)-0(2)-C r(2)$ & $124.7(3)$ \\
\hline$N(1)-C(2)-C(3)$ & $114.7(4)$ \\
\hline$N(1)-C(2)-C(1)$ & $123.1(4)$ \\
\hline$C(3)-C(2)-C(1)$ & $122.2(4)$ \\
\hline$N(2)-C(3)-C(2)$ & $112.1(4)$ \\
\hline$N(2)-C(3)-C(4)$ & $118.4(4)$ \\
\hline$C(2)-C(3)-C(4)$ & $129.6(4)$ \\
\hline$N(2)-C(3)-N a(1)$ & $72.5(2)$ \\
\hline
\end{tabular}




\begin{tabular}{|c|c|}
\hline$C(2)-C(3)-N a(1)$ & $98.2(3)$ \\
\hline $\mathrm{C}(4)-\mathrm{C}(3)-\mathrm{Na}(1)$ & $97.0(3)$ \\
\hline$c(5)-c(4)-c(3)$ & $121.0(5)$ \\
\hline$C(5)-C(4)-\mathrm{Na}(1)$ & $97.6(3)$ \\
\hline $\mathrm{C}(3)-\mathrm{C}(4)-\mathrm{Na}(1)$ & $56.3(2)$ \\
\hline$C(4)-C(5)-C(6)$ & $122.8(5)$ \\
\hline$C(5)-C(6)-C(7)$ & $115.6(5)$ \\
\hline$C(8)-C(7)-N(2)$ & $113.6(4)$ \\
\hline$C(8)-C(7)-C(6)$ & $127.6(4)$ \\
\hline$N(2)-C(7)-C(6)$ & $118.7(4)$ \\
\hline$C(7)-C(8)-N(3)$ & $114.9(4)$ \\
\hline$C(7)-C(8)-C(9)$ & $123.8(4)$ \\
\hline$N(3)-C(8)-C(9)$ & $121.2(4)$ \\
\hline $\mathrm{C}(7)-\mathrm{C}(8)-\mathrm{Na}(2)$ & $100.2(3)$ \\
\hline$N(3)-C(8)-N a(2)$ & $68.8(2)$ \\
\hline $\mathrm{C}(9)-\mathrm{C}(8)-\mathrm{Na}(2)$ & $97.4(3)$ \\
\hline$C(15)-C(10)-C(11)$ & $120.3(4)$ \\
\hline$C(15)-C(10)-N(1)$ & $120.3(4)$ \\
\hline$C(11)-C(10)-N(1)$ & $119.4(4)$ \\
\hline$C(12)-C(11)-C(10)$ & $117.5(5)$ \\
\hline$C(12)-C(11)-C(16)$ & $119.3(4)$ \\
\hline$C(10)-C(11)-C(16)$ & $123.2(4)$ \\
\hline$C(13)-C(12)-C(11)$ & $122.9(5)$ \\
\hline$C(12)-C(13)-C(14)$ & $119 . \odot(5)$ \\
\hline$C(13)-C(14)-C(15)$ & $121.4(5)$ \\
\hline$C(14)-C(15)-C(10)$ & $118.5(4)$ \\
\hline$C(14)-C(15)-C(19)$ & $119.2(5)$ \\
\hline$C(10)-C(15)-C(19)$ & $122.2(4)$ \\
\hline$C(17)-C(16)-C(18)$ & $108.0(5)$ \\
\hline
\end{tabular}




$$
\begin{aligned}
& C(17)-C(16)-C(11) \quad 113.0(5) \\
& C(18)-C(16)-C(11) \quad 111.9(4) \\
& C(21)-C(19)-C(20) \quad 110.2(4) \\
& C(21)-C(19)-C(15) \quad 114.8(4) \\
& C(20)-C(19)-C(15) \quad 110.7(4) \\
& \mathrm{C}(27)-\mathrm{C}(22)-\mathrm{C}(23) \quad 119.2(4) \\
& \mathrm{C}(27)-\mathrm{C}(22)-\mathrm{N}(3) \quad 121.0(4) \\
& \mathrm{C}(23)-\mathrm{C}(22)-\mathrm{N}(3) \quad 119.8(4) \\
& \mathrm{C}(24)-\mathrm{C}(23)-\mathrm{C}(22) \quad 118.5(4) \\
& C(24)-C(23)-C(28) \quad 118.2(4) \\
& C(22)-C(23)-C(28) \quad 123.3(4) \\
& C(25)-C(24)-C(23) \quad 122.3(5) \\
& C(24)-C(25)-C(26) \quad 118.9(5) \\
& \mathrm{C}(25)-\mathrm{C}(26)-\mathrm{C}(27) \quad 121.9(5) \\
& \mathrm{C}(26)-\mathrm{C}(27)-\mathrm{C}(22) \quad 119.1(4) \\
& C(26)-C(27)-C(31) \quad 118.7(4) \\
& C(22)-C(27)-C(31) \quad 122.1(4) \\
& C(23)-C(28)-C(30) \quad 111.6(4) \\
& C(23)-C(28)-C(29) \quad 113.1(5) \\
& C(30)-C(28)-C(29) \quad 108.8(4) \\
& \mathrm{C}(27)-\mathrm{C}(31)-\mathrm{C}(33) \quad 112.9(4) \\
& C(27)-C(31)-C(32) \quad 110.4(5) \\
& C(33)-C(31)-C(32) \quad 110.1(5) \\
& \mathrm{C}(31)-\mathrm{C}(32)-\mathrm{Na}(2) \quad 83.1(3) \\
& \mathrm{O}(1)-\mathrm{C}(34)-\mathrm{C}(35) \quad 106.3(4) \\
& \mathrm{C}(36)-\mathrm{C}(35)-\mathrm{C}(34) \quad 107.8(5) \\
& \mathrm{C}(35)-\mathrm{C}(36)-\mathrm{C}(37) \quad 105.6(5) \\
& 0(1)-C(37)-C(36) \quad 105.1(4)
\end{aligned}
$$




$$
\begin{aligned}
& \mathrm{C}(40)-\mathrm{C}(39)-\mathrm{N}(4) \quad 115.3(4) \\
& C(40)-C(39)-C(38) \quad 123.2(4) \\
& \mathrm{N}(4)-\mathrm{C}(39)-\mathrm{C}(38) \quad 121.5(4) \\
& \mathrm{C}(40)-\mathrm{C}(39)-\mathrm{Na}(1) \quad 100.7(3) \\
& \mathrm{N}(4)-\mathrm{C}(39)-\mathrm{Na}(1) \quad 70.7(2) \\
& \mathrm{C}(38)-\mathrm{C}(39)-\mathrm{Na}(1) \quad 95.9 \text { (3) } \\
& C(39)-C(40)-N(5) \quad 112.4(4) \\
& C(39)-C(40)-C(41) \quad 129.1(4) \\
& \mathrm{N}(5)-\mathrm{C}(40)-\mathrm{C}(41) \quad 118.4(4) \\
& \mathrm{C}(39)-\mathrm{C}(40)-\mathrm{Na}(2) \quad 126.1(3) \\
& \mathrm{N}(5)-\mathrm{C}(4 \odot)-\mathrm{Na}(2) \quad 56 . \odot(2) \\
& \mathrm{C}(41)-\mathrm{C}(40)-\mathrm{Na}(2) \quad 85.9(3) \\
& C(42)-C(41)-C(40) \quad 116.6(4) \\
& C(43)-C(42)-C(41) \quad 122.2(4) \\
& C(42)-C(43)-C(44) \quad 120.6(4) \\
& \mathrm{C}(42)-\mathrm{C}(43)-\mathrm{Na}(2) \quad 89.3(3) \\
& \mathrm{C}(44)-\mathrm{C}(43)-\mathrm{Na}(2) \quad 63.8(2) \\
& N(5)-C(44)-C(43) \quad 118.7(4) \\
& \mathrm{N}(5)-\mathrm{C}(44)-\mathrm{C}(45) \quad 112.0(4) \\
& C(43)-C(44)-C(45) \quad 129.3(4) \\
& \mathrm{N}(5)-\mathrm{C}(44)-\mathrm{Na}(2) \quad 73.8(2) \\
& \mathrm{C}(43)-\mathrm{C}(44)-\mathrm{Na}(2) \quad 87.2(3) \\
& \mathrm{C}(45)-\mathrm{C}(44)-\mathrm{Na}(2) \quad 106.1(3) \\
& \mathrm{N}(6)-\mathrm{C}(45)-\mathrm{C}(44) \quad 113.6(4) \\
& \mathrm{N}(6)-\mathrm{C}(45)-\mathrm{C}(46) \quad 124.6(4) \\
& C(44)-C(45)-C(46) \quad 121.8(4) \\
& C(48)-C(47)-C(52) \quad 118.9(4) \\
& \mathrm{C}(48)-\mathrm{C}(47)-\mathrm{N}(4) \quad 121.2(4) \\
& \mathrm{C}(52)-\mathrm{C}(47)-\mathrm{N}(4) \quad 119.9(4)
\end{aligned}
$$




$$
\begin{aligned}
& C(49)-C(48)-C(47) \quad 119.6(4) \\
& C(49)-C(48)-C(53) \quad 118.6(4) \\
& C(47)-C(48)-C(53) \quad 121.8(4) \\
& C(50)-C(49)-C(48) \quad 121.7(5) \\
& C(49)-C(50)-C(51) \quad 119 . \odot(4) \\
& C(50)-C(51)-C(52) \quad 121.9(4) \\
& C(51)-C(52)-C(47) \quad 118.7(4) \\
& C(51)-C(52)-C(56) \quad 117.7(4) \\
& C(47)-C(52)-C(56) \quad 123.5(4) \\
& C(48)-C(53)-C(55) \quad 112.4(4) \\
& C(48)-C(53)-C(54) \quad 110.0(4) \\
& C(55)-C(53)-C(54) \quad 111.3(4) \\
& \mathrm{C}(53)-\mathrm{C}(54)-\mathrm{Na}(1) \quad 80.1(3) \\
& \mathrm{C}(52)-\mathrm{C}(56)-\mathrm{C}(58) \quad 111.1(4) \\
& C(52)-C(56)-C(57) \quad 111.9(4) \\
& C(58)-C(56)-C(57) \quad 109.3(4) \\
& C(64)-C(59)-C(60) \quad 120.2(4) \\
& \mathrm{C}(64)-\mathrm{C}(59)-\mathrm{N}(6) \quad 120.4(4) \\
& \mathrm{C}(60)-\mathrm{C}(59)-\mathrm{N}(6) \quad 119.5(4) \\
& C(61)-C(60)-C(59) \quad 118.6(4) \\
& C(61)-C(60)-C(65) \quad 118.4(4) \\
& C(59)-C(6 \odot)-C(65) \quad 123 . \odot(4) \\
& C(62)-C(61)-C(60) \quad 121.9(4) \\
& C(61)-C(62)-C(63) \quad 119.1(5) \\
& C(62)-C(63)-C(64) \quad 121.8(5) \\
& C(59)-C(64)-C(63) \quad 118.5(4) \\
& C(59)-C(64)-C(68) \quad 123.3(4) \\
& C(63)-C(64)-C(68) \quad 118.1(4)
\end{aligned}
$$




$$
\begin{array}{lr}
C(60)-C(65)-C(67) & 111.7(4) \\
C(60)-C(65)-C(66) & 112.3(4) \\
C(67)-C(65)-C(66) & 108.2(4) \\
C(70)-C(68)-C(69) & 107.0(5) \\
C(70)-C(68)-C(64) & 110.4(5) \\
C(69)-C(68)-C(64) & 114.3(4) \\
O(2)-C(71)-C(72) & 105.1(4) \\
C(71)-C(72)-C(73) & 103.1(4) \\
C(74)-C(73)-C(72) & 103.6(5) \\
0(2)-C(74)-C(73) & 108.1(4)
\end{array}
$$

Symmetry transformations used to generate equivalent atoms: 
Table S16. Anisotropic displacement parameters $\left(A^{\wedge} 2 \times 10^{\wedge} 3\right)$ for 6 . The anisotropic displacement factor exponent takes the form:

$-2 \operatorname{pi} 2\left[h^{\wedge} 2 a^{* \wedge} \mathrm{U} 11+\ldots+2 h k a^{*} b^{*} \mathrm{U} 12\right]$

U11

U22

U33

$\mathrm{U} 23$

U13

U12

\begin{tabular}{|c|c|c|c|c|c|c|}
\hline $\operatorname{cr}(1)$ & $27(1)$ & $34(1)$ & $28(1)$ & 1(1) & $8(1)$ & $\odot(1)$ \\
\hline $\operatorname{cr}(2)$ & $25(1)$ & $31(1)$ & $28(1)$ & $\odot(1)$ & $7(1)$ & $-1(1)$ \\
\hline $\mathrm{Na}(1)$ & $34(1)$ & $73(2)$ & $35(1)$ & $16(1)$ & $13(1)$ & $8(1)$ \\
\hline $\mathrm{Na}(2)$ & $43(1)$ & $73(2)$ & $36(1)$ & $-17(1)$ & $19(1)$ & $-19(1)$ \\
\hline$N(1)$ & $26(2)$ & $37(2)$ & $29(2)$ & $1(2)$ & $6(2)$ & $-2(2)$ \\
\hline$N(2)$ & $26(2)$ & $33(2)$ & $28(2)$ & $2(2)$ & $7(2)$ & $-2(2)$ \\
\hline $\mathrm{N}(3)$ & $25(2)$ & $33(2)$ & $32(2)$ & $-3(2)$ & $8(2)$ & $-1(2)$ \\
\hline $\mathrm{N}(4)$ & $19(2)$ & $30(2)$ & $33(2)$ & $5(2)$ & $6(2)$ & $-1(2)$ \\
\hline$N(5)$ & $24(2)$ & $29(2)$ & $27(2)$ & $4(2)$ & $6(2)$ & $\odot(2)$ \\
\hline$N(6)$ & $29(2)$ & $25(2)$ & $27(2)$ & 1(2) & $5(2)$ & $-2(2)$ \\
\hline $\mathrm{N}(7)$ & $23(2)$ & $29(2)$ & $28(2)$ & $-4(2)$ & $4(2)$ & $5(2)$ \\
\hline$N(8)$ & $24(2)$ & $29(2)$ & $24(2)$ & $\odot(2)$ & $6(2)$ & $-1(2)$ \\
\hline $0(1)$ & $35(2)$ & $35(2)$ & $35(2)$ & $8(2)$ & 18(2) & $9(2)$ \\
\hline $0(2)$ & $32(2)$ & $32(2)$ & $41(2)$ & $-4(2)$ & 16(2) & $-8(1)$ \\
\hline$c(1)$ & $35(3)$ & $62(4)$ & $37(3)$ & $\odot(3)$ & $4(2)$ & $4(3)$ \\
\hline$C(2)$ & $24(3)$ & $46(3)$ & $27(3)$ & $7(2)$ & $5(2)$ & $2(2)$ \\
\hline$C(3)$ & $31(3)$ & $41(3)$ & $29(3)$ & $4(2)$ & $12(2)$ & $2(2)$ \\
\hline$c(4)$ & $39(3)$ & $51(4)$ & $38(3)$ & 13(3) & $2(2)$ & $11(3)$ \\
\hline$c(5)$ & $52(4)$ & $37(3)$ & $61(4)$ & $12(3)$ & $24(3)$ & $15(3)$ \\
\hline
\end{tabular}




\begin{tabular}{|c|c|c|c|c|c|c|}
\hline$C(6)$ & $45(3)$ & $33(3)$ & $54(3)$ & $-2(2)$ & $13(3)$ & $4(2)$ \\
\hline$c(7)$ & $32(3)$ & $32(3)$ & $35(3)$ & $3(2)$ & $17(2)$ & $-1(2)$ \\
\hline$C(8)$ & $34(3)$ & $34(3)$ & $39(3)$ & $-6(2)$ & $18(2)$ & $-8(2)$ \\
\hline$c(9)$ & $40(3)$ & $47(3)$ & $59(4)$ & $-9(3)$ & $19(3)$ & $-5(3)$ \\
\hline$c(10)$ & $22(2)$ & $42(3)$ & $29(3)$ & $-6(2)$ & $1(2)$ & $\Theta(2)$ \\
\hline$c(11)$ & $29(3)$ & $53(3)$ & $32(3)$ & $-4(3)$ & $7(2)$ & $3(2)$ \\
\hline$C(12)$ & $48(3)$ & $66(4)$ & $33(3)$ & $-9(3)$ & $16(3)$ & $3(3)$ \\
\hline$c(13)$ & $67(4)$ & $56(4)$ & $53(4)$ & $-24(3)$ & $27(3)$ & $-8(3)$ \\
\hline$C(14)$ & $53(3)$ & $46(4)$ & $54(4)$ & $-10(3)$ & $19(3)$ & $-5(3)$ \\
\hline$C(15)$ & $36(3)$ & $38(3)$ & $35(3)$ & $-9(2)$ & $8(2)$ & $-2(2)$ \\
\hline$c(16)$ & $46(3)$ & $53(4)$ & $46(3)$ & $5(3)$ & $22(3)$ & $4(3)$ \\
\hline$C(17)$ & $63(5)$ & $136(7)$ & $132(7)$ & $88(6)$ & $39(5)$ & $21(5)$ \\
\hline$C(18)$ & $48(3)$ & $68(4)$ & $49(3)$ & $-5(3)$ & $19(3)$ & $-9(3)$ \\
\hline$c(19)$ & $46(3)$ & $36(3)$ & $38(3)$ & $-6(2)$ & $15(2)$ & $-3(2)$ \\
\hline$C(20)$ & $57(4)$ & $66(4)$ & $57(4)$ & $-2(3)$ & $29(3)$ & $-4(3)$ \\
\hline$C(21)$ & $66(4)$ & $5 \odot(4)$ & $48(3)$ & $-3(3)$ & $16(3)$ & $-8(3)$ \\
\hline$C(22)$ & $24(3)$ & $28(3)$ & $34(3)$ & $-6(2)$ & $10(2)$ & $-5(2)$ \\
\hline$c(23)$ & $35(3)$ & $31(3)$ & $37(3)$ & $-5(2)$ & $13(2)$ & $-2(2)$ \\
\hline$c(24)$ & $4 \odot(3)$ & $42(3)$ & $50(3)$ & $1(3)$ & $25(3)$ & $-6(2)$ \\
\hline$c(25)$ & $24(3)$ & $57(4)$ & $64(4)$ & $1(3)$ & $14(3)$ & $-5(3)$ \\
\hline$C(26)$ & $29(3)$ & $59(4)$ & $49(3)$ & $6(3)$ & $8(3)$ & $\theta(3)$ \\
\hline$c(27)$ & $29(3)$ & $42(3)$ & $33(3)$ & $-1(2)$ & $6(2)$ & $-3(2)$ \\
\hline$c(28)$ & $36(3)$ & $56(4)$ & $40(3)$ & $12(3)$ & $17(2)$ & $1(3)$ \\
\hline$C(29)$ & $86(5)$ & $55(4)$ & $68(4)$ & $23(3)$ & $27(4)$ & $21(3)$ \\
\hline$c(30)$ & $57(4)$ & $77(4)$ & $44(3)$ & $5(3)$ & $12(3)$ & $-3(3)$ \\
\hline$c(31)$ & $32(3)$ & $67(4)$ & $33(3)$ & $6(3)$ & $1(2)$ & $-10(3)$ \\
\hline$C(32)$ & $74(4)$ & $104(6)$ & $52(4)$ & $-9(4)$ & $23(3)$ & $-30(4)$ \\
\hline$c(33)$ & $45(4)$ & $92(5)$ & $7 \odot(4)$ & $42(4)$ & $-7(3)$ & $-6(3)$ \\
\hline
\end{tabular}




\begin{tabular}{|c|c|c|c|c|c|c|}
\hline$c(34)$ & $4 \odot(3)$ & $40(3)$ & $43(3)$ & $6(2)$ & $23(2)$ & $9(2)$ \\
\hline$c(35)$ & $146(7)$ & $56(4)$ & $125(6)$ & $25(4)$ & $106(6)$ & $33(5)$ \\
\hline$C(36)$ & $103(6)$ & $51(4)$ & $137(7)$ & $24(4)$ & $80(5)$ & $32(4)$ \\
\hline$c(37)$ & $43(3)$ & $45(3)$ & $55(3)$ & $19(3)$ & $18(3)$ & $8(3)$ \\
\hline$C(38)$ & $40(3)$ & $42(3)$ & $52(3)$ & $9(3)$ & $11(3)$ & $3(3)$ \\
\hline$C(39)$ & $31(3)$ & $34(3)$ & $26(3)$ & $7(2)$ & $10(2)$ & $4(2)$ \\
\hline$c(40)$ & $30(3)$ & $29(3)$ & $29(3)$ & $3(2)$ & $9(2)$ & $\Theta(2)$ \\
\hline$c(41)$ & $41(3)$ & $27(3)$ & $41(3)$ & $4(2)$ & $8(2)$ & $-1(2)$ \\
\hline$c(42)$ & $47(3)$ & $29(3)$ & $42(3)$ & $-5(2)$ & $17(3)$ & $-11(2)$ \\
\hline$c(43)$ & $32(3)$ & $35(3)$ & $30(3)$ & $-5(2)$ & $3(2)$ & $-9(2)$ \\
\hline$c(44)$ & $29(3)$ & $34(3)$ & $23(3)$ & $1(2)$ & $8(2)$ & $-1(2)$ \\
\hline$c(45)$ & $27(3)$ & $36(3)$ & $27(3)$ & $-2(2)$ & $10(2)$ & $-1(2)$ \\
\hline$C(46)$ & $43(3)$ & $47(3)$ & $37(3)$ & $7(2)$ & $-5(2)$ & $-12(3)$ \\
\hline$C(47)$ & $24(3)$ & $32(3)$ & $29(3)$ & $7(2)$ & $3(2)$ & $2(2)$ \\
\hline$C(48)$ & $26(3)$ & $43(3)$ & $31(3)$ & $3(2)$ & $3(2)$ & $1(2)$ \\
\hline$C(49)$ & $28(3)$ & $67(4)$ & $39(3)$ & $-12(3)$ & $3(2)$ & $-8(3)$ \\
\hline$C(50)$ & $22(3)$ & $65(4)$ & $48(3)$ & $-1(3)$ & $6(2)$ & $-5(3)$ \\
\hline$c(51)$ & $32(3)$ & $44(3)$ & $41(3)$ & $-3(2)$ & $14(2)$ & $3(2)$ \\
\hline$C(52)$ & $28(3)$ & $33(3)$ & $30(3)$ & $5(2)$ & $10(2)$ & $1(2)$ \\
\hline$c(53)$ & $30(3)$ & $57(4)$ & $33(3)$ & $-8(3)$ & $3(2)$ & $-2(2)$ \\
\hline$C(54)$ & $54(4)$ & $87(5)$ & $4 \odot(3)$ & $3(3)$ & $13(3)$ & $17(3)$ \\
\hline$c(55)$ & $43(3)$ & $77(5)$ & $6 \odot(4)$ & $-28(3)$ & $6(3)$ & $-7(3)$ \\
\hline$C(56)$ & $26(3)$ & $43(3)$ & $37(3)$ & $-4(2)$ & $7(2)$ & $2(2)$ \\
\hline$C(57)$ & $52(4)$ & $52(4)$ & $63(4)$ & $-12(3)$ & $6(3)$ & $-2(3)$ \\
\hline$C(58)$ & $49(3)$ & $74(4)$ & $34(3)$ & $-1(3)$ & $8(3)$ & $2(3)$ \\
\hline$C(59)$ & $23(2)$ & $27(3)$ & $29(3)$ & $4(2)$ & $-2(2)$ & $-3(2)$ \\
\hline$c(60)$ & $23(2)$ & $34(3)$ & $27(3)$ & $6(2)$ & $4(2)$ & $1(2)$ \\
\hline$C(61)$ & $46(3)$ & $40(3)$ & $40(3)$ & $11(2)$ & $17(2)$ & $5(3)$ \\
\hline$C(62)$ & $57(3)$ & $35(3)$ & $47(3)$ & $19(3)$ & $17(3)$ & $5(3)$ \\
\hline
\end{tabular}




\begin{tabular}{lrrrrrr}
$C(63)$ & $46(3)$ & $37(3)$ & $46(3)$ & $1(3)$ & $17(3)$ & $9(2)$ \\
$C(64)$ & $31(3)$ & $29(3)$ & $35(3)$ & $2(2)$ & $8(2)$ & $-1(2)$ \\
$C(65)$ & $37(3)$ & $40(3)$ & $32(3)$ & $9(2)$ & $9(2)$ & $5(2)$ \\
$C(66)$ & $37(3)$ & $65(4)$ & $44(3)$ & $9(3)$ & $21(3)$ & $7(3)$ \\
$C(67)$ & $58(4)$ & $45(3)$ & $51(3)$ & $-5(3)$ & $16(3)$ & $4(3)$ \\
$C(68)$ & $58(4)$ & $41(3)$ & $62(4)$ & $14(3)$ & $38(3)$ & $14(3)$ \\
$C(69)$ & $64(4)$ & $79(5)$ & $57(4)$ & $-9(3)$ & $31(3)$ & $-2(3)$ \\
$C(70)$ & $88(6)$ & $185(10)$ & $143(8)$ & $-90(7)$ & $84(6)$ & $-74(6)$ \\
$C(71)$ & $42(3)$ & $42(3)$ & $50(3)$ & $0(2)$ & $23(3)$ & $-4(2)$ \\
$C(72)$ & $60(4)$ & $47(4)$ & $59(4)$ & $4(3)$ & $24(3)$ & $-9(3)$ \\
$C(73)$ & $80(4)$ & $40(4)$ & $79(4)$ & $2(3)$ & $35(4)$ & $-3(3)$ \\
$C(74)$ & $61(4)$ & $37(3)$ & $75(4)$ & $-18(3)$ & $35(3)$ & $-7(3)$ \\
\hline
\end{tabular}


Table S17. Hydrogen coordinates ( $x$ 10^4) and isotropic displacement parameters $\left(A^{\wedge} 2 \times 10^{\wedge} 3\right)$ for 6 .

$\begin{array}{llll}\text { x } & y & \mathrm{z} & \mathrm{U}(\mathrm{eq})\end{array}$

\begin{tabular}{|c|c|c|c|c|}
\hline$H(7 A)$ & 5350 & 7509 & 2488 & $4 \odot$ \\
\hline$H(1 A)$ & 3729 & 7677 & 283 & 68 \\
\hline$H(1 B)$ & 3614 & 8500 & 88 & 68 \\
\hline $\mathrm{H}(1 \mathrm{C})$ & 3190 & 8165 & 594 & 68 \\
\hline$H(4 A)$ & 3431 & 9604 & 807 & 53 \\
\hline$H(5 A)$ & 3692 & 10631 & 1391 & 58 \\
\hline$H(6 A)$ & 4805 & 10754 & 2239 & 53 \\
\hline$H(9 A)$ & 5977 & 10684 & 2829 & 71 \\
\hline$H(9 B)$ & 6772 & 10401 & 2744 & 71 \\
\hline$H(9 C)$ & 6445 & 10130 & 3334 & 71 \\
\hline$H(12 A)$ & 5280 & 6758 & -555 & 58 \\
\hline$H(13 A)$ & 4878 & 5667 & -273 & 68 \\
\hline $\mathrm{H}(14 \mathrm{~A})$ & 4550 & 5535 & 717 & $6 \odot$ \\
\hline$H(16 A)$ & 5623 & 8339 & 413 & 56 \\
\hline$H(17 A)$ & 4486 & 8498 & -366 & 161 \\
\hline$H(17 B)$ & 4846 & 8110 & -889 & 161 \\
\hline$H(17 C)$ & 5172 & 8860 & -594 & 161 \\
\hline$H(18 A)$ & 6596 & 7648 & 142 & 80 \\
\hline$H(18 B)$ & 6435 & 8349 & -293 & 80 \\
\hline$H(18 C)$ & 6122 & 7593 & -583 & 80 \\
\hline
\end{tabular}




\begin{tabular}{|c|c|c|c|c|}
\hline$H(19 A)$ & 4786 & 6791 & 1967 & 47 \\
\hline$H(2 \odot A)$ & 3590 & 7174 & 1400 & 86 \\
\hline$H(20 B)$ & 3533 & 6678 & 1994 & 86 \\
\hline$H(20 C)$ & 3331 & 6360 & 1283 & 86 \\
\hline$H(21 A)$ & 5121 & 5571 & 1970 & 81 \\
\hline$H(21 B)$ & 4273 & 5373 & 1635 & 81 \\
\hline$H(21 C)$ & 4470 & 5697 & 2344 & 81 \\
\hline$H(24 A)$ & 8229 & 9719 & 1635 & 50 \\
\hline$H(25 A)$ & 9143 & 9263 & 2474 & 57 \\
\hline$H(26 A)$ & 8785 & 8609 & 3279 & 55 \\
\hline$H(28 A)$ & 6244 & 9526 & 1394 & 51 \\
\hline$H(29 A)$ & 6620 & 10702 & 1746 & 103 \\
\hline$H(29 B)$ & 6336 & 10675 & 983 & 103 \\
\hline$H(29 C)$ & 7214 & 10703 & 1303 & 103 \\
\hline$H(30 A)$ & 6927 & 8897 & 747 & 89 \\
\hline$H(30 B)$ & 7401 & 9602 & 693 & 89 \\
\hline$H(30 \mathrm{C})$ & 6521 & 9582 & 378 & 89 \\
\hline$H(31 A)$ & 6949 & 8102 & 3363 & 54 \\
\hline$H(32 A)$ & 7075 & 9209 & 3926 & 112 \\
\hline$H(32 B)$ & 7927 & 9019 & 4256 & 112 \\
\hline$H(32 C)$ & 7261 & 8556 & 4418 & 112 \\
\hline$H(33 A)$ & 8027 & 7375 & 3398 & 108 \\
\hline$H(33 B)$ & 7839 & 7425 & $409 \odot$ & 108 \\
\hline$H(33 C)$ & 8510 & 7886 & 3935 & 108 \\
\hline$H(34 A)$ & 7322 & 7728 & 1762 & 47 \\
\hline$H(34 B)$ & 6607 & 7530 & 1199 & 47 \\
\hline$H(35 A)$ & 7087 & 6469 & 1185 & 116 \\
\hline$H(35 B)$ & 7714 & 6629 & 1827 & 116 \\
\hline
\end{tabular}




\begin{tabular}{|c|c|c|c|}
\hline$H(36 A)$ & 7093 & 5858 & 2253 \\
\hline$H(36 B)$ & 6338 & 5970 & 1706 \\
\hline$H(37 A)$ & 5980 & 6578 & 2487 \\
\hline$H(37 B)$ & 6829 & 6765 & 2848 \\
\hline$H(38 A)$ & 3313 & 10340 & 2930 \\
\hline$H(38 B)$ & 2577 & 9929 & 3015 \\
\hline$H(38 C)$ & 2952 & 9730 & 2432 \\
\hline$H(41 A)$ & 4479 & 10571 & 3512 \\
\hline$H(42 A)$ & 5623 & 10604 & 4320 \\
\hline$H(43 A)$ & 6039 & 9622 & 4957 \\
\hline$H(46 A)$ & 6140 & 7721 & 5576 \\
\hline$H(46 B)$ & 6366 & 8560 & 5501 \\
\hline$H(49 A)$ & 933 & 7849 & 2435 \\
\hline$H(5 \odot A)$ & 411 & 8444 & 3184 \\
\hline$H(51 A)$ & 1188 & 9061 & 4022 \\
\hline$H(53 A)$ & 2870 & 7688 & 2434 \\
\hline$H(54 A)$ & 2446 & 8705 & 1790 \\
\hline$H(54 B)$ & 1675 & 8307 & 1486 \\
\hline$H(54 C)$ & 2445 & 8015 & 1351 \\
\hline$H(55 A)$ & 2046 & 6729 & 2488 \\
\hline$H(55 B)$ & 2191 & 6793 & 1781 \\
\hline$H(55 C)$ & 1423 & 7080 & 1925 \\
\hline$H(56 A)$ & 3189 & 9217 & 4338 \\
\hline$H(57 A)$ & 2494 & 10276 & 3976 \\
\hline $\mathrm{H}(57 \mathrm{~B})$ & 2766 & 10278 & 4740 \\
\hline$H(57 C)$ & 1915 & 10086 & 4409 \\
\hline $\mathrm{H}(58 \mathrm{~A})$ & 2702 & 8382 & 4980 \\
\hline$H(58 B)$ & 2048 & 8930 & 5027 \\
\hline$H(58 C)$ & 2900 & 9122 & 5352 \\
\hline
\end{tabular}




\begin{tabular}{|c|c|c|c|}
\hline$H(61 A)$ & 4584 & 6274 & 6221 \\
\hline$H(62 A)$ & 5327 & 5366 & 5959 \\
\hline$H(63 A)$ & 5821 & 5480 & 5053 \\
\hline$H(65 A)$ & 4038 & 7894 & 5359 \\
\hline$H(66 A)$ & 3175 & 6978 & 5497 \\
\hline$H(66 B)$ & 3158 & 7629 & 5976 \\
\hline$H(66 C)$ & 3594 & 6911 & 6236 \\
\hline$H(67 A)$ & 5085 & 8145 & 6223 \\
\hline$H(67 B)$ & 4756 & 7613 & 6675 \\
\hline$H(67 C)$ & 4315 & 8331 & 6420 \\
\hline$H(68 A)$ & 5642 & 6976 & 3985 \\
\hline$H(69 A)$ & 5212 & 5824 & 3608 \\
\hline$H(69 B)$ & 5945 & 5472 & 4051 \\
\hline$H(69 C)$ & 6017 & 5976 & 3466 \\
\hline$H(7 \odot A)$ & 6762 & 7065 & 4798 \\
\hline$H(7 \odot B)$ & 6928 & 6719 & 4162 \\
\hline $\mathrm{H}(7 \odot \mathrm{C})$ & 6884 & 6223 & 4761 \\
\hline$H(71 A)$ & 2411 & 7049 & 3882 \\
\hline$H(71 B)$ & 3068 & 7233 & 4499 \\
\hline$H(72 A)$ & 2493 & 5965 & 4380 \\
\hline$H(72 B)$ & 3374 & 6059 & 4697 \\
\hline$H(73 A)$ & 3441 & 5324 & 3853 \\
\hline$H(73 B)$ & 2705 & 5670 & 3396 \\
\hline$H(74 A)$ & 4176 & 6199 & 3671 \\
\hline$H(74 B)$ & 3544 & 6287 & 3020 \\
\hline
\end{tabular}


Table S18. Torsion angles [deg] for 6.

$$
\begin{aligned}
& N(5)-C r(2)-N a(1)-N(8) \\
& 106.01(18) \\
& \mathrm{N}(6)-\mathrm{Cr}(2)-\mathrm{Na}(1)-\mathrm{N}(8) \\
& 55 . \odot(3) \\
& \mathrm{N}(4)-\mathrm{Cr}(2)-\mathrm{Na}(1)-\mathrm{N}(8) \\
& \mathrm{O}(2)-\mathrm{Cr}(2)-\mathrm{Na}(1)-\mathrm{N}(8) \\
& -175.3(2) \\
& \mathrm{Na}(2)-\mathrm{Cr}(2)-\mathrm{Na}(1)-\mathrm{N}(8) \\
& -94.66(17) \\
& \mathrm{N}(8)-\mathrm{Cr}(2)-\mathrm{Na}(1)-\mathrm{N}(2) \\
& 59.25(15) \\
& \mathrm{N}(5)-\mathrm{Cr}(2)-\mathrm{Na}(1)-\mathrm{N}(2) \\
& -44.52(18) \\
& \mathrm{N}(6)-\mathrm{Cr}(2)-\mathrm{Na}(1)-\mathrm{N}(2) \\
& 61.49(16) \\
& 10.4(3) \\
& \mathrm{N}(4)-\mathrm{Cr}(2)-\mathrm{Na}(1)-\mathrm{N}(2) \\
& 140.15(18) \\
& \mathrm{O}(2)-\mathrm{Cr}(2)-\mathrm{Na}(1)-\mathrm{N}(2) \\
& -139.18(15) \\
& \mathrm{Na}(2)-\mathrm{Cr}(2)-\mathrm{Na}(1)-\mathrm{N}(2) \\
& \text { 14.72(12) } \\
& \mathrm{N}(8)-\mathrm{Cr}(2)-\mathrm{Na}(1)-\mathrm{N}(4) \\
& 175.3(2) \\
& \mathrm{N}(5)-\mathrm{Cr}(2)-\mathrm{Na}(1)-\mathrm{N}(4) \\
& -78.67(16) \\
& \mathrm{N}(6)-\mathrm{Cr}(2)-\mathrm{Na}(1)-\mathrm{N}(4) \\
& -129.7(3) \\
& \mathrm{O}(2)-\mathrm{Cr}(2)-\mathrm{Na}(1)-\mathrm{N}(4) \\
& 80.66(15) \\
& \mathrm{Na}(2)-\mathrm{Cr}(2)-\mathrm{Na}(1)-\mathrm{N}(4) \\
& -125.43(13) \\
& \mathrm{N}(8)-\mathrm{Cr}(2)-\mathrm{Na}(1)-\mathrm{N}(7) \\
& -11.21(17) \\
& \mathrm{N}(5)-\mathrm{Cr}(2)-\mathrm{Na}(1)-\mathrm{N}(7) \\
& 94.80(14) \\
& \mathrm{N}(6)-\mathrm{Cr}(2)-\mathrm{Na}(1)-\mathrm{N}(7) \\
& 43.8(3) \\
& \mathrm{N}(4)-\mathrm{Cr}(2)-\mathrm{Na}(1)-\mathrm{N}(7) \\
& 173.46(16) \\
& \mathrm{O}(2)-\mathrm{Cr}(2)-\mathrm{Na}(1)-\mathrm{N}(7) \\
& -105.87(13) \\
& \mathrm{Na}(2)-\mathrm{Cr}(2)-\mathrm{Na}(1)-\mathrm{N}(7) \\
& 48.03(10) \\
& \mathrm{N}(8)-\mathrm{Cr}(2)-\mathrm{Na}(1)-\mathrm{C}(3) \\
& -29.8(3) \\
& \mathrm{N}(5)-\mathrm{Cr}(2)-\mathrm{Na}(1)-\mathrm{C}(3) \\
& 76.2(3) \\
& \mathrm{N}(6)-\mathrm{Cr}(2)-\mathrm{Na}(1)-\mathrm{C}(3) \\
& 25.2(4)
\end{aligned}
$$




$$
\begin{aligned}
& \mathrm{N}(4)-\mathrm{Cr}(2)-\mathrm{Na}(1)-\mathrm{C}(3) \quad 154.9 \text { (3) } \\
& \mathrm{O}(2)-\mathrm{Cr}(2)-\mathrm{Na}(1)-\mathrm{C}(3) \quad-124.5 \text { (3) } \\
& \mathrm{Na}(2)-\mathrm{Cr}(2)-\mathrm{Na}(1)-\mathrm{C}(3) \quad 29.4 \text { (3) } \\
& \mathrm{N}(8)-\mathrm{Cr}(2)-\mathrm{Na}(1)-\mathrm{C}(39) \quad-151.48(19) \\
& \mathrm{N}(5)-\mathrm{Cr}(2)-\mathrm{Na}(1)-\mathrm{C}(39) \quad-45.47 \text { (15) } \\
& \mathrm{N}(6)-\mathrm{Cr}(2)-\mathrm{Na}(1)-\mathrm{C}(39) \quad-96.5(3) \\
& \mathrm{N}(4)-\mathrm{Cr}(2)-\mathrm{Na}(1)-\mathrm{C}(39) \quad 33.20(16) \\
& \mathrm{O}(2)-\mathrm{Cr}(2)-\mathrm{Na}(1)-\mathrm{C}(39) \quad 113.86(15) \\
& \mathrm{Na}(2)-\mathrm{Cr}(2)-\mathrm{Na}(1)-\mathrm{C}(39) \quad-92.23(12) \\
& \mathrm{N}(8)-\mathrm{Cr}(2)-\mathrm{Na}(1)-\mathrm{C}(54) \quad 123.1(2) \\
& \mathrm{N}(5)-\mathrm{Cr}(2)-\mathrm{Na}(1)-\mathrm{C}(54) \quad-130.87 \text { (16) } \\
& \mathrm{N}(6)-\mathrm{Cr}(2)-\mathrm{Na}(1)-\mathrm{C}(54) \quad 178.1(2) \\
& \mathrm{N}(4)-\mathrm{Cr}(2)-\mathrm{Na}(1)-\mathrm{C}(54) \quad-52.20(17) \\
& \mathrm{O}(2)-\mathrm{Cr}(2)-\mathrm{Na}(1)-\mathrm{C}(54) \quad 28.46(16) \\
& \mathrm{Na}(2)-\mathrm{Cr}(2)-\mathrm{Na}(1)-\mathrm{C}(54) \quad-177.63(14) \\
& \mathrm{N}(8)-\mathrm{Cr}(2)-\mathrm{Na}(1)-\mathrm{C}(4) \quad-96.3(4) \\
& \mathrm{N}(5)-\mathrm{Cr}(2)-\mathrm{Na}(1)-\mathrm{C}(4) \quad 9.7(4) \\
& \mathrm{N}(6)-\mathrm{Cr}(2)-\mathrm{Na}(1)-\mathrm{C}(4) \quad-41.4(5) \\
& \mathrm{N}(4)-\mathrm{Cr}(2)-\mathrm{Na}(1)-\mathrm{C}(4) \quad 88.3(4) \\
& \mathrm{O}(2)-\mathrm{Cr}(2)-\mathrm{Na}(1)-\mathrm{C}(4) \quad 169 . \odot(4) \\
& \mathrm{Na}(2)-\mathrm{Cr}(2)-\mathrm{Na}(1)-\mathrm{C}(4) \quad-37.1(4) \\
& \mathrm{N}(8)-\mathrm{Cr}(2)-\mathrm{Na}(1)-\mathrm{Cr}(1) \quad-18.96 \text { (14) } \\
& \mathrm{N}(5)-\mathrm{Cr}(2)-\mathrm{Na}(1)-\mathrm{Cr}(1) \quad 87.06(11) \\
& \mathrm{N}(6)-\mathrm{Cr}(2)-\mathrm{Na}(1)-\mathrm{Cr}(1) \quad 36 . \odot(2) \\
& \mathrm{N}(4)-\mathrm{Cr}(2)-\mathrm{Na}(1)-\mathrm{Cr}(1) \quad 165.72(14) \\
& \mathrm{O}(2)-\mathrm{Cr}(2)-\mathrm{Na}(1)-\mathrm{Cr}(1) \quad-113.62(9) \\
& \mathrm{Na}(2)-\mathrm{Cr}(2)-\mathrm{Na}(1)-\mathrm{Cr}(1) \quad 40.29(5) \\
& \mathrm{N}(8)-\mathrm{Cr}(2)-\mathrm{Na}(1)-\mathrm{Na}(2) \quad-59.25(15)
\end{aligned}
$$




$$
\begin{aligned}
& \mathrm{N}(5)-\mathrm{Cr}(2)-\mathrm{Na}(1)-\mathrm{Na}(2) \\
& 46.76(11) \\
& \mathrm{N}(6)-\mathrm{Cr}(2)-\mathrm{Na}(1)-\mathrm{Na}(2) \\
& -4.3(2) \\
& \mathrm{N}(4)-\mathrm{Cr}(2)-\mathrm{Na}(1)-\mathrm{Na}(2) \\
& 125.43(13) \\
& \mathrm{O}(2)-\mathrm{Cr}(2)-\mathrm{Na}(1)-\mathrm{Na}(2) \\
& \mathrm{N}(7)-\mathrm{Cr}(1)-\mathrm{Na}(1)-\mathrm{N}(8) \\
& \mathrm{N}(2)-\mathrm{Cr}(1)-\mathrm{Na}(1)-\mathrm{N}(8) \\
& \mathrm{N}(1)-\mathrm{Cr}(1)-\mathrm{Na}(1)-\mathrm{N}(8) \\
& \mathrm{N}(3)-\mathrm{Cr}(1)-\mathrm{Na}(1)-\mathrm{N}(8) \\
& \mathrm{O}(1)-\mathrm{Cr}(1)-\mathrm{Na}(1)-\mathrm{N}(8) \\
& \mathrm{Na}(2)-\mathrm{Cr}(1)-\mathrm{Na}(1)-\mathrm{N}(8) \\
& \mathrm{N}(7)-\mathrm{Cr}(1)-\mathrm{Na}(1)-\mathrm{N}(2) \\
& \mathrm{N}(1)-\mathrm{Cr}(1)-\mathrm{Na}(1)-\mathrm{N}(2) \\
& \mathrm{N}(3)-\mathrm{Cr}(1)-\mathrm{Na}(1)-\mathrm{N}(2) \\
& \mathrm{O}(1)-\mathrm{Cr}(1)-\mathrm{Na}(1)-\mathrm{N}(2) \\
& \mathrm{Na}(2)-\mathrm{Cr}(1)-\mathrm{Na}(1)-\mathrm{N}(2) \\
& \mathrm{N}(7)-\mathrm{Cr}(1)-\mathrm{Na}(1)-\mathrm{N}(4) \\
& \mathrm{N}(2)-\mathrm{Cr}(1)-\mathrm{Na}(1)-\mathrm{N}(4) \\
& \mathrm{N}(1)-\mathrm{Cr}(1)-\mathrm{Na}(1)-\mathrm{N}(4) \\
& \mathrm{N}(3)-\mathrm{Cr}(1)-\mathrm{Na}(1)-\mathrm{N}(4) \\
& \mathrm{O}(1)-\mathrm{Cr}(1)-\mathrm{Na}(1)-\mathrm{N}(4) \\
& \mathrm{Na}(2)-\mathrm{Cr}(1)-\mathrm{Na}(1)-\mathrm{N}(4) \\
& \mathrm{N}(2)-\mathrm{Cr}(1)-\mathrm{Na}(1)-\mathrm{N}(7) \\
& \mathrm{N}(1)-\mathrm{Cr}(1)-\mathrm{Na}(1)-\mathrm{N}(7) \\
& \mathrm{N}(3)-\mathrm{Cr}(1)-\mathrm{Na}(1)-\mathrm{N}(7) \\
& \mathrm{O}(1)-\mathrm{Cr}(1)-\mathrm{Na}(1)-\mathrm{N}(7) \\
& \mathrm{Na}(2)-\mathrm{Cr}(1)-\mathrm{Na}(1)-\mathrm{N}(7) \\
& \mathrm{N}(7)-\mathrm{Cr}(1)-\mathrm{Na}(1)-\mathrm{C}(3) \\
& \mathrm{N}(2)-\mathrm{Cr}(1)-\mathrm{Na}(1)-\mathrm{C}(3) \\
& \mathrm{N}(1)-\mathrm{Cr}(1)-\mathrm{Na}(1)-\mathrm{C}(3) \\
& -153.91(10) \\
& 0.87(17) \\
& -153.99(17) \\
& 124.27 \text { (14) } \\
& -97.69(14) \\
& 41.1(2) \\
& -56.74(10) \\
& \text { 154.9(2) } \\
& -81.74(17) \\
& 56.30(18) \\
& -164.9(2) \\
& 97.25(15) \\
& 25.59(19) \\
& -129.3(2) \\
& 149.00(18) \\
& -72.96(19) \\
& 65.9(3) \\
& -32.02(15) \\
& -154.9(2) \\
& \text { 123.41(18) } \\
& -98.56(18) \\
& 40.3(2) \\
& -57.61(15) \\
& -173.5(2) \\
& 31.69(18) \\
& -50.05(17)
\end{aligned}
$$




$$
\begin{aligned}
& \mathrm{N}(3)-\mathrm{Cr}(1)-\mathrm{Na}(1)-\mathrm{C}(3) \quad 87.99 \text { (17) } \\
& \mathrm{O}(1)-\mathrm{Cr}(1)-\mathrm{Na}(1)-\mathrm{C}(3) \quad-133.2(2) \\
& \mathrm{Na}(2)-\mathrm{Cr}(1)-\mathrm{Na}(1)-\mathrm{C}(3) \quad 128.94 \text { (14) } \\
& \mathrm{N}(7)-\mathrm{Cr}(1)-\mathrm{Na}(1)-\mathrm{C}(39) \quad 66.3(2) \\
& \mathrm{N}(2)-\mathrm{Cr}(1)-\mathrm{Na}(1)-\mathrm{C}(39) \quad-88.6(2) \\
& \mathrm{N}(1)-\mathrm{Cr}(1)-\mathrm{Na}(1)-\mathrm{C}(39) \quad-170.30(18) \\
& \mathrm{N}(3)-\mathrm{Cr}(1)-\mathrm{Na}(1)-\mathrm{C}(39) \quad-32.26(18) \\
& \mathrm{O}(1)-\mathrm{Cr}(1)-\mathrm{Na}(1)-\mathrm{C}(39) \quad 106.6(2) \\
& \mathrm{Na}(2)-\mathrm{Cr}(1)-\mathrm{Na}(1)-\mathrm{C}(39) \quad 8.68(14) \\
& \mathrm{N}(7)-\mathrm{Cr}(1)-\mathrm{Na}(1)-\mathrm{C}(54) \quad-106.6(2) \\
& \mathrm{N}(2)-\mathrm{Cr}(1)-\mathrm{Na}(1)-\mathrm{C}(54) \quad 98.5(2) \\
& \mathrm{N}(1)-\mathrm{Cr}(1)-\mathrm{Na}(1)-\mathrm{C}(54) \quad 16.8(2) \\
& \mathrm{N}(3)-\mathrm{Cr}(1)-\mathrm{Na}(1)-\mathrm{C}(54) \quad 154.8(2) \\
& 0(1)-\mathrm{Cr}(1)-\mathrm{Na}(1)-\mathrm{C}(54) \quad-66.3(3) \\
& \mathrm{Na}(2)-\mathrm{Cr}(1)-\mathrm{Na}(1)-\mathrm{C}(54) \quad-164.21 \text { (19) } \\
& \mathrm{N}(7)-\mathrm{Cr}(1)-\mathrm{Na}(1)-\mathrm{C}(4) \quad 175.61 \text { (19) } \\
& \mathrm{N}(2)-\mathrm{Cr}(1)-\mathrm{Na}(1)-\mathrm{C}(4) \quad 20.75 \text { (17) } \\
& \mathrm{N}(1)-\mathrm{Cr}(1)-\mathrm{Na}(1)-\mathrm{C}(4) \quad-60.98(15) \\
& \mathrm{N}(3)-\mathrm{Cr}(1)-\mathrm{Na}(1)-\mathrm{C}(4) \quad 77.05 \text { (15) } \\
& 0(1)-\mathrm{Cr}(1)-\mathrm{Na}(1)-\mathrm{C}(4) \quad-144.1(2) \\
& \mathrm{Na}(2)-\mathrm{Cr}(1)-\mathrm{Na}(1)-\mathrm{C}(4) \quad 118.00(12) \\
& \mathrm{N}(7)-\mathrm{Cr}(1)-\mathrm{Na}(1)-\mathrm{Cr}(2) \quad 12.71 \text { (14) } \\
& \mathrm{N}(2)-\mathrm{Cr}(1)-\mathrm{Na}(1)-\mathrm{Cr}(2) \quad-142.15 \text { (15) } \\
& \mathrm{N}(1)-\mathrm{Cr}(1)-\mathrm{Na}(1)-\mathrm{Cr}(2) \quad 136.11(12) \\
& \mathrm{N}(3)-\mathrm{Cr}(1)-\mathrm{Na}(1)-\mathrm{Cr}(2) \quad-85.85(12) \\
& 0(1)-\mathrm{Cr}(1)-\mathrm{Na}(1)-\mathrm{Cr}(2) \quad 53.0(2) \\
& \mathrm{Na}(2)-\mathrm{Cr}(1)-\mathrm{Na}(1)-\mathrm{Cr}(2) \quad-44.90(6) \\
& \mathrm{N}(7)-\mathrm{Cr}(1)-\mathrm{Na}(1)-\mathrm{Na}(2) \quad 57.61 \text { (15) }
\end{aligned}
$$




$$
\begin{aligned}
& \mathrm{N}(2)-\mathrm{Cr}(1)-\mathrm{Na}(1)-\mathrm{Na}(2) \\
& -97.25(15) \\
& \mathrm{N}(1)-\mathrm{Cr}(1)-\mathrm{Na}(1)-\mathrm{Na}(2) \\
& -178.98(12) \\
& \mathrm{N}(3)-\mathrm{Cr}(1)-\mathrm{Na}(1)-\mathrm{Na}(2) \\
& -40.95(12) \\
& \mathrm{O}(1)-\mathrm{Cr}(1)-\mathrm{Na}(1)-\mathrm{Na}(2) \\
& 97.87(19) \\
& \mathrm{N}(2)-\mathrm{Cr}(1)-\mathrm{Na}(2)-\mathrm{N}(7) \\
& 108.68(17) \\
& \mathrm{N}(1)-\mathrm{Cr}(1)-\mathrm{Na}(2)-\mathrm{N}(7) \\
& 59.1(3) \\
& \mathrm{N}(3)-\mathrm{Cr}(1)-\mathrm{Na}(2)-\mathrm{N}(7) \\
& O(1)-\mathrm{Cr}(1)-\mathrm{Na}(2)-\mathrm{N}(7) \\
& -172.20(19) \\
& -92.25(17) \\
& \mathrm{Na}(1)-\mathrm{Cr}(1)-\mathrm{Na}(2)-\mathrm{N}(7) \\
& 56.99(14) \\
& \mathrm{N}(7)-\mathrm{Cr}(1)-\mathrm{Na}(2)-\mathrm{N}(3) \\
& 172.20(19) \\
& \mathrm{N}(2)-\mathrm{Cr}(1)-\mathrm{Na}(2)-\mathrm{N}(3) \\
& \mathrm{N}(1)-\mathrm{Cr}(1)-\mathrm{Na}(2)-\mathrm{N}(3) \\
& O(1)-\mathrm{Cr}(1)-\mathrm{Na}(2)-\mathrm{N}(3) \\
& \mathrm{Na}(1)-\mathrm{Cr}(1)-\mathrm{Na}(2)-\mathrm{N}(3) \\
& \mathrm{N}(7)-\mathrm{Cr}(1)-\mathrm{Na}(2)-\mathrm{N}(5) \\
& \mathrm{N}(2)-\mathrm{Cr}(1)-\mathrm{Na}(2)-\mathrm{N}(5) \\
& \mathrm{N}(1)-\mathrm{Cr}(1)-\mathrm{Na}(2)-\mathrm{N}(5) \\
& \mathrm{N}(3)-\mathrm{Cr}(1)-\mathrm{Na}(2)-\mathrm{N}(5) \\
& \mathrm{O}(1)-\mathrm{Cr}(1)-\mathrm{Na}(2)-\mathrm{N}(5) \\
& \mathrm{Na}(1)-\mathrm{Cr}(1)-\mathrm{Na}(2)-\mathrm{N}(5) \\
& N(7)-\mathrm{Cr}(1)-\mathrm{Na}(2)-\mathrm{C}(44) \\
& N(2)-C r(1)-N a(2)-C(44) \\
& N(1)-\mathrm{Cr}(1)-\mathrm{Na}(2)-\mathrm{C}(44) \\
& N(3)-\mathrm{Cr}(1)-\mathrm{Na}(2)-\mathrm{C}(44) \\
& \mathrm{O}(1)-\mathrm{Cr}(1)-\mathrm{Na}(2)-\mathrm{C}(44) \\
& \mathrm{Na}(1)-\mathrm{Cr}(1)-\mathrm{Na}(2)-\mathrm{C}(44) \\
& N(7)-\mathrm{Cr}(1)-\mathrm{Na}(2)-\mathrm{C}(8) \\
& N(2)-C r(1)-N a(2)-C(8) \\
& \mathrm{N}(1)-\mathrm{Cr}(1)-\mathrm{Na}(2)-\mathrm{C}(8) \\
& -79.12(16) \\
& -128.7(3) \\
& 79.95(15) \\
& -130.82(13) \\
& -38.08(18) \\
& 70.6 \odot(16) \\
& 21.1(3) \\
& 149.72(19) \\
& -130.33(15) \\
& \text { 18. 91(12) } \\
& -18.6(3) \\
& 90.1(3) \\
& 40.5(4) \\
& 169.2(3) \\
& -110.9(3) \\
& 38.4(2) \\
& -154.46(19) \\
& -45.78(16) \\
& -95.3(3)
\end{aligned}
$$




$$
\begin{aligned}
& \mathrm{N}(3)-\mathrm{Cr}(1)-\mathrm{Na}(2)-\mathrm{C}(8) \quad 33.34 \text { (17) } \\
& \mathrm{O}(1)-\mathrm{Cr}(1)-\mathrm{Na}(2)-\mathrm{C}(8) \quad 113.29(15) \\
& \mathrm{Na}(1)-\mathrm{Cr}(1)-\mathrm{Na}(2)-\mathrm{C}(8) \quad-97.47(13) \\
& \mathrm{N}(7)-\mathrm{Cr}(1)-\mathrm{Na}(2)-\mathrm{N}(8) \quad-8.46(16) \\
& \mathrm{N}(2)-\mathrm{Cr}(1)-\mathrm{Na}(2)-\mathrm{N}(8) \quad 100.23(13) \\
& \mathrm{N}(1)-\mathrm{Cr}(1)-\mathrm{Na}(2)-\mathrm{N}(8) \quad 50.7 \text { (3) } \\
& \mathrm{N}(3)-\mathrm{Cr}(1)-\mathrm{Na}(2)-\mathrm{N}(8) \quad 179.35(16) \\
& \mathrm{O}(1)-\mathrm{Cr}(1)-\mathrm{Na}(2)-\mathrm{N}(8) \quad-100.71(12) \\
& \mathrm{Na}(1)-\mathrm{Cr}(1)-\mathrm{Na}(2)-\mathrm{N}(8) \quad 48.53(9) \\
& \mathrm{N}(7)-\mathrm{Cr}(1)-\mathrm{Na}(2)-\mathrm{C}(43) \quad-80.2(5) \\
& \mathrm{N}(2)-\mathrm{Cr}(1)-\mathrm{Na}(2)-\mathrm{C}(43) \quad 28.5(5) \\
& \mathrm{N}(1)-\mathrm{Cr}(1)-\mathrm{Na}(2)-\mathrm{C}(43) \quad-21.1(6) \\
& \mathrm{N}(3)-\mathrm{Cr}(1)-\mathrm{Na}(2)-\mathrm{C}(43) \quad 107.6(5) \\
& 0(1)-\mathrm{Cr}(1)-\mathrm{Na}(2)-\mathrm{C}(43) \quad-172.5(5) \\
& \mathrm{Na}(1)-\mathrm{Cr}(1)-\mathrm{Na}(2)-\mathrm{C}(43) \quad-23.2(5) \\
& \mathrm{N}(7)-\mathrm{Cr}(1)-\mathrm{Na}(2)-\mathrm{C}(32) \quad 122.8(2) \\
& \mathrm{N}(2)-\mathrm{Cr}(1)-\mathrm{Na}(2)-\mathrm{C}(32) \quad-128.49 \text { (18) } \\
& \mathrm{N}(1)-\mathrm{Cr}(1)-\mathrm{Na}(2)-\mathrm{C}(32) \quad-178 . \odot \text { (3) } \\
& \mathrm{N}(3)-\mathrm{Cr}(1)-\mathrm{Na}(2)-\mathrm{C}(32) \quad-49.37 \text { (19) } \\
& 0(1)-\mathrm{Cr}(1)-\mathrm{Na}(2)-\mathrm{C}(32) \quad 30.58(18) \\
& \mathrm{Na}(1)-\mathrm{Cr}(1)-\mathrm{Na}(2)-\mathrm{C}(32) \quad 179.82(16) \\
& \mathrm{N}(7)-\mathrm{Cr}(1)-\mathrm{Na}(2)-\mathrm{C}(40) \quad-66.57(17) \\
& \mathrm{N}(2)-\mathrm{Cr}(1)-\mathrm{Na}(2)-\mathrm{C}(40) \quad 42.11(15) \\
& \mathrm{N}(1)-\mathrm{Cr}(1)-\mathrm{Na}(2)-\mathrm{C}(40) \quad-7.4 \text { (3) } \\
& \mathrm{N}(3)-\mathrm{Cr}(1)-\mathrm{Na}(2)-\mathrm{C}(40) \quad 121.24 \text { (17) } \\
& 0(1)-\mathrm{Cr}(1)-\mathrm{Na}(2)-\mathrm{C}(40) \quad-158.82(13) \\
& \mathrm{Na}(1)-\mathrm{Cr}(1)-\mathrm{Na}(2)-\mathrm{C}(40) \quad-9.58(11) \\
& \mathrm{N}(7)-\mathrm{Cr}(1)-\mathrm{Na}(2)-\mathrm{Cr}(2) \quad-16.78(14)
\end{aligned}
$$




$$
\begin{aligned}
& \mathrm{N}(2)-\mathrm{Cr}(1)-\mathrm{Na}(2)-\mathrm{Cr}(2) \\
& 91.90(11) \\
& \mathrm{N}(1)-\mathrm{Cr}(1)-\mathrm{Na}(2)-\mathrm{Cr}(2) \\
& 42.4(3) \\
& \mathrm{N}(3)-\mathrm{Cr}(1)-\mathrm{Na}(2)-\mathrm{Cr}(2) \\
& 171.02(14) \\
& \mathrm{O}(1)-\mathrm{Cr}(1)-\mathrm{Na}(2)-\mathrm{Cr}(2) \\
& \mathrm{Na}(1)-\mathrm{Cr}(1)-\mathrm{Na}(2)-\mathrm{Cr}(2) \\
& \mathrm{N}(7)-\mathrm{Cr}(1)-\mathrm{Na}(2)-\mathrm{Na}(1) \\
& \mathrm{N}(2)-\mathrm{Cr}(1)-\mathrm{Na}(2)-\mathrm{Na}(1) \\
& \mathrm{N}(1)-\mathrm{Cr}(1)-\mathrm{Na}(2)-\mathrm{Na}(1) \\
& \mathrm{N}(3)-\mathrm{Cr}(1)-\mathrm{Na}(2)-\mathrm{Na}(1) \\
& \mathrm{O}(1)-\mathrm{Cr}(1)-\mathrm{Na}(2)-\mathrm{Na}(1) \\
& \mathrm{N}(8)-\mathrm{Cr}(2)-\mathrm{Na}(2)-\mathrm{N}(7) \\
& \mathrm{N}(5)-\mathrm{Cr}(2)-\mathrm{Na}(2)-\mathrm{N}(7) \\
& N(6)-\mathrm{Cr}(2)-\mathrm{Na}(2)-\mathrm{N}(7) \\
& \mathrm{N}(4)-\mathrm{Cr}(2)-\mathrm{Na}(2)-\mathrm{N}(7) \\
& \mathrm{O}(2)-\mathrm{Cr}(2)-\mathrm{Na}(2)-\mathrm{N}(7) \\
& \mathrm{Na}(1)-\mathrm{Cr}(2)-\mathrm{Na}(2)-\mathrm{N}(7) \\
& N(8)-\mathrm{Cr}(2)-\mathrm{Na}(2)-\mathrm{N}(3) \\
& \mathrm{N}(5)-\mathrm{Cr}(2)-\mathrm{Na}(2)-\mathrm{N}(3) \\
& N(6)-\mathrm{Cr}(2)-\mathrm{Na}(2)-\mathrm{N}(3) \\
& \mathrm{N}(4)-\mathrm{Cr}(2)-\mathrm{Na}(2)-\mathrm{N}(3) \\
& \mathrm{O}(2)-\mathrm{Cr}(2)-\mathrm{Na}(2)-\mathrm{N}(3) \\
& \mathrm{Na}(1)-\mathrm{Cr}(2)-\mathrm{Na}(2)-\mathrm{N}(3) \\
& \mathrm{N}(8)-\mathrm{Cr}(2)-\mathrm{Na}(2)-\mathrm{N}(5) \\
& N(6)-C r(2)-\mathrm{Na}(2)-N(5) \\
& N(4)-\mathrm{Cr}(2)-\mathrm{Na}(2)-\mathrm{N}(5) \\
& \mathrm{O}(2)-\mathrm{Cr}(2)-\mathrm{Na}(2)-\mathrm{N}(5) \\
& \mathrm{Na}(1)-\mathrm{Cr}(2)-\mathrm{Na}(2)-\mathrm{N}(5) \\
& \mathrm{N}(8)-\mathrm{Cr}(2)-\mathrm{Na}(2)-\mathrm{C}(44) \\
& N(5)-C r(2)-N a(2)-C(44) \\
& -109.03(10) \\
& 40.21(5) \\
& -56.99(14) \\
& \text { 51. 69(11) } \\
& 2.2(2) \\
& 130.82(13) \\
& -149.24(10) \\
& 3.35(17) \\
& -156.75(18) \\
& 123.77(14) \\
& -97.49(14) \\
& 35.5(3) \\
& -54.20(10) \\
& 22.8(2) \\
& -137.3(2) \\
& 143.21(18) \\
& -78.05(19) \\
& 55.0(3) \\
& -34.75(15) \\
& 160.1(2) \\
& -79.48(17) \\
& 59.25(17) \\
& -167.7(3) \\
& 102.55(15) \\
& -166.67(19) \\
& 33.23(18)
\end{aligned}
$$




$$
\begin{aligned}
& \mathrm{N}(6)-\mathrm{Cr}(2)-\mathrm{Na}(2)-\mathrm{C}(44) \quad-46.25(16) \\
& \mathrm{N}(4)-\mathrm{Cr}(2)-\mathrm{Na}(2)-\mathrm{C}(44) \quad 92.49(16) \\
& \mathrm{O}(2)-\mathrm{Cr}(2)-\mathrm{Na}(2)-\mathrm{C}(44) \quad-134.5(2) \\
& \mathrm{Na}(1)-\mathrm{Cr}(2)-\mathrm{Na}(2)-\mathrm{C}(44) \quad 135.79 \text { (13) } \\
& \mathrm{N}(8)-\mathrm{Cr}(2)-\mathrm{Na}(2)-\mathrm{C}(8) \quad 65.7(2) \\
& \mathrm{N}(5)-\mathrm{Cr}(2)-\mathrm{Na}(2)-\mathrm{C}(8) \quad-94.4(2) \\
& \mathrm{N}(6)-\mathrm{Cr}(2)-\mathrm{Na}(2)-\mathrm{C}(8) \quad-173.92(19) \\
& \mathrm{N}(4)-\mathrm{Cr}(2)-\mathrm{Na}(2)-\mathrm{C}(8) \quad-35.18(19) \\
& \mathrm{O}(2)-\mathrm{Cr}(2)-\mathrm{Na}(2)-\mathrm{C}(8) \quad 97.8(3) \\
& \mathrm{Na}(1)-\mathrm{Cr}(2)-\mathrm{Na}(2)-\mathrm{C}(8) \quad 8.11(16) \\
& \mathrm{N}(5)-\mathrm{Cr}(2)-\mathrm{Na}(2)-\mathrm{N}(8) \quad-160.1 \text { (2) } \\
& \mathrm{N}(6)-\mathrm{Cr}(2)-\mathrm{Na}(2)-\mathrm{N}(8) \quad 120.42(17) \\
& \mathrm{N}(4)-\mathrm{Cr}(2)-\mathrm{Na}(2)-\mathrm{N}(8) \quad-100.84 \text { (18) } \\
& \mathrm{O}(2)-\mathrm{Cr}(2)-\mathrm{Na}(2)-\mathrm{N}(8) \quad 32.2(3) \\
& \mathrm{Na}(1)-\mathrm{Cr}(2)-\mathrm{Na}(2)-\mathrm{N}(8) \quad-57.55(15) \\
& \mathrm{N}(8)-\mathrm{Cr}(2)-\mathrm{Na}(2)-\mathrm{C}(43) \quad-177.34(18) \\
& \mathrm{N}(5)-\mathrm{Cr}(2)-\mathrm{Na}(2)-\mathrm{C}(43) \quad 22.56(17) \\
& \mathrm{N}(6)-\mathrm{Cr}(2)-\mathrm{Na}(2)-\mathrm{C}(43) \quad-56.92(14) \\
& \mathrm{N}(4)-\mathrm{Cr}(2)-\mathrm{Na}(2)-\mathrm{C}(43) \quad 81.82(15) \\
& 0(2)-\mathrm{Cr}(2)-\mathrm{Na}(2)-\mathrm{C}(43) \quad-145.2(2) \\
& \mathrm{Na}(1)-\mathrm{Cr}(2)-\mathrm{Na}(2)-\mathrm{C}(43) \quad 125.12(12) \\
& \mathrm{N}(8)-\mathrm{Cr}(2)-\mathrm{Na}(2)-\mathrm{C}(32) \quad-102.6(2) \\
& \mathrm{N}(5)-\mathrm{Cr}(2)-\mathrm{Na}(2)-\mathrm{C}(32) \quad 97.3(2) \\
& \mathrm{N}(6)-\mathrm{Cr}(2)-\mathrm{Na}(2)-\mathrm{C}(32) \quad 17.8(2) \\
& \mathrm{N}(4)-\mathrm{Cr}(2)-\mathrm{Na}(2)-\mathrm{C}(32) \quad 156.5(2) \\
& \mathrm{O}(2)-\mathrm{Cr}(2)-\mathrm{Na}(2)-\mathrm{C}(32) \quad-70.5(3) \\
& \mathrm{Na}(1)-\mathrm{Cr}(2)-\mathrm{Na}(2)-\mathrm{C}(32) \quad-160.2(2) \\
& \mathrm{N}(8)-\mathrm{Cr}(2)-\mathrm{Na}(2)-\mathrm{Cr}(1) \quad 14.55(14)
\end{aligned}
$$




$$
\begin{aligned}
& \mathrm{N}(5)-\mathrm{Cr}(2)-\mathrm{Na}(2)-\mathrm{Cr}(1) \quad-145.55(16) \\
& \mathrm{N}(6)-\mathrm{Cr}(2)-\mathrm{Na}(2)-\mathrm{Cr}(1) \quad 134.97(11) \\
& \mathrm{N}(4)-\mathrm{Cr}(2)-\mathrm{Na}(2)-\mathrm{Cr}(1) \quad-86.29(11) \\
& \mathrm{O}(2)-\mathrm{Cr}(2)-\mathrm{Na}(2)-\mathrm{Cr}(1) \quad 46.7(2) \\
& \mathrm{Na}(1)-\mathrm{Cr}(2)-\mathrm{Na}(2)-\mathrm{Cr}(1) \quad-43.0 \odot(6) \\
& \mathrm{N}(8)-\mathrm{Cr}(2)-\mathrm{Na}(2)-\mathrm{C}(40) \quad 130.33(18) \\
& \mathrm{N}(5)-\mathrm{Cr}(2)-\mathrm{Na}(2)-\mathrm{C}(4 \odot) \quad-29.77(17) \\
& \mathrm{N}(6)-\mathrm{Cr}(2)-\mathrm{Na}(2)-\mathrm{C}(40) \quad-109.25(14) \\
& \mathrm{N}(4)-\mathrm{Cr}(2)-\mathrm{Na}(2)-\mathrm{C}(40) \quad 29.49(15) \\
& \mathrm{O}(2)-\mathrm{Cr}(2)-\mathrm{Na}(2)-\mathrm{C}(40) \quad 162.5(2) \\
& \begin{array}{ll}
\mathrm{Na}(1)-\mathrm{Cr}(2)-\mathrm{Na}(2)-\mathrm{C}(40) & 72.79(11)
\end{array} \\
& \mathrm{N}(8)-\mathrm{Cr}(2)-\mathrm{Na}(2)-\mathrm{Na}(1) \quad 57.55(15) \\
& \mathrm{N}(5)-\mathrm{Cr}(2)-\mathrm{Na}(2)-\mathrm{Na}(1) \quad-102.55(15) \\
& \mathrm{N}(6)-\mathrm{Cr}(2)-\mathrm{Na}(2)-\mathrm{Na}(1) \quad 177.97(11) \\
& \mathrm{N}(4)-\mathrm{Cr}(2)-\mathrm{Na}(2)-\mathrm{Na}(1) \quad-43.30(11) \\
& 0(2)-\mathrm{Cr}(2)-\mathrm{Na}(2)-\mathrm{Na}(1) \quad 89.7(2) \\
& \mathrm{N}(8)-\mathrm{Na}(1)-\mathrm{Na}(2)-\mathrm{N}(7) \quad 41.15(16) \\
& \mathrm{N}(2)-\mathrm{Na}(1)-\mathrm{Na}(2)-\mathrm{N}(7) \quad-84.71(16) \\
& \mathrm{N}(4)-\mathrm{Na}(1)-\mathrm{Na}(2)-\mathrm{N}(7) \quad 113.84(16) \\
& \mathrm{C}(3)-\mathrm{Na}(1)-\mathrm{Na}(2)-\mathrm{N}(7) \quad-84.82(17) \\
& \begin{array}{ll}
\mathrm{C}(39)-\mathrm{Na}(1)-\mathrm{Na}(2)-\mathrm{N}(7) & 143.57(17)
\end{array} \\
& \mathrm{C}(54)-\mathrm{Na}(1)-\mathrm{Na}(2)-\mathrm{N}(7) \quad 90.3(6) \\
& \mathrm{C}(4)-\mathrm{Na}(1)-\mathrm{Na}(2)-\mathrm{N}(7) \quad-109.43(17) \\
& \begin{array}{ll}
\mathrm{Cr}(2)-\mathrm{Na}(1)-\mathrm{Na}(2)-\mathrm{N}(7) & 81.40(13)
\end{array} \\
& \mathrm{Cr}(1)-\mathrm{Na}(1)-\mathrm{Na}(2)-\mathrm{N}(7) \quad-43.46(12) \\
& \mathrm{N}(8)-\mathrm{Na}(1)-\mathrm{Na}(2)-\mathrm{N}(3) \quad 115.14(15) \\
& \mathrm{N}(2)-\mathrm{Na}(1)-\mathrm{Na}(2)-\mathrm{N}(3) \quad-10.72(13) \\
& \mathrm{N}(4)-\mathrm{Na}(1)-\mathrm{Na}(2)-\mathrm{N}(3) \quad-172.17(13) \\
& \mathrm{N}(7)-\mathrm{Na}(1)-\mathrm{Na}(2)-\mathrm{N}(3) \quad 73.99(15)
\end{aligned}
$$




$$
\begin{aligned}
& \mathrm{C}(3)-\mathrm{Na}(1)-\mathrm{Na}(2)-\mathrm{N}(3) \quad-10.84 \text { (15) } \\
& \mathrm{C}(39)-\mathrm{Na}(1)-\mathrm{Na}(2)-\mathrm{N}(3) \quad-142.45 \text { (14) } \\
& \mathrm{C}(54)-\mathrm{Na}(1)-\mathrm{Na}(2)-\mathrm{N}(3) \quad 164.3(5) \\
& \mathrm{C}(4)-\mathrm{Na}(1)-\mathrm{Na}(2)-\mathrm{N}(3) \quad-35.44(15) \\
& \mathrm{Cr}(2)-\mathrm{Na}(1)-\mathrm{Na}(2)-\mathrm{N}(3) \quad 155.39(11) \\
& \mathrm{Cr}(1)-\mathrm{Na}(1)-\mathrm{Na}(2)-\mathrm{N}(3) \quad 30.52(9) \\
& \mathrm{N}(8)-\mathrm{Na}(1)-\mathrm{Na}(2)-\mathrm{N}(5) \quad-77.25(14) \\
& \mathrm{N}(2)-\mathrm{Na}(1)-\mathrm{Na}(2)-\mathrm{N}(5) \quad 156.89 \text { (15) } \\
& \mathrm{N}(4)-\mathrm{Na}(1)-\mathrm{Na}(2)-\mathrm{N}(5) \quad-4.56 \text { (13) } \\
& \mathrm{N}(7)-\mathrm{Na}(1)-\mathrm{Na}(2)-\mathrm{N}(5) \quad-118.40(16) \\
& \mathrm{C}(3)-\mathrm{Na}(1)-\mathrm{Na}(2)-\mathrm{N}(5) \quad 156.77 \text { (15) } \\
& \mathrm{C}(39)-\mathrm{Na}(1)-\mathrm{Na}(2)-\mathrm{N}(5) \quad 25.16 \text { (14) } \\
& \mathrm{C}(54)-\mathrm{Na}(1)-\mathrm{Na}(2)-\mathrm{N}(5) \quad-28.1 \text { (6) } \\
& \mathrm{C}(4)-\mathrm{Na}(1)-\mathrm{Na}(2)-\mathrm{N}(5) \quad 132.17 \text { (15) } \\
& \operatorname{Cr}(2)-\mathrm{Na}(1)-\mathrm{Na}(2)-\mathrm{N}(5) \quad-37.00(9) \\
& \mathrm{Cr}(1)-\mathrm{Na}(1)-\mathrm{Na}(2)-\mathrm{N}(5) \quad-161.87 \text { (12) } \\
& \mathrm{N}(8)-\mathrm{Na}(1)-\mathrm{Na}(2)-\mathrm{C}(44) \quad-75.44(16) \\
& \mathrm{N}(2)-\mathrm{Na}(1)-\mathrm{Na}(2)-\mathrm{C}(44) \quad 158.70 \text { (15) } \\
& \mathrm{N}(4)-\mathrm{Na}(1)-\mathrm{Na}(2)-\mathrm{C}(44) \quad-2.75 \text { (14) } \\
& \mathrm{N}(7)-\mathrm{Na}(1)-\mathrm{Na}(2)-\mathrm{C}(44) \quad-116.59(17) \\
& \mathrm{C}(3)-\mathrm{Na}(1)-\mathrm{Na}(2)-\mathrm{C}(44) \quad 158.58(16) \\
& \mathrm{C}(39)-\mathrm{Na}(1)-\mathrm{Na}(2)-\mathrm{C}(44) \quad 26.97(15) \\
& \mathrm{C}(54)-\mathrm{Na}(1)-\mathrm{Na}(2)-\mathrm{C}(44) \quad-26.2(6) \\
& \mathrm{C}(4)-\mathrm{Na}(1)-\mathrm{Na}(2)-\mathrm{C}(44) \quad 133.98(16) \\
& \mathrm{Cr}(2)-\mathrm{Na}(1)-\mathrm{Na}(2)-\mathrm{C}(44) \quad-35.19 \text { (11) } \\
& \mathrm{Cr}(1)-\mathrm{Na}(1)-\mathrm{Na}(2)-\mathrm{C}(44) \quad-160.06 \text { (13) } \\
& \mathrm{N}(8)-\mathrm{Na}(1)-\mathrm{Na}(2)-\mathrm{C}(8) \quad 145.95 \text { (16) } \\
& \mathrm{N}(2)-\mathrm{Na}(1)-\mathrm{Na}(2)-\mathrm{C}(8) \quad 20.09 \text { (14) }
\end{aligned}
$$




$$
\begin{aligned}
& \mathrm{N}(4)-\mathrm{Na}(1)-\mathrm{Na}(2)-\mathrm{C}(8) \\
& -141.36(14) \\
& \mathrm{N}(7)-\mathrm{Na}(1)-\mathrm{Na}(2)-\mathrm{C}(8) \\
& 104.80(16) \\
& \mathrm{C}(3)-\mathrm{Na}(1)-\mathrm{Na}(2)-\mathrm{C}(8) \\
& 19.98(16) \\
& \mathrm{C}(39)-\mathrm{Na}(1)-\mathrm{Na}(2)-\mathrm{C}(8) \\
& -111.64(15) \\
& \mathrm{C}(54)-\mathrm{Na}(1)-\mathrm{Na}(2)-\mathrm{C}(8) \\
& \mathrm{C}(4)-\mathrm{Na}(1)-\mathrm{Na}(2)-\mathrm{C}(8) \\
& -164.9(5) \\
& -4.63(16) \\
& \mathrm{Cr}(2)-\mathrm{Na}(1)-\mathrm{Na}(2)-\mathrm{C}(8) \\
& \mathrm{Cr}(1)-\mathrm{Na}(1)-\mathrm{Na}(2)-\mathrm{C}(8) \\
& -173.80(12) \\
& 61.34(11) \\
& N(2)-\mathrm{Na}(1)-\mathrm{Na}(2)-\mathrm{N}(8) \\
& \mathrm{N}(4)-\mathrm{Na}(1)-\mathrm{Na}(2)-\mathrm{N}(8) \\
& \mathrm{N}(7)-\mathrm{Na}(1)-\mathrm{Na}(2)-\mathrm{N}(8) \\
& \mathrm{C}(3)-\mathrm{Na}(1)-\mathrm{Na}(2)-\mathrm{N}(8) \\
& \mathrm{C}(39)-\mathrm{Na}(1)-\mathrm{Na}(2)-\mathrm{N}(8) \\
& \mathrm{C}(54)-\mathrm{Na}(1)-\mathrm{Na}(2)-\mathrm{N}(8) \\
& \mathrm{C}(4)-\mathrm{Na}(1)-\mathrm{Na}(2)-\mathrm{N}(8) \\
& \mathrm{Cr}(2)-\mathrm{Na}(1)-\mathrm{Na}(2)-\mathrm{N}(8) \\
& \mathrm{Cr}(1)-\mathrm{Na}(1)-\mathrm{Na}(2)-\mathrm{N}(8) \\
& \mathrm{N}(8)-\mathrm{Na}(1)-\mathrm{Na}(2)-\mathrm{C}(43) \\
& \mathrm{N}(2)-\mathrm{Na}(1)-\mathrm{Na}(2)-\mathrm{C}(43) \\
& \mathrm{N}(4)-\mathrm{Na}(1)-\mathrm{Na}(2)-\mathrm{C}(43) \\
& \mathrm{N}(7)-\mathrm{Na}(1)-\mathrm{Na}(2)-\mathrm{C}(43) \\
& \mathrm{C}(3)-\mathrm{Na}(1)-\mathrm{Na}(2)-\mathrm{C}(43) \\
& \mathrm{C}(39)-\mathrm{Na}(1)-\mathrm{Na}(2)-\mathrm{C}(43) \\
& \mathrm{C}(54)-\mathrm{Na}(1)-\mathrm{Na}(2)-\mathrm{C}(43) \\
& \mathrm{C}(4)-\mathrm{Na}(1)-\mathrm{Na}(2)-\mathrm{C}(43) \\
& \mathrm{Cr}(2)-\mathrm{Na}(1)-\mathrm{Na}(2)-\mathrm{C}(43) \\
& \mathrm{Cr}(1)-\mathrm{Na}(1)-\mathrm{Na}(2)-\mathrm{C}(43) \\
& \mathrm{N}(8)-\mathrm{Na}(1)-\mathrm{Na}(2)-\mathrm{C}(32) \\
& \mathrm{N}(2)-\mathrm{Na}(1)-\mathrm{Na}(2)-\mathrm{C}(32) \\
& -125.86(16) \\
& 72.68(14) \\
& -41.15(16) \\
& -125.98(17) \\
& 102.41(15) \\
& 49.2(5) \\
& -150.58(17) \\
& 40.25 \text { (11) } \\
& -84.62(12) \\
& -100.94(16) \\
& 133.20(16) \\
& -28.25(15) \\
& -142.09(18) \\
& \text { 133. } 08(16) \\
& \text { 1. } 47(16) \\
& -51.7(6) \\
& \text { 108. 48(16) } \\
& -60.69(12) \\
& 174.44(13) \\
& 84.0(6) \\
& -41.8(5)
\end{aligned}
$$




$$
\begin{aligned}
& \mathrm{N}(4)-\mathrm{Na}(1)-\mathrm{Na}(2)-\mathrm{C}(32) \\
& 156.7(5) \\
& \mathrm{N}(7)-\mathrm{Na}(1)-\mathrm{Na}(2)-\mathrm{C}(32) \\
& 42.9(5) \\
& \mathrm{C}(3)-\mathrm{Na}(1)-\mathrm{Na}(2)-\mathrm{C}(32) \\
& -42 . \odot(6) \\
& \mathrm{C}(39)-\mathrm{Na}(1)-\mathrm{Na}(2)-\mathrm{C}(32) \\
& -173.6(5) \\
& \mathrm{C}(54)-\mathrm{Na}(1)-\mathrm{Na}(2)-\mathrm{C}(32) \\
& 133.2(7) \\
& \mathrm{C}(4)-\mathrm{Na}(1)-\mathrm{Na}(2)-\mathrm{C}(32) \\
& -66.6(6) \\
& \mathrm{Cr}(2)-\mathrm{Na}(1)-\mathrm{Na}(2)-\mathrm{C}(32) \\
& 124.3(5) \\
& \mathrm{Cr}(1)-\mathrm{Na}(1)-\mathrm{Na}(2)-\mathrm{C}(32) \\
& -0.6(5) \\
& \mathrm{N}(8)-\mathrm{Na}(1)-\mathrm{Na}(2)-\mathrm{Cr}(1) \\
& 84.62(12) \\
& \mathrm{N}(2)-\mathrm{Na}(1)-\mathrm{Na}(2)-\mathrm{Cr}(1) \\
& -41.24(10) \\
& \mathrm{N}(4)-\mathrm{Na}(1)-\mathrm{Na}(2)-\mathrm{Cr}(1) \\
& 157.30(11) \\
& \mathrm{N}(7)-\mathrm{Na}(1)-\mathrm{Na}(2)-\mathrm{Cr}(1) \\
& \text { 43. } 46(12) \\
& \mathrm{C}(3)-\mathrm{Na}(1)-\mathrm{Na}(2)-\mathrm{Cr}(1) \\
& -41.36(12) \\
& \mathrm{C}(39)-\mathrm{Na}(1)-\mathrm{Na}(2)-\mathrm{Cr}(1) \\
& -172.97(12) \\
& \mathrm{C}(54)-\mathrm{Na}(1)-\mathrm{Na}(2)-\mathrm{Cr}(1) \\
& 133.8(5) \\
& \mathrm{C}(4)-\mathrm{Na}(1)-\mathrm{Na}(2)-\mathrm{Cr}(1) \\
& -65.96(11) \\
& \mathrm{Cr}(2)-\mathrm{Na}(1)-\mathrm{Na}(2)-\mathrm{Cr}(1) \\
& 124.87(6) \\
& N(8)-\mathrm{Na}(1)-\mathrm{Na}(2)-\mathrm{C}(40) \\
& -106.20(15) \\
& \mathrm{N}(2)-\mathrm{Na}(1)-\mathrm{Na}(2)-\mathrm{C}(40) \\
& 127.95(15) \\
& \mathrm{N}(4)-\mathrm{Na}(1)-\mathrm{Na}(2)-\mathrm{C}(40) \\
& -33.51(13) \\
& \mathrm{N}(7)-\mathrm{Na}(1)-\mathrm{Na}(2)-\mathrm{C}(40) \\
& -147.35(17) \\
& \mathrm{C}(3)-\mathrm{Na}(1)-\mathrm{Na}(2)-\mathrm{C}(40) \\
& 127.83(16) \\
& \mathrm{C}(39)-\mathrm{Na}(1)-\mathrm{Na}(2)-\mathrm{C}(40) \\
& -3.78(14) \\
& \mathrm{C}(54)-\mathrm{Na}(1)-\mathrm{Na}(2)-\mathrm{C}(40) \\
& -57.0(6) \\
& \mathrm{C}(4)-\mathrm{Na}(1)-\mathrm{Na}(2)-\mathrm{C}(40) \\
& 103.22(16) \\
& \mathrm{Cr}(2)-\mathrm{Na}(1)-\mathrm{Na}(2)-\mathrm{C}(40) \\
& -65.95(10) \\
& \mathrm{Cr}(1)-\mathrm{Na}(1)-\mathrm{Na}(2)-\mathrm{C}(40) \\
& 169.19(12) \\
& \mathrm{N}(8)-\mathrm{Na}(1)-\mathrm{Na}(2)-\mathrm{Cr}(2) \\
& -40.25(11)
\end{aligned}
$$




$$
\begin{aligned}
& \mathrm{N}(2)-\mathrm{Na}(1)-\mathrm{Na}(2)-\mathrm{Cr}(2) \\
& -166.11(12) \\
& \mathrm{N}(4)-\mathrm{Na}(1)-\mathrm{Na}(2)-\mathrm{Cr}(2) \\
& 32.44(9) \\
& \mathrm{N}(7)-\mathrm{Na}(1)-\mathrm{Na}(2)-\mathrm{Cr}(2) \\
& -81.40(13) \\
& \mathrm{C}(3)-\mathrm{Na}(1)-\mathrm{Na}(2)-\mathrm{Cr}(2) \\
& \mathrm{C}(39)-\mathrm{Na}(1)-\mathrm{Na}(2)-\mathrm{Cr}(2) \\
& \mathrm{C}(54)-\mathrm{Na}(1)-\mathrm{Na}(2)-\mathrm{Cr}(2) \\
& \mathrm{C}(4)-\mathrm{Na}(1)-\mathrm{Na}(2)-\mathrm{Cr}(2) \\
& \mathrm{Cr}(1)-\mathrm{Na}(1)-\mathrm{Na}(2)-\mathrm{Cr}(2) \\
& N(7)-C r(1)-N(1)-C(2) \\
& N(2)-C r(1)-N(1)-C(2) \\
& N(3)-C r(1)-N(1)-C(2) \\
& \mathrm{O}(1)-\mathrm{Cr}(1)-\mathrm{N}(1)-\mathrm{C}(2) \\
& \mathrm{Na}(2)-\mathrm{Cr}(1)-\mathrm{N}(1)-\mathrm{C}(2) \\
& \mathrm{Na}(1)-\mathrm{Cr}(1)-\mathrm{N}(1)-\mathrm{C}(2) \\
& N(7)-C r(1)-N(1)-C(10) \\
& N(2)-C r(1)-N(1)-C(10) \\
& N(3)-C r(1)-N(1)-C(10) \\
& O(1)-C r(1)-N(1)-C(10) \\
& \mathrm{Na}(2)-\mathrm{Cr}(1)-\mathrm{N}(1)-\mathrm{C}(10) \\
& \mathrm{Na}(1)-\mathrm{Cr}(1)-\mathrm{N}(1)-\mathrm{C}(10) \\
& N(7)-C r(1)-N(2)-C(3) \\
& N(1)-C r(1)-N(2)-C(3) \\
& N(3)-\operatorname{Cr}(1)-N(2)-C(3) \\
& O(1)-C r(1)-N(2)-C(3) \\
& \mathrm{Na}(2)-\mathrm{Cr}(1)-\mathrm{N}(2)-\mathrm{C}(3) \\
& \mathrm{Na}(1)-\mathrm{Cr}(1)-\mathrm{N}(2)-\mathrm{C}(3) \\
& N(7)-C r(1)-N(2)-C(7) \\
& N(1)-C r(1)-N(2)-C(7) \\
& N(3)-C r(1)-N(2)-C(7) \\
& -166.23(13) \\
& 62.16(10) \\
& 8.9(5) \\
& 169.17(13) \\
& -124.87(6) \\
& 84.3(3) \\
& -13.7(3) \\
& -69.1(4) \\
& -169.8(3) \\
& 37.1(4) \\
& 39.2(3) \\
& -92.0(4) \\
& 170 . \odot(4) \\
& 114.7(4) \\
& \text { 13. } 9(4) \\
& -139.1(3) \\
& -137.1(4) \\
& -95.5(3) \\
& 12.2(3) \\
& 158.2(3) \\
& 87.0(4) \\
& -146.7(3) \\
& -75.3(3) \\
& 89.8(3) \\
& -162.5(3) \\
& -16.5(3)
\end{aligned}
$$




$$
\begin{aligned}
& 0(1)-\operatorname{Cr}(1)-\mathrm{N}(2)-\mathrm{C}(7) \quad-87.6(4) \\
& \mathrm{Na}(2)-\mathrm{Cr}(1)-\mathrm{N}(2)-\mathrm{C}(7) \quad 38.7 \text { (3) } \\
& \mathrm{Na}(1)-\mathrm{Cr}(1)-\mathrm{N}(2)-\mathrm{C}(7) \quad 110.1 \text { (3) } \\
& \mathrm{N}(7)-\mathrm{Cr}(1)-\mathrm{N}(2)-\mathrm{Na}(1) \quad-20.28(16) \\
& \mathrm{N}(1)-\mathrm{Cr}(1)-\mathrm{N}(2)-\mathrm{Na}(1) \quad 87.43(14) \\
& \mathrm{N}(3)-\mathrm{Cr}(1)-\mathrm{N}(2)-\mathrm{Na}(1) \quad-126.57 \text { (15) } \\
& 0(1)-\mathrm{Cr}(1)-\mathrm{N}(2)-\mathrm{Na}(1) \quad 162.3(3) \\
& \mathrm{Na}(2)-\mathrm{Cr}(1)-\mathrm{N}(2)-\mathrm{Na}(1) \quad-71.40(10) \\
& \mathrm{N}(8)-\mathrm{Na}(1)-\mathrm{N}(2)-\mathrm{C}(3) \quad 143.1 \text { (3) } \\
& \mathrm{N}(4)-\mathrm{Na}(1)-\mathrm{N}(2)-\mathrm{C}(3) \quad-145.5(3) \\
& \mathrm{N}(7)-\mathrm{Na}(1)-\mathrm{N}(2)-\mathrm{C}(3) \quad 134.6 \text { (3) } \\
& \mathrm{C}(39)-\mathrm{Na}(1)-\mathrm{N}(2)-\mathrm{C}(3) \quad-123.9(3) \\
& \mathrm{C}(54)-\mathrm{Na}(1)-\mathrm{N}(2)-\mathrm{C}(3) \quad 1.9(3) \\
& \mathrm{C}(4)-\mathrm{Na}(1)-\mathrm{N}(2)-\mathrm{C}(3) \quad-32 . \odot(3) \\
& \mathrm{Cr}(2)-\mathrm{Na}(1)-\mathrm{N}(2)-\mathrm{C}(3) \quad 166.2(2) \\
& \mathrm{Cr}(1)-\mathrm{Na}(1)-\mathrm{N}(2)-\mathrm{C}(3) \quad 120.2(3) \\
& \mathrm{Na}(2)-\mathrm{Na}(1)-\mathrm{N}(2)-\mathrm{C}(3) \quad-179.8 \text { (3) } \\
& \mathrm{N}(8)-\mathrm{Na}(1)-\mathrm{N}(2)-\mathrm{C}(7) \quad-97.3(3) \\
& \mathrm{N}(4)-\mathrm{Na}(1)-\mathrm{N}(2)-\mathrm{C}(7) \quad-25.9(4) \\
& \mathrm{N}(7)-\mathrm{Na}(1)-\mathrm{N}(2)-\mathrm{C}(7) \quad-105.8(3) \\
& \begin{array}{ll}
\mathrm{C}(3)-\mathrm{Na}(1)-\mathrm{N}(2)-\mathrm{C}(7) & 119.7(4)
\end{array} \\
& \mathrm{C}(39)-\mathrm{Na}(1)-\mathrm{N}(2)-\mathrm{C}(7) \quad-4.2(3) \\
& \mathrm{C}(54)-\mathrm{Na}(1)-\mathrm{N}(2)-\mathrm{C}(7) \quad 121.6(3) \\
& \mathrm{C}(4)-\mathrm{Na}(1)-\mathrm{N}(2)-\mathrm{C}(7) \quad 87.7 \text { (3) } \\
& \mathrm{Cr}(2)-\mathrm{Na}(1)-\mathrm{N}(2)-\mathrm{C}(7) \quad-74.1 \text { (3) } \\
& \mathrm{Cr}(1)-\mathrm{Na}(1)-\mathrm{N}(2)-\mathrm{C}(7) \quad-120.2 \text { (3) } \\
& \mathrm{Na}(2)-\mathrm{Na}(1)-\mathrm{N}(2)-\mathrm{C}(7) \quad-60.1(3) \\
& \mathrm{N}(8)-\mathrm{Na}(1)-\mathrm{N}(2)-\mathrm{Cr}(1) \quad 22.90(15)
\end{aligned}
$$




$$
\begin{aligned}
& \mathrm{N}(4)-\mathrm{Na}(1)-\mathrm{N}(2)-\mathrm{Cr}(1) \\
& 94.3(3) \\
& \mathrm{N}(7)-\mathrm{Na}(1)-\mathrm{N}(2)-\mathrm{Cr}(1) \\
& 14.40(12) \\
& \mathrm{C}(3)-\mathrm{Na}(1)-\mathrm{N}(2)-\mathrm{Cr}(1) \\
& -120.2(3) \\
& \mathrm{C}(39)-\mathrm{Na}(1)-\mathrm{N}(2)-\mathrm{Cr}(1) \\
& 115.97(16) \\
& \mathrm{C}(54)-\mathrm{Na}(1)-\mathrm{N}(2)-\mathrm{Cr}(1) \\
& -118 \cdot 3(2) \\
& \mathrm{C}(4)-\mathrm{Na}(1)-\mathrm{N}(2)-\mathrm{Cr}(1) \\
& \mathrm{Cr}(2)-\mathrm{Na}(1)-\mathrm{N}(2)-\mathrm{Cr}(1) \\
& -152.1 \text { (2) } \\
& 46.04(17) \\
& \mathrm{Na}(2)-\mathrm{Na}(1)-\mathrm{N}(2)-\mathrm{Cr}(1) \\
& 60.07(10) \\
& \mathrm{N}(7)-\operatorname{Cr}(1)-\mathrm{N}(3)-\mathrm{C}(8) \\
& \mathrm{N}(2)-\mathrm{Cr}(1)-\mathrm{N}(3)-\mathrm{C}(8) \\
& \mathrm{N}(1)-\mathrm{Cr}(1)-\mathrm{N}(3)-\mathrm{C}(8) \\
& \mathrm{O}(1)-\mathrm{Cr}(1)-\mathrm{N}(3)-\mathrm{C}(8) \\
& \mathrm{Na}(2)-\mathrm{Cr}(1)-\mathrm{N}(3)-\mathrm{C}(8) \\
& \mathrm{Na}(1)-\mathrm{Cr}(1)-\mathrm{N}(3)-\mathrm{C}(8) \\
& \mathrm{N}(7)-\mathrm{Cr}(1)-\mathrm{N}(3)-\mathrm{C}(22) \\
& \mathrm{N}(2)-\mathrm{Cr}(1)-\mathrm{N}(3)-\mathrm{C}(22) \\
& \mathrm{N}(1)-\mathrm{Cr}(1)-\mathrm{N}(3)-\mathrm{C}(22) \\
& O(1)-\operatorname{Cr}(1)-N(3)-C(22) \\
& \mathrm{Na}(2)-\mathrm{Cr}(1)-\mathrm{N}(3)-\mathrm{C}(22) \\
& \mathrm{Na}(1)-\mathrm{Cr}(1)-\mathrm{N}(3)-\mathrm{C}(22) \\
& \mathrm{N}(7)-\mathrm{Cr}(1)-\mathrm{N}(3)-\mathrm{Na}(2) \\
& \mathrm{N}(2)-\mathrm{Cr}(1)-\mathrm{N}(3)-\mathrm{Na}(2) \\
& \mathrm{N}(1)-\mathrm{Cr}(1)-\mathrm{N}(3)-\mathrm{Na}(2) \\
& \mathrm{O}(1)-\mathrm{Cr}(1)-\mathrm{N}(3)-\mathrm{Na}(2) \\
& \mathrm{Na}(1)-\mathrm{Cr}(1)-\mathrm{N}(3)-\mathrm{Na}(2) \\
& N(7)-N a(2)-N(3)-C(8) \\
& N(5)-N a(2)-N(3)-C(8) \\
& \mathrm{C}(44)-\mathrm{Na}(2)-\mathrm{N}(3)-\mathrm{C}(8) \\
& \mathrm{N}(8)-\mathrm{Na}(2)-\mathrm{N}(3)-\mathrm{C}(8) \\
& -82.6(3) \\
& \text { 15. 7(3) } \\
& 71.0(4) \\
& \text { 172.4(3) } \\
& -76 . \odot(3) \\
& -26.3(3) \\
& 126.2(4) \\
& -135.5(4) \\
& -80.2(4) \\
& 21.2(4) \\
& 132.8(4) \\
& -177.5(3) \\
& -6.6 \odot(16) \\
& 91.73(14) \\
& 147.00(18) \\
& -111.60(11) \\
& 49.68(11) \\
& 118.3(3) \\
& 51.6(4) \\
& -34.2(9) \\
& 113.1(2)
\end{aligned}
$$




$$
\begin{aligned}
& \mathrm{C}(43)-\mathrm{Na}(2)-\mathrm{N}(3)-\mathrm{C}(8) \quad-46.4(3) \\
& \mathrm{C}(32)-\mathrm{Na}(2)-\mathrm{N}(3)-\mathrm{C}(8) \quad-114.0(3) \\
& \mathrm{Cr}(1)-\mathrm{Na}(2)-\mathrm{N}(3)-\mathrm{C}(8) \quad 113.7 \text { (3) } \\
& \mathrm{C}(40)-\mathrm{Na}(2)-\mathrm{N}(3)-\mathrm{C}(8) \quad 32 . \odot(3) \\
& \mathrm{Cr}(2)-\mathrm{Na}(2)-\mathrm{N}(3)-\mathrm{C}(8) \quad 101.3(3) \\
& \mathrm{Na}(1)-\mathrm{Na}(2)-\mathrm{N}(3)-\mathrm{C}(8) \quad 74.7 \text { (2) } \\
& \mathrm{N}(7)-\mathrm{Na}(2)-\mathrm{N}(3)-\mathrm{C}(22) \quad-128.6(3) \\
& \mathrm{N}(5)-\mathrm{Na}(2)-\mathrm{N}(3)-\mathrm{C}(22) \quad 164.7 \text { (3) } \\
& \mathrm{C}(44)-\mathrm{Na}(2)-\mathrm{N}(3)-\mathrm{C}(22) \quad 78.9(9) \\
& \mathrm{C}(8)-\mathrm{Na}(2)-\mathrm{N}(3)-\mathrm{C}(22) \quad 113.1(4) \\
& \mathrm{N}(8)-\mathrm{Na}(2)-\mathrm{N}(3)-\mathrm{C}(22) \quad-133.8(3) \\
& \mathrm{C}(43)-\mathrm{Na}(2)-\mathrm{N}(3)-\mathrm{C}(22) \quad 66.7 \text { (4) } \\
& \mathrm{C}(32)-\mathrm{Na}(2)-\mathrm{N}(3)-\mathrm{C}(22) \quad-0.9(3) \\
& \mathrm{Cr}(1)-\mathrm{Na}(2)-\mathrm{N}(3)-\mathrm{C}(22) \quad-133.2(4) \\
& \mathrm{C}(40)-\mathrm{Na}(2)-\mathrm{N}(3)-\mathrm{C}(22) \quad 145.1 \text { (3) } \\
& \operatorname{Cr}(2)-\mathrm{Na}(2)-\mathrm{N}(3)-\mathrm{C}(22) \quad-145.6(3) \\
& \mathrm{Na}(1)-\mathrm{Na}(2)-\mathrm{N}(3)-\mathrm{C}(22) \quad-172.2 \text { (3) } \\
& \mathrm{N}(7)-\mathrm{Na}(2)-\mathrm{N}(3)-\mathrm{Cr}(1) \quad 4.58 \text { (11) } \\
& \mathrm{N}(5)-\mathrm{Na}(2)-\mathrm{N}(3)-\mathrm{Cr}(1) \quad-62.1 \text { (3) } \\
& \mathrm{C}(44)-\mathrm{Na}(2)-\mathrm{N}(3)-\mathrm{Cr}(1) \quad-147.9(8) \\
& \mathrm{C}(8)-\mathrm{Na}(2)-\mathrm{N}(3)-\mathrm{Cr}(1) \quad-113.7 \text { (3) } \\
& \mathrm{N}(8)-\mathrm{Na}(2)-\mathrm{N}(3)-\mathrm{Cr}(1) \quad-0.59(14) \\
& \mathrm{C}(43)-\mathrm{Na}(2)-\mathrm{N}(3)-\mathrm{Cr}(1) \quad-160.1 \text { (2) } \\
& \mathrm{C}(32)-\mathrm{Na}(2)-\mathrm{N}(3)-\mathrm{Cr}(1) \quad 132.32(18) \\
& \mathrm{C}(40)-\mathrm{Na}(2)-\mathrm{N}(3)-\mathrm{Cr}(1) \quad-81.71(17) \\
& \mathrm{Cr}(2)-\mathrm{Na}(2)-\mathrm{N}(3)-\mathrm{Cr}(1) \quad-12.34(19) \\
& \mathrm{Na}(1)-\mathrm{Na}(2)-\mathrm{N}(3)-\mathrm{Cr}(1) \quad-39.01(10) \\
& \mathrm{N}(8)-\mathrm{Cr}(2)-\mathrm{N}(4)-\mathrm{C}(39) \quad-78.7(3)
\end{aligned}
$$




$$
\begin{aligned}
& \mathrm{N}(5)-\mathrm{Cr}(2)-\mathrm{N}(4)-\mathrm{C}(39) \quad 17.5(3) \\
& \mathrm{N}(6)-\mathrm{Cr}(2)-\mathrm{N}(4)-\mathrm{C}(39) \quad 71.6(3) \\
& \mathrm{O}(2)-\mathrm{Cr}(2)-\mathrm{N}(4)-\mathrm{C}(39) \quad 174.4(3) \\
& \mathrm{Na}(1)-\mathrm{Cr}(2)-\mathrm{N}(4)-\mathrm{C}(39) \quad-74.9 \text { (3) } \\
& \mathrm{Na}(2)-\mathrm{Cr}(2)-\mathrm{N}(4)-\mathrm{C}(39) \quad-23.3(3) \\
& \mathrm{N}(8)-\mathrm{Cr}(2)-\mathrm{N}(4)-\mathrm{C}(47) \quad 126.8(4) \\
& \mathrm{N}(5)-\mathrm{Cr}(2)-\mathrm{N}(4)-\mathrm{C}(47) \quad-137.0(4) \\
& \mathrm{N}(6)-\mathrm{Cr}(2)-\mathrm{N}(4)-\mathrm{C}(47) \quad-82.9(4) \\
& \mathrm{O}(2)-\mathrm{Cr}(2)-\mathrm{N}(4)-\mathrm{C}(47) \quad 19.9(4) \\
& \mathrm{Na}(1)-\mathrm{Cr}(2)-\mathrm{N}(4)-\mathrm{C}(47) \quad 130.6(4) \\
& \mathrm{Na}(2)-\mathrm{Cr}(2)-\mathrm{N}(4)-\mathrm{C}(47) \quad-177.8(3) \\
& \mathrm{N}(8)-\mathrm{Cr}(2)-\mathrm{N}(4)-\mathrm{Na}(1) \quad-3.85(16) \\
& \mathrm{N}(5)-\mathrm{Cr}(2)-\mathrm{N}(4)-\mathrm{Na}(1) \quad 92.36(13) \\
& \mathrm{N}(6)-\mathrm{Cr}(2)-\mathrm{N}(4)-\mathrm{Na}(1) \quad 146.43(17) \\
& \mathrm{O}(2)-\mathrm{Cr}(2)-\mathrm{N}(4)-\mathrm{Na}(1) \quad-110.71 \text { (11) } \\
& \mathrm{Na}(2)-\mathrm{Cr}(2)-\mathrm{N}(4)-\mathrm{Na}(1) \quad 51.54(10) \\
& \mathrm{N}(8)-\mathrm{Na}(1)-\mathrm{N}(4)-\mathrm{C}(39) \quad 116.6(2) \\
& \mathrm{N}(2)-\mathrm{Na}(1)-\mathrm{N}(4)-\mathrm{C}(39) \quad 38.9(3) \\
& \mathrm{N}(7)-\mathrm{Na}(1)-\mathrm{N}(4)-\mathrm{C}(39) \quad 107.9(2) \\
& \mathrm{C}(3)-\mathrm{Na}(1)-\mathrm{N}(4)-\mathrm{C}(39) \quad-26.4(6) \\
& \mathrm{C}(54)-\mathrm{Na}(1)-\mathrm{N}(4)-\mathrm{C}(39) \quad-116.6 \text { (3) } \\
& \mathrm{C}(4)-\mathrm{Na}(1)-\mathrm{N}(4)-\mathrm{C}(39) \quad-4 \odot .9 \text { (3) } \\
& \begin{array}{ll}
\mathrm{Cr}(2)-\mathrm{Na}(1)-\mathrm{N}(4)-\mathrm{C}(39) & 113.9(3)
\end{array} \\
& \operatorname{Cr}(1)-\mathrm{Na}(1)-\mathrm{N}(4)-\mathrm{C}(39) \quad 94.1 \text { (3) } \\
& \mathrm{Na}(2)-\mathrm{Na}(1)-\mathrm{N}(4)-\mathrm{C}(39) \quad 69.4 \text { (2) } \\
& \mathrm{N}(8)-\mathrm{Na}(1)-\mathrm{N}(4)-\mathrm{C}(47) \quad-132 . \odot(3) \\
& \mathrm{N}(2)-\mathrm{Na}(1)-\mathrm{N}(4)-\mathrm{C}(47) \quad 150.3(3) \\
& \mathrm{N}(7)-\mathrm{Na}(1)-\mathrm{N}(4)-\mathrm{C}(47) \quad-140.6 \text { (3) } \\
& \begin{array}{ll}
\mathrm{C}(3)-\mathrm{Na}(1)-\mathrm{N}(4)-\mathrm{C}(47) \quad 85.0(6)
\end{array}
\end{aligned}
$$




$$
\begin{aligned}
& \begin{array}{ll}
\mathrm{C}(39)-\mathrm{Na}(1)-\mathrm{N}(4)-\mathrm{C}(47) & 111.4(4)
\end{array} \\
& \mathrm{C}(54)-\mathrm{Na}(1)-\mathrm{N}(4)-\mathrm{C}(47) \quad-5.1(3) \\
& \mathrm{C}(4)-\mathrm{Na}(1)-\mathrm{N}(4)-\mathrm{C}(47) \quad 70.5(4) \\
& \mathrm{Cr}(2)-\mathrm{Na}(1)-\mathrm{N}(4)-\mathrm{C}(47) \quad-134.7(4) \\
& \mathrm{Cr}(1)-\mathrm{Na}(1)-\mathrm{N}(4)-\mathrm{C}(47) \quad-154.5(3) \\
& \mathrm{Na}(2)-\mathrm{Na}(1)-\mathrm{N}(4)-\mathrm{C}(47) \quad-179.2(3) \\
& \mathrm{N}(8)-\mathrm{Na}(1)-\mathrm{N}(4)-\mathrm{Cr}(2) \quad 2.70(11) \\
& \mathrm{N}(2)-\mathrm{Na}(1)-\mathrm{N}(4)-\mathrm{Cr}(2) \quad-75 . \odot(3) \\
& \mathrm{N}(7)-\mathrm{Na}(1)-\mathrm{N}(4)-\mathrm{Cr}(2) \quad-5.92(14) \\
& \mathrm{C}(3)-\mathrm{Na}(1)-\mathrm{N}(4)-\mathrm{Cr}(2) \quad-140.3(4) \\
& \mathrm{C}(39)-\mathrm{Na}(1)-\mathrm{N}(4)-\mathrm{Cr}(2) \quad-113.9(3) \\
& \mathrm{C}(54)-\mathrm{Na}(1)-\mathrm{N}(4)-\mathrm{Cr}(2) \quad 129.59(16) \\
& \mathrm{C}(4)-\mathrm{Na}(1)-\mathrm{N}(4)-\mathrm{Cr}(2) \quad-154.77(18) \\
& \mathrm{Cr}(1)-\mathrm{Na}(1)-\mathrm{N}(4)-\mathrm{Cr}(2) \quad-19.80(19) \\
& \mathrm{Na}(2)-\mathrm{Na}(1)-\mathrm{N}(4)-\mathrm{Cr}(2) \quad-44.48(10) \\
& N(8)-C r(2)-N(5)-C(44) \quad-93.6(3) \\
& N(6)-C r(2)-N(5)-C(44) \quad 14.2(3) \\
& N(4)-\operatorname{Cr}(2)-N(5)-C(44) \quad 161.2(3) \\
& O(2)-\operatorname{Cr}(2)-N(5)-C(44) \quad 90.2(4) \\
& \mathrm{Na}(1)-\mathrm{Cr}(2)-\mathrm{N}(5)-\mathrm{C}(44) \quad-143.7(3) \\
& \mathrm{Na}(2)-\mathrm{Cr}(2)-\mathrm{N}(5)-\mathrm{C}(44) \quad-77.5(3) \\
& N(8)-C r(2)-N(5)-C(40) \quad 86.4(3) \\
& N(6)-\operatorname{Cr}(2)-N(5)-C(40) \quad-165.8(3) \\
& N(4)-\operatorname{Cr}(2)-N(5)-C(40) \quad-18.9(3) \\
& O(2)-\operatorname{Cr}(2)-N(5)-C(40) \quad-89.9(4) \\
& \mathrm{Na}(1)-\mathrm{Cr}(2)-\mathrm{N}(5)-\mathrm{C}(40) \quad 36.3(3) \\
& \mathrm{Na}(2)-\mathrm{Cr}(2)-\mathrm{N}(5)-\mathrm{C}(40) \quad 102.5(3) \\
& \mathrm{N}(8)-\mathrm{Cr}(2)-\mathrm{N}(5)-\mathrm{Na}(2) \quad-16.07(16)
\end{aligned}
$$




$$
\begin{aligned}
& \mathrm{N}(6)-\mathrm{Cr}(2)-\mathrm{N}(5)-\mathrm{Na}(2) \quad 91.70(15) \\
& \mathrm{N}(4)-\mathrm{Cr}(2)-\mathrm{N}(5)-\mathrm{Na}(2) \quad-121.32(15) \\
& \mathrm{O}(2)-\mathrm{Cr}(2)-\mathrm{N}(5)-\mathrm{Na}(2) \quad 167.7 \text { (3) } \\
& \mathrm{Na}(1)-\mathrm{Cr}(2)-\mathrm{N}(5)-\mathrm{Na}(2) \quad-66.20(11) \\
& \mathrm{N}(7)-\mathrm{Na}(2)-\mathrm{N}(5)-\mathrm{C}(44) \quad 138.6(3) \\
& \mathrm{N}(3)-\mathrm{Na}(2)-\mathrm{N}(5)-\mathrm{C}(44) \quad-158.0(3) \\
& \mathrm{C}(8)-\mathrm{Na}(2)-\mathrm{N}(5)-\mathrm{C}(44) \quad-127.9(3) \\
& \begin{array}{ll}
\mathrm{N}(8)-\mathrm{Na}(2)-\mathrm{N}(5)-\mathrm{C}(44) & 130.1(3)
\end{array} \\
& \mathrm{C}(43)-\mathrm{Na}(2)-\mathrm{N}(5)-\mathrm{C}(44) \quad-32.0(2) \\
& \mathrm{C}(32)-\mathrm{Na}(2)-\mathrm{N}(5)-\mathrm{C}(44) \quad 3.4(3) \\
& \mathrm{Cr}(1)-\mathrm{Na}(2)-\mathrm{N}(5)-\mathrm{C}(44) \quad 159.3(2) \\
& \mathrm{C}(40)-\mathrm{Na}(2)-\mathrm{N}(5)-\mathrm{C}(44) \quad-121.3(4) \\
& \begin{array}{ll}
\mathrm{Cr}(2)-\mathrm{Na}(2)-\mathrm{N}(5)-\mathrm{C}(44) \quad 118.9(3)
\end{array} \\
& \mathrm{Na}(1)-\mathrm{Na}(2)-\mathrm{N}(5)-\mathrm{C}(44) \quad 176.5(3) \\
& \mathrm{N}(7)-\mathrm{Na}(2)-\mathrm{N}(5)-\mathrm{C}(40) \quad-100.2(3) \\
& \mathrm{N}(3)-\mathrm{Na}(2)-\mathrm{N}(5)-\mathrm{C}(40) \quad-36.8(4) \\
& \mathrm{C}(44)-\mathrm{Na}(2)-\mathrm{N}(5)-\mathrm{C}(40) \quad 121.3(4) \\
& \begin{array}{ll}
\mathrm{C}(8)-\mathrm{Na}(2)-\mathrm{N}(5)-\mathrm{C}(40) & -6.7(3)
\end{array} \\
& \mathrm{N}(8)-\mathrm{Na}(2)-\mathrm{N}(5)-\mathrm{C}(40) \quad-108.6 \text { (3) } \\
& \mathrm{C}(43)-\mathrm{Na}(2)-\mathrm{N}(5)-\mathrm{C}(40) \quad 89.3(3) \\
& \mathrm{C}(32)-\mathrm{Na}(2)-\mathrm{N}(5)-\mathrm{C}(4 \odot) \quad 124.7 \text { (3) } \\
& \mathrm{Cr}(1)-\mathrm{Na}(2)-\mathrm{N}(5)-\mathrm{C}(40) \quad-79.5(3) \\
& \mathrm{Cr}(2)-\mathrm{Na}(2)-\mathrm{N}(5)-\mathrm{C}(40) \quad-119.8 \text { (3) } \\
& \mathrm{Na}(1)-\mathrm{Na}(2)-\mathrm{N}(5)-\mathrm{C}(40) \quad-62.2(2) \\
& \mathrm{N}(7)-\mathrm{Na}(2)-\mathrm{N}(5)-\mathrm{Cr}(2) \quad 19.65 \text { (15) } \\
& \mathrm{N}(3)-\mathrm{Na}(2)-\mathrm{N}(5)-\mathrm{Cr}(2) \quad 83.0(3) \\
& \mathrm{C}(44)-\mathrm{Na}(2)-\mathrm{N}(5)-\mathrm{Cr}(2) \quad-118.9 \text { (3) } \\
& \mathrm{C}(8)-\mathrm{Na}(2)-\mathrm{N}(5)-\mathrm{Cr}(2) \quad 113.13(18) \\
& \mathrm{N}(8)-\mathrm{Na}(2)-\mathrm{N}(5)-\mathrm{Cr}(2) \quad 11.16(12)
\end{aligned}
$$




$$
\begin{aligned}
& \mathrm{C}(43)-\mathrm{Na}(2)-\mathrm{N}(5)-\mathrm{Cr}(2) \quad-150.9(2) \\
& \mathrm{C}(32)-\mathrm{Na}(2)-\mathrm{N}(5)-\mathrm{Cr}(2) \quad-115.5(2) \\
& \mathrm{Cr}(1)-\mathrm{Na}(2)-\mathrm{N}(5)-\mathrm{Cr}(2) \quad 40.34(17) \\
& \mathrm{C}(40)-\mathrm{Na}(2)-\mathrm{N}(5)-\mathrm{Cr}(2) \quad 119.8 \text { (3) } \\
& \mathrm{Na}(1)-\mathrm{Na}(2)-\mathrm{N}(5)-\mathrm{Cr}(2) \quad 57.56 \text { (11) } \\
& \mathrm{N}(8)-\mathrm{Cr}(2)-\mathrm{N}(6)-\mathrm{C}(45) \quad 80.4(3) \\
& N(5)-C r(2)-N(6)-C(45) \quad-15.2(3) \\
& \mathrm{N}(4)-\mathrm{Cr}(2)-\mathrm{N}(6)-\mathrm{C}(45) \quad-69.4(4) \\
& \mathrm{O}(2)-\mathrm{Cr}(2)-\mathrm{N}(6)-\mathrm{C}(45) \quad-171.4(3) \\
& \mathrm{Na}(1)-\mathrm{Cr}(2)-\mathrm{N}(6)-\mathrm{C}(45) \quad 37.3(4) \\
& \mathrm{Na}(2)-\mathrm{Cr}(2)-\mathrm{N}(6)-\mathrm{C}(45) \quad 33.3(3) \\
& \mathrm{N}(8)-\mathrm{Cr}(2)-\mathrm{N}(6)-\mathrm{C}(59) \quad-90.2(4) \\
& \mathrm{N}(5)-\mathrm{Cr}(2)-\mathrm{N}(6)-\mathrm{C}(59) \quad 174.2(4) \\
& \mathrm{N}(4)-\mathrm{Cr}(2)-\mathrm{N}(6)-\mathrm{C}(59) \quad 120 . \Theta(4) \\
& 0(2)-\mathrm{Cr}(2)-\mathrm{N}(6)-\mathrm{C}(59) \quad 18.0(4) \\
& \mathrm{Na}(1)-\mathrm{Cr}(2)-\mathrm{N}(6)-\mathrm{C}(59) \quad-133.3(3) \\
& \mathrm{Na}(2)-\mathrm{Cr}(2)-\mathrm{N}(6)-\mathrm{C}(59) \quad-137.3(4) \\
& N(2)-\operatorname{Cr}(1)-N(7)-N(8) \quad 14.7 \text { (11) } \\
& \mathrm{N}(1)-\mathrm{Cr}(1)-\mathrm{N}(7)-\mathrm{N}(8) \quad-66.1 \text { (11) } \\
& N(3)-\operatorname{Cr}(1)-N(7)-N(8) \quad 95.6(11) \\
& O(1)-\operatorname{Cr}(1)-N(7)-N(8) \quad-166.5(11) \\
& \mathrm{Na}(2)-\mathrm{Cr}(1)-\mathrm{N}(7)-\mathrm{N}(8) \quad 88.9 \text { (11) } \\
& \mathrm{Na}(1)-\mathrm{Cr}(1)-\mathrm{N}(7)-\mathrm{N}(8) \quad-5.3(10) \\
& \mathrm{N}(2)-\mathrm{Cr}(1)-\mathrm{N}(7)-\mathrm{Na}(2) \quad-74.21(15) \\
& \mathrm{N}(1)-\mathrm{Cr}(1)-\mathrm{N}(7)-\mathrm{Na}(2) \quad-155.02 \text { (13) } \\
& \mathrm{N}(3)-\mathrm{Cr}(1)-\mathrm{N}(7)-\mathrm{Na}(2) \quad 6.71(17) \\
& \mathrm{O}(1)-\mathrm{Cr}(1)-\mathrm{N}(7)-\mathrm{Na}(2) \quad 104.67 \text { (13) } \\
& \mathrm{Na}(1)-\mathrm{Cr}(1)-\mathrm{N}(7)-\mathrm{Na}(2) \quad-94.18(14)
\end{aligned}
$$




$$
\begin{aligned}
& N(2)-\mathrm{Cr}(1)-\mathrm{N}(7)-\mathrm{Na}(1) \\
& 19.96(16) \\
& \mathrm{N}(1)-\mathrm{Cr}(1)-\mathrm{N}(7)-\mathrm{Na}(1) \\
& -60.84(16) \\
& \mathrm{N}(3)-\mathrm{Cr}(1)-\mathrm{N}(7)-\mathrm{Na}(1) \\
& 100.88(14) \\
& \mathrm{O}(1)-\mathrm{Cr}(1)-\mathrm{N}(7)-\mathrm{Na}(1) \\
& -161.15(12) \\
& \mathrm{Na}(2)-\mathrm{Cr}(1)-\mathrm{N}(7)-\mathrm{Na}(1) \\
& 94.18(14) \\
& \mathrm{N}(3)-\mathrm{Na}(2)-\mathrm{N}(7)-\mathrm{N}(8) \\
& \mathrm{N}(5)-\mathrm{Na}(2)-\mathrm{N}(7)-\mathrm{N}(8) \\
& \mathrm{C}(44)-\mathrm{Na}(2)-\mathrm{N}(7)-\mathrm{N}(8) \\
& \mathrm{C}(8)-\mathrm{Na}(2)-\mathrm{N}(7)-\mathrm{N}(8) \\
& \mathrm{C}(43)-\mathrm{Na}(2)-\mathrm{N}(7)-\mathrm{N}(8) \\
& \mathrm{C}(32)-\mathrm{Na}(2)-\mathrm{N}(7)-\mathrm{N}(8) \\
& \mathrm{Cr}(1)-\mathrm{Na}(2)-\mathrm{N}(7)-\mathrm{N}(8) \\
& C(40)-\mathrm{Na}(2)-\mathrm{N}(7)-\mathrm{N}(8) \\
& \mathrm{Cr}(2)-\mathrm{Na}(2)-\mathrm{N}(7)-\mathrm{N}(8) \\
& \mathrm{Na}(1)-\mathrm{Na}(2)-\mathrm{N}(7)-\mathrm{N}(8) \\
& \mathrm{N}(3)-\mathrm{Na}(2)-\mathrm{N}(7)-\mathrm{Cr}(1) \\
& \mathrm{N}(5)-\mathrm{Na}(2)-\mathrm{N}(7)-\mathrm{Cr}(1) \\
& \mathrm{C}(44)-\mathrm{Na}(2)-\mathrm{N}(7)-\mathrm{Cr}(1) \\
& \mathrm{C}(8)-\mathrm{Na}(2)-\mathrm{N}(7)-\mathrm{Cr}(1) \\
& \mathrm{N}(8)-\mathrm{Na}(2)-\mathrm{N}(7)-\mathrm{Cr}(1) \\
& \mathrm{C}(43)-\mathrm{Na}(2)-\mathrm{N}(7)-\mathrm{Cr}(1) \\
& \mathrm{C}(32)-\mathrm{Na}(2)-\mathrm{N}(7)-\mathrm{Cr}(1) \\
& \mathrm{C}(40)-\mathrm{Na}(2)-\mathrm{N}(7)-\mathrm{Cr}(1) \\
& \mathrm{Cr}(2)-\mathrm{Na}(2)-\mathrm{N}(7)-\mathrm{Cr}(1) \\
& \mathrm{Na}(1)-\mathrm{Na}(2)-\mathrm{N}(7)-\mathrm{Cr}(1) \\
& \mathrm{N}(3)-\mathrm{Na}(2)-\mathrm{N}(7)-\mathrm{Na}(1) \\
& N(5)-N a(2)-N(7)-N a(1) \\
& \mathrm{C}(44)-\mathrm{Na}(2)-\mathrm{N}(7)-\mathrm{Na}(1) \\
& \mathrm{C}(8)-\mathrm{Na}(2)-\mathrm{N}(7)-\mathrm{Na}(1) \\
& -169.2(2) \\
& -16.4(2) \\
& 5.2(3) \\
& -141.8(2) \\
& -4.7(3) \\
& 123.8(3) \\
& -163.8(3) \\
& -43 . \odot(2) \\
& -3.65(18) \\
& -69.6(2) \\
& -5.36(13) \\
& 147.40(15) \\
& 169.09(15) \\
& 22.07(16) \\
& 163.8(3) \\
& 159.1(2) \\
& -72.4(2) \\
& 120.86(14) \\
& 160.19(16) \\
& 94.26 \text { (15) } \\
& -99.63(13) \\
& 53.14(13) \\
& 74.82(17) \\
& -72.19(15)
\end{aligned}
$$




$$
\begin{aligned}
& \mathrm{N}(8)-\mathrm{Na}(2)-\mathrm{N}(7)-\mathrm{Na}(1) \quad 69.6 \text { (2) } \\
& \mathrm{C}(43)-\mathrm{Na}(2)-\mathrm{N}(7)-\mathrm{Na}(1) \quad 64.9 \text { (3) } \\
& \mathrm{C}(32)-\mathrm{Na}(2)-\mathrm{N}(7)-\mathrm{Na}(1) \quad-166.66 \text { (17) } \\
& \operatorname{Cr}(1)-\mathrm{Na}(2)-\mathrm{N}(7)-\mathrm{Na}(1) \quad-94.26(15) \\
& \mathrm{C}(40)-\mathrm{Na}(2)-\mathrm{N}(7)-\mathrm{Na}(1) \quad 26.60(13) \\
& \mathrm{Cr}(2)-\mathrm{Na}(2)-\mathrm{N}(7)-\mathrm{Na}(1) \quad 65.93(9) \\
& N(2)-\mathrm{Na}(1)-\mathrm{N}(7)-\mathrm{N}(8) \quad 162.5(3) \\
& \mathrm{N}(4)-\mathrm{Na}(1)-\mathrm{N}(7)-\mathrm{N}(8) \quad 17.2(2) \\
& \mathrm{C}(3)-\mathrm{Na}(1)-\mathrm{N}(7)-\mathrm{N}(8) \quad-176 . \odot(2) \\
& \mathrm{C}(39)-\mathrm{Na}(1)-\mathrm{N}(7)-\mathrm{N}(8) \quad 48.7(3) \\
& \mathrm{C}(54)-\mathrm{Na}(1)-\mathrm{N}(7)-\mathrm{N}(8) \quad-71.6(3) \\
& \mathrm{C}(4)-\mathrm{Na}(1)-\mathrm{N}(7)-\mathrm{N}(8) \quad 173.6(2) \\
& \operatorname{Cr}(2)-\mathrm{Na}(1)-\mathrm{N}(7)-\mathrm{N}(8) \quad 12.81 \text { (19) } \\
& \operatorname{Cr}(1)-\mathrm{Na}(1)-\mathrm{N}(7)-\mathrm{N}(8) \quad 178.4 \text { (3) } \\
& \mathrm{Na}(2)-\mathrm{Na}(1)-\mathrm{N}(7)-\mathrm{N}(8) \quad 86.7 \text { (2) } \\
& \mathrm{N}(8)-\mathrm{Na}(1)-\mathrm{N}(7)-\mathrm{Cr}(1) \quad-178.4 \text { (3) } \\
& \mathrm{N}(2)-\mathrm{Na}(1)-\mathrm{N}(7)-\mathrm{Cr}(1) \quad-15.95 \text { (13) } \\
& \mathrm{N}(4)-\mathrm{Na}(1)-\mathrm{N}(7)-\mathrm{Cr}(1) \quad-161.22(14) \\
& \mathrm{C}(3)-\mathrm{Na}(1)-\mathrm{N}(7)-\mathrm{Cr}(1) \quad 5.54 \text { (17) } \\
& \mathrm{C}(39)-\mathrm{Na}(1)-\mathrm{N}(7)-\mathrm{Cr}(1) \quad-129.71(16) \\
& \begin{array}{ll}
\mathrm{C}(54)-\mathrm{Na}(1)-\mathrm{N}(7)-\mathrm{Cr}(1) & 110.0(2)
\end{array} \\
& \mathrm{C}(4)-\mathrm{Na}(1)-\mathrm{N}(7)-\mathrm{Cr}(1) \quad-4.8(2) \\
& \operatorname{Cr}(2)-\mathrm{Na}(1)-\mathrm{N}(7)-\mathrm{Cr}(1) \quad-165.62(16) \\
& \mathrm{Na}(2)-\mathrm{Na}(1)-\mathrm{N}(7)-\mathrm{Cr}(1) \quad-91.71(16) \\
& \mathrm{N}(8)-\mathrm{Na}(1)-\mathrm{N}(7)-\mathrm{Na}(2) \quad-86.7 \text { (2) } \\
& \mathrm{N}(2)-\mathrm{Na}(1)-\mathrm{N}(7)-\mathrm{Na}(2) \quad 75.76 \text { (13) } \\
& \mathrm{N}(4)-\mathrm{Na}(1)-\mathrm{N}(7)-\mathrm{Na}(2) \quad-69.50(15) \\
& \mathrm{C}(3)-\mathrm{Na}(1)-\mathrm{N}(7)-\mathrm{Na}(2) \quad 97.25 \text { (15) }
\end{aligned}
$$




$$
\begin{aligned}
& \mathrm{C}(39)-\mathrm{Na}(1)-\mathrm{N}(7)-\mathrm{Na}(2) \\
& -38.00(17) \\
& \mathrm{C}(54)-\mathrm{Na}(1)-\mathrm{N}(7)-\mathrm{Na}(2) \\
& -158.30(17) \\
& \mathrm{C}(4)-\mathrm{Na}(1)-\mathrm{N}(7)-\mathrm{Na}(2) \\
& \text { 86. 91(17) } \\
& \mathrm{Cr}(2)-\mathrm{Na}(1)-\mathrm{N}(7)-\mathrm{Na}(2) \\
& -73.90(10) \\
& \mathrm{Cr}(1)-\mathrm{Na}(1)-\mathrm{N}(7)-\mathrm{Na}(2) \\
& 91.71(16) \\
& \operatorname{Cr}(1)-\mathrm{N}(7)-\mathrm{N}(8)-\mathrm{Cr}(2) \\
& -72.1(15) \\
& \mathrm{Na}(2)-\mathrm{N}(7)-\mathrm{N}(8)-\mathrm{Cr}(2) \\
& 17.2(8) \\
& \mathrm{Na}(1)-\mathrm{N}(7)-\mathrm{N}(8)-\mathrm{Cr}(2) \\
& -77.8(8) \\
& \mathrm{Cr}(1)-\mathrm{N}(7)-\mathrm{N}(8)-\mathrm{Na}(1) \\
& 5.6(11) \\
& \mathrm{Na}(2)-\mathrm{N}(7)-\mathrm{N}(8)-\mathrm{Na}(1) \\
& 94.95(12) \\
& \mathrm{Cr}(1)-\mathrm{N}(7)-\mathrm{N}(8)-\mathrm{Na}(2) \\
& -89.3(11) \\
& \mathrm{Na}(1)-\mathrm{N}(7)-\mathrm{N}(8)-\mathrm{Na}(2) \\
& -94.95(12) \\
& N(5)-\operatorname{Cr}(2)-N(8)-N(7) \\
& -0.6(9) \\
& \mathrm{N}(6)-\operatorname{Cr}(2)-\mathrm{N}(8)-\mathrm{N}(7) \\
& \mathrm{N}(4)-\mathrm{Cr}(2)-\mathrm{N}(8)-\mathrm{N}(7) \\
& O(2)-\operatorname{Cr}(2)-N(8)-N(7) \\
& \mathrm{Na}(1)-\mathrm{Cr}(2)-\mathrm{N}(8)-\mathrm{N}(7) \\
& \mathrm{Na}(2)-\mathrm{Cr}(2)-\mathrm{N}(8)-\mathrm{N}(7) \\
& \mathrm{N}(5)-\mathrm{Cr}(2)-\mathrm{N}(8)-\mathrm{Na}(1) \\
& \mathrm{N}(6)-\mathrm{Cr}(2)-\mathrm{N}(8)-\mathrm{Na}(1) \\
& \mathrm{N}(4)-\mathrm{Cr}(2)-\mathrm{N}(8)-\mathrm{Na}(1) \\
& \mathrm{O}(2)-\mathrm{Cr}(2)-\mathrm{N}(8)-\mathrm{Na}(1) \\
& \mathrm{Na}(2)-\mathrm{Cr}(2)-\mathrm{N}(8)-\mathrm{Na}(1) \\
& \mathrm{N}(5)-\mathrm{Cr}(2)-\mathrm{N}(8)-\mathrm{Na}(2) \\
& \mathrm{N}(6)-\mathrm{Cr}(2)-\mathrm{N}(8)-\mathrm{Na}(2) \\
& \mathrm{N}(4)-\mathrm{Cr}(2)-\mathrm{N}(8)-\mathrm{Na}(2) \\
& \mathrm{O}(2)-\mathrm{Cr}(2)-\mathrm{N}(8)-\mathrm{Na}(2) \\
& \mathrm{Na}(1)-\mathrm{Cr}(2)-\mathrm{N}(8)-\mathrm{Na}(2) \\
& -80.5(8) \\
& 79.4(8) \\
& 177.8(8) \\
& 75.4(8) \\
& -15.5(8) \\
& -75.98(15) \\
& -155.83(13) \\
& 3.99(17) \\
& 102.42(13) \\
& -90.84(14) \\
& 14.86(15) \\
& -64.99(16) \\
& 94.83(14) \\
& -166.74(11) \\
& 90.84(14) \\
& N(2)-\mathrm{Na}(1)-\mathrm{N}(8)-\mathrm{N}(7) \\
& -16.2(2)
\end{aligned}
$$




$$
\begin{aligned}
& N(4)-\mathrm{Na}(1)-\mathrm{N}(8)-\mathrm{N}(7) \\
& -162.6(2) \\
& \mathrm{C}(3)-\mathrm{Na}(1)-\mathrm{N}(8)-\mathrm{N}(7) \\
& 4.6(3) \\
& \mathrm{C}(39)-\mathrm{Na}(1)-\mathrm{N}(8)-\mathrm{N}(7) \\
& -135.2(2) \\
& \mathrm{C}(54)-\mathrm{Na}(1)-\mathrm{N}(8)-\mathrm{N}(7) \\
& 129.7(2) \\
& \mathrm{C}(4)-\mathrm{Na}(1)-\mathrm{N}(8)-\mathrm{N}(7) \\
& -9.9(4) \\
& \mathrm{Cr}(2)-\mathrm{Na}(1)-\mathrm{N}(8)-\mathrm{N}(7) \\
& -159.4(3) \\
& \mathrm{Cr}(1)-\mathrm{Na}(1)-\mathrm{N}(8)-\mathrm{N}(7) \\
& -0.96(18) \\
& \mathrm{Na}(2)-\mathrm{Na}(1)-\mathrm{N}(8)-\mathrm{N}(7) \\
& -63.4(2) \\
& N(2)-N a(1)-N(8)-C r(2) \\
& 143.19(15) \\
& \mathrm{N}(4)-\mathrm{Na}(1)-\mathrm{N}(8)-\mathrm{Cr}(2) \\
& -3.18(13) \\
& \mathrm{N}(7)-\mathrm{Na}(1)-\mathrm{N}(8)-\mathrm{Cr}(2) \\
& \mathrm{C}(3)-\mathrm{Na}(1)-\mathrm{N}(8)-\mathrm{Cr}(2) \\
& 159.4(3) \\
& 164.00(16) \\
& \mathrm{C}(39)-\mathrm{Na}(1)-\mathrm{N}(8)-\mathrm{Cr}(2) \\
& 24.27(16) \\
& \mathrm{C}(54)-\mathrm{Na}(1)-\mathrm{N}(8)-\mathrm{Cr}(2) \\
& -70.8(2) \\
& \mathrm{C}(4)-\mathrm{Na}(1)-\mathrm{N}(8)-\mathrm{Cr}(2) \\
& 149.5(2) \\
& \mathrm{Cr}(1)-\mathrm{Na}(1)-\mathrm{N}(8)-\mathrm{Cr}(2) \\
& 158.47(16) \\
& \mathrm{Na}(2)-\mathrm{Na}(1)-\mathrm{N}(8)-\mathrm{Cr}(2) \\
& 96.05(15) \\
& \mathrm{N}(2)-\mathrm{Na}(1)-\mathrm{N}(8)-\mathrm{Na}(2) \\
& 47.14(13) \\
& N(4)-N a(1)-N(8)-N a(2) \\
& -99.23(12) \\
& \mathrm{N}(7)-\mathrm{Na}(1)-\mathrm{N}(8)-\mathrm{Na}(2) \\
& 63.4(2) \\
& \mathrm{C}(3)-\mathrm{Na}(1)-\mathrm{N}(8)-\mathrm{Na}(2) \\
& 67.95(18) \\
& \mathrm{C}(39)-\mathrm{Na}(1)-\mathrm{N}(8)-\mathrm{Na}(2) \\
& -71.78(13) \\
& \mathrm{C}(54)-\mathrm{Na}(1)-\mathrm{N}(8)-\mathrm{Na}(2) \\
& -166.89(15) \\
& \mathrm{C}(4)-\mathrm{Na}(1)-\mathrm{N}(8)-\mathrm{Na}(2) \\
& 53.5(3) \\
& \mathrm{Cr}(2)-\mathrm{Na}(1)-\mathrm{N}(8)-\mathrm{Na}(2) \\
& -96.05(15) \\
& \mathrm{Cr}(1)-\mathrm{Na}(1)-\mathrm{N}(8)-\mathrm{Na}(2) \\
& 62.42(8) \\
& N(3)-\mathrm{Na}(2)-\mathrm{N}(8)-\mathrm{N}(7) \\
& 10.8(2) \\
& \mathrm{N}(5)-\mathrm{Na}(2)-\mathrm{N}(8)-\mathrm{N}(7) \\
& 161.6(3)
\end{aligned}
$$




$$
\begin{aligned}
& \mathrm{C}(44)-\mathrm{Na}(2)-\mathrm{N}(8)-\mathrm{N}(7) \\
& -175.2(2) \\
& \mathrm{C}(8)-\mathrm{Na}(2)-\mathrm{N}(8)-\mathrm{N}(7) \\
& 42.6(3) \\
& \mathrm{C}(43)-\mathrm{Na}(2)-\mathrm{N}(8)-\mathrm{N}(7) \\
& 176.8(2) \\
& \mathrm{C}(32)-\mathrm{Na}(2)-\mathrm{N}(8)-\mathrm{N}(7) \\
& -77.0(3) \\
& \mathrm{Cr}(1)-\mathrm{Na}(2)-\mathrm{N}(8)-\mathrm{N}(7) \\
& 10.39(19) \\
& \mathrm{C}(40)-\mathrm{Na}(2)-\mathrm{N}(8)-\mathrm{N}(7) \\
& 135.2(2) \\
& \mathrm{Cr}(2)-\mathrm{Na}(2)-\mathrm{N}(8)-\mathrm{N}(7) \\
& 173.9(3) \\
& \mathrm{Na}(1)-\mathrm{Na}(2)-\mathrm{N}(8)-\mathrm{N}(7) \\
& 79.7(2) \\
& \mathrm{N}(7)-\mathrm{Na}(2)-\mathrm{N}(8)-\mathrm{Cr}(2) \\
& \mathrm{N}(3)-\mathrm{Na}(2)-\mathrm{N}(8)-\mathrm{Cr}(2) \\
& \mathrm{N}(5)-\mathrm{Na}(2)-\mathrm{N}(8)-\mathrm{Cr}(2) \\
& \mathrm{C}(44)-\mathrm{Na}(2)-\mathrm{N}(8)-\mathrm{Cr}(2) \\
& \mathrm{C}(8)-\mathrm{Na}(2)-\mathrm{N}(8)-\mathrm{Cr}(2) \\
& \mathrm{C}(43)-\mathrm{Na}(2)-\mathrm{N}(8)-\mathrm{Cr}(2) \\
& \mathrm{C}(32)-\mathrm{Na}(2)-\mathrm{N}(8)-\mathrm{Cr}(2) \\
& \mathrm{Cr}(1)-\mathrm{Na}(2)-\mathrm{N}(8)-\mathrm{Cr}(2) \\
& \mathrm{C}(4 \odot)-\mathrm{Na}(2)-\mathrm{N}(8)-\mathrm{Cr}(2) \\
& \mathrm{Na}(1)-\mathrm{Na}(2)-\mathrm{N}(8)-\mathrm{Cr}(2) \\
& \mathrm{N}(7)-\mathrm{Na}(2)-\mathrm{N}(8)-\mathrm{Na}(1) \\
& \mathrm{N}(3)-\mathrm{Na}(2)-\mathrm{N}(8)-\mathrm{Na}(1) \\
& \mathrm{N}(5)-\mathrm{Na}(2)-\mathrm{N}(8)-\mathrm{Na}(1) \\
& \mathrm{C}(44)-\mathrm{Na}(2)-\mathrm{N}(8)-\mathrm{Na}(1) \\
& \mathrm{C}(8)-\mathrm{Na}(2)-\mathrm{N}(8)-\mathrm{Na}(1) \\
& \mathrm{C}(43)-\mathrm{Na}(2)-\mathrm{N}(8)-\mathrm{Na}(1) \\
& \mathrm{C}(32)-\mathrm{Na}(2)-\mathrm{N}(8)-\mathrm{Na}(1) \\
& \mathrm{Cr}(1)-\mathrm{Na}(2)-\mathrm{N}(8)-\mathrm{Na}(1) \\
& \mathrm{C}(4 \odot)-\mathrm{Na}(2)-\mathrm{N}(8)-\mathrm{Na}(1) \\
& \mathrm{Cr}(2)-\mathrm{Na}(2)-\mathrm{N}(8)-\mathrm{Na}(1) \\
& N(7)-\operatorname{Cr}(1)-0(1)-C(37) \\
& -173.9(3) \\
& -163.04(15) \\
& -12.30(13) \\
& \text { 10.96(16) } \\
& -131.28(16) \\
& \text { 2.9(2) } \\
& \text { 109.1(2) } \\
& -163.50(16) \\
& -38.70(14) \\
& -94.17(16) \\
& -79.7(2) \\
& -68.87(14) \\
& 81.88(13) \\
& 105.13(14) \\
& -37.10(17) \\
& 97.04(15) \\
& -156.71(18) \\
& -69.32(9) \\
& 55.48(12) \\
& 94.17(16) \\
& 13.0(4)
\end{aligned}
$$




$$
\begin{aligned}
& N(2)-\operatorname{Cr}(1)-0(1)-C(37) \\
& -169.7(4) \\
& N(1)-\operatorname{Cr}(1)-0(1)-C(37) \\
& -98.5(4) \\
& \mathrm{N}(3)-\operatorname{Cr}(1)-0(1)-\mathrm{C}(37) \\
& 122.4(4) \\
& \mathrm{Na}(2)-\mathrm{Cr}(1)-0(1)-\mathrm{C}(37) \\
& 68.7(4) \\
& \mathrm{Na}(1)-\mathrm{Cr}(1)-\mathrm{O}(1)-\mathrm{C}(37) \\
& -19.1(5) \\
& N(7)-\operatorname{Cr}(1)-0(1)-C(34) \\
& \text { 174.6(3) } \\
& N(2)-\operatorname{Cr}(1)-0(1)-C(34) \\
& -8 . \odot(5) \\
& N(1)-\operatorname{Cr}(1)-0(1)-C(34) \\
& 63.2(4) \\
& \mathrm{N}(3)-\mathrm{Cr}(1)-0(1)-\mathrm{C}(34) \\
& -75.9(4) \\
& \mathrm{Na}(2)-\mathrm{Cr}(1)-\mathrm{O}(1)-\mathrm{C}(34) \\
& -129.7(3) \\
& \mathrm{Na}(1)-\mathrm{Cr}(1)-0(1)-\mathrm{C}(34) \\
& 142.6(3) \\
& \mathrm{N}(8)-\operatorname{Cr}(2)-\mathrm{O}(2)-\mathrm{C}(74) \\
& 28.3(4) \\
& \mathrm{N}(5)-\mathrm{Cr}(2)-0(2)-\mathrm{C}(74) \\
& -155.5(4) \\
& N(6)-\operatorname{Cr}(2)-O(2)-C(74) \\
& -83.4(4) \\
& \mathrm{N}(4)-\mathrm{Cr}(2)-0(2)-\mathrm{C}(74) \\
& 136.6(4) \\
& \mathrm{Na}(1)-\mathrm{Cr}(2)-\mathrm{O}(2)-\mathrm{C}(74) \\
& 82.7(4) \\
& \mathrm{Na}(2)-\mathrm{Cr}(2)-\mathrm{O}(2)-\mathrm{C}(74) \\
& \text { 2. } \odot(5) \\
& \mathrm{N}(8)-\mathrm{Cr}(2)-\mathrm{O}(2)-\mathrm{C}(71) \\
& -178 . \odot(3) \\
& N(5)-\operatorname{Cr}(2)-0(2)-C(71) \\
& -1.9(5) \\
& \mathrm{N}(6)-\mathrm{Cr}(2)-0(2)-\mathrm{C}(71) \\
& 70.3(4) \\
& \mathrm{N}(4)-\mathrm{Cr}(2)-0(2)-\mathrm{C}(71) \\
& -69.7(3) \\
& \mathrm{Na}(1)-\mathrm{Cr}(2)-\mathrm{O}(2)-\mathrm{C}(71) \\
& -123.6(3) \\
& \mathrm{Na}(2)-\mathrm{Cr}(2)-\mathrm{O}(2)-\mathrm{C}(71) \\
& 155.7(3) \\
& C(10)-N(1)-C(2)-C(3) \\
& -169.3(4) \\
& \mathrm{Cr}(1)-\mathrm{N}(1)-\mathrm{C}(2)-\mathrm{C}(3) \\
& 13.8(5) \\
& C(10)-N(1)-C(2)-C(1) \\
& 12.8(6) \\
& \mathrm{Cr}(1)-\mathrm{N}(1)-\mathrm{C}(2)-\mathrm{C}(1) \\
& -164.1(4) \\
& C(7)-N(2)-C(3)-C(2) \\
& 166.3(4)
\end{aligned}
$$




$$
\begin{aligned}
& C r(1)-N(2)-C(3)-C(2) \\
& -8.2(5) \\
& \mathrm{Na}(1)-\mathrm{N}(2)-\mathrm{C}(3)-\mathrm{C}(2) \\
& -91.9(3) \\
& \mathrm{C}(7)-\mathrm{N}(2)-\mathrm{C}(3)-\mathrm{C}(4) \\
& -13.2(6) \\
& \mathrm{Cr}(1)-\mathrm{N}(2)-\mathrm{C}(3)-\mathrm{C}(4) \\
& 172.3(3) \\
& \mathrm{Na}(1)-\mathrm{N}(2)-\mathrm{C}(3)-\mathrm{C}(4) \\
& 88.6(4) \\
& \mathrm{C}(7)-\mathrm{N}(2)-\mathrm{C}(3)-\mathrm{Na}(1) \\
& -101.8(4) \\
& \mathrm{Cr}(1)-\mathrm{N}(2)-\mathrm{C}(3)-\mathrm{Na}(1) \\
& \text { 83. 8(2) } \\
& \mathrm{N}(1)-\mathrm{C}(2)-\mathrm{C}(3)-\mathrm{N}(2) \\
& -4.3(5) \\
& \mathrm{C}(1)-\mathrm{C}(2)-\mathrm{C}(3)-\mathrm{N}(2) \\
& N(1)-C(2)-C(3)-C(4) \\
& C(1)-C(2)-C(3)-C(4) \\
& \mathrm{N}(1)-\mathrm{C}(2)-\mathrm{C}(3)-\mathrm{Na}(1) \\
& C(1)-C(2)-C(3)-\mathrm{Na}(1) \\
& \mathrm{N}(8)-\mathrm{Na}(1)-\mathrm{C}(3)-\mathrm{N}(2) \\
& \mathrm{N}(4)-\mathrm{Na}(1)-\mathrm{C}(3)-\mathrm{N}(2) \\
& \mathrm{N}(7)-\mathrm{Na}(1)-\mathrm{C}(3)-\mathrm{N}(2) \\
& \mathrm{C}(39)-\mathrm{Na}(1)-\mathrm{C}(3)-\mathrm{N}(2) \\
& \mathrm{C}(54)-\mathrm{Na}(1)-\mathrm{C}(3)-\mathrm{N}(2) \\
& \mathrm{C}(4)-\mathrm{Na}(1)-\mathrm{C}(3)-\mathrm{N}(2) \\
& \mathrm{Cr}(2)-\mathrm{Na}(1)-\mathrm{C}(3)-\mathrm{N}(2) \\
& \mathrm{Cr}(1)-\mathrm{Na}(1)-\mathrm{C}(3)-\mathrm{N}(2) \\
& \mathrm{Na}(2)-\mathrm{Na}(1)-\mathrm{C}(3)-\mathrm{N}(2) \\
& \mathrm{N}(8)-\mathrm{Na}(1)-\mathrm{C}(3)-\mathrm{C}(2) \\
& \mathrm{N}(2)-\mathrm{Na}(1)-\mathrm{C}(3)-\mathrm{C}(2) \\
& \mathrm{N}(4)-\mathrm{Na}(1)-\mathrm{C}(3)-\mathrm{C}(2) \\
& \mathrm{N}(7)-\mathrm{Na}(1)-\mathrm{C}(3)-\mathrm{C}(2) \\
& \mathrm{C}(39)-\mathrm{Na}(1)-\mathrm{C}(3)-\mathrm{C}(2) \\
& \mathrm{C}(54)-\mathrm{Na}(1)-\mathrm{C}(3)-\mathrm{C}(2) \\
& \mathrm{C}(4)-\mathrm{Na}(1)-\mathrm{C}(3)-\mathrm{C}(2) \\
& 173.6(4) \\
& \text { 175.2(4) } \\
& -6.9(8) \\
& -78.7(4) \\
& 99.3(4) \\
& -43.4(3) \\
& 94.6(5) \\
& -41.2(3) \\
& 76.7(3) \\
& -178.5(3) \\
& 117.6(4) \\
& -24.9(4) \\
& -37.7(2) \\
& 0.2(2) \\
& 67.2(3) \\
& 110.6(4) \\
& -154.8(4) \\
& 69.4(3) \\
& -172.6(3) \\
& -67.9(3) \\
& -131.8(5)
\end{aligned}
$$




$$
\begin{aligned}
& \mathrm{Cr}(2)-\mathrm{Na}(1)-\mathrm{C}(3)-\mathrm{C}(2) \quad 85.8(4) \\
& \mathrm{Cr}(1)-\mathrm{Na}(1)-\mathrm{C}(3)-\mathrm{C}(2) \quad 73.0(3) \\
& \mathrm{Na}(2)-\mathrm{Na}(1)-\mathrm{C}(3)-\mathrm{C}(2) \quad 110.8(3) \\
& \mathrm{N}(8)-\mathrm{Na}(1)-\mathrm{C}(3)-\mathrm{C}(4) \quad-161.0(3) \\
& \mathrm{N}(2)-\mathrm{Na}(1)-\mathrm{C}(3)-\mathrm{C}(4) \quad-117.6(4) \\
& \mathrm{N}(4)-\mathrm{Na}(1)-\mathrm{C}(3)-\mathrm{C}(4) \quad-23.0(6) \\
& \mathrm{N}(7)-\mathrm{Na}(1)-\mathrm{C}(3)-\mathrm{C}(4) \quad-158.8(3) \\
& \mathrm{C}(39)-\mathrm{Na}(1)-\mathrm{C}(3)-\mathrm{C}(4) \quad-40.8(4) \\
& \mathrm{C}(54)-\mathrm{Na}(1)-\mathrm{C}(3)-\mathrm{C}(4) \quad 63.9(3) \\
& \mathrm{Cr}(2)-\mathrm{Na}(1)-\mathrm{C}(3)-\mathrm{C}(4) \quad-142.4(3) \\
& \mathrm{Cr}(1)-\mathrm{Na}(1)-\mathrm{C}(3)-\mathrm{C}(4) \quad-155.3(4) \\
& \mathrm{Na}(2)-\mathrm{Na}(1)-\mathrm{C}(3)-\mathrm{C}(4) \quad-117.4(3) \\
& N(2)-C(3)-C(4)-C(5) \quad 3.7(7) \\
& C(2)-C(3)-C(4)-C(5) \quad-175.7(5) \\
& \mathrm{Na}(1)-\mathrm{C}(3)-\mathrm{C}(4)-\mathrm{C}(5) \quad 77.6(5) \\
& \mathrm{N}(2)-\mathrm{C}(3)-\mathrm{C}(4)-\mathrm{Na}(1) \quad-73.9(3) \\
& \mathrm{C}(2)-\mathrm{C}(3)-\mathrm{C}(4)-\mathrm{Na}(1) \quad 106.7(5) \\
& \mathrm{N}(8)-\mathrm{Na}(1)-\mathrm{C}(4)-\mathrm{C}(5) \quad-93.5(4) \\
& \mathrm{N}(2)-\mathrm{Na}(1)-\mathrm{C}(4)-\mathrm{C}(5) \quad-85.1(4) \\
& \mathrm{N}(4)-\mathrm{Na}(1)-\mathrm{C}(4)-\mathrm{C}(5) \quad 47.3(4) \\
& \mathrm{N}(7)-\mathrm{Na}(1)-\mathrm{C}(4)-\mathrm{C}(5) \quad-98.8(3) \\
& \mathrm{C}(3)-\mathrm{Na}(1)-\mathrm{C}(4)-\mathrm{C}(5) \quad-122.4(5) \\
& \mathrm{C}(39)-\mathrm{Na}(1)-\mathrm{C}(4)-\mathrm{C}(5) \quad 26.3(4) \\
& \mathrm{C}(54)-\mathrm{Na}(1)-\mathrm{C}(4)-\mathrm{C}(5) \quad 120.0(3) \\
& \mathrm{Cr}(2)-\mathrm{Na}(1)-\mathrm{C}(4)-\mathrm{C}(5) \quad-22.3(6) \\
& \mathrm{Cr}(1)-\mathrm{Na}(1)-\mathrm{C}(4)-\mathrm{C}(5) \quad-101.4(3) \\
& \mathrm{Na}(2)-\mathrm{Na}(1)-\mathrm{C}(4)-\mathrm{C}(5) \quad-55.0(3) \\
& \mathrm{N}(8)-\mathrm{Na}(1)-\mathrm{C}(4)-\mathrm{C}(3) \quad 28.9(4)
\end{aligned}
$$




$$
\begin{aligned}
& \mathrm{N}(2)-\mathrm{Na}(1)-\mathrm{C}(4)-\mathrm{C}(3) \quad 37.3(3) \\
& \mathrm{N}(4)-\mathrm{Na}(1)-\mathrm{C}(4)-\mathrm{C}(3) \quad 169.7 \text { (3) } \\
& \mathrm{N}(7)-\mathrm{Na}(1)-\mathrm{C}(4)-\mathrm{C}(3) \quad 23.6(4) \\
& \mathrm{C}(39)-\mathrm{Na}(1)-\mathrm{C}(4)-\mathrm{C}(3) \quad 148.7 \text { (3) } \\
& \mathrm{C}(54)-\mathrm{Na}(1)-\mathrm{C}(4)-\mathrm{C}(3) \quad-117.6(3) \\
& \begin{array}{ll}
\mathrm{Cr}(2)-\mathrm{Na}(1)-\mathrm{C}(4)-\mathrm{C}(3) & 100.0(5)
\end{array} \\
& \mathrm{Cr}(1)-\mathrm{Na}(1)-\mathrm{C}(4)-\mathrm{C}(3) \quad 20.9 \text { (3) } \\
& \mathrm{Na}(2)-\mathrm{Na}(1)-\mathrm{C}(4)-\mathrm{C}(3) \quad 67.3(3) \\
& \mathrm{C}(3)-\mathrm{C}(4)-\mathrm{C}(5)-\mathrm{C}(6) \quad 2.3(8) \\
& \mathrm{Na}(1)-\mathrm{C}(4)-\mathrm{C}(5)-\mathrm{C}(6) \quad 57.4(5) \\
& C(4)-C(5)-C(6)-C(7) \quad 0.7(7) \\
& \mathrm{C}(3)-\mathrm{N}(2)-\mathrm{C}(7)-\mathrm{C}(8) \quad-160.6(4) \\
& \operatorname{Cr}(1)-\mathrm{N}(2)-\mathrm{C}(7)-\mathrm{C}(8) \quad 13.9(5) \\
& \mathrm{Na}(1)-\mathrm{N}(2)-\mathrm{C}(7)-\mathrm{C}(8) \quad 115 . \odot(3) \\
& \mathrm{C}(3)-\mathrm{N}(2)-\mathrm{C}(7)-\mathrm{C}(6) \quad 16.3(6) \\
& \operatorname{Cr}(1)-\mathrm{N}(2)-\mathrm{C}(7)-\mathrm{C}(6) \quad-169.2(3) \\
& \mathrm{Na}(1)-\mathrm{N}(2)-\mathrm{C}(7)-\mathrm{C}(6) \quad-68.2(4) \\
& C(5)-C(6)-C(7)-C(8) \quad 166.7(5) \\
& C(5)-C(6)-C(7)-N(2) \quad-9.6(6) \\
& \mathrm{N}(2)-\mathrm{C}(7)-\mathrm{C}(8)-\mathrm{N}(3) \quad 0.7(6) \\
& \mathrm{C}(6)-\mathrm{C}(7)-\mathrm{C}(8)-\mathrm{N}(3) \quad-175.8(4) \\
& \mathrm{N}(2)-\mathrm{C}(7)-\mathrm{C}(8)-\mathrm{C}(9) \quad-176.6(4) \\
& \mathrm{C}(6)-\mathrm{C}(7)-\mathrm{C}(8)-\mathrm{C}(9) \quad 6.9(8) \\
& \mathrm{N}(2)-\mathrm{C}(7)-\mathrm{C}(8)-\mathrm{Na}(2) \quad-70.5(4) \\
& \mathrm{C}(6)-\mathrm{C}(7)-\mathrm{C}(8)-\mathrm{Na}(2) \quad 113 . \odot(4) \\
& \mathrm{C}(22)-\mathrm{N}(3)-\mathrm{C}(8)-\mathrm{C}(7) \quad 141.9(4) \\
& \operatorname{Cr}(1)-\mathrm{N}(3)-\mathrm{C}(8)-\mathrm{C}(7) \quad-13.4(5) \\
& \mathrm{Na}(2)-\mathrm{N}(3)-\mathrm{C}(8)-\mathrm{C}(7) \quad-91.6(4) \\
& C(22)-N(3)-C(8)-C(9) \quad-40.8(6)
\end{aligned}
$$




$$
\begin{aligned}
& \operatorname{Cr}(1)-N(3)-C(8)-C(9) \quad 163.9(4) \\
& \mathrm{Na}(2)-\mathrm{N}(3)-\mathrm{C}(8)-\mathrm{C}(9) \quad 85.7(4) \\
& \mathrm{C}(22)-\mathrm{N}(3)-\mathrm{C}(8)-\mathrm{Na}(2) \quad-126.5(3) \\
& \begin{array}{ll}
\mathrm{Cr}(1)-\mathrm{N}(3)-\mathrm{C}(8)-\mathrm{Na}(2) & 78.22(19)
\end{array} \\
& \mathrm{N}(7)-\mathrm{Na}(2)-\mathrm{C}(8)-\mathrm{C}(7) \quad 53.8(3) \\
& \begin{array}{ll}
\mathrm{N}(3)-\mathrm{Na}(2)-\mathrm{C}(8)-\mathrm{C}(7) & 112.9(4)
\end{array} \\
& \mathrm{N}(5)-\mathrm{Na}(2)-\mathrm{C}(8)-\mathrm{C}(7) \quad-38.5(4) \\
& \mathrm{C}(44)-\mathrm{Na}(2)-\mathrm{C}(8)-\mathrm{C}(7) \quad-76.5(4) \\
& \mathrm{N}(8)-\mathrm{Na}(2)-\mathrm{C}(8)-\mathrm{C}(7) \quad 35.3(3) \\
& \mathrm{C}(43)-\mathrm{Na}(2)-\mathrm{C}(8)-\mathrm{C}(7) \quad-97.8(3) \\
& \mathrm{C}(32)-\mathrm{Na}(2)-\mathrm{C}(8)-\mathrm{C}(7) \quad 176.6(3) \\
& \mathrm{Cr}(1)-\mathrm{Na}(2)-\mathrm{C}(8)-\mathrm{C}(7) \quad 68.1(3) \\
& \mathrm{C}(40)-\mathrm{Na}(2)-\mathrm{C}(8)-\mathrm{C}(7) \quad-41.5(3) \\
& \mathrm{Cr}(2)-\mathrm{Na}(2)-\mathrm{C}(8)-\mathrm{C}(7) \quad 4.8(4) \\
& \mathrm{Na}(1)-\mathrm{Na}(2)-\mathrm{C}(8)-\mathrm{C}(7) \quad 11.2(3) \\
& \mathrm{N}(7)-\mathrm{Na}(2)-\mathrm{C}(8)-\mathrm{N}(3) \quad-59.2(2) \\
& \mathrm{N}(5)-\mathrm{Na}(2)-\mathrm{C}(8)-\mathrm{N}(3) \quad-151.4(2) \\
& \mathrm{C}(44)-\mathrm{Na}(2)-\mathrm{C}(8)-\mathrm{N}(3) \quad 170.5(2) \\
& \mathrm{N}(8)-\mathrm{Na}(2)-\mathrm{C}(8)-\mathrm{N}(3) \quad-77.6(3) \\
& \mathrm{C}(43)-\mathrm{Na}(2)-\mathrm{C}(8)-\mathrm{N}(3) \quad 149.2(2) \\
& \begin{array}{ll}
\mathrm{C}(32)-\mathrm{Na}(2)-\mathrm{C}(8)-\mathrm{N}(3) & 63.7(3)
\end{array} \\
& \mathrm{Cr}(1)-\mathrm{Na}(2)-\mathrm{C}(8)-\mathrm{N}(3) \quad-44.8(2) \\
& \mathrm{C}(40)-\mathrm{Na}(2)-\mathrm{C}(8)-\mathrm{N}(3) \quad-154.4(2) \\
& \mathrm{Cr}(2)-\mathrm{Na}(2)-\mathrm{C}(8)-\mathrm{N}(3) \quad-108.2(2) \\
& \mathrm{Na}(1)-\mathrm{Na}(2)-\mathrm{C}(8)-\mathrm{N}(3) \quad-101.7(2) \\
& N(7)-N a(2)-C(8)-C(9) \quad-179.8(3) \\
& \mathrm{N}(3)-\mathrm{Na}(2)-\mathrm{C}(8)-\mathrm{C}(9) \quad-120.7(4) \\
& \mathrm{N}(5)-\mathrm{Na}(2)-\mathrm{C}(8)-\mathrm{C}(9) \quad 87.9(3)
\end{aligned}
$$




$$
\begin{aligned}
& \mathrm{C}(44)-\mathrm{Na}(2)-\mathrm{C}(8)-\mathrm{C}(9) \quad 49.8(4) \\
& \mathrm{N}(8)-\mathrm{Na}(2)-\mathrm{C}(8)-\mathrm{C}(9) \quad 161.7(3) \\
& \mathrm{C}(43)-\mathrm{Na}(2)-\mathrm{C}(8)-\mathrm{C}(9) \quad 28.6(3) \\
& \mathrm{C}(32)-\mathrm{Na}(2)-\mathrm{C}(8)-\mathrm{C}(9) \quad-57.0(3) \\
& \mathrm{Cr}(1)-\mathrm{Na}(2)-\mathrm{C}(8)-\mathrm{C}(9) \quad-165.5(3) \\
& \mathrm{C}(40)-\mathrm{Na}(2)-\mathrm{C}(8)-\mathrm{C}(9) \quad 84.9 \text { (3) } \\
& \operatorname{Cr}(2)-\mathrm{Na}(2)-\mathrm{C}(8)-\mathrm{C}(9) \quad 131.2(3) \\
& \mathrm{Na}(1)-\mathrm{Na}(2)-\mathrm{C}(8)-\mathrm{C}(9) \quad 137.6(3) \\
& \mathrm{C}(2)-\mathrm{N}(1)-\mathrm{C}(10)-\mathrm{C}(15) \quad-105.3(5) \\
& \begin{array}{ll}
\mathrm{Cr}(1)-\mathrm{N}(1)-\mathrm{C}(10)-\mathrm{C}(15) & 70.8(5)
\end{array} \\
& \mathrm{C}(2)-\mathrm{N}(1)-\mathrm{C}(10)-\mathrm{C}(11) \quad 74.4(5) \\
& \operatorname{Cr}(1)-N(1)-C(10)-C(11) \quad-109.4(4) \\
& C(15)-C(10)-C(11)-C(12) \quad 5.9(7) \\
& \mathrm{N}(1)-\mathrm{C}(10)-\mathrm{C}(11)-\mathrm{C}(12) \quad-173.8(4) \\
& C(15)-C(10)-C(11)-C(16) \quad-173.1(4) \\
& \mathrm{N}(1)-\mathrm{C}(10)-\mathrm{C}(11)-\mathrm{C}(16) \quad 7.2(7) \\
& C(10)-C(11)-C(12)-C(13) \quad-3 . \odot(8) \\
& C(16)-C(11)-C(12)-C(13) \quad 176.1(5) \\
& C(11)-C(12)-C(13)-C(14) \quad-0.6(9) \\
& C(12)-C(13)-C(14)-C(15) \quad 1.5(9) \\
& C(13)-C(14)-C(15)-C(10) \quad 1.4(8) \\
& C(13)-C(14)-C(15)-C(19) \quad 178.5(5) \\
& C(11)-C(10)-C(15)-C(14) \quad-5.2(7) \\
& N(1)-C(10)-C(15)-C(14) \quad 174.6(4) \\
& C(11)-C(10)-C(15)-C(19) \quad 177.8(4) \\
& \mathrm{N}(1)-\mathrm{C}(10)-\mathrm{C}(15)-\mathrm{C}(19) \quad-2.4(7) \\
& C(12)-C(11)-C(16)-C(17) \quad 76.2(7) \\
& C(10)-C(11)-C(16)-C(17) \quad-104.7(6) \\
& C(12)-C(11)-C(16)-C(18) \quad-45.9(6)
\end{aligned}
$$




$$
\begin{aligned}
& C(10)-C(11)-C(16)-C(18) \quad 133.1(5) \\
& C(14)-C(15)-C(19)-C(21) \quad 28.7(7) \\
& C(10)-C(15)-C(19)-C(21) \quad-154.4(5) \\
& C(14)-C(15)-C(19)-C(20) \quad-96.9(6) \\
& C(10)-C(15)-C(19)-C(20) \quad 80.1(6) \\
& \mathrm{C}(8)-\mathrm{N}(3)-\mathrm{C}(22)-\mathrm{C}(27) \quad 121.4(5) \\
& \mathrm{Cr}(1)-\mathrm{N}(3)-\mathrm{C}(22)-\mathrm{C}(27) \quad-88 . \odot(5) \\
& \mathrm{Na}(2)-\mathrm{N}(3)-\mathrm{C}(22)-\mathrm{C}(27) \quad 25.6(6) \\
& \mathrm{C}(8)-\mathrm{N}(3)-\mathrm{C}(22)-\mathrm{C}(23) \quad-59.3(5) \\
& \operatorname{Cr}(1)-\mathrm{N}(3)-\mathrm{C}(22)-\mathrm{C}(23) \quad 91.3(5) \\
& \mathrm{Na}(2)-\mathrm{N}(3)-\mathrm{C}(22)-\mathrm{C}(23) \quad-155.1 \text { (3) } \\
& C(27)-C(22)-C(23)-C(24) \quad-3.5(7) \\
& \mathrm{N}(3)-\mathrm{C}(22)-\mathrm{C}(23)-\mathrm{C}(24) \quad 177.2(4) \\
& C(27)-C(22)-C(23)-C(28) \quad 173.9(4) \\
& \mathrm{N}(3)-\mathrm{C}(22)-\mathrm{C}(23)-\mathrm{C}(28) \quad-5.5(7) \\
& C(22)-C(23)-C(24)-C(25) \quad 0.7(7) \\
& \mathrm{C}(28)-\mathrm{C}(23)-\mathrm{C}(24)-\mathrm{C}(25) \quad-176.8(5) \\
& C(23)-C(24)-C(25)-C(26) \quad 2.2(8) \\
& C(24)-C(25)-C(26)-C(27) \quad-2.3(8) \\
& C(25)-C(26)-C(27)-C(22) \quad-0.5(8) \\
& C(25)-C(26)-C(27)-C(31) \quad-178.9(5) \\
& \mathrm{C}(23)-\mathrm{C}(22)-\mathrm{C}(27)-\mathrm{C}(26) \quad 3.4(7) \\
& \mathrm{N}(3)-\mathrm{C}(22)-\mathrm{C}(27)-\mathrm{C}(26) \quad-177.2(4) \\
& C(23)-C(22)-C(27)-C(31) \quad-178.3(4) \\
& \mathrm{N}(3)-\mathrm{C}(22)-\mathrm{C}(27)-\mathrm{C}(31) \quad 1.0(7) \\
& C(24)-C(23)-C(28)-C(30) \quad 51.3(6) \\
& C(22)-C(23)-C(28)-C(30) \quad-126.0(5) \\
& C(24)-C(23)-C(28)-C(29) \quad-71.8(6)
\end{aligned}
$$




$$
\begin{aligned}
& C(22)-C(23)-C(28)-C(29) \\
& 110.8(5) \\
& C(26)-C(27)-C(31)-C(33) \\
& -34.6(7) \\
& C(22)-C(27)-C(31)-C(33) \\
& 147.2(5) \\
& C(26)-C(27)-C(31)-C(32) \\
& 89.2(6) \\
& C(22)-C(27)-C(31)-C(32) \\
& -89 . \odot(6) \\
& \mathrm{C}(27)-\mathrm{C}(31)-\mathrm{C}(32)-\mathrm{Na}(2) \\
& 95.8(4) \\
& \mathrm{C}(33)-\mathrm{C}(31)-\mathrm{C}(32)-\mathrm{Na}(2) \\
& \mathrm{N}(7)-\mathrm{Na}(2)-\mathrm{C}(32)-\mathrm{C}(31) \\
& -138.8(4) \\
& 20.5(4) \\
& \mathrm{N}(3)-\mathrm{Na}(2)-\mathrm{C}(32)-\mathrm{C}(31) \\
& \mathrm{N}(5)-\mathrm{Na}(2)-\mathrm{C}(32)-\mathrm{C}(31) \\
& \mathrm{C}(44)-\mathrm{Na}(2)-\mathrm{C}(32)-\mathrm{C}(31) \\
& \mathrm{C}(8)-\mathrm{Na}(2)-\mathrm{C}(32)-\mathrm{C}(31) \\
& N(8)-N a(2)-C(32)-C(31) \\
& \mathrm{C}(43)-\mathrm{Na}(2)-\mathrm{C}(32)-\mathrm{C}(31) \\
& \mathrm{Cr}(1)-\mathrm{Na}(2)-\mathrm{C}(32)-\mathrm{C}(31) \\
& \mathrm{C}(40)-\mathrm{Na}(2)-\mathrm{C}(32)-\mathrm{C}(31) \\
& \mathrm{Cr}(2)-\mathrm{Na}(2)-\mathrm{C}(32)-\mathrm{C}(31) \\
& \mathrm{Na}(1)-\mathrm{Na}(2)-\mathrm{C}(32)-\mathrm{C}(31) \\
& C(37)-0(1)-C(34)-C(35) \\
& \operatorname{Cr}(1)-0(1)-C(34)-C(35) \\
& 0(1)-C(34)-C(35)-C(36) \\
& C(34)-C(35)-C(36)-C(37) \\
& C(34)-0(1)-C(37)-C(36) \\
& \operatorname{cr}(1)-0(1)-C(37)-C(36) \\
& C(35)-C(36)-C(37)-0(1) \\
& C(47)-N(4)-C(39)-C(40) \\
& \mathrm{Cr}(2)-\mathrm{N}(4)-\mathrm{C}(39)-\mathrm{C}(40) \\
& \mathrm{Na}(1)-\mathrm{N}(4)-\mathrm{C}(39)-\mathrm{C}(40) \\
& \mathrm{C}(47)-\mathrm{N}(4)-\mathrm{C}(39)-\mathrm{C}(38) \\
& -45.6(3) \\
& 143.7(3) \\
& 145.5(3) \\
& -74.1(3) \\
& 53.3(4) \\
& 170.8(3) \\
& -14.3(3) \\
& \text { 179.5(3) } \\
& 97.1(3) \\
& -13.8(8) \\
& 7.3(6) \\
& -156.7(5) \\
& 9.5(8) \\
& -22 . \odot(9) \\
& -20.6(6) \\
& 143.7(4) \\
& 26.2(8) \\
& 144.4(4) \\
& -14.3(5) \\
& -92.9(4) \\
& -37.7(6)
\end{aligned}
$$




$$
\begin{aligned}
& \mathrm{Cr}(2)-\mathrm{N}(4)-\mathrm{C}(39)-\mathrm{C}(38) \quad 163.6(3) \\
& \mathrm{Na}(1)-\mathrm{N}(4)-\mathrm{C}(39)-\mathrm{C}(38) \quad 85.0(4) \\
& \mathrm{C}(47)-\mathrm{N}(4)-\mathrm{C}(39)-\mathrm{Na}(1) \quad-122.7 \text { (3) } \\
& \operatorname{Cr}(2)-\mathrm{N}(4)-\mathrm{C}(39)-\mathrm{Na}(1) \quad 78.64(18) \\
& \mathrm{N}(8)-\mathrm{Na}(1)-\mathrm{C}(39)-\mathrm{C}(40) \quad 53.5(3) \\
& \mathrm{N}(2)-\mathrm{Na}(1)-\mathrm{C}(39)-\mathrm{C}(40) \quad-42.9 \text { (3) } \\
& \mathrm{N}(4)-\mathrm{Na}(1)-\mathrm{C}(39)-\mathrm{C}(40) \quad 113.2(4) \\
& \mathrm{N}(7)-\mathrm{Na}(1)-\mathrm{C}(39)-\mathrm{C}(4 \odot) \quad 32 . \odot(3) \\
& \mathrm{C}(3)-\mathrm{Na}(1)-\mathrm{C}(39)-\mathrm{C}(40) \quad-77.7 \text { (3) } \\
& \begin{array}{l}
\mathrm{C}(54)-\mathrm{Na}(1)-\mathrm{C}(39)-\mathrm{C}(40) \\
175.3(3)
\end{array} \\
& \mathrm{C}(4)-\mathrm{Na}(1)-\mathrm{C}(39)-\mathrm{C}(40) \quad-95.9 \text { (3) } \\
& \begin{array}{ll}
\mathrm{Cr}(2)-\mathrm{Na}(1)-\mathrm{C}(39)-\mathrm{C}(40) & 69 . \odot(3)
\end{array} \\
& \begin{array}{ll}
\mathrm{Cr}(1)-\mathrm{Na}(1)-\mathrm{C}(39)-\mathrm{C}(40) & 0.1(3)
\end{array} \\
& \mathrm{Na}(2)-\mathrm{Na}(1)-\mathrm{C}(39)-\mathrm{C}(40) \quad 7.1 \text { (3) } \\
& \mathrm{N}(8)-\mathrm{Na}(1)-\mathrm{C}(39)-\mathrm{N}(4) \quad-59.7(2) \\
& \mathrm{N}(2)-\mathrm{Na}(1)-\mathrm{C}(39)-\mathrm{N}(4) \quad-156.2(2) \\
& \mathrm{N}(7)-\mathrm{Na}(1)-\mathrm{C}(39)-\mathrm{N}(4) \quad-81.3(2) \\
& \begin{array}{ll}
\mathrm{C}(3)-\mathrm{Na}(1)-\mathrm{C}(39)-\mathrm{N}(4) & 169 . \odot(2)
\end{array} \\
& \mathrm{C}(54)-\mathrm{Na}(1)-\mathrm{C}(39)-\mathrm{N}(4) \quad 62.0(2) \\
& \mathrm{C}(4)-\mathrm{Na}(1)-\mathrm{C}(39)-\mathrm{N}(4) \quad 150.8(2) \\
& \mathrm{Cr}(2)-\mathrm{Na}(1)-\mathrm{C}(39)-\mathrm{N}(4) \quad-44.28(19) \\
& \mathrm{Cr}(1)-\mathrm{Na}(1)-\mathrm{C}(39)-\mathrm{N}(4) \quad-113.2(2) \\
& \mathrm{Na}(2)-\mathrm{Na}(1)-\mathrm{C}(39)-\mathrm{N}(4) \quad-106.1(2) \\
& \mathrm{N}(8)-\mathrm{Na}(1)-\mathrm{C}(39)-\mathrm{C}(38) \quad 178.9(3) \\
& \mathrm{N}(2)-\mathrm{Na}(1)-\mathrm{C}(39)-\mathrm{C}(38) \quad 82.5(3) \\
& \mathrm{N}(4)-\mathrm{Na}(1)-\mathrm{C}(39)-\mathrm{C}(38) \quad-121.3(4) \\
& \mathrm{N}(7)-\mathrm{Na}(1)-\mathrm{C}(39)-\mathrm{C}(38) \quad 157.4 \text { (3) } \\
& \mathrm{C}(3)-\mathrm{Na}(1)-\mathrm{C}(39)-\mathrm{C}(38) \quad 47.7(4)
\end{aligned}
$$




$$
\begin{aligned}
& \mathrm{C}(54)-\mathrm{Na}(1)-\mathrm{C}(39)-\mathrm{C}(38) \\
& -59.3(3) \\
& \mathrm{C}(4)-\mathrm{Na}(1)-\mathrm{C}(39)-\mathrm{C}(38) \\
& 29.5(3) \\
& \mathrm{Cr}(2)-\mathrm{Na}(1)-\mathrm{C}(39)-\mathrm{C}(38) \\
& -165.6(3) \\
& \mathrm{Cr}(1)-\mathrm{Na}(1)-\mathrm{C}(39)-\mathrm{C}(38) \\
& 125.5(3) \\
& \mathrm{Na}(2)-\mathrm{Na}(1)-\mathrm{C}(39)-\mathrm{C}(38) \\
& 132.5(3) \\
& \mathrm{N}(4)-\mathrm{C}(39)-\mathrm{C}(40)-\mathrm{N}(5) \\
& -0.2(5) \\
& \mathrm{C}(38)-\mathrm{C}(39)-\mathrm{C}(40)-\mathrm{N}(5) \\
& -178.1(4) \\
& \mathrm{Na}(1)-\mathrm{C}(39)-\mathrm{C}(40)-\mathrm{N}(5) \\
& -73.8(3) \\
& N(4)-C(39)-C(40)-C(41) \\
& C(38)-C(39)-C(40)-C(41) \\
& -178.1(4) \\
& \text { 4.1(7) } \\
& \mathrm{Na}(1)-\mathrm{C}(39)-\mathrm{C}(40)-\mathrm{C}(41) \\
& \mathrm{N}(4)-\mathrm{C}(39)-\mathrm{C}(40)-\mathrm{Na}(2) \\
& 108.4(5) \\
& C(38)-C(39)-C(40)-\mathrm{Na}(2) \\
& 63.2(5) \\
& -114.7(4) \\
& \mathrm{Na}(1)-\mathrm{C}(39)-\mathrm{C}(40)-\mathrm{Na}(2) \\
& -10.4(4) \\
& \mathrm{C}(44)-\mathrm{N}(5)-\mathrm{C}(40)-\mathrm{C}(39) \\
& -163.5(4) \\
& \mathrm{Cr}(2)-\mathrm{N}(5)-\mathrm{C}(40)-\mathrm{C}(39) \\
& 16.5(5) \\
& \mathrm{Na}(2)-\mathrm{N}(5)-\mathrm{C}(40)-\mathrm{C}(39) \\
& 119.4(3) \\
& \mathrm{C}(44)-\mathrm{N}(5)-\mathrm{C}(40)-\mathrm{C}(41) \\
& 14.6(6) \\
& \mathrm{Cr}(2)-\mathrm{N}(5)-\mathrm{C}(40)-\mathrm{C}(41) \\
& -165.4(3) \\
& \mathrm{Na}(2)-\mathrm{N}(5)-\mathrm{C}(40)-\mathrm{C}(41) \\
& -62.5(4) \\
& \mathrm{C}(44)-\mathrm{N}(5)-\mathrm{C}(40)-\mathrm{Na}(2) \\
& 77.1(3) \\
& \mathrm{Cr}(2)-\mathrm{N}(5)-\mathrm{C}(40)-\mathrm{Na}(2) \\
& -102.9(3) \\
& \mathrm{N}(7)-\mathrm{Na}(2)-\mathrm{C}(40)-\mathrm{C}(39) \\
& -13.4(4) \\
& \mathrm{N}(3)-\mathrm{Na}(2)-\mathrm{C}(40)-\mathrm{C}(39) \\
& 63.8(4) \\
& \mathrm{N}(5)-\mathrm{Na}(2)-\mathrm{C}(4 \odot)-\mathrm{C}(39) \\
& -94.4(4) \\
& \mathrm{C}(44)-\mathrm{Na}(2)-\mathrm{C}(40)-\mathrm{C}(39) \\
& -129.5(4) \\
& \mathrm{C}(8)-\mathrm{Na}(2)-\mathrm{C}(40)-\mathrm{C}(39) \\
& 80.1(4) \\
& \mathrm{N}(8)-\mathrm{Na}(2)-\mathrm{C}(40)-\mathrm{C}(39) \\
& -32.7(4) \\
& \mathrm{C}(43)-\mathrm{Na}(2)-\mathrm{C}(40)-\mathrm{C}(39) \\
& -165.3(4)
\end{aligned}
$$




$$
\begin{aligned}
& \mathrm{C}(32)-\mathrm{Na}(2)-\mathrm{C}(40)-\mathrm{C}(39) \quad-175.7(4) \\
& \mathrm{Cr}(1)-\mathrm{Na}(2)-\mathrm{C}(40)-\mathrm{C}(39) \quad 18.5(4) \\
& \mathrm{Cr}(2)-\mathrm{Na}(2)-\mathrm{C}(40)-\mathrm{C}(39) \quad-56.4(3) \\
& \mathrm{Na}(1)-\mathrm{Na}(2)-\mathrm{C}(40)-\mathrm{C}(39) \quad 8.8(3) \\
& \mathrm{N}(7)-\mathrm{Na}(2)-\mathrm{C}(4 \odot)-\mathrm{N}(5) \quad 81.0(3) \\
& \mathrm{N}(3)-\mathrm{Na}(2)-\mathrm{C}(40)-\mathrm{N}(5) \quad 158.3(3) \\
& \mathrm{C}(44)-\mathrm{Na}(2)-\mathrm{C}(4 \odot)-\mathrm{N}(5) \quad-35.1(2) \\
& \mathrm{C}(8)-\mathrm{Na}(2)-\mathrm{C}(40)-\mathrm{N}(5) \quad 174.5 \text { (3) } \\
& \mathrm{N}(8)-\mathrm{Na}(2)-\mathrm{C}(40)-\mathrm{N}(5) \quad 61.8(2) \\
& \begin{array}{ll}
\mathrm{C}(43)-\mathrm{Na}(2)-\mathrm{C}(40)-\mathrm{N}(5) & -70.8(3)
\end{array} \\
& \mathrm{C}(32)-\mathrm{Na}(2)-\mathrm{C}(4 \odot)-\mathrm{N}(5) \quad-81.3(4) \\
& \operatorname{Cr}(1)-\mathrm{Na}(2)-\mathrm{C}(40)-\mathrm{N}(5) \quad 112.9(2) \\
& \mathrm{Cr}(2)-\mathrm{Na}(2)-\mathrm{C}(40)-\mathrm{N}(5) \quad 38 . \Theta(2) \\
& \mathrm{Na}(1)-\mathrm{Na}(2)-\mathrm{C}(40)-\mathrm{N}(5) \quad 103.3(3) \\
& \mathrm{N}(7)-\mathrm{Na}(2)-\mathrm{C}(40)-\mathrm{C}(41) \quad-150.4(3) \\
& \mathrm{N}(3)-\mathrm{Na}(2)-\mathrm{C}(40)-\mathrm{C}(41) \quad-73.2(3) \\
& \mathrm{N}(5)-\mathrm{Na}(2)-\mathrm{C}(40)-\mathrm{C}(41) \quad 128.5(4) \\
& \mathrm{C}(44)-\mathrm{Na}(2)-\mathrm{C}(40)-\mathrm{C}(41) \quad 93.5(3) \\
& \mathrm{C}(8)-\mathrm{Na}(2)-\mathrm{C}(40)-\mathrm{C}(41) \quad-57 . \odot(3) \\
& \mathrm{N}(8)-\mathrm{Na}(2)-\mathrm{C}(40)-\mathrm{C}(41) \quad-169.7(3) \\
& \mathrm{C}(43)-\mathrm{Na}(2)-\mathrm{C}(40)-\mathrm{C}(41) \quad 57.7 \text { (3) } \\
& \mathrm{C}(32)-\mathrm{Na}(2)-\mathrm{C}(40)-\mathrm{C}(41) \quad 47.3(4) \\
& \mathrm{Cr}(1)-\mathrm{Na}(2)-\mathrm{C}(40)-\mathrm{C}(41) \quad-118.6 \text { (3) } \\
& \mathrm{Cr}(2)-\mathrm{Na}(2)-\mathrm{C}(40)-\mathrm{C}(41) \quad 166.5(3) \\
& \mathrm{Na}(1)-\mathrm{Na}(2)-\mathrm{C}(40)-\mathrm{C}(41) \quad-128.2(3) \\
& C(39)-C(40)-C(41)-C(42) \quad 169.9(4) \\
& \mathrm{N}(5)-\mathrm{C}(40)-\mathrm{C}(41)-\mathrm{C}(42) \quad-7.8(6) \\
& \mathrm{Na}(2)-\mathrm{C}(40)-\mathrm{C}(41)-\mathrm{C}(42) \quad-55.3(4)
\end{aligned}
$$




$$
\begin{aligned}
& C(40)-C(41)-C(42)-C(43) \\
& -1.1(7) \\
& C(41)-C(42)-C(43)-C(44) \\
& \text { 4. } \odot(7) \\
& \mathrm{C}(41)-\mathrm{C}(42)-\mathrm{C}(43)-\mathrm{Na}(2) \\
& 62.6(4) \\
& \mathrm{N}(7)-\mathrm{Na}(2)-\mathrm{C}(43)-\mathrm{C}(42) \\
& -105.8(3) \\
& N(3)-N a(2)-C(43)-C(42) \\
& 50.1(4) \\
& \mathrm{N}(5)-\mathrm{Na}(2)-\mathrm{C}(43)-\mathrm{C}(42) \\
& -90.8(3) \\
& \mathrm{C}(44)-\mathrm{Na}(2)-\mathrm{C}(43)-\mathrm{C}(42) \\
& -125 . \odot(4) \\
& \mathrm{C}(8)-\mathrm{Na}(2)-\mathrm{C}(43)-\mathrm{C}(42) \\
& 25.4(3) \\
& \mathrm{N}(8)-\mathrm{Na}(2)-\mathrm{C}(43)-\mathrm{C}(42) \\
& \mathrm{C}(32)-\mathrm{Na}(2)-\mathrm{C}(43)-\mathrm{C}(42) \\
& \mathrm{Cr}(1)-\mathrm{Na}(2)-\mathrm{C}(43)-\mathrm{C}(42) \\
& \mathrm{C}(4 \odot)-\mathrm{Na}(2)-\mathrm{C}(43)-\mathrm{C}(42) \\
& \mathrm{Cr}(2)-\mathrm{Na}(2)-\mathrm{C}(43)-\mathrm{C}(42) \\
& \mathrm{Na}(1)-\mathrm{Na}(2)-\mathrm{C}(43)-\mathrm{C}(42) \\
& \mathrm{N}(7)-\mathrm{Na}(2)-\mathrm{C}(43)-\mathrm{C}(44) \\
& \mathrm{N}(3)-\mathrm{Na}(2)-\mathrm{C}(43)-\mathrm{C}(44) \\
& \mathrm{N}(5)-\mathrm{Na}(2)-\mathrm{C}(43)-\mathrm{C}(44) \\
& \mathrm{C}(8)-\mathrm{Na}(2)-\mathrm{C}(43)-\mathrm{C}(44) \\
& \mathrm{N}(8)-\mathrm{Na}(2)-\mathrm{C}(43)-\mathrm{C}(44) \\
& \mathrm{C}(32)-\mathrm{Na}(2)-\mathrm{C}(43)-\mathrm{C}(44) \\
& \mathrm{Cr}(1)-\mathrm{Na}(2)-\mathrm{C}(43)-\mathrm{C}(44) \\
& \mathrm{C}(40)-\mathrm{Na}(2)-\mathrm{C}(43)-\mathrm{C}(44) \\
& \mathrm{Cr}(2)-\mathrm{Na}(2)-\mathrm{C}(43)-\mathrm{C}(44) \\
& \mathrm{Na}(1)-\mathrm{Na}(2)-\mathrm{C}(43)-\mathrm{C}(44) \\
& \mathrm{C}(40)-\mathrm{N}(5)-\mathrm{C}(44)-\mathrm{C}(43) \\
& \operatorname{Cr}(2)-N(5)-C(44)-C(43) \\
& \mathrm{Na}(2)-\mathrm{N}(5)-\mathrm{C}(44)-\mathrm{C}(43) \\
& \mathrm{C}(40)-\mathrm{N}(5)-\mathrm{C}(44)-\mathrm{C}(45) \\
& \mathrm{Cr}(2)-\mathrm{N}(5)-\mathrm{C}(44)-\mathrm{C}(45) \\
& -108.2(3) \\
& 115.6(3) \\
& -42.4(7) \\
& -57.7(3) \\
& -106.7(3) \\
& -63.0(3) \\
& \text { 19.2(4) } \\
& 175.2(3) \\
& 34.3(3) \\
& 150.5(3) \\
& 16.8(3) \\
& -119.3(3) \\
& 82.7(6) \\
& 67.3(3) \\
& 18 \cdot 3(3) \\
& 62.1(3) \\
& -11.8(6) \\
& \text { 168.1(3) } \\
& 77.4(4) \\
& \text { 169.5(4) } \\
& -10.5(5)
\end{aligned}
$$




$$
\begin{aligned}
& \mathrm{Na}(2)-\mathrm{N}(5)-\mathrm{C}(44)-\mathrm{C}(45) \quad-101.2(3) \\
& \mathrm{C}(40)-\mathrm{N}(5)-\mathrm{C}(44)-\mathrm{Na}(2) \quad-89.3(3) \\
& \mathrm{Cr}(2)-\mathrm{N}(5)-\mathrm{C}(44)-\mathrm{Na}(2) \quad 90.7(2) \\
& C(42)-C(43)-C(44)-N(5) \quad 2.3(6) \\
& \mathrm{Na}(2)-\mathrm{C}(43)-\mathrm{C}(44)-\mathrm{N}(5) \quad-69.8(3) \\
& C(42)-C(43)-C(44)-C(45) \quad-179.3(4) \\
& \begin{array}{ll}
\mathrm{Na}(2)-\mathrm{C}(43)-\mathrm{C}(44)-\mathrm{C}(45) & 108.6(5)
\end{array} \\
& \mathrm{C}(42)-\mathrm{C}(43)-\mathrm{C}(44)-\mathrm{Na}(2) \quad 72.1(4) \\
& \mathrm{N}(7)-\mathrm{Na}(2)-\mathrm{C}(44)-\mathrm{N}(5) \quad-45.9 \text { (3) } \\
& \mathrm{N}(3)-\mathrm{Na}(2)-\mathrm{C}(44)-\mathrm{N}(5) \quad 104.8(8) \\
& \mathrm{C}(8)-\mathrm{Na}(2)-\mathrm{C}(44)-\mathrm{N}(5) \quad 78.3(3) \\
& \mathrm{N}(8)-\mathrm{Na}(2)-\mathrm{C}(44)-\mathrm{N}(5) \quad-43.5(2) \\
& \begin{array}{ll}
\mathrm{C}(43)-\mathrm{Na}(2)-\mathrm{C}(44)-\mathrm{N}(5) & 121 . \odot(4)
\end{array} \\
& \mathrm{C}(32)-\mathrm{Na}(2)-\mathrm{C}(44)-\mathrm{N}(5) \quad-177.4(3) \\
& \mathrm{Cr}(1)-\mathrm{Na}(2)-\mathrm{C}(44)-\mathrm{N}(5) \quad-34.5(4) \\
& \mathrm{C}(4 \odot)-\mathrm{Na}(2)-\mathrm{C}(44)-\mathrm{N}(5) \quad 30.4(2) \\
& \mathrm{Cr}(2)-\mathrm{Na}(2)-\mathrm{C}(44)-\mathrm{N}(5) \quad-36.70(19) \\
& \mathrm{Na}(1)-\mathrm{Na}(2)-\mathrm{C}(44)-\mathrm{N}(5) \quad-3.2(2) \\
& \mathrm{N}(7)-\mathrm{Na}(2)-\mathrm{C}(44)-\mathrm{C}(43) \quad-167.0(3) \\
& \mathrm{N}(3)-\mathrm{Na}(2)-\mathrm{C}(44)-\mathrm{C}(43) \quad-16.2(9) \\
& \mathrm{N}(5)-\mathrm{Na}(2)-\mathrm{C}(44)-\mathrm{C}(43) \quad-121.0(4) \\
& \mathrm{C}(8)-\mathrm{Na}(2)-\mathrm{C}(44)-\mathrm{C}(43) \quad-42.7(4) \\
& \mathrm{N}(8)-\mathrm{Na}(2)-\mathrm{C}(44)-\mathrm{C}(43) \quad-164.5(3) \\
& \mathrm{C}(32)-\mathrm{Na}(2)-\mathrm{C}(44)-\mathrm{C}(43) \quad 61.6(3) \\
& \mathrm{Cr}(1)-\mathrm{Na}(2)-\mathrm{C}(44)-\mathrm{C}(43) \quad-155.5(3) \\
& \mathrm{C}(40)-\mathrm{Na}(2)-\mathrm{C}(44)-\mathrm{C}(43) \quad-90.7(3) \\
& \mathrm{Cr}(2)-\mathrm{Na}(2)-\mathrm{C}(44)-\mathrm{C}(43) \quad-157.7 \text { (3) } \\
& \mathrm{Na}(1)-\mathrm{Na}(2)-\mathrm{C}(44)-\mathrm{C}(43) \quad-124.2(3)
\end{aligned}
$$




$$
\begin{aligned}
& \mathrm{N}(7)-\mathrm{Na}(2)-\mathrm{C}(44)-\mathrm{C}(45) \\
& 62.8(3) \\
& \mathrm{N}(3)-\mathrm{Na}(2)-\mathrm{C}(44)-\mathrm{C}(45) \\
& -146.4(7) \\
& N(5)-N a(2)-C(44)-C(45) \\
& 108.8(4) \\
& \mathrm{C}(8)-\mathrm{Na}(2)-\mathrm{C}(44)-\mathrm{C}(45) \\
& -172.9(3) \\
& \mathrm{N}(8)-\mathrm{Na}(2)-\mathrm{C}(44)-\mathrm{C}(45) \\
& 65.3(3) \\
& \mathrm{C}(43)-\mathrm{Na}(2)-\mathrm{C}(44)-\mathrm{C}(45) \\
& -130.2(4) \\
& \mathrm{C}(32)-\mathrm{Na}(2)-\mathrm{C}(44)-\mathrm{C}(45) \\
& -68.6(3) \\
& \mathrm{Cr}(1)-\mathrm{Na}(2)-\mathrm{C}(44)-\mathrm{C}(45) \\
& 74.3(4) \\
& \mathrm{C}(4 \odot)-\mathrm{Na}(2)-\mathrm{C}(44)-\mathrm{C}(45) \\
& 139.1(4) \\
& \mathrm{Cr}(2)-\mathrm{Na}(2)-\mathrm{C}(44)-\mathrm{C}(45) \\
& 72.1(3) \\
& \mathrm{Na}(1)-\mathrm{Na}(2)-\mathrm{C}(44)-\mathrm{C}(45) \\
& 105.6(3) \\
& \mathrm{C}(59)-\mathrm{N}(6)-\mathrm{C}(45)-\mathrm{C}(44) \\
& -173.7(4) \\
& \mathrm{Cr}(2)-\mathrm{N}(6)-\mathrm{C}(45)-\mathrm{C}(44) \\
& 14.3(4) \\
& \mathrm{C}(59)-\mathrm{N}(6)-\mathrm{C}(45)-\mathrm{C}(46) \\
& 5.9(6) \\
& \operatorname{Cr}(2)-\mathrm{N}(6)-\mathrm{C}(45)-\mathrm{C}(46) \\
& N(5)-C(44)-C(45)-N(6) \\
& -166.2(4) \\
& -3.2(5) \\
& \mathrm{C}(43)-\mathrm{C}(44)-\mathrm{C}(45)-\mathrm{N}(6) \\
& 178.3(4) \\
& \mathrm{Na}(2)-\mathrm{C}(44)-\mathrm{C}(45)-\mathrm{N}(6) \\
& -81.8(4) \\
& N(5)-C(44)-C(45)-C(46) \\
& 177.2(4) \\
& C(43)-C(44)-C(45)-C(46) \\
& -1.3(7) \\
& \mathrm{Na}(2)-\mathrm{C}(44)-\mathrm{C}(45)-\mathrm{C}(46) \\
& 98.6(4) \\
& \mathrm{C}(39)-\mathrm{N}(4)-\mathrm{C}(47)-\mathrm{C}(48) \\
& 120.9(4) \\
& \mathrm{Cr}(2)-\mathrm{N}(4)-\mathrm{C}(47)-\mathrm{C}(48) \\
& -85 . \odot(5) \\
& \mathrm{Na}(1)-\mathrm{N}(4)-\mathrm{C}(47)-\mathrm{C}(48) \\
& 28.7(6) \\
& \mathrm{C}(39)-\mathrm{N}(4)-\mathrm{C}(47)-\mathrm{C}(52) \\
& -59.1(5) \\
& \operatorname{Cr}(2)-\mathrm{N}(4)-\mathrm{C}(47)-\mathrm{C}(52) \\
& 95 . \odot(5) \\
& \mathrm{Na}(1)-\mathrm{N}(4)-\mathrm{C}(47)-\mathrm{C}(52) \\
& -151.3(3) \\
& C(52)-C(47)-C(48)-C(49) \\
& \text { 3. 6(7) } \\
& N(4)-C(47)-C(48)-C(49) \\
& -176.3(4)
\end{aligned}
$$




$$
\begin{aligned}
& C(52)-C(47)-C(48)-C(53) \quad-178.8(4) \\
& \mathrm{N}(4)-\mathrm{C}(47)-\mathrm{C}(48)-\mathrm{C}(53) \quad 1.2(7) \\
& C(47)-C(48)-C(49)-C(50) \quad-0.4(8) \\
& C(53)-C(48)-C(49)-C(50) \quad-178 . \odot(5) \\
& C(48)-C(49)-C(50)-C(51) \quad-1.7(8) \\
& C(49)-C(50)-C(51)-C(52) \quad 0.5(8) \\
& C(5 \odot)-C(51)-C(52)-C(47) \quad 2.8(7) \\
& C(50)-C(51)-C(52)-C(56) \quad-176.8(5) \\
& C(48)-C(47)-C(52)-C(51) \quad-4.8(6) \\
& \mathrm{N}(4)-\mathrm{C}(47)-\mathrm{C}(52)-\mathrm{C}(51) \quad 175.2(4) \\
& \mathrm{C}(48)-\mathrm{C}(47)-\mathrm{C}(52)-\mathrm{C}(56) \quad 174.7(4) \\
& N(4)-C(47)-C(52)-C(56) \quad-5.3(7) \\
& C(49)-C(48)-C(53)-C(55) \quad-42.6(6) \\
& C(47)-C(48)-C(53)-C(55) \quad 139.8(5) \\
& \begin{array}{l}
C(49)-C(48)-C(53)-C(54) \quad 82.0(6)
\end{array} \\
& C(47)-C(48)-C(53)-C(54) \quad-95.6(5) \\
& \mathrm{C}(48)-\mathrm{C}(53)-\mathrm{C}(54)-\mathrm{Na}(1) \quad 97.2(3) \\
& \mathrm{C}(55)-\mathrm{C}(53)-\mathrm{C}(54)-\mathrm{Na}(1) \quad-137.6(4) \\
& \mathrm{N}(8)-\mathrm{Na}(1)-\mathrm{C}(54)-\mathrm{C}(53) \quad 22.0(3) \\
& N(2)-\mathrm{Na}(1)-\mathrm{C}(54)-\mathrm{C}(53) \quad 154.8 \text { (3) } \\
& \mathrm{N}(4)-\mathrm{Na}(1)-\mathrm{C}(54)-\mathrm{C}(53) \quad-43.1 \text { (3) } \\
& \mathrm{N}(7)-\mathrm{Na}(1)-\mathrm{C}(54)-\mathrm{C}(53) \quad 55.0(4) \\
& \mathrm{C}(3)-\mathrm{Na}(1)-\mathrm{C}(54)-\mathrm{C}(53) \quad 155.8(3) \\
& \mathrm{C}(39)-\mathrm{Na}(1)-\mathrm{C}(54)-\mathrm{C}(53) \quad-70.7(3) \\
& \mathrm{C}(4)-\mathrm{Na}(1)-\mathrm{C}(54)-\mathrm{C}(53) \quad 179.7 \text { (3) } \\
& \mathrm{Cr}(2)-\mathrm{Na}(1)-\mathrm{C}(54)-\mathrm{C}(53) \quad-11.3(3) \\
& \mathrm{Cr}(1)-\mathrm{Na}(1)-\mathrm{C}(54)-\mathrm{C}(53) \quad 103.7 \text { (3) } \\
& \mathrm{Na}(2)-\mathrm{Na}(1)-\mathrm{C}(54)-\mathrm{C}(53) \quad-19.3(7)
\end{aligned}
$$




$$
\begin{aligned}
& C(51)-C(52)-C(56)-C(58) \\
& 60.3(6) \\
& C(47)-C(52)-C(56)-C(58) \\
& -119.2(5) \\
& C(51)-C(52)-C(56)-C(57) \\
& -62.3(6) \\
& C(47)-C(52)-C(56)-C(57) \\
& 118.2(5) \\
& C(45)-N(6)-C(59)-C(64) \\
& -92.5(5) \\
& C r(2)-N(6)-C(59)-C(64) \\
& 78 . \odot(5) \\
& C(45)-N(6)-C(59)-C(60) \\
& 85.5(5) \\
& C r(2)-N(6)-C(59)-C(60) \\
& -104 . \odot(4) \\
& C(64)-C(59)-C(60)-C(61) \\
& 0.6(6) \\
& N(6)-C(59)-C(60)-C(61) \\
& -177.4(4) \\
& C(64)-C(59)-C(60)-C(65) \\
& 179.4(4) \\
& N(6)-C(59)-C(60)-C(65) \\
& \text { 1. 4(6) } \\
& C(59)-C(60)-C(61)-C(62) \\
& -0.1(7) \\
& C(65)-C(60)-C(61)-C(62) \\
& -178.9(5) \\
& C(60)-C(61)-C(62)-C(63) \\
& -1.3(8) \\
& C(61)-C(62)-C(63)-C(64) \\
& 2.2(8) \\
& C(60)-C(59)-C(64)-C(63) \\
& 0.2(6) \\
& N(6)-C(59)-C(64)-C(63) \\
& 178.2(4) \\
& C(60)-C(59)-C(64)-C(68) \\
& -176 . \odot(4) \\
& N(6)-C(59)-C(64)-C(68) \\
& \text { 1.9(7) } \\
& C(62)-C(63)-C(64)-C(59) \\
& -1.6(7) \\
& C(62)-C(63)-C(64)-C(68) \\
& 174.8(5) \\
& C(61)-C(60)-C(65)-C(67) \\
& 75 . \odot(5) \\
& C(59)-C(60)-C(65)-C(67) \\
& -103.8(5) \\
& C(61)-C(60)-C(65)-C(66) \\
& -46.8(6) \\
& C(59)-C(60)-C(65)-C(66) \\
& 134.4(4) \\
& C(59)-C(64)-C(68)-C(70) \\
& 105.4(6) \\
& C(63)-C(64)-C(68)-C(70) \\
& -70.9(7) \\
& C(59)-C(64)-C(68)-C(69) \\
& -133.9(5)
\end{aligned}
$$




$$
\begin{array}{lr}
C(63)-C(64)-C(68)-C(69) & 49.8(7) \\
C(74)-0(2)-C(71)-C(72) & 16.1(5) \\
C r(2)-0(2)-C(71)-C(72) & -140.7(3) \\
O(2)-C(71)-C(72)-C(73) & -30.8(6) \\
C(71)-C(72)-C(73)-C(74) & 33.7(6) \\
C(71)-0(2)-C(74)-C(73) & 5.6(6) \\
C r(2)-0(2)-C(74)-C(73) & 163.0(4) \\
C(72)-C(73)-C(74)-0(2) & -24.8(6)
\end{array}
$$

Symmetry transformations used to generate equivalent atoms: 
Complex 7 
Table S19. Crystal data and structure refinement for 7.

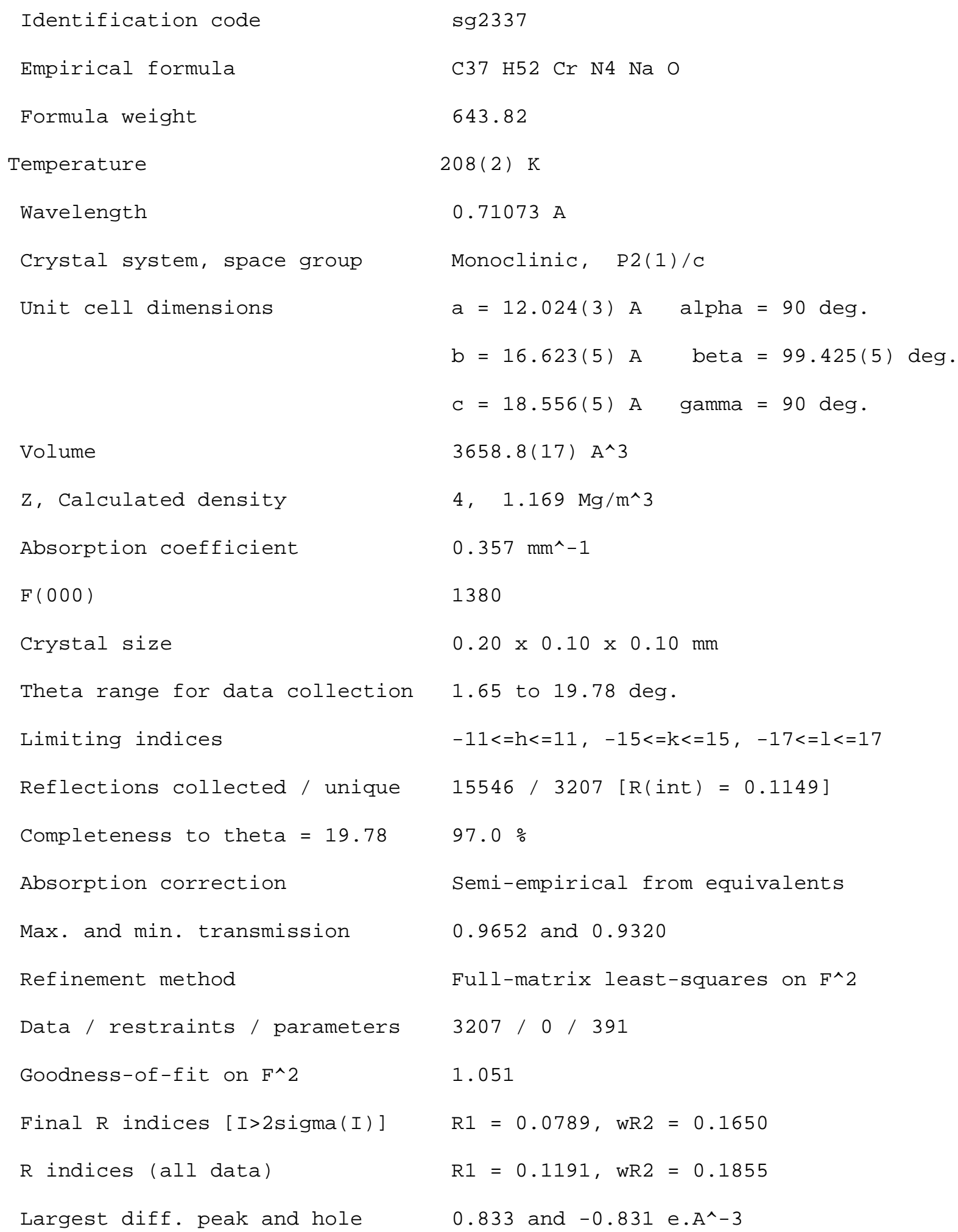


Table S20. Atomic coordinates $(x 10 \wedge 4)$ and equivalent isotropic displacement parameters $\left(A^{\wedge} 2 \times 10^{\wedge} 3\right)$ for 7 . $\mathrm{U}(\mathrm{eq})$ is defined as one third of the trace of the orthogonalized Uij tensor.

$\begin{array}{lllll}\text { x } & y & \mathrm{z} & \mathrm{u}(\mathrm{eq})\end{array}$

\begin{tabular}{|c|c|c|c|c|}
\hline $\operatorname{cr}(1)$ & $3998(1)$ & $320(1)$ & $2062(1)$ & $33(1)$ \\
\hline $\mathrm{Na}(1)$ & $6185(3)$ & $1874(2)$ & $2396(2)$ & $56(1)$ \\
\hline$N(1)$ & $2328(5)$ & $408(4)$ & $2044(3)$ & $30(2)$ \\
\hline$N(2)$ & $3491(6)$ & $-772(4)$ & $1775(3)$ & $29(2)$ \\
\hline$N(3)$ & $5449(6)$ & $-239(5)$ & $1950(4)$ & $65(2)$ \\
\hline$N(4)$ & $4522(6)$ & $1386(4)$ & $2326(4)$ & $65(2)$ \\
\hline$C(1)$ & $470(7)$ & $-314(5)$ & $1798(5)$ & $56(3)$ \\
\hline$C(2)$ & $1710(7)$ & $-275(5)$ & $1835(4)$ & $38(2)$ \\
\hline$C(3)$ & 2356 (7) & $-935(5)$ & $1677(4)$ & $32(2)$ \\
\hline$C(4)$ & $1977(7)$ & $-1721(5)$ & $1435(4)$ & $37(2)$ \\
\hline$C(5)$ & $2750(8)$ & $-2280(5)$ & $1272(5)$ & $46(2)$ \\
\hline$C(6)$ & $3900(7)$ & $-2096(5)$ & $1367(4)$ & $39(2)$ \\
\hline$C(7)$ & $4261(7)$ & $-1331(5)$ & $1629(4)$ & $33(2)$ \\
\hline$C(8)$ & $5394(7)$ & $-1031(4)$ & $1734(4)$ & $31(2)$ \\
\hline$C(9)$ & $6373(7)$ & $-1509(5)$ & $1648(5)$ & $49(2)$ \\
\hline$c(10)$ & $1687(6)$ & $1385(5)$ & $2868(5)$ & $36(2)$ \\
\hline$c(11)$ & $1219(7)$ & $2138(6)$ & $2972(5)$ & $44(2)$ \\
\hline$c(12)$ & $795(7)$ & $2629(5)$ & $2400(6)$ & $50(3)$ \\
\hline
\end{tabular}




\begin{tabular}{|c|c|c|c|c|}
\hline$c(13)$ & $847(7)$ & $2379(5)$ & $1706(6)$ & $45(2)$ \\
\hline$C(14)$ & $1324(6)$ & $1635(5)$ & $1564(5)$ & $36(2)$ \\
\hline$c(15)$ & $1748(6)$ & $1135(5)$ & $2161(5)$ & $28(2)$ \\
\hline$C(16)$ & $2121(6)$ & $856(5)$ & $3534(4)$ & $41(2)$ \\
\hline$C(17)$ & 1193 (8) & $652(6)$ & 3978 (5) & $78(3)$ \\
\hline$c(18)$ & $3132(8)$ & $1233(6)$ & $4013(5)$ & $69(3)$ \\
\hline$C(19)$ & $1439(7)$ & $1404(5)$ & $786(5)$ & $47(2)$ \\
\hline$C(20)$ & $332(10)$ & $1438(8)$ & $267(6)$ & $116(5)$ \\
\hline$C(21)$ & $2338(9)$ & $1946(7)$ & $520(6)$ & $93(4)$ \\
\hline$C(22)$ & $7139(7)$ & $259(5)$ & $2773(4)$ & $33(2)$ \\
\hline$C(23)$ & $8097(7)$ & $737(5)$ & $2873(5)$ & $41(2)$ \\
\hline$C(24)$ & $8456(7)$ & $1134(5)$ & $2309(6)$ & $47(2)$ \\
\hline$c(25)$ & $7876(7)$ & $1025(5)$ & $1609(5)$ & $4 \odot(2)$ \\
\hline$C(26)$ & $6893(7)$ & $561(5)$ & $1474(5)$ & $34(2)$ \\
\hline$C(27)$ & $6542(6)$ & $181(4)$ & $2070(5)$ & $30(2)$ \\
\hline$C(28)$ & $6775(8)$ & $-178(5)$ & $3413(5)$ & $51(3)$ \\
\hline$C(29)$ & $6358(10)$ & $378(6)$ & $3937(6)$ & $87(4)$ \\
\hline$C(30)$ & $7692(11)$ & $-728(7)$ & $3814(6)$ & $119(5)$ \\
\hline$C(31)$ & $6264(7)$ & $468(5)$ & $681(4)$ & $42(2)$ \\
\hline$C(32)$ & $5580(8)$ & $1221(5)$ & $453(5)$ & $65(3)$ \\
\hline$C(33)$ & $7031(8)$ & $255(6)$ & $143(5)$ & $73(3)$ \\
\hline $0(1)$ & $6530(6)$ & $2905(4)$ & $1690(4)$ & $69(2)$ \\
\hline$c(34)$ & $7560(10)$ & $3226(6)$ & $1502(6)$ & $79(3)$ \\
\hline$C(35)$ & $7213(14)$ & $3500(9)$ & $731(7)$ & $116(5)$ \\
\hline$C(36)$ & $6043(16)$ & $3704(10)$ & $667(9)$ & $146(6)$ \\
\hline$C(37)$ & $5638(10)$ & $3408(7)$ & $1349(6)$ & $88(4)$ \\
\hline
\end{tabular}


Table S21. Bond lengths [A] and angles [deg] for 7.

\begin{tabular}{|c|c|}
\hline $\operatorname{Cr}(1)-N(4)$ & $1.917(7)$ \\
\hline $\operatorname{Cr}(1)-N(2)$ & $1.961(6)$ \\
\hline $\operatorname{Cr}(1)-N(3)$ & $2.017(7)$ \\
\hline $\operatorname{cr}(1)-N(1)$ & $2.0 \odot 8(6)$ \\
\hline $\mathrm{Na}(1)-\mathrm{N}(4)$ & $2.142(8)$ \\
\hline $\mathrm{Na}(1)-0(1)$ & $2.238(7)$ \\
\hline $\mathrm{Na}(1)-\mathrm{C}(6) \# 1$ & $2.878(9)$ \\
\hline $\mathrm{Na}(1)-\mathrm{C}(5) \# 1$ & $2.947(9)$ \\
\hline $\mathrm{Na}(1)-\mathrm{C}(27)$ & $2.926(8)$ \\
\hline $\mathrm{Na}(1)-\mathrm{C}(22)$ & $2.959(8)$ \\
\hline $\mathrm{Na}(1)-\mathrm{C}(26)$ & $2.983(8)$ \\
\hline $\mathrm{Na}(1)-\mathrm{C}(23)$ & $2.996(8)$ \\
\hline $\mathrm{Na}(1)-\mathrm{C}(24)$ & $3.024(9)$ \\
\hline $\mathrm{Na}(1)-\mathrm{C}(25)$ & $3.040(8)$ \\
\hline$N(1)-C(2)$ & $1.377(9)$ \\
\hline$N(1)-C(15)$ & $1.430(9)$ \\
\hline$N(2)-C(3)$ & $1.374(9)$ \\
\hline $\mathrm{N}(2)-\mathrm{C}(7)$ & $1.369(9)$ \\
\hline$N(3)-C(8)$ & $1.375(10)$ \\
\hline$N(3)-C(27)$ & $1.472(10)$ \\
\hline$C(1)-C(2)$ & $1.483(11)$ \\
\hline$C(2)-C(3)$ & $1.403(10)$ \\
\hline$C(3)-C(4)$ & $1.430(10)$ \\
\hline$C(4)-C(5)$ & $1.383(10)$ \\
\hline$C(5)-C(6)$ & $1.398(11)$ \\
\hline
\end{tabular}




\begin{tabular}{|c|c|}
\hline $\mathrm{C}(5)-\mathrm{Na}(1) \# 2$ & $2.947(9)$ \\
\hline$C(6)-C(7)$ & $1.405(10)$ \\
\hline $\mathrm{C}(6)-\mathrm{Na}(1) \# 2$ & $2.878(9)$ \\
\hline$C(7)-C(8)$ & $1.434(10)$ \\
\hline$C(8)-C(9)$ & $1.451(10)$ \\
\hline$C(10)-C(15)$ & $1.388(10)$ \\
\hline$C(10)-C(11)$ & $1.399(11)$ \\
\hline$C(10)-C(16)$ & $1.538(11)$ \\
\hline$C(11)-C(12)$ & $1.369(11)$ \\
\hline$C(12)-C(13)$ & $1.365(12)$ \\
\hline$C(13)-C(14)$ & $1.406(11)$ \\
\hline$C(14)-C(15)$ & $1.412(11)$ \\
\hline$C(14)-C(19)$ & $1.522(11)$ \\
\hline$C(16)-C(18)$ & $1.518(11)$ \\
\hline$C(16)-C(17)$ & $1.530(11)$ \\
\hline$C(19)-C(20)$ & $1.511(13)$ \\
\hline$C(19)-C(21)$ & $1.548(12)$ \\
\hline$C(22)-C(23)$ & $1.387(11)$ \\
\hline$C(22)-C(27)$ & $1.389(10)$ \\
\hline$C(22)-C(28)$ & $1.516(11)$ \\
\hline$C(23)-C(24)$ & $1.366(11)$ \\
\hline$C(24)-C(25)$ & $1.383(11)$ \\
\hline$C(25)-C(26)$ & $1.399(10)$ \\
\hline$C(26)-C(27)$ & $1.397(11)$ \\
\hline$C(26)-C(31)$ & $1.548(11)$ \\
\hline$C(28)-C(29)$ & $1.487(12)$ \\
\hline$C(28)-C(30)$ & $1.529(13)$ \\
\hline$C(31)-C(32)$ & $1.519(11)$ \\
\hline
\end{tabular}




\begin{tabular}{|c|c|}
\hline$c(31)-c(33)$ & $1.509(11)$ \\
\hline $0(1)-C(37)$ & $1.423(11)$ \\
\hline$O(1)-C(34)$ & $1.442(11)$ \\
\hline$c(34)-c(35)$ & $1.493(14)$ \\
\hline$C(35)-C(36)$ & $1.434(17)$ \\
\hline$c(36)-c(37)$ & $1.511(17)$ \\
\hline$N(4)-C r(1)-N(2)$ & $178.5(3)$ \\
\hline$N(4)-C r(1)-N(3)$ & $101.4(3)$ \\
\hline$N(2)-C r(1)-N(3)$ & $77.4(3)$ \\
\hline$N(4)-C r(1)-N(1)$ & $102.9(3)$ \\
\hline$N(2)-C r(1)-N(1)$ & $78.3(3)$ \\
\hline$N(3)-C r(1)-N(1)$ & $155.7(3)$ \\
\hline $\mathrm{N}(4)-\mathrm{Na}(1)-\mathrm{O}(1)$ & $121.0(3)$ \\
\hline$N(4)-N a(1)-C(6) \# 1$ & $96.9(3)$ \\
\hline $0(1)-\mathrm{Na}(1)-\mathrm{C}(6) \# 1$ & $92.6(3)$ \\
\hline $\mathrm{N}(4)-\mathrm{Na}(1)-\mathrm{C}(5) \# 1$ & $120.4(3)$ \\
\hline $\mathrm{O}(1)-\mathrm{Na}(1)-\mathrm{C}(5) \# 1$ & $91.6(3)$ \\
\hline $\mathrm{C}(6) \# 1-\mathrm{Na}(1)-\mathrm{C}(5) \# 1$ & $27.7(2)$ \\
\hline $\mathrm{N}(4)-\mathrm{Na}(1)-\mathrm{C}(27)$ & $77.9(3)$ \\
\hline $\mathrm{O}(1)-\mathrm{Na}(1)-\mathrm{C}(27)$ & $124.6(3)$ \\
\hline $\mathrm{C}(6) \# 1-\mathrm{Na}(1)-\mathrm{C}(27)$ & $139.7(3)$ \\
\hline $\mathrm{C}(5) \# 1-\mathrm{Na}(1)-\mathrm{C}(27)$ & $125.0(3)$ \\
\hline $\mathrm{N}(4)-\mathrm{Na}(1)-\mathrm{C}(22)$ & $89.6(3)$ \\
\hline $\mathrm{O}(1)-\mathrm{Na}(1)-\mathrm{C}(22)$ & $137.0(3)$ \\
\hline $\mathrm{C}(6) \# 1-\mathrm{Na}(1)-\mathrm{C}(22)$ & $114.5(3)$ \\
\hline$C(5) \# 1-\mathrm{Na}(1)-\mathrm{C}(22)$ & $97.8(3)$ \\
\hline$C(27)-\mathrm{Na}(1)-\mathrm{C}(22)$ & $27.3(2)$ \\
\hline$N(4)-N a(1)-C(26)$ & $92.2(3)$ \\
\hline
\end{tabular}




$$
\begin{aligned}
& \text { O(1) }-\mathrm{Na}(1)-\mathrm{C}(26) \quad 97.3(3) \\
& \mathrm{C}(6) \# 1-\mathrm{Na}(1)-\mathrm{C}(26) \quad 160.6(3) \\
& \mathrm{C}(5) \# 1-\mathrm{Na}(1)-\mathrm{C}(26) \quad 134.8(3) \\
& \mathrm{C}(27)-\mathrm{Na}(1)-\mathrm{C}(26) \quad 27.3(2) \\
& \mathrm{C}(22)-\mathrm{Na}(1)-\mathrm{C}(26) \quad 48.3(2) \\
& \mathrm{N}(4)-\mathrm{Na}(1)-\mathrm{C}(23) \quad 116.3(3) \\
& 0(1)-\mathrm{Na}(1)-\mathrm{C}(23) \quad 116.7(3) \\
& \mathrm{C}(6) \# 1-\mathrm{Na}(1)-\mathrm{C}(23) \quad 105.4(3) \\
& \mathrm{C}(5) \# 1-\mathrm{Na}(1)-\mathrm{C}(23) \quad 81.2(2) \\
& \mathrm{C}(27)-\mathrm{Na}(1)-\mathrm{C}(23) \quad 47.4(2) \\
& \mathrm{C}(22)-\mathrm{Na}(1)-\mathrm{C}(23) \quad 26.9(2) \\
& \mathrm{C}(26)-\mathrm{Na}(1)-\mathrm{C}(23) \quad 55.3(2) \\
& \mathrm{N}(4)-\mathrm{Na}(1)-\mathrm{C}(24) \quad 133.2(3) \\
& \mathrm{O}(1)-\mathrm{Na}(1)-\mathrm{C}(24) \quad 91.4(3) \\
& \mathrm{C}(6) \# 1-\mathrm{Na}(1)-\mathrm{C}(24) \quad 115.8(3) \\
& \mathrm{C}(5) \# 1-\mathrm{Na}(1)-\mathrm{C}(24) \quad 88.1(3) \\
& \mathrm{C}(27)-\mathrm{Na}(1)-\mathrm{C}(24) \quad 55.5(2) \\
& \mathrm{C}(22)-\mathrm{Na}(1)-\mathrm{C}(24) \quad 47.5(2) \\
& \mathrm{C}(26)-\mathrm{Na}(1)-\mathrm{C}(24) \quad 47.6(2) \\
& \mathrm{C}(23)-\mathrm{Na}(1)-\mathrm{C}(24) \quad 26.2(2) \\
& \mathrm{N}(4)-\mathrm{Na}(1)-\mathrm{C}(25) \quad 119.0(3) \\
& \mathrm{O}(1)-\mathrm{Na}(1)-\mathrm{C}(25) \quad 82.6(3) \\
& \mathrm{C}(6) \# 1-\mathrm{Na}(1)-\mathrm{C}(25) \quad 140.7(3) \\
& \mathrm{C}(5) \# 1-\mathrm{Na}(1)-\mathrm{C}(25) \quad 113.1(3) \\
& \mathrm{C}(27)-\mathrm{Na}(1)-\mathrm{C}(25) \quad 47.2(2) \\
& \mathrm{C}(22)-\mathrm{Na}(1)-\mathrm{C}(25) \quad 55.1(2) \\
& \mathrm{C}(26)-\mathrm{Na}(1)-\mathrm{C}(25) \quad 26.8(2) \\
& \mathrm{C}(23)-\mathrm{Na}(1)-\mathrm{C}(25) \quad 46.2(2)
\end{aligned}
$$




\begin{tabular}{|c|c|}
\hline $\mathrm{C}(24)-\mathrm{Na}(1)-\mathrm{C}(25)$ & $26.4(2)$ \\
\hline$C(2)-N(1)-C(15)$ & $119.1(6)$ \\
\hline$C(2)-N(1)-C r(1)$ & $115.9(5)$ \\
\hline$C(15)-N(1)-C r(1)$ & $124.8(5)$ \\
\hline$C(3)-N(2)-C(7)$ & $121.9(6)$ \\
\hline$C(3)-N(2)-C r(1)$ & $118.4(5)$ \\
\hline$C(7)-N(2)-C r(1)$ & $119.5(5)$ \\
\hline$C(8)-N(3)-C(27)$ & $119.8(7)$ \\
\hline $\mathrm{C}(8)-\mathrm{N}(3)-\mathrm{Cr}(1)$ & $118.0(6)$ \\
\hline$C(27)-N(3)-C r(1)$ & $122.2(6)$ \\
\hline $\mathrm{Cr}(1)-\mathrm{N}(4)-\mathrm{Na}(1)$ & $129.1(4)$ \\
\hline$N(1)-C(2)-C(3)$ & $114.4(7)$ \\
\hline$N(1)-C(2)-C(1)$ & $122.8(7)$ \\
\hline$C(3)-C(2)-C(1)$ & $122.8(8)$ \\
\hline$N(2)-C(3)-C(2)$ & $112.9(7)$ \\
\hline$N(2)-C(3)-C(4)$ & $118.7(7)$ \\
\hline$c(2)-C(3)-c(4)$ & $128.4(8)$ \\
\hline$c(5)-c(4)-c(3)$ & $119.4(7)$ \\
\hline$C(4)-C(5)-C(6)$ & $120.8(8)$ \\
\hline $\mathrm{C}(4)-\mathrm{C}(5)-\mathrm{Na}(1) \# 2$ & $110.7(6)$ \\
\hline $\mathrm{C}(6)-\mathrm{C}(5)-\mathrm{Na}(1) \# 2$ & $73.4(5)$ \\
\hline$C(5)-C(6)-C(7)$ & $118.9(7)$ \\
\hline $\mathrm{C}(5)-\mathrm{C}(6)-\mathrm{Na}(1) \# 2$ & $78.9(5)$ \\
\hline $\mathrm{C}(7)-\mathrm{C}(6)-\mathrm{Na}(1) \# 2$ & $108.1(5)$ \\
\hline$N(2)-C(7)-C(6)$ & $120.2(7)$ \\
\hline$N(2)-C(7)-C(8)$ & $113.3(7)$ \\
\hline$c(6)-C(7)-C(8)$ & $126.5(8)$ \\
\hline$N(3)-C(8)-C(7)$ & $111.8(7)$ \\
\hline$N(3)-C(8)-C(9)$ & $123.7(7)$ \\
\hline
\end{tabular}




$$
\begin{aligned}
& C(7)-C(8)-C(9) \quad 124.4(7) \\
& C(15)-C(10)-C(11) \quad 118.8(8) \\
& C(15)-C(10)-C(16) \quad 121.7(7) \\
& C(11)-C(10)-C(16) \quad 119.5(8) \\
& C(12)-C(11)-C(10) \quad 122.3(8) \\
& C(13)-C(12)-C(11) \quad 118.7(8) \\
& C(12)-C(13)-C(14) \quad 121.9(8) \\
& C(13)-C(14)-C(15) \quad 118.5(8) \\
& C(13)-C(14)-C(19) \quad 120.2(8) \\
& C(15)-C(14)-C(19) \quad 121.2(7) \\
& C(10)-C(15)-C(14) \quad 119.8(8) \\
& \mathrm{C}(10)-\mathrm{C}(15)-\mathrm{N}(1) \quad 119.9(7) \\
& \mathrm{C}(14)-\mathrm{C}(15)-\mathrm{N}(1) \quad 120.1(7) \\
& C(10)-C(16)-C(18) \quad 111.6(7) \\
& C(10)-C(16)-C(17) \quad 112.2(7) \\
& C(18)-C(16)-C(17) \quad 111.2(7) \\
& C(20)-C(19)-C(14) \quad 112.8(8) \\
& C(20)-C(19)-C(21) \quad 111.3(9) \\
& C(14)-C(19)-C(21) \quad 109.4(7) \\
& C(23)-C(22)-C(27) \quad 118.0(7) \\
& C(23)-C(22)-C(28) \quad 120.6(8) \\
& C(27)-C(22)-C(28) \quad 121.4(8) \\
& \mathrm{C}(23)-\mathrm{C}(22)-\mathrm{Na}(1) \quad 78 . \odot(5) \\
& \mathrm{C}(27)-\mathrm{C}(22)-\mathrm{Na}(1) \quad 75.0(4) \\
& \mathrm{C}(28)-\mathrm{C}(22)-\mathrm{Na}(1) \quad 118.0(5) \\
& C(24)-C(23)-C(22) \quad 122.3(8) \\
& \mathrm{C}(24)-\mathrm{C}(23)-\mathrm{Na}(1) \quad 78.0(5) \\
& \mathrm{C}(22)-\mathrm{C}(23)-\mathrm{Na}(1) \quad 75.0(4)
\end{aligned}
$$




\begin{tabular}{|c|c|}
\hline$C(23)-C(24)-C(25)$ & $118.9(8)$ \\
\hline $\mathrm{C}(23)-\mathrm{C}(24)-\mathrm{Na}(1)$ & $75.7(5)$ \\
\hline$C(25)-C(24)-\mathrm{Na}(1)$ & $77.4(5)$ \\
\hline$C(24)-C(25)-C(26)$ & $121.4(8)$ \\
\hline$C(24)-C(25)-\mathrm{Na}(1)$ & $76.2(5)$ \\
\hline $\mathrm{C}(26)-\mathrm{C}(25)-\mathrm{Na}(1)$ & $74.3(4)$ \\
\hline$C(25)-C(26)-C(27)$ & $117.7(7)$ \\
\hline$C(25)-C(26)-C(31)$ & $119.5(8)$ \\
\hline$C(27)-C(26)-C(31)$ & $122.8(7)$ \\
\hline $\mathrm{C}(25)-\mathrm{C}(26)-\mathrm{Na}(1)$ & $78.8(4)$ \\
\hline$C(27)-C(26)-\mathrm{Na}(1)$ & $74.1(4)$ \\
\hline $\mathrm{C}(31)-\mathrm{C}(26)-\mathrm{Na}(1)$ & $118.5(5)$ \\
\hline$C(26)-C(27)-C(22)$ & $121.6(7)$ \\
\hline$C(26)-C(27)-N(3)$ & $118.2(7)$ \\
\hline$C(22)-C(27)-N(3)$ & $119.8(8)$ \\
\hline$C(26)-C(27)-\mathrm{Na}(1)$ & $78.6(4)$ \\
\hline $\mathrm{C}(22)-\mathrm{C}(27)-\mathrm{Na}(1)$ & $77.7(4)$ \\
\hline$N(3)-C(27)-\mathrm{Na}(1)$ & $109.2(5)$ \\
\hline$C(29)-C(28)-C(22)$ & $112.7(7)$ \\
\hline$C(29)-C(28)-C(30)$ & $110.3(8)$ \\
\hline$C(22)-C(28)-C(30)$ & $113.0(8)$ \\
\hline$C(32)-C(31)-C(33)$ & $111.7(8)$ \\
\hline$C(32)-C(31)-C(26)$ & $110.0(6)$ \\
\hline$C(33)-C(31)-C(26)$ & $113.3(7)$ \\
\hline$c(37)-0(1)-c(34)$ & $106.9(8)$ \\
\hline $\mathrm{C}(37)-0(1)-\mathrm{Na}(1)$ & $120.7(7)$ \\
\hline $\mathrm{C}(34)-0(1)-\mathrm{Na}(1)$ & $132.4(6)$ \\
\hline $0(1)-C(34)-C(35)$ & $103.2(9)$ \\
\hline$C(36)-C(35)-C(34)$ & $105.6(12)$ \\
\hline
\end{tabular}




$$
\begin{array}{ll}
C(35)-C(36)-C(37) & 107.4(12) \\
0(1)-C(37)-C(36) & 103.9(10)
\end{array}
$$

Symmetry transformations used to generate equivalent atoms:

$\# 1-x+1, y+1 / 2,-z+1 / 2 \quad \# 2-x+1, y-1 / 2,-z+1 / 2$ 
Table S22. Anisotropic displacement parameters $\left(A^{\wedge} 2 \times 10 \wedge 3\right)$ for 7. The anisotropic displacement factor exponent takes the form:

$-2 \operatorname{pi} 2\left[h^{\wedge} 2 a^{* \wedge} \mathrm{U} 11+\ldots+2 h k a^{*} b^{*} \mathrm{U} 12\right]$

$\begin{array}{llllll}\text { U11 } & \text { U22 } & \text { U33 } & \text { U23 } & \text { U13 } & \text { U12 }\end{array}$

$\begin{array}{llllrrr}\mathrm{Cr}(1) & 28(1) & 31(1) & 40(1) & -5(1) & 8(1) & 0(1) \\ \mathrm{Na}(1) & 51(2) & 45(2) & 73(3) & -1(2) & 14(2) & -3(2) \\ \mathrm{N}(1) & 35(4) & 18(4) & 39(4) & -8(3) & 13(3) & 2(4) \\ \mathrm{N}(2) & 26(5) & 26(4) & 35(4) & -1(3) & 6(3) & 5(4) \\ \mathrm{N}(3) & 55(4) & 70(4) & 74(4) & -3(3) & 18(3) & 2(3) \\ \mathrm{N}(4) & 55(4) & 70(4) & 74(4) & -3(3) & 18(3) & 2(3) \\ \mathrm{C}(1) & 42(7) & 55(6) & 73(7) & -10(5) & 14(5) & -15(5) \\ \mathrm{C}(2) & 32(6) & 37(6) & 45(6) & 7(5) & 10(4) & 7(5) \\ \mathrm{C}(3) & 35(6) & 31(6) & 29(5) & 0(4) & 3(4) & 2(5) \\ \mathrm{C}(4) & 34(5) & 25(6) & 51(6) & -2(4) & 7(4) & -7(5) \\ \mathrm{C}(5) & 54(7) & 26(5) & 57(7) & -4(4) & 4(5) & -3(5) \\ \mathrm{C}(6) & 36(6) & 37(6) & 43(6) & -4(5) & 6(4) & 11(5) \\ \mathrm{C}(7) & 38(6) & 28(6) & 32(5) & -5(4) & 4(4) & -10(5) \\ \mathrm{C}(8) & 38(6) & 17(5) & 40(5) & -3(4) & 10(4) & 3(4) \\ \mathrm{C}(9) & 46(6) & 47(6) & 54(6) & -10(5) & 13(5) & 7(5) \\ \mathrm{C}(10) & 19(5) & 40(6) & 46(7) & -1(5) & -1(4) & 3(4) \\ \mathrm{C}(11) & 30(6) & 52(7) & 49(7) & -13(6) & 3(5) & 6(5) \\ \mathrm{C}(12) & 38(6) & 42(6) & 73(8) & -20(7) & 16(5) & 7(5) \\ \mathrm{C}(13) & 36(6) & 33(6) & 67(8) & 4(5) & 8(5) & 11(5)\end{array}$




\begin{tabular}{|c|c|c|c|c|c|c|}
\hline$C(14)$ & $32(5)$ & $43(6)$ & $35(6)$ & $-6(5)$ & $8(4)$ & $-6(5)$ \\
\hline$c(15)$ & $21(5)$ & $36(6)$ & $29(6)$ & $-1(5)$ & $8(4)$ & $-16(4)$ \\
\hline$C(16)$ & $33(5)$ & $54(6)$ & $37(6)$ & $\odot(5)$ & $9(5)$ & $\odot(5)$ \\
\hline$c(17)$ & $59(7)$ & $107(9)$ & $68(8)$ & $35(6)$ & $9(6)$ & $-18(6)$ \\
\hline$C(18)$ & $75(8)$ & $92(8)$ & $37(6)$ & $1(6)$ & $1(5)$ & $-7(6)$ \\
\hline$C(19)$ & $54(6)$ & $41(6)$ & $47(7)$ & $16(5)$ & $11(5)$ & $19(5)$ \\
\hline$C(20)$ & $115(11)$ & $170(13)$ & $54(8)$ & $-34(8)$ & $-10(8)$ & $35(9)$ \\
\hline$C(21)$ & $127(10)$ & $86(8)$ & $79(9)$ & $12(7)$ & $59(8)$ & $-5(8)$ \\
\hline$C(22)$ & $33(6)$ & $42(6)$ & $23(6)$ & $4(5)$ & $-2(4)$ & $12(5)$ \\
\hline$c(23)$ & $35(6)$ & $44(6)$ & $39(7)$ & $-11(5)$ & $-7(5)$ & $12(5)$ \\
\hline$C(24)$ & $27(5)$ & $60(7)$ & $55(7)$ & $-1(6)$ & $3(6)$ & $3(5)$ \\
\hline$C(25)$ & $31(6)$ & $51(6)$ & $42(7)$ & $1(5)$ & $21(5)$ & $8(5)$ \\
\hline$C(26)$ & $21(5)$ & $39(5)$ & $45(7)$ & $-6(5)$ & $15(5)$ & $2(4)$ \\
\hline$C(27)$ & $12(5)$ & $29(5)$ & $49(7)$ & $-9(5)$ & $11(5)$ & $\odot(4)$ \\
\hline$C(28)$ & $61(6)$ & $50(6)$ & $36(6)$ & $-7(5)$ & $-10(5)$ & $-10(5)$ \\
\hline$C(29)$ & $119(10)$ & $81(8)$ & $73(8)$ & $11(7)$ & $54(7)$ & $8(7)$ \\
\hline$C(30)$ & $169(13)$ & $104(10)$ & $93(10)$ & $48(8)$ & $49(9)$ & $87(10)$ \\
\hline$c(31)$ & $42(6)$ & $50(6)$ & $35(6)$ & $-9(5)$ & $6(5)$ & $-4(5)$ \\
\hline$C(32)$ & $77(7)$ & $70(7)$ & $43(6)$ & $-7(5)$ & $-5(5)$ & $16(6)$ \\
\hline$C(33)$ & $82(8)$ & $93(8)$ & $52(7)$ & $-10(6)$ & $34(6)$ & $-1(6)$ \\
\hline $0(1)$ & $66(5)$ & $71(5)$ & $73(5)$ & $14(4)$ & $18(4)$ & $7(4)$ \\
\hline$C(34)$ & $120(11)$ & $59(7)$ & $59(8)$ & $\odot(6)$ & $17(7)$ & $-8(7)$ \\
\hline$C(35)$ & $142(14)$ & $126(12)$ & $84(12)$ & $12(9)$ & $29(10)$ & $-50(11)$ \\
\hline$C(36)$ & $171(18)$ & $151(15)$ & $114(15)$ & $39(11)$ & $15(13)$ & $19(13)$ \\
\hline$C(37)$ & $85(9)$ & $111(10)$ & $64(8)$ & $-5(7)$ & $3(7)$ & $45(8)$ \\
\hline
\end{tabular}


Table S23. Hydrogen coordinates $(x 10 \wedge 4)$ and isotropic displacement parameters $\left(A^{\wedge} 2 \times 10^{\wedge} 3\right)$ for 7 .

$\begin{array}{lllll}\text { x } & y & \mathrm{z} & \mathrm{U}(\mathrm{eq})\end{array}$

\begin{tabular}{|c|c|c|c|}
\hline$H(4 B)$ & 4013 & 1718 & 2431 \\
\hline$H(1 A)$ & 193 & 206 & 1927 \\
\hline$H(1 B)$ & 288 & -718 & 2137 \\
\hline$H(1 C)$ & 119 & -455 & 1306 \\
\hline$H(4 A)$ & 1209 & -1855 & 1387 \\
\hline$H(5 A)$ & 2500 & -2789 & 1096 \\
\hline$H(6 A)$ & 4423 & -2479 & 1256 \\
\hline$H(9 A)$ & 7046 & -1180 & 1756 \\
\hline$H(9 B)$ & 6295 & -1703 & 1149 \\
\hline$H(9 C)$ & 6434 & -1963 & 1980 \\
\hline$H(11 A)$ & 1196 & 2313 & 3451 \\
\hline$H(12 A)$ & 473 & 3129 & 2485 \\
\hline$H(13 A)$ & 556 & 2714 & 1312 \\
\hline$H(16 A)$ & 2378 & 342 & 3347 \\
\hline$H(17 A)$ & 562 & 406 & 3661 \\
\hline$H(17 B)$ & 942 & 1141 & 4188 \\
\hline$H(17 C)$ & 1484 & 280 & 4366 \\
\hline$H(18 A)$ & 3713 & 1344 & 3721 \\
\hline$H(18 B)$ & 3423 & 864 & 4404 \\
\hline$H(18 C)$ & 2904 & 1730 & 4220 \\
\hline
\end{tabular}




\begin{tabular}{|c|c|c|c|c|}
\hline$H(19 A)$ & 1715 & 842 & 795 & 57 \\
\hline$H(2 \odot A)$ & -212 & 1091 & 445 & 174 \\
\hline$H(20 B)$ & 445 & 1258 & -214 & 174 \\
\hline$H(20 C)$ & 53 & 1986 & 235 & 174 \\
\hline$H(21 A)$ & 3039 & 1910 & 862 & 139 \\
\hline $\mathrm{H}(21 \mathrm{~B})$ & 2077 & 2499 & 491 & 139 \\
\hline$H(21 C)$ & 2461 & 1768 & 42 & 139 \\
\hline$H(23 A)$ & 8513 & 791 & 3346 & 49 \\
\hline$H(24 A)$ & 9087 & 1476 & 2396 & 57 \\
\hline$H(25 A)$ & 8148 & 1267 & 1214 & 48 \\
\hline$H(28 A)$ & 6134 & -528 & 3210 & 61 \\
\hline$H(29 A)$ & 5765 & 714 & 3678 & 130 \\
\hline$H(29 B)$ & 6973 & 714 & 4169 & 130 \\
\hline$H(29 C)$ & 6063 & 67 & 4307 & 130 \\
\hline$H(3 \odot A)$ & 7412 & -996 & 4213 & 178 \\
\hline$H(3 \odot B)$ & 8351 & -410 & 4006 & 178 \\
\hline$H(3 \odot C)$ & 7896 & -1127 & 3477 & 178 \\
\hline$H(31 A)$ & 5722 & 19 & 681 & 51 \\
\hline$H(32 A)$ & 5094 & 1338 & 808 & 97 \\
\hline$H(32 B)$ & 5124 & 1136 & -23 & 97 \\
\hline$H(32 C)$ & 6087 & 1671 & 427 & 97 \\
\hline$H(33 A)$ & 6589 & 201 & -341 & 110 \\
\hline$H(33 B)$ & 7409 & -250 & 285 & 110 \\
\hline$H(33 C)$ & 7588 & 677 & 139 & 110 \\
\hline$H(34 A)$ & 8145 & 2811 & 1535 & 95 \\
\hline $\mathrm{H}(34 \mathrm{~B})$ & 7841 & 3677 & 1821 & 95 \\
\hline$H(35 A)$ & 7656 & 3969 & 629 & 140 \\
\hline$H(35 B)$ & 7322 & 3069 & 389 & 140 \\
\hline
\end{tabular}




\begin{tabular}{lrrrr}
$H(36 A)$ & 5611 & 3448 & 234 & 175 \\
$H(36 B)$ & 5943 & 4288 & 620 & 175 \\
$H(37 A)$ & 5515 & 3858 & 1668 & 105 \\
$H(37 B)$ & 4935 & 3103 & 1227 & 105 \\
\hline
\end{tabular}


Table S24. Torsion angles [deg] for $\mathbf{7}$.

$$
\begin{aligned}
& N(4)-\operatorname{Cr}(1)-N(1)-C(2) \\
& 177.2(5) \\
& \mathrm{N}(2)-\mathrm{Cr}(1)-\mathrm{N}(1)-\mathrm{C}(2) \\
& -1.8(5) \\
& N(3)-\operatorname{Cr}(1)-N(1)-C(2) \\
& -1.1(10) \\
& N(4)-\operatorname{Cr}(1)-N(1)-C(15) \\
& 3.6(6) \\
& \mathrm{N}(2)-\mathrm{Cr}(1)-\mathrm{N}(1)-\mathrm{C}(15) \\
& -175.4(6) \\
& N(3)-\operatorname{Cr}(1)-N(1)-C(15) \\
& -174.7(7) \\
& \mathrm{N}(4)-\mathrm{Cr}(1)-\mathrm{N}(2)-\mathrm{C}(3) \\
& -139(12) \\
& \mathrm{N}(3)-\mathrm{Cr}(1)-\mathrm{N}(2)-\mathrm{C}(3) \\
& -177.2(6) \\
& N(1)-C r(1)-N(2)-C(3) \\
& 2.5(5) \\
& \mathrm{N}(4)-\mathrm{Cr}(1)-\mathrm{N}(2)-\mathrm{C}(7) \\
& 37 \text { (12) } \\
& \mathrm{N}(3)-\mathrm{Cr}(1)-\mathrm{N}(2)-\mathrm{C}(7) \\
& -1.7(6) \\
& \mathrm{N}(1)-\mathrm{Cr}(1)-\mathrm{N}(2)-\mathrm{C}(7) \\
& 178 . \odot(6) \\
& \mathrm{N}(4)-\mathrm{Cr}(1)-\mathrm{N}(3)-\mathrm{C}(8) \\
& -177.9(6) \\
& N(2)-C r(1)-N(3)-C(8) \\
& 1.1(6) \\
& \mathrm{N}(1)-\mathrm{Cr}(1)-\mathrm{N}(3)-\mathrm{C}(8) \\
& 0.4 \text { (11) } \\
& \mathrm{N}(4)-\mathrm{Cr}(1)-\mathrm{N}(3)-\mathrm{C}(27) \\
& 0.2(7) \\
& N(2)-\operatorname{Cr}(1)-N(3)-C(27) \\
& 179.3(7) \\
& \mathrm{N}(1)-\mathrm{Cr}(1)-\mathrm{N}(3)-\mathrm{C}(27) \\
& 178.5(6) \\
& \mathrm{N}(2)-\mathrm{Cr}(1)-\mathrm{N}(4)-\mathrm{Na}(1) \\
& -34(12) \\
& \mathrm{N}(3)-\mathrm{Cr}(1)-\mathrm{N}(4)-\mathrm{Na}(1) \\
& \text { 4. 4(5) } \\
& \mathrm{N}(1)-\mathrm{Cr}(1)-\mathrm{N}(4)-\mathrm{Na}(1) \\
& -174.9(4) \\
& \mathrm{O}(1)-\mathrm{Na}(1)-\mathrm{N}(4)-\mathrm{Cr}(1) \\
& 118.6(5) \\
& \mathrm{C}(6) \# 1-\mathrm{Na}(1)-\mathrm{N}(4)-\mathrm{Cr}(1) \\
& -144.1(5) \\
& \mathrm{C}(5) \# 1-\mathrm{Na}(1)-\mathrm{N}(4)-\mathrm{Cr}(1) \\
& -128.4(4) \\
& \mathrm{C}(27)-\mathrm{Na}(1)-\mathrm{N}(4)-\mathrm{Cr}(1) \\
& -4.7(5)
\end{aligned}
$$




$$
\begin{aligned}
& \mathrm{C}(22)-\mathrm{Na}(1)-\mathrm{N}(4)-\mathrm{Cr}(1) \\
& -29.5(5) \\
& \mathrm{C}(26)-\mathrm{Na}(1)-\mathrm{N}(4)-\mathrm{Cr}(1) \\
& \text { 18.7(5) } \\
& \mathrm{C}(23)-\mathrm{Na}(1)-\mathrm{N}(4)-\mathrm{Cr}(1) \\
& -33.2(6) \\
& \mathrm{C}(24)-\mathrm{Na}(1)-\mathrm{N}(4)-\mathrm{Cr}(1) \\
& -8.6(7) \\
& \mathrm{C}(25)-\mathrm{Na}(1)-\mathrm{N}(4)-\mathrm{Cr}(1) \\
& 19.3(6) \\
& C(15)-N(1)-C(2)-C(3) \\
& \mathrm{Cr}(1)-\mathrm{N}(1)-\mathrm{C}(2)-\mathrm{C}(3) \\
& C(15)-N(1)-C(2)-C(1) \\
& \mathrm{Cr}(1)-\mathrm{N}(1)-\mathrm{C}(2)-\mathrm{C}(1) \\
& C(7)-N(2)-C(3)-C(2) \\
& \mathrm{Cr}(1)-\mathrm{N}(2)-\mathrm{C}(3)-\mathrm{C}(2) \\
& C(7)-N(2)-C(3)-C(4) \\
& \operatorname{Cr}(1)-N(2)-C(3)-C(4) \\
& \mathrm{N}(1)-\mathrm{C}(2)-\mathrm{C}(3)-\mathrm{N}(2) \\
& C(1)-C(2)-C(3)-N(2) \\
& N(1)-C(2)-C(3)-C(4) \\
& C(1)-C(2)-C(3)-C(4) \\
& N(2)-C(3)-C(4)-C(5) \\
& C(2)-C(3)-C(4)-C(5) \\
& C(3)-C(4)-C(5)-C(6) \\
& \mathrm{C}(3)-\mathrm{C}(4)-\mathrm{C}(5)-\mathrm{Na}(1) \# 2 \\
& C(4)-C(5)-C(6)-C(7) \\
& \mathrm{Na}(1) \# 2-\mathrm{C}(5)-\mathrm{C}(6)-\mathrm{C}(7) \\
& C(4)-C(5)-C(6)-\mathrm{Na}(1) \# 2 \\
& \mathrm{C}(3)-\mathrm{N}(2)-\mathrm{C}(7)-\mathrm{C}(6) \\
& \operatorname{Cr}(1)-N(2)-C(7)-C(6) \\
& \mathrm{C}(3)-\mathrm{N}(2)-\mathrm{C}(7)-\mathrm{C}(8) \\
& \operatorname{Cr}(1)-N(2)-C(7)-C(8) \\
& 175 . \odot(6) \\
& 1.0(8) \\
& -6.5(11) \\
& \text { 179.5(6) } \\
& -178 \cdot 1(7) \\
& -2.7(8) \\
& 1.8(10) \\
& \text { 177.2(5) } \\
& \text { 1. } \odot(9) \\
& -177.5(7) \\
& -178.8(7) \\
& 2.7(13) \\
& -3.1(11) \\
& 176.7(8) \\
& 2.3(12) \\
& 84.8(8) \\
& -0.1(12) \\
& -104.7(7) \\
& \text { 104.5(8) } \\
& 0.4(11) \\
& -174.9(6) \\
& 177.3(7) \\
& \text { 1.9(9) }
\end{aligned}
$$




$$
\begin{aligned}
& C(5)-C(6)-C(7)-N(2) \\
& -1.3(11) \\
& \mathrm{Na}(1) \# 2-\mathrm{C}(6)-\mathrm{C}(7)-\mathrm{N}(2) \\
& -88.3(7) \\
& C(5)-C(6)-C(7)-C(8) \\
& -177.7(8) \\
& \mathrm{Na}(1) \# 2-\mathrm{C}(6)-\mathrm{C}(7)-\mathrm{C}(8) \\
& 95.3(8) \\
& C(27)-N(3)-C(8)-C(7) \\
& -178.7(7) \\
& \mathrm{Cr}(1)-\mathrm{N}(3)-\mathrm{C}(8)-\mathrm{C}(7) \\
& -0.5(9) \\
& C(27)-N(3)-C(8)-C(9) \\
& \text { 3. 2(12) } \\
& \operatorname{Cr}(1)-\mathrm{N}(3)-\mathrm{C}(8)-\mathrm{C}(9) \\
& -178.6(6) \\
& \mathrm{N}(2)-\mathrm{C}(7)-\mathrm{C}(8)-\mathrm{N}(3) \\
& -\odot .8(10) \\
& \mathrm{C}(6)-\mathrm{C}(7)-\mathrm{C}(8)-\mathrm{N}(3) \\
& 175.7(8) \\
& \mathrm{N}(2)-\mathrm{C}(7)-\mathrm{C}(8)-\mathrm{C}(9) \\
& 177.3(7) \\
& C(6)-C(7)-C(8)-C(9) \\
& -6.1(13) \\
& C(15)-C(10)-C(11)-C(12) \\
& \text { 1.6(12) } \\
& C(16)-C(10)-C(11)-C(12) \\
& C(10)-C(11)-C(12)-C(13) \\
& C(11)-C(12)-C(13)-C(14) \\
& C(12)-C(13)-C(14)-C(15) \\
& C(12)-C(13)-C(14)-C(19) \\
& C(11)-C(10)-C(15)-C(14) \\
& C(16)-C(10)-C(15)-C(14) \\
& \mathrm{C}(11)-\mathrm{C}(10)-\mathrm{C}(15)-\mathrm{N}(1) \\
& \mathrm{C}(16)-\mathrm{C}(10)-\mathrm{C}(15)-\mathrm{N}(1) \\
& C(13)-C(14)-C(15)-C(10) \\
& C(19)-C(14)-C(15)-C(10) \\
& \mathrm{C}(13)-\mathrm{C}(14)-\mathrm{C}(15)-\mathrm{N}(1) \\
& C(19)-C(14)-C(15)-N(1) \\
& C(2)-N(1)-C(15)-C(10) \\
& \mathrm{Cr}(1)-\mathrm{N}(1)-\mathrm{C}(15)-\mathrm{C}(10) \\
& C(2)-N(1)-C(15)-C(14) \\
& -178.5(7) \\
& -1.1(12) \\
& -0.1(12) \\
& 0.6(12) \\
& -175.7(8) \\
& -1.1(11) \\
& \text { 179.1(7) } \\
& 173.4(6) \\
& -6.4(10) \\
& \odot . \odot(11) \\
& 176.3(7) \\
& -174.5(6) \\
& 1.8(11) \\
& 102.3(8) \\
& -84.3(8) \\
& -83.3(8)
\end{aligned}
$$




$$
\begin{aligned}
& \operatorname{Cr}(1)-N(1)-C(15)-C(14) \quad 90.2(8) \\
& C(15)-C(10)-C(16)-C(18) \quad 114.0(9) \\
& C(11)-C(10)-C(16)-C(18) \quad-65.9(9) \\
& C(15)-C(10)-C(16)-C(17) \quad-120.5(8) \\
& C(11)-C(10)-C(16)-C(17) \quad 59.6(10) \\
& C(13)-C(14)-C(19)-C(20) \quad-55.4(11) \\
& C(15)-C(14)-C(19)-C(20) \quad 128.3(9) \\
& C(13)-C(14)-C(19)-C(21) \quad 69.0(10) \\
& C(15)-C(14)-C(19)-C(21) \quad-107.2(9) \\
& \mathrm{N}(4)-\mathrm{Na}(1)-\mathrm{C}(22)-\mathrm{C}(23) \quad-172.7(5) \\
& \mathrm{O}(1)-\mathrm{Na}(1)-\mathrm{C}(22)-\mathrm{C}(23) \quad 48.8(7) \\
& \mathrm{C}(6) \# 1-\mathrm{Na}(1)-\mathrm{C}(22)-\mathrm{C}(23) \quad-75.4(5) \\
& \mathrm{C}(5) \# 1-\mathrm{Na}(1)-\mathrm{C}(22)-\mathrm{C}(23) \quad-52.1(5) \\
& \mathrm{C}(27)-\mathrm{Na}(1)-\mathrm{C}(22)-\mathrm{C}(23) \quad 123.6(7) \\
& \mathrm{C}(26)-\mathrm{Na}(1)-\mathrm{C}(22)-\mathrm{C}(23) \quad 93.9(5) \\
& \mathrm{C}(24)-\mathrm{Na}(1)-\mathrm{C}(22)-\mathrm{C}(23) \quad 28.0(5) \\
& \mathrm{C}(25)-\mathrm{Na}(1)-\mathrm{C}(22)-\mathrm{C}(23) \quad 60.6(5) \\
& \mathrm{N}(4)-\mathrm{Na}(1)-\mathrm{C}(22)-\mathrm{C}(27) \quad 63.7(5) \\
& \mathrm{O}(1)-\mathrm{Na}(1)-\mathrm{C}(22)-\mathrm{C}(27) \quad-74.8(6) \\
& \mathrm{C}(6) \# 1-\mathrm{Na}(1)-\mathrm{C}(22)-\mathrm{C}(27) \quad 161.0(5) \\
& \mathrm{C}(5) \# 1-\mathrm{Na}(1)-\mathrm{C}(22)-\mathrm{C}(27) \quad-175.7(5) \\
& \mathrm{C}(26)-\mathrm{Na}(1)-\mathrm{C}(22)-\mathrm{C}(27) \quad-29.7(4) \\
& \mathrm{C}(23)-\mathrm{Na}(1)-\mathrm{C}(22)-\mathrm{C}(27) \quad-123.6(7) \\
& \mathrm{C}(24)-\mathrm{Na}(1)-\mathrm{C}(22)-\mathrm{C}(27) \quad-95.6(5) \\
& \mathrm{C}(25)-\mathrm{Na}(1)-\mathrm{C}(22)-\mathrm{C}(27) \quad-63.0(5) \\
& \mathrm{N}(4)-\mathrm{Na}(1)-\mathrm{C}(22)-\mathrm{C}(28) \quad-54.3(6) \\
& \mathrm{O}(1)-\mathrm{Na}(1)-\mathrm{C}(22)-\mathrm{C}(28) \quad 167.3(6) \\
& \mathrm{C}(6) \# 1-\mathrm{Na}(1)-\mathrm{C}(22)-\mathrm{C}(28) \quad 43.1(7)
\end{aligned}
$$




$$
\begin{aligned}
& \mathrm{C}(5) \# 1-\mathrm{Na}(1)-\mathrm{C}(22)-\mathrm{C}(28) \\
& 66.3(6) \\
& \mathrm{C}(27)-\mathrm{Na}(1)-\mathrm{C}(22)-\mathrm{C}(28) \\
& -117.9(9) \\
& \mathrm{C}(26)-\mathrm{Na}(1)-\mathrm{C}(22)-\mathrm{C}(28) \\
& -147.6(8) \\
& \mathrm{C}(23)-\mathrm{Na}(1)-\mathrm{C}(22)-\mathrm{C}(28) \\
& 118.5(9) \\
& \mathrm{C}(24)-\mathrm{Na}(1)-\mathrm{C}(22)-\mathrm{C}(28) \\
& 146.5(8) \\
& \mathrm{C}(25)-\mathrm{Na}(1)-\mathrm{C}(22)-\mathrm{C}(28) \\
& \text { 179.1(8) } \\
& C(27)-C(22)-C(23)-C(24) \\
& 0.7(12) \\
& C(28)-C(22)-C(23)-C(24) \\
& 179.4(8) \\
& \mathrm{Na}(1)-\mathrm{C}(22)-\mathrm{C}(23)-\mathrm{C}(24) \\
& -65 . \odot(7) \\
& \mathrm{C}(27)-\mathrm{C}(22)-\mathrm{C}(23)-\mathrm{Na}(1) \\
& 65.7(6) \\
& C(28)-C(22)-C(23)-\mathrm{Na}(1) \\
& -115 \cdot 6(7) \\
& \mathrm{N}(4)-\mathrm{Na}(1)-\mathrm{C}(23)-\mathrm{C}(24) \\
& 136.6(6) \\
& O(1)-\mathrm{Na}(1)-\mathrm{C}(23)-\mathrm{C}(24) \\
& -16.5(6) \\
& \mathrm{C}(6) \# 1-\mathrm{Na}(1)-\mathrm{C}(23)-\mathrm{C}(24) \\
& -117.5(6) \\
& \mathrm{C}(5) \# 1-\mathrm{Na}(1)-\mathrm{C}(23)-\mathrm{C}(24) \\
& -103.9(6) \\
& \mathrm{C}(27)-\mathrm{Na}(1)-\mathrm{C}(23)-\mathrm{C}(24) \\
& 97.2(6) \\
& \mathrm{C}(22)-\mathrm{Na}(1)-\mathrm{C}(23)-\mathrm{C}(24) \\
& 128.4(8) \\
& \mathrm{C}(26)-\mathrm{Na}(1)-\mathrm{C}(23)-\mathrm{C}(24) \\
& 63.4(5) \\
& \mathrm{C}(25)-\mathrm{Na}(1)-\mathrm{C}(23)-\mathrm{C}(24) \\
& 30.5(5) \\
& \mathrm{N}(4)-\mathrm{Na}(1)-\mathrm{C}(23)-\mathrm{C}(22) \\
& \text { 8.1(6) } \\
& \mathrm{O}(1)-\mathrm{Na}(1)-\mathrm{C}(23)-\mathrm{C}(22) \\
& \mathrm{C}(6) \# 1-\mathrm{Na}(1)-\mathrm{C}(23)-\mathrm{C}(22) \\
& -144.9(5) \\
& 114.0(5) \\
& \mathrm{C}(5) \# 1-\mathrm{Na}(1)-\mathrm{C}(23)-\mathrm{C}(22) \\
& 127.7(5) \\
& \mathrm{C}(27)-\mathrm{Na}(1)-\mathrm{C}(23)-\mathrm{C}(22) \\
& -31.3(4) \\
& \mathrm{C}(26)-\mathrm{Na}(1)-\mathrm{C}(23)-\mathrm{C}(22) \\
& -65.1(5) \\
& \mathrm{C}(24)-\mathrm{Na}(1)-\mathrm{C}(23)-\mathrm{C}(22) \\
& -128 \cdot 4(8) \\
& \mathrm{C}(25)-\mathrm{Na}(1)-\mathrm{C}(23)-\mathrm{C}(22) \\
& -98 \cdot \odot(5) \\
& C(22)-C(23)-C(24)-C(25) \\
& -3.2(12) \\
& \mathrm{Na}(1)-\mathrm{C}(23)-\mathrm{C}(24)-\mathrm{C}(25) \\
& -66.7(7)
\end{aligned}
$$




$$
\begin{aligned}
& \mathrm{C}(22)-\mathrm{C}(23)-\mathrm{C}(24)-\mathrm{Na}(1) \quad 63.5(7) \\
& \mathrm{N}(4)-\mathrm{Na}(1)-\mathrm{C}(24)-\mathrm{C}(23) \quad-57.8(7) \\
& \mathrm{O}(1)-\mathrm{Na}(1)-\mathrm{C}(24)-\mathrm{C}(23) \quad 165.3(6) \\
& \mathrm{C}(6) \# 1-\mathrm{Na}(1)-\mathrm{C}(24)-\mathrm{C}(23) \quad 71.7(6) \\
& \mathrm{C}(5) \# 1-\mathrm{Na}(1)-\mathrm{C}(24)-\mathrm{C}(23) \quad 73.7(6) \\
& \mathrm{C}(27)-\mathrm{Na}(1)-\mathrm{C}(24)-\mathrm{C}(23) \quad-62.4(5) \\
& \mathrm{C}(22)-\mathrm{Na}(1)-\mathrm{C}(24)-\mathrm{C}(23) \quad-28.8(5) \\
& \mathrm{C}(26)-\mathrm{Na}(1)-\mathrm{C}(24)-\mathrm{C}(23) \quad-96.1(6) \\
& \mathrm{C}(25)-\mathrm{Na}(1)-\mathrm{C}(24)-\mathrm{C}(23) \quad-124.5(8) \\
& \mathrm{N}(4)-\mathrm{Na}(1)-\mathrm{C}(24)-\mathrm{C}(25) \quad 66.8(7) \\
& \mathrm{O}(1)-\mathrm{Na}(1)-\mathrm{C}(24)-\mathrm{C}(25) \quad-70.2(6) \\
& \mathrm{C}(6) \# 1-\mathrm{Na}(1)-\mathrm{C}(24)-\mathrm{C}(25) \quad-163.8(5) \\
& \mathrm{C}(5) \# 1-\mathrm{Na}(1)-\mathrm{C}(24)-\mathrm{C}(25) \quad-161.8(6) \\
& \mathrm{C}(27)-\mathrm{Na}(1)-\mathrm{C}(24)-\mathrm{C}(25) \quad 62.1(5) \\
& \mathrm{C}(22)-\mathrm{Na}(1)-\mathrm{C}(24)-\mathrm{C}(25) \quad 95.8(6) \\
& \mathrm{C}(26)-\mathrm{Na}(1)-\mathrm{C}(24)-\mathrm{C}(25) \quad 28.4(5) \\
& \mathrm{C}(23)-\mathrm{Na}(1)-\mathrm{C}(24)-\mathrm{C}(25) \quad 124.5(8) \\
& C(23)-C(24)-C(25)-C(26) \quad 4.2(12) \\
& \mathrm{Na}(1)-\mathrm{C}(24)-\mathrm{C}(25)-\mathrm{C}(26) \quad-61.5(7) \\
& \mathrm{C}(23)-\mathrm{C}(24)-\mathrm{C}(25)-\mathrm{Na}(1) \quad 65.8(7) \\
& \mathrm{N}(4)-\mathrm{Na}(1)-\mathrm{C}(25)-\mathrm{C}(24) \quad-130.0(6) \\
& \mathrm{O}(1)-\mathrm{Na}(1)-\mathrm{C}(25)-\mathrm{C}(24) \quad 108.5(6) \\
& \begin{array}{ll}
\mathrm{C}(6) \# 1-\mathrm{Na}(1)-\mathrm{C}(25)-\mathrm{C}(24) & 23.4(7)
\end{array} \\
& \mathrm{C}(5) \# 1-\mathrm{Na}(1)-\mathrm{C}(25)-\mathrm{C}(24) \quad 19.9(6) \\
& \mathrm{C}(27)-\mathrm{Na}(1)-\mathrm{C}(25)-\mathrm{C}(24) \quad-97.3(6) \\
& \mathrm{C}(22)-\mathrm{Na}(1)-\mathrm{C}(25)-\mathrm{C}(24) \quad-63.5(5) \\
& \mathrm{C}(26)-\mathrm{Na}(1)-\mathrm{C}(25)-\mathrm{C}(24) \quad-128.8(8) \\
& \mathrm{C}(23)-\mathrm{Na}(1)-\mathrm{C}(25)-\mathrm{C}(24) \quad-30.3(5)
\end{aligned}
$$




$$
\begin{aligned}
& \mathrm{N}(4)-\mathrm{Na}(1)-\mathrm{C}(25)-\mathrm{C}(26) \\
& -1.2(6) \\
& \mathrm{O}(1)-\mathrm{Na}(1)-\mathrm{C}(25)-\mathrm{C}(26) \\
& -122.7(5) \\
& \mathrm{C}(6) \# 1-\mathrm{Na}(1)-\mathrm{C}(25)-\mathrm{C}(26) \\
& 152.2(5) \\
& \mathrm{C}(5) \# 1-\mathrm{Na}(1)-\mathrm{C}(25)-\mathrm{C}(26) \\
& 148.7(5) \\
& \mathrm{C}(27)-\mathrm{Na}(1)-\mathrm{C}(25)-\mathrm{C}(26) \\
& 31.5(4) \\
& \mathrm{C}(22)-\mathrm{Na}(1)-\mathrm{C}(25)-\mathrm{C}(26) \\
& 65.4(5) \\
& \mathrm{C}(23)-\mathrm{Na}(1)-\mathrm{C}(25)-\mathrm{C}(26) \\
& 98.5(6) \\
& \mathrm{C}(24)-\mathrm{Na}(1)-\mathrm{C}(25)-\mathrm{C}(26) \\
& 128.8(8) \\
& C(24)-C(25)-C(26)-C(27) \\
& -2.8(11) \\
& \mathrm{Na}(1)-\mathrm{C}(25)-\mathrm{C}(26)-\mathrm{C}(27) \\
& -65.2(6) \\
& C(24)-C(25)-C(26)-C(31) \\
& 179 \cdot \odot(7) \\
& \mathrm{Na}(1)-\mathrm{C}(25)-\mathrm{C}(26)-\mathrm{C}(31) \\
& 116.5(6) \\
& C(24)-C(25)-C(26)-\mathrm{Na}(1) \\
& 62.5(7) \\
& \mathrm{N}(4)-\mathrm{Na}(1)-\mathrm{C}(26)-\mathrm{C}(25) \\
& \mathrm{O}(1)-\mathrm{Na}(1)-\mathrm{C}(26)-\mathrm{C}(25) \\
& 179 . \odot(5) \\
& 57.3(5) \\
& \mathrm{C}(6) \# 1-\mathrm{Na}(1)-\mathrm{C}(26)-\mathrm{C}(25) \\
& -62.9(10) \\
& \mathrm{C}(5) \# 1-\mathrm{Na}(1)-\mathrm{C}(26)-\mathrm{C}(25) \\
& -42.3(6) \\
& \mathrm{C}(27)-\mathrm{Na}(1)-\mathrm{C}(26)-\mathrm{C}(25) \\
& -123.3(7) \\
& C(22)-\mathrm{Na}(1)-\mathrm{C}(26)-\mathrm{C}(25) \\
& -93.6(5) \\
& \mathrm{C}(23)-\mathrm{Na}(1)-\mathrm{C}(26)-\mathrm{C}(25) \\
& -60.2(5) \\
& \mathrm{C}(24)-\mathrm{Na}(1)-\mathrm{C}(26)-\mathrm{C}(25) \\
& -27.9(5) \\
& \mathrm{N}(4)-\mathrm{Na}(1)-\mathrm{C}(26)-\mathrm{C}(27) \\
& O(1)-\mathrm{Na}(1)-\mathrm{C}(26)-\mathrm{C}(27) \\
& -57.8(5) \\
& -179.4(5) \\
& \mathrm{C}(6) \# 1-\mathrm{Na}(1)-\mathrm{C}(26)-\mathrm{C}(27) \\
& 60.4(10) \\
& \mathrm{C}(5) \# 1-\mathrm{Na}(1)-\mathrm{C}(26)-\mathrm{C}(27) \\
& 80.9(6) \\
& \mathrm{C}(22)-\mathrm{Na}(1)-\mathrm{C}(26)-\mathrm{C}(27) \\
& 29.7(4) \\
& \mathrm{C}(23)-\mathrm{Na}(1)-\mathrm{C}(26)-\mathrm{C}(27) \\
& 63 . \odot(5) \\
& \mathrm{C}(24)-\mathrm{Na}(1)-\mathrm{C}(26)-\mathrm{C}(27) \\
& 95.3(5) \\
& \mathrm{C}(25)-\mathrm{Na}(1)-\mathrm{C}(26)-\mathrm{C}(27) \\
& 123.3(7)
\end{aligned}
$$




$$
\begin{aligned}
& \mathrm{N}(4)-\mathrm{Na}(1)-\mathrm{C}(26)-\mathrm{C}(31) \quad 61.4(6) \\
& \mathrm{O}(1)-\mathrm{Na}(1)-\mathrm{C}(26)-\mathrm{C}(31) \quad-60.3(6) \\
& \mathrm{C}(6) \# 1-\mathrm{Na}(1)-\mathrm{C}(26)-\mathrm{C}(31) \quad 179.5(7) \\
& \mathrm{C}(5) \# 1-\mathrm{Na}(1)-\mathrm{C}(26)-\mathrm{C}(31) \quad-160 . \odot(6) \\
& \mathrm{C}(27)-\mathrm{Na}(1)-\mathrm{C}(26)-\mathrm{C}(31) \quad 119.1(8) \\
& \mathrm{C}(22)-\mathrm{Na}(1)-\mathrm{C}(26)-\mathrm{C}(31) \quad 148.8(7) \\
& \mathrm{C}(23)-\mathrm{Na}(1)-\mathrm{C}(26)-\mathrm{C}(31) \quad-177.8(7) \\
& \mathrm{C}(24)-\mathrm{Na}(1)-\mathrm{C}(26)-\mathrm{C}(31) \quad-145.5(8) \\
& \mathrm{C}(25)-\mathrm{Na}(1)-\mathrm{C}(26)-\mathrm{C}(31) \quad-117.6(9) \\
& C(25)-C(26)-C(27)-C(22) \quad 0.2(11) \\
& \mathrm{C}(31)-\mathrm{C}(26)-\mathrm{C}(27)-\mathrm{C}(22) \quad 178.4(7) \\
& \mathrm{Na}(1)-\mathrm{C}(26)-\mathrm{C}(27)-\mathrm{C}(22) \quad-67.6(7) \\
& \begin{array}{ll}
C(25)-C(26)-C(27)-N(3) & 173.7(7)
\end{array} \\
& \mathrm{C}(31)-\mathrm{C}(26)-\mathrm{C}(27)-\mathrm{N}(3) \quad-8.1(11) \\
& \mathrm{Na}(1)-\mathrm{C}(26)-\mathrm{C}(27)-\mathrm{N}(3) \quad 105.8(6) \\
& \mathrm{C}(25)-\mathrm{C}(26)-\mathrm{C}(27)-\mathrm{Na}(1) \quad 67.9(6) \\
& \mathrm{C}(31)-\mathrm{C}(26)-\mathrm{C}(27)-\mathrm{Na}(1) \quad-113.9(7) \\
& C(23)-C(22)-C(27)-C(26) \quad 0.8(11) \\
& C(28)-C(22)-C(27)-C(26) \quad-177.9(7) \\
& \mathrm{Na}(1)-\mathrm{C}(22)-\mathrm{C}(27)-\mathrm{C}(26) \quad 68.1(7) \\
& \mathrm{C}(23)-\mathrm{C}(22)-\mathrm{C}(27)-\mathrm{N}(3) \quad-172.6(7) \\
& \mathrm{C}(28)-\mathrm{C}(22)-\mathrm{C}(27)-\mathrm{N}(3) \quad 8.7(11) \\
& \mathrm{Na}(1)-\mathrm{C}(22)-\mathrm{C}(27)-\mathrm{N}(3) \quad-105.3(6) \\
& \begin{array}{ll}
\mathrm{C}(23)-\mathrm{C}(22)-\mathrm{C}(27)-\mathrm{Na}(1) & -67.3(6)
\end{array} \\
& \begin{array}{ll}
\mathrm{C}(28)-\mathrm{C}(22)-\mathrm{C}(27)-\mathrm{Na}(1) & 114.0(7)
\end{array} \\
& \mathrm{C}(8)-\mathrm{N}(3)-\mathrm{C}(27)-\mathrm{C}(26) \quad 88.1(9) \\
& \mathrm{Cr}(1)-\mathrm{N}(3)-\mathrm{C}(27)-\mathrm{C}(26) \quad-90 . \odot(8) \\
& \mathrm{C}(8)-\mathrm{N}(3)-\mathrm{C}(27)-\mathrm{C}(22) \quad-98.3(9)
\end{aligned}
$$




$$
\begin{aligned}
& \mathrm{Cr}(1)-\mathrm{N}(3)-\mathrm{C}(27)-\mathrm{C}(22) \\
& 83.6(8) \\
& \mathrm{C}(8)-\mathrm{N}(3)-\mathrm{C}(27)-\mathrm{Na}(1) \\
& 175.2(6) \\
& \mathrm{Cr}(1)-\mathrm{N}(3)-\mathrm{C}(27)-\mathrm{Na}(1) \\
& -2.9(8) \\
& \mathrm{N}(4)-\mathrm{Na}(1)-\mathrm{C}(27)-\mathrm{C}(26) \\
& 120.2(5) \\
& O(1)-\mathrm{Na}(1)-\mathrm{C}(27)-\mathrm{C}(26) \\
& \odot .7(6) \\
& \mathrm{C}(6) \# 1-\mathrm{Na}(1)-\mathrm{C}(27)-\mathrm{C}(26) \\
& -153.5(5) \\
& \mathrm{C}(5) \# 1-\mathrm{Na}(1)-\mathrm{C}(27)-\mathrm{C}(26) \\
& -121.1(5) \\
& \mathrm{C}(22)-\mathrm{Na}(1)-\mathrm{C}(27)-\mathrm{C}(26) \\
& -126 \cdot 3(7) \\
& \mathrm{C}(23)-\mathrm{Na}(1)-\mathrm{C}(27)-\mathrm{C}(26) \\
& -95.4(5) \\
& \mathrm{C}(24)-\mathrm{Na}(1)-\mathrm{C}(27)-\mathrm{C}(26) \\
& -63 \cdot 3(5) \\
& C(25)-\mathrm{Na}(1)-\mathrm{C}(27)-\mathrm{C}(26) \\
& -30.9(4) \\
& \mathrm{N}(4)-\mathrm{Na}(1)-\mathrm{C}(27)-\mathrm{C}(22) \\
& -113.5(5) \\
& O(1)-\mathrm{Na}(1)-\mathrm{C}(27)-\mathrm{C}(22) \\
& 126.9(5) \\
& \mathrm{C}(6) \# 1-\mathrm{Na}(1)-\mathrm{C}(27)-\mathrm{C}(22) \\
& -27.2(6) \\
& \mathrm{C}(5) \# 1-\mathrm{Na}(1)-\mathrm{C}(27)-\mathrm{C}(22) \\
& 5.2(6) \\
& C(26)-\mathrm{Na}(1)-\mathrm{C}(27)-\mathrm{C}(22) \\
& 126.3(7) \\
& \mathrm{C}(23)-\mathrm{Na}(1)-\mathrm{C}(27)-\mathrm{C}(22) \\
& 30.9(4) \\
& \mathrm{C}(24)-\mathrm{Na}(1)-\mathrm{C}(27)-\mathrm{C}(22) \\
& 63 . \odot(5) \\
& C(25)-\mathrm{Na}(1)-\mathrm{C}(27)-\mathrm{C}(22) \\
& 95.3(5) \\
& \mathrm{N}(4)-\mathrm{Na}(1)-\mathrm{C}(27)-\mathrm{N}(3) \\
& 4 . \odot(5) \\
& 0(1)-\mathrm{Na}(1)-\mathrm{C}(27)-\mathrm{N}(3) \\
& \mathrm{C}(6) \# 1-\mathrm{Na}(1)-\mathrm{C}(27)-\mathrm{N}(3) \\
& -115.5(6) \\
& 90.3(6) \\
& \mathrm{C}(5) \# 1-\mathrm{Na}(1)-\mathrm{C}(27)-\mathrm{N}(3) \\
& 122.7(5) \\
& \mathrm{C}(22)-\mathrm{Na}(1)-\mathrm{C}(27)-\mathrm{N}(3) \\
& 117.6(8) \\
& \mathrm{C}(26)-\mathrm{Na}(1)-\mathrm{C}(27)-\mathrm{N}(3) \\
& -116.2(8) \\
& \mathrm{C}(23)-\mathrm{Na}(1)-\mathrm{C}(27)-\mathrm{N}(3) \\
& 148.4(7) \\
& \mathrm{C}(24)-\mathrm{Na}(1)-\mathrm{C}(27)-\mathrm{N}(3) \\
& -179.4(7) \\
& \mathrm{C}(25)-\mathrm{Na}(1)-\mathrm{C}(27)-\mathrm{N}(3) \\
& -147.1(7) \\
& C(23)-C(22)-C(28)-C(29) \\
& 69.4(11)
\end{aligned}
$$




$$
\begin{aligned}
& C(27)-C(22)-C(28)-C(29) \\
& -111.9(9) \\
& \mathrm{Na}(1)-\mathrm{C}(22)-\mathrm{C}(28)-\mathrm{C}(29) \\
& -22.9(10) \\
& C(23)-C(22)-C(28)-C(30) \\
& -56.5(11) \\
& C(27)-C(22)-C(28)-C(30) \\
& 122.2(9) \\
& \mathrm{Na}(1)-\mathrm{C}(22)-\mathrm{C}(28)-\mathrm{C}(30) \\
& -148.9(7) \\
& C(25)-C(26)-C(31)-C(32) \\
& -78 . \odot(9) \\
& C(27)-C(26)-C(31)-C(32) \\
& \text { 103.8(9) } \\
& \mathrm{Na}(1)-\mathrm{C}(26)-\mathrm{C}(31)-\mathrm{C}(32) \\
& \text { 15.1(9) } \\
& C(25)-C(26)-C(31)-C(33) \\
& 47.8(10) \\
& C(27)-C(26)-C(31)-C(33) \\
& -130.4(8) \\
& \mathrm{Na}(1)-\mathrm{C}(26)-\mathrm{C}(31)-\mathrm{C}(33) \\
& 140.8(6) \\
& \mathrm{N}(4)-\mathrm{Na}(1)-\mathrm{O}(1)-\mathrm{C}(37) \\
& 19.1(8) \\
& \mathrm{C}(6) \# 1-\mathrm{Na}(1)-0(1)-\mathrm{C}(37) \\
& -80.6(7) \\
& \mathrm{C}(5) \# 1-\mathrm{Na}(1)-0(1)-\mathrm{C}(37) \\
& \mathrm{C}(27)-\mathrm{Na}(1)-0(1)-\mathrm{C}(37) \\
& -108.3(7) \\
& 115.8(7) \\
& \mathrm{C}(22)-\mathrm{Na}(1)-\mathrm{O}(1)-\mathrm{C}(37) \\
& 148.3(7) \\
& \mathrm{C}(26)-\mathrm{Na}(1)-0(1)-\mathrm{C}(37) \\
& 116.1(7) \\
& \mathrm{C}(23)-\mathrm{Na}(1)-0(1)-\mathrm{C}(37) \\
& 170.8(6) \\
& \mathrm{C}(24)-\mathrm{Na}(1)-0(1)-\mathrm{C}(37) \\
& 163.5(7) \\
& \mathrm{C}(25)-\mathrm{Na}(1)-0(1)-\mathrm{C}(37) \\
& 138.6(7) \\
& N(4)-N a(1)-0(1)-C(34) \\
& -163.6(8) \\
& \mathrm{C}(6) \# 1-\mathrm{Na}(1)-0(1)-\mathrm{C}(34) \\
& 96.7(8) \\
& \mathrm{C}(5) \# 1-\mathrm{Na}(1)-\mathrm{O}(1)-\mathrm{C}(34) \\
& 69.0(8) \\
& \mathrm{C}(27)-\mathrm{Na}(1)-0(1)-\mathrm{C}(34) \\
& -66.9(9) \\
& \mathrm{C}(22)-\mathrm{Na}(1)-0(1)-\mathrm{C}(34) \\
& -34.4(10) \\
& \mathrm{C}(26)-\mathrm{Na}(1)-\mathrm{O}(1)-\mathrm{C}(34) \\
& -66.6(8) \\
& \mathrm{C}(23)-\mathrm{Na}(1)-\mathrm{O}(1)-\mathrm{C}(34) \\
& -11.9(9) \\
& \mathrm{C}(24)-\mathrm{Na}(1)-\mathrm{O}(1)-\mathrm{C}(34) \\
& -19.1(8)
\end{aligned}
$$




$$
\begin{aligned}
& \mathrm{C}(25)-\mathrm{Na}(1)-\mathrm{O}(1)-\mathrm{C}(34) \\
& \mathrm{C}(37)-\mathrm{O}(1)-\mathrm{C}(34)-\mathrm{C}(35) \\
& \mathrm{Na}(1)-\mathrm{O}(1)-\mathrm{C}(34)-\mathrm{C}(35) \\
& \mathrm{O}(1)-\mathrm{C}(34)-\mathrm{C}(35)-\mathrm{C}(36) \\
& \mathrm{C}(34)-\mathrm{C}(35)-\mathrm{C}(36)-\mathrm{C}(37) \\
& \mathrm{C}(34)-\mathrm{O}(1)-\mathrm{C}(37)-\mathrm{C}(36) \\
& \mathrm{Na}(1)-0(1)-\mathrm{C}(37)-\mathrm{C}(36) \\
& \mathrm{C}(35)-\mathrm{C}(36)-\mathrm{C}(37)-0(1)
\end{aligned}
$$

$$
\begin{gathered}
-44.1(8) \\
-37.2(11) \\
145.2(8) \\
28.6(14) \\
-10.4(17) \\
30.5(12) \\
-151.5(9) \\
-11.9(16)
\end{gathered}
$$

Symmetry transformations used to generate equivalent atoms:

$\# 1-x+1, y+1 / 2,-z+1 / 2 \quad \# 2-x+1, y-1 / 2,-z+1 / 2$ 